\title{
IDENTIFICATION AND FUNCTIONAL CHARACTERIZATION OF PROTIST SWI/SNF AND ISWI COMPLEXES
}

\author{
by \\ Alejandro Saettone Chipana \\ Bachiller en Ciencias Biólogicas \\ Universidad Nacional Mayor de San Marcos, Lima-Perú \\ October 2008 \\ A thesis presented to Ryerson University \\ in partial fulfillment of the requirements for the degree of \\ Master of Science \\ in the Program of Molecular Sciences
}

Toronto, Ontario, Canada, 2015

(C) Alejandro Saettone Chipana, 2015 


\section{AUTHOR'S DECLARATION FOR ELECTRONIC SUBMISSION OF A THESIS}

I hereby declare that I am the sole author of this thesis. This is a true copy of the thesis, including any required final revisions, as accepted by my examiners.

I authorize Ryerson University to lend this thesis to other institutions or individuals for the purpose of scholarly research.

I further authorize Ryerson University to reproduce this thesis by photocopying or by other means, in total or in part, at the request of other institutions or individuals for the purpose of scholarly research.

I understand that my thesis may be made electronically available to the public. 


\begin{abstract}
The thesis aims to identify and initiate functional characterization of the SWI/SNF and ISWI complexes in Tetrahymena thermophila. Through affinity purification of the conserved subunit Snf5 followed by mass spectrometry (AP-MS), I identified the first SWI/SNF complex in protists. One of the subunits I found is a small bromodomain containing protein named Ibd1. Through AP-MS of Ibd1 I found Ibd1 is versatile and interacts with several additional chromatin remodeling complexes. Bromodomains are known to have affinity for acetylated lysine residues within proteins such as histones. A peptide array experiment suggests that Ibd1 also has affinity for acetylated chromatin. Indirect immunofluorescence (IF) of Ibd1 hints at a role in transcription. My analysis of Tetrahymena Iswi1 shows expression during meiosis, vegetative growth and starvation. IF data shows its localization is consistent with Iswi1 function in mitosis/meiosis or maintenance of silent chromatin. AP-MS of ISW1 discovered several interacting proteins of unknown function.
\end{abstract}




\section{Table of Contents}

IDENTIFICATION AND FUNCTIONAL CHARACTERIZATION OF PROTIST SWI/SNF

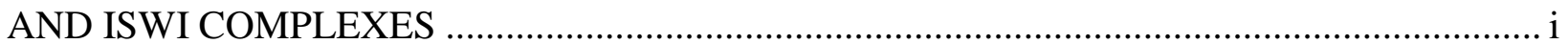

AUTHOR'S DECLARATION FOR ELECTRONIC SUBMISSION OF A THESIS ................... ii

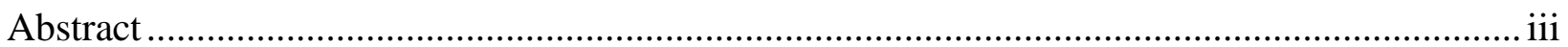

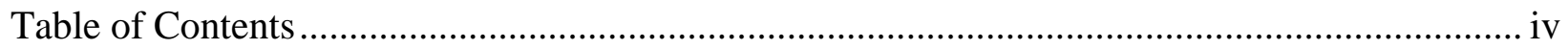

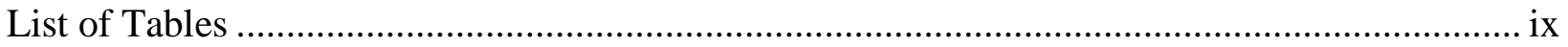

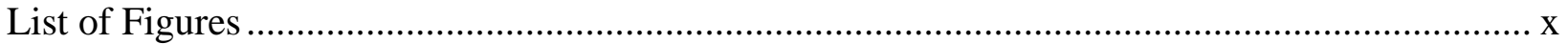

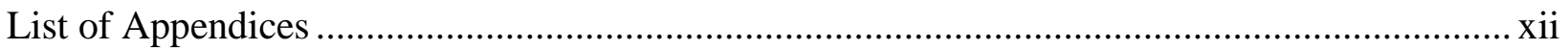

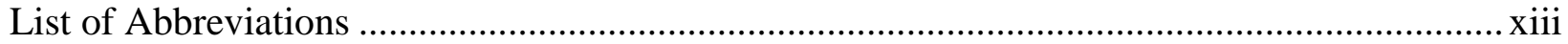

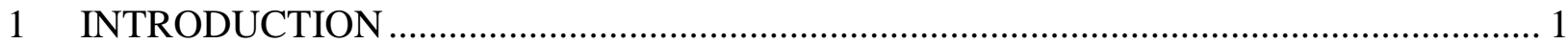

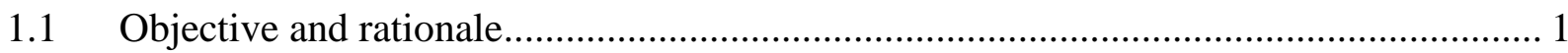

1.2 Tetrahymena thermophila as a model organism ..................................................... 2

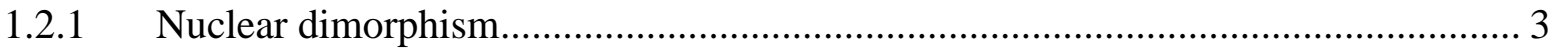

1.2.2 Tetrahymena life cycles ....................................................................... 3

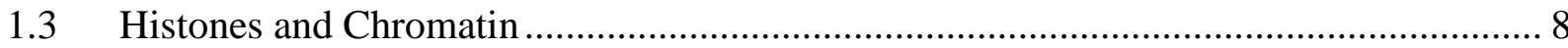

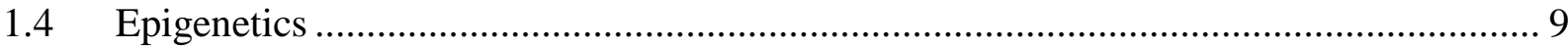

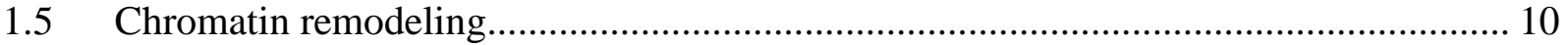

1.5.1 Post-translational modification (PTM) of histone proteins .............................. 10

1.5.2 ATP dependent chromatin remodeling complexes ....................................... 13

1.6 Starting point to identification of SWI/SNF in Tetrahymena .................................. 17

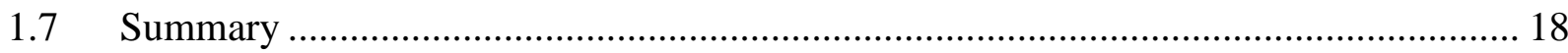

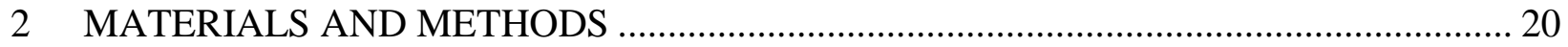


2.1 Strain generation - Construction of gene specific 3xFLAG-TEV-ZZ (FZZ) targeting

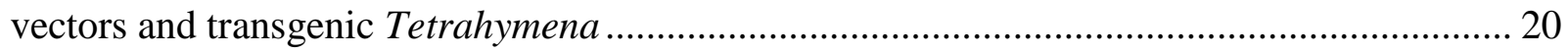

2.1.1 Tetrahymena thermophila cell strains, culture, cells collection, starvation and mating 22

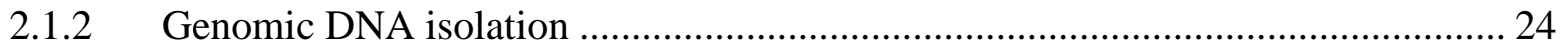

2.1.3 Sequence data retrieval and primers design .............................................. 25

2.1.4 PCR on genomic Tetrahymena DNA ..................................................... 26

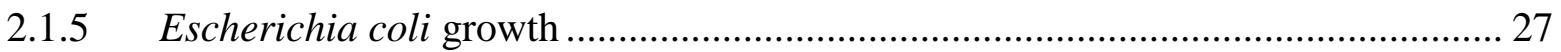

2.1.6 Plasmid isolation, pBKS-FZZ plasmid and PCR products restriction digestion, ligation, transformation and plasmid sequencing ................................................... 27

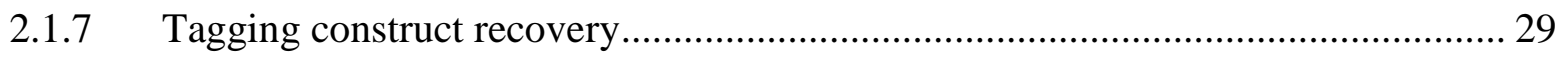

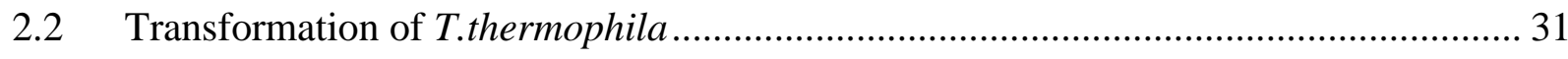

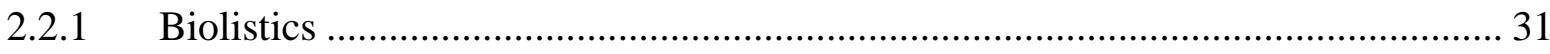

2.2.2 Cell passages and phenotypic assortment .............................................. 31

2.2.3 Trichloroacetic acid precipitation and Western blotting (TCA-WB) ................... 32

2.3 Affinity purification - Western blotting (AP-WB) ........................................... 34

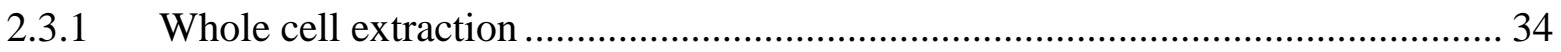

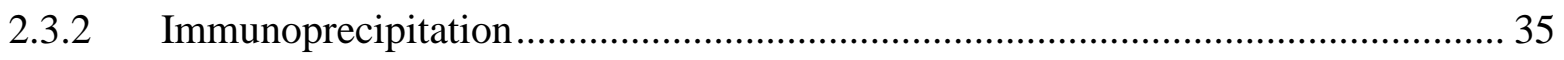

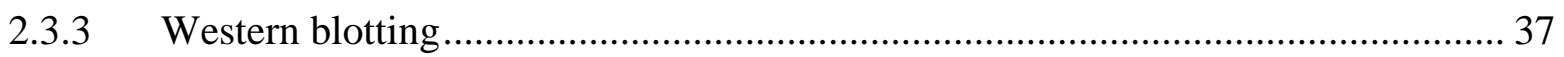

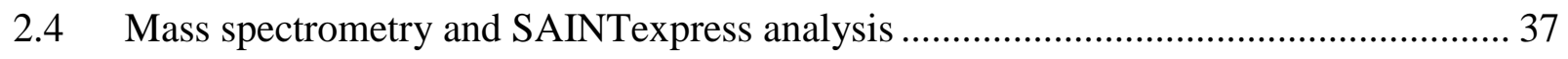

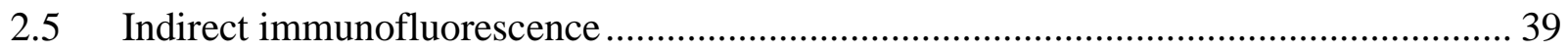

2.6 Comprehensive analysis of SWI/SNF complex .............................................. 40

2.7 Synthetic gene - cloning and expression of Tetrahymena thermophila Interactive

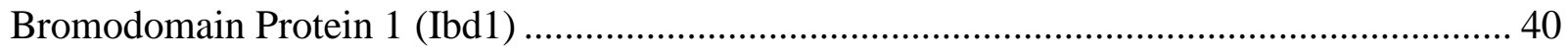

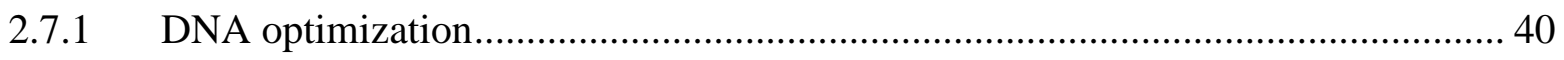

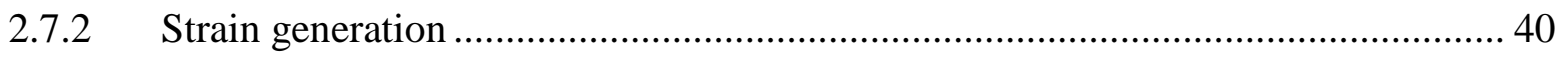


2.7.3 Inducing Expression of Ibd1 in E. coli BL21 (DE3) ..................................... 42

2.7.4 Purification of Expressed 6XHis-Ibd1 _.................................................... 42

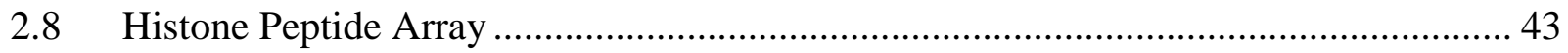

3 CHAPTER 1 - MOLECULAR CLONING OF TAGGING CONSTRUCTS AND GENERATION OF TAGGED LINES RESULTS .......................................................... 44

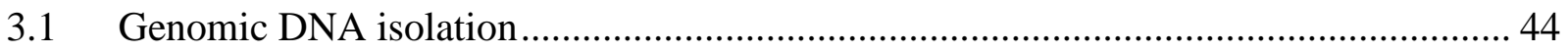

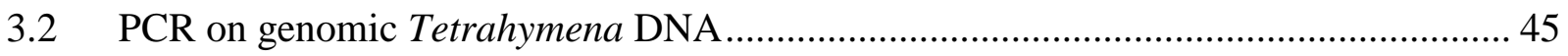

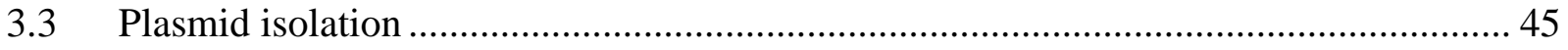

3.4 pBKS-FZZ plasmids and 1kbUP PCR products restriction digestion followed by

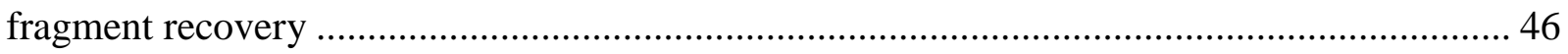

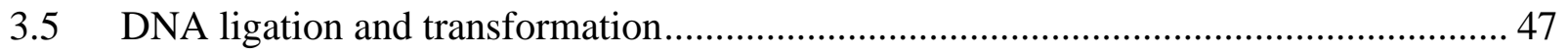

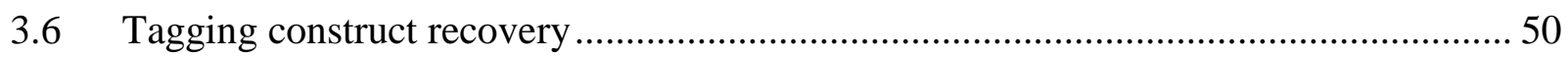

4 CHAPTER 2 - Identification of Tetrahymena SWI/SNF and identification and analysis of $\operatorname{Ibd} 152$

4.1 Assessment to determine if Snf5-FZZ pm-r transformants are expressing the tagged

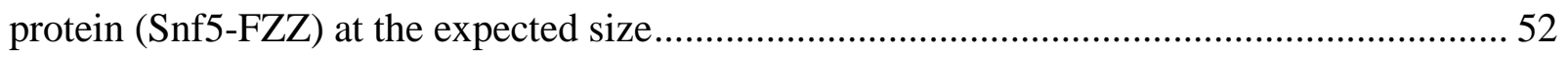

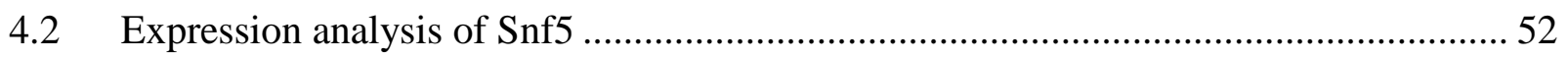

4.3 MS and SAINTexpress analysis of affinity purified Snf5-FZZ during vegetative growth 54

4.4 Molecular cloning and generation of FZZ tagged TTHERM_00729230 (Ibd1) .......... 56

4.5 Assessment to determine if pm-r transformants are expressing the tagged protein (BD-

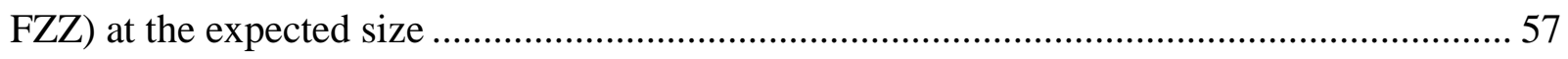

4.6 Analysis of Ibd1 interactions during vegetative growth .....................................5 57

4.7 SAINTexpress analysis of MS data from affinity purified BD-FZZ during vegetative

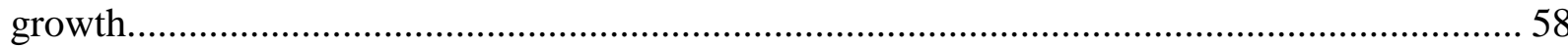

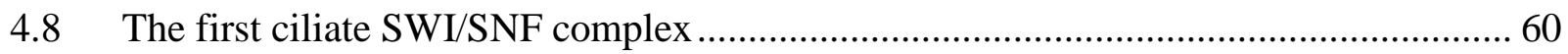

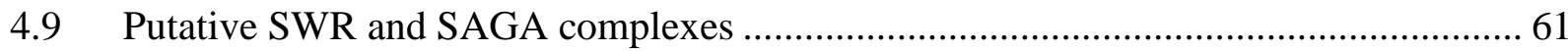


4.10 During vegetative growth Ibd1 interacts with members of chromatin remodeling

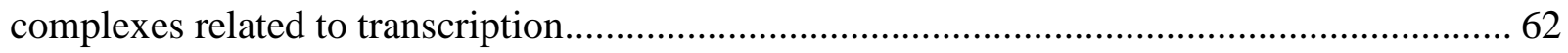

4.11 Expression analysis of BD-FZZ during T.thermophila's life cycle ............................ 63

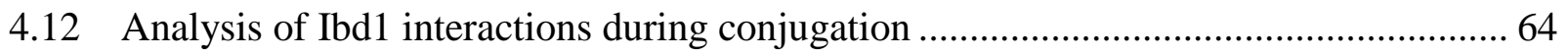

4.13 SAINTexpress curation of MS data from AP of Ibd1-FZZ during conjugation........... 65

4.14 Ibd1 modifies its interactions at 5 hours after conjugation and during vegetative growth 67

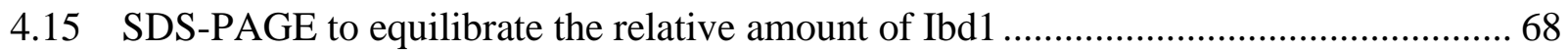

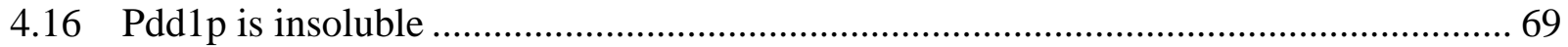

4.17 Ibd1 recognizes multiple rather than single PTM ............................................... 70

4.17.1 Strain generation ...................................................................................... 70

4.17.2 Inducing expression of Ibd1 in E. coli BL21 (DE3) ....................................... 71

4.17.3 Affinity Purification of 6XHis-Ibd1 from BL21 ........................................ 72

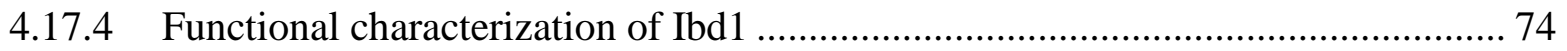

4.17.5 Peptide array for the control (His-G9a) ...................................................... 74

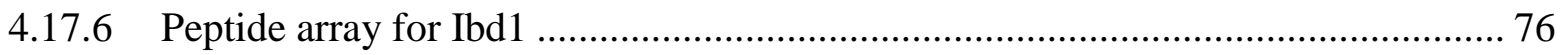

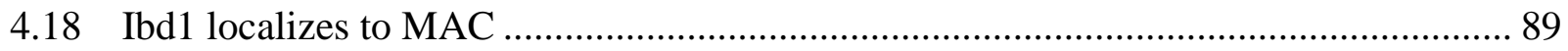

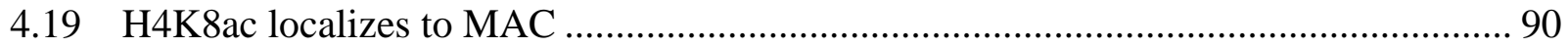

4.20 Model of Ibd1 localization during T.thermophila's life cycle ................................. 92

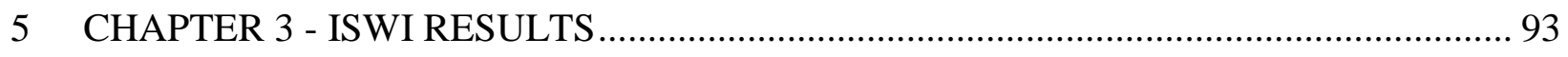

5.1 Characterization of potential ISW1-FZZ transformants during development ............. 94

5.1.1 Expression analysis to assess whether individual strains are expressing ISWI1-FZZ

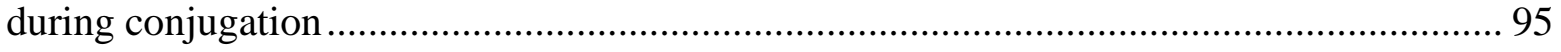

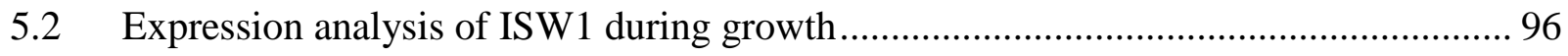

5.3 Expression analysis of ISWI1-FZZ during T.thermophila's life cycle....................... 97

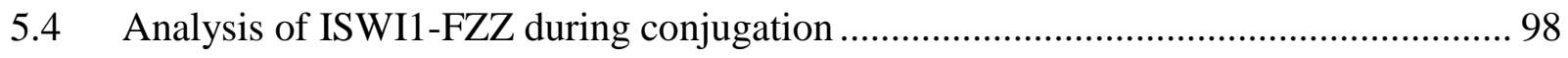


5.5 MS and SAINTexpress analysis of ISWI1-FZZ during conjugation 98

5.6 Iswi1 localizes to the MIC during growth and sexual development including meiosis100

5.7 Model of ISWI1 localization during T.thermophila's life cycle ............................. 102

6 SUMMARY AND FUTURE DIRECTIONS .......................................................... 103

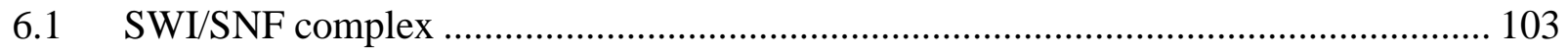

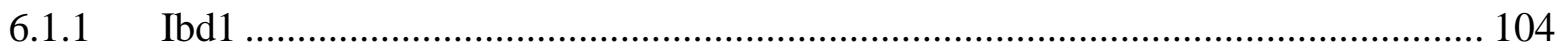

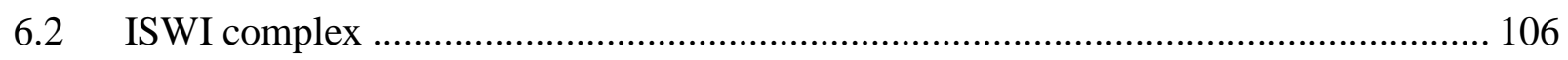

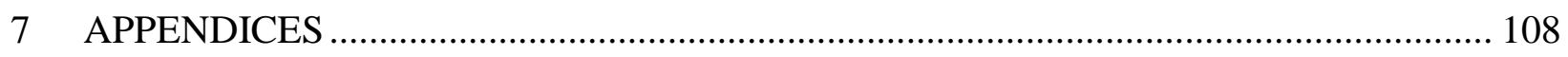

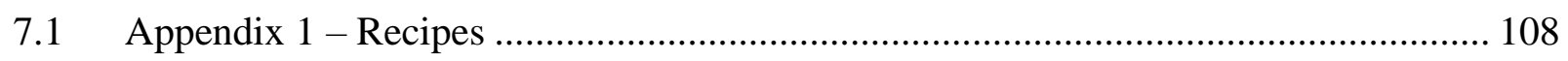

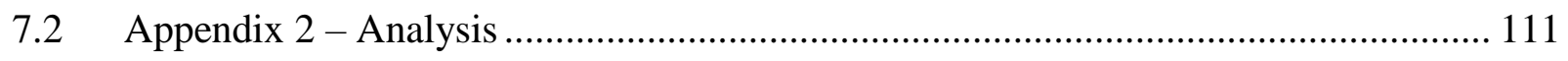

7.3 Appendix 3 - Primers................................................................................ 112

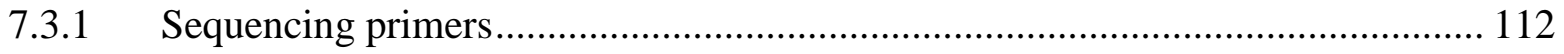

7.3.2 Bd containing protein (TTHERM_00729230) primers .................................... 112

7.3.3 ISWI1 (TTHERM_00388250) primers.................................................... 112

7.3.4 Synthetic Ibd1 gene primers .................................................................. 113

7.4 Appendix 4 - Protein ladder........................................................................ 114

7.5 Appendix 5 - Peptide Array .......................................................................... 115

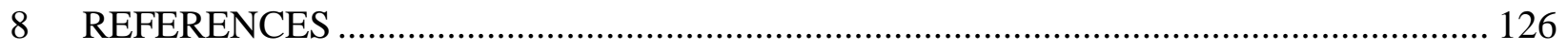




\section{List of Tables}

Table 1: Predicted subunit of the SWI/SNF complex in Tetrahymena .................................... 18

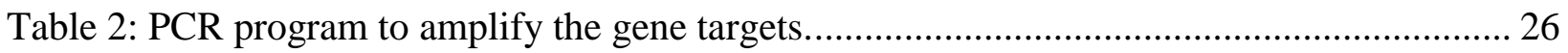

Table 3: Conditions for incubation of Antibodies .................................................................... 33

Table 4: SAINTexpress-curated MS data for 4 replicates of SNF5-FZZ affinity purification

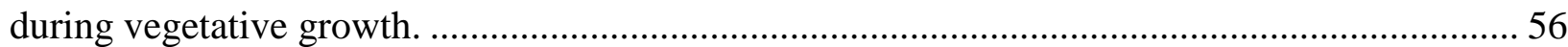

Table 5: SAINTexpress-curated data for 3 replicates of BD-FZZ during vegetative growth ...... 59

Table 6: Tetrahymena thermophila SWI/SNF complex ..................................................... 61

Table 7. Tetrahymena thermophila putative SWR complex ............................................... 61

Table 8. Tetrahymena thermophila putative SAGA complex .................................................. 62

Table 9: SAINTexpress-curated data for 2 replicates of BD-FZZ 5 hours after mating. ............ 66

Table 10: SAINTexpress-curated data for 2 replicates of ISWI1-FZZ during conjugation ......... 99

Table 11: Data analysis for Ibd1 during vegetative growth and 5 hours after conjugation........ 111 


\section{List of Figures}

Figure 1. Phylogenetic tree showing bacteria, archea and eukaryota. ....................................... 2

Figure 2: Acceleration of phenotypic assortment of alleles in T.thermophila ........................... 5

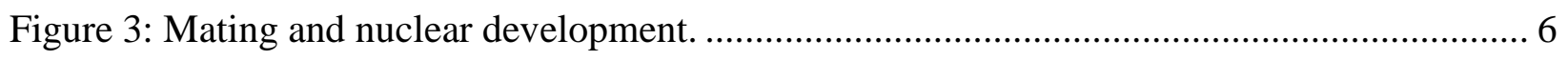

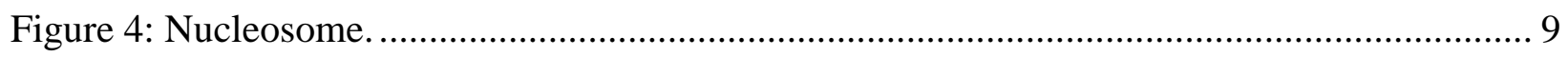

Figure 5: Human and Tetrahymena histone alignments .................................................... 12

Figure 6: Mechanisms of chromatin remodeling ................................................................ 14

Figure 7: Bromodomains physically interacts with acetylated histones................................. 17

Figure 8: pBKS-FZZ plasmid MAP. (Garg et al., 2013a) ................................................... 21

Figure 9: Two DNA isolations from whole cell genomic DNA............................................. 44

Figure 10: PCR on genomic Tetrahymena DNA for BD (TTHERM_00729230) and Iswi1

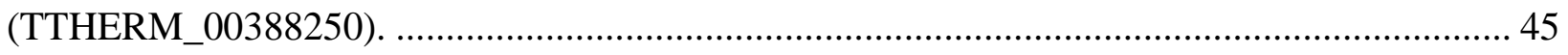

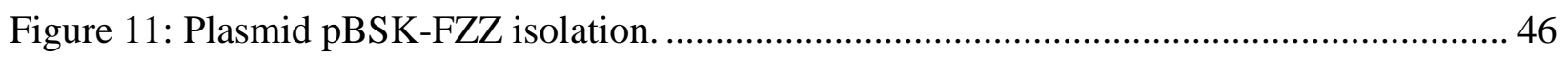

Figure 12: Restriction digestion with KpnI and XhoI ..................................................... 47

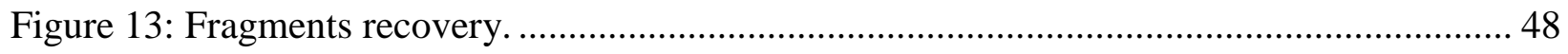

Figure 14: pBKS-FZZ Plasmids after 1kb UP transformation. ........................................... 48

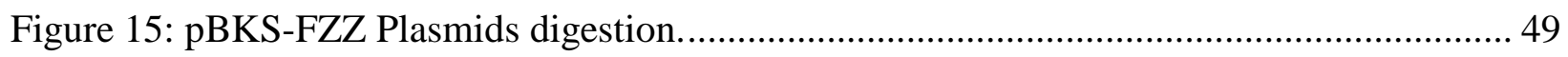

Figure 16: Double digestion to obtain the tagging constructs. ................................................ 50

Figure 17: Tagging construct clean up....................................................................... 51

Figure 18. Expression Analysis / Affinity Purification of SNF5-FZZ ................................... 54

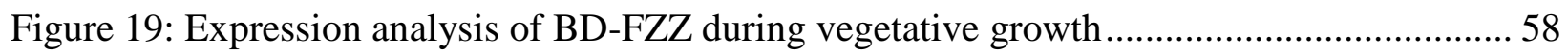

Figure 20: Comprehensive analysis of the Ibd1 interactome during vegetative growth. ............ 63

Figure 21: Expression analysis of Ibd1-FZZ during T.thermophila's life cycle ........................ 64

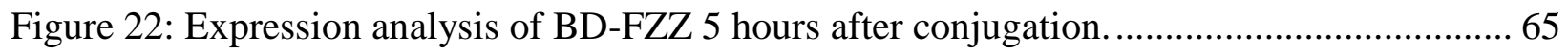

Figure 23: Relative proportion of encountered proteins during vegetative growth and 5 hours

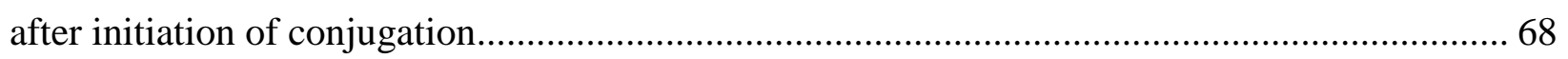

Figure 24: SDS-PAGE to equilibrate the relative amount of Ibd1 and Pdd1p solubility............ 69

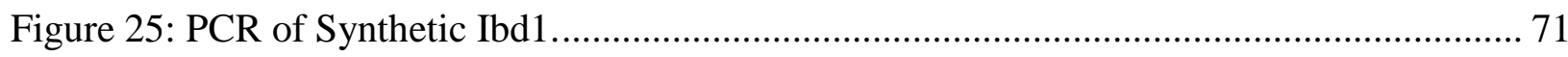

Figure 26: Inducing expression of Ibd1 in E.coli BL21 (DE3). ............................................ 72

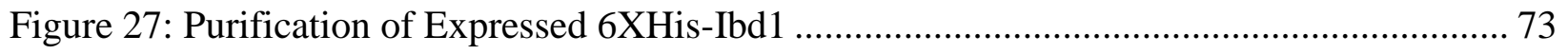


Figure 28: Buffer exchange by size exclusion chromatography ........................................... 73

Figure 29. Raw data of the peptide array for the control (His-G9a)....................................... 75

Figure 30: Multiple peptides specificity analysis for the control (His-G9a) ........................... 76

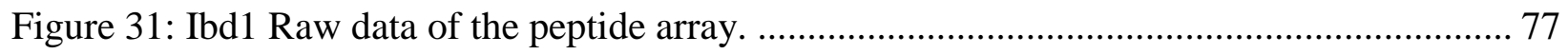

Figure 32: Multiple peptides specificity analysis for Ibd1 ............................................... 78

Figure 33: Single peptide reactivity analysis for hH4K8ac............................................... 79

Figure 34: Single peptide reactivity analysis for hH2AK13ac. ........................................... 80

Figure 35: Single peptide reactivity analysis for hH4K12ac.......................................... 81

Figure 36: Single peptide reactivity analysis for hH4K5ac.............................................. 82

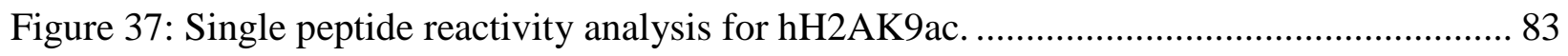

Figure 38: Single peptide reactivity analysis for H2AK5 ................................................. 84

Figure 39: Single peptide reactivity analysis for H4K20ac............................................... 85

Figure 40: Single peptide reactivity analysis for H3K36ac............................................. 86

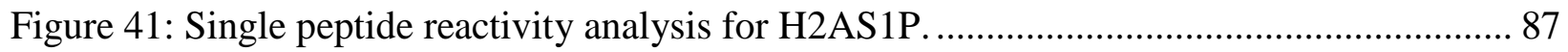

Figure 42: Single peptide reactivity analysis for hH4K16ac.............................................. 88

Figure 43: Ibd1 and H4K8Ac localizes to the MAC during growth and sexual development

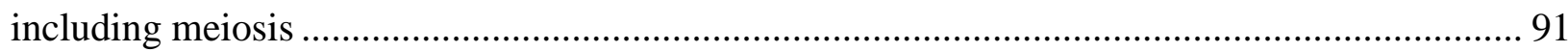

Figure 44: Model of Ibd1 localization during T.thermophila's life cycle. .............................. 92

Figure 45: Iswi1 gene expression profile from RNA-seq ................................................ 93

Figure 46: Intermediated molecules involved in T.thermophila's DNA rearrangement............. 94

Figure 47: Characterization of potential ISW1-FZZ transformants. .................................... 95

Figure 48: Expression analysis to assess whether individual strains are expressing ISWI1-FZZ

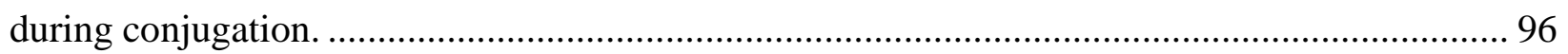

Figure 49: Expression analysis of ISWI1-FZZ during vegetative growth.............................. 97

Figure 50: Expression analysis of ISWI1-FZZ during T.thermophila's life cycle ..................... 97

Figure 51: Expression analysis of ISWI1-FZZ during conjugation....................................... 98

Figure 52: TTHERM_00247110 gene expression profile from RNA-seq ................................ 99

Figure 53: Iswi1 localizes to the MIC during growth and sexual development including meiosis 101

Figure 54: Model of ISWI1 localization during T.thermophila's life cycle ........................... 102 


\section{List of Appendices}

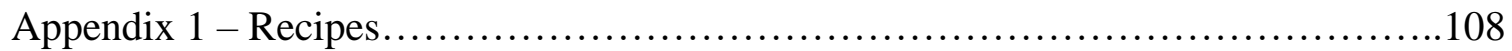

Appendix 2 - Analysis.......................................................... 111

Appendix 3 - Primers........................................................... 112

Appendix 4 - Protein ladder........................................................... 114

Appendix 5 - Peptide Array........................................................115 


\section{List of Abbreviations}

\begin{tabular}{|c|c|}
\hline${ }^{\circ} \mathrm{C}$ & degrees Celsius \\
\hline$\mu$ & micro \\
\hline $\mathrm{AC}$ & acetylation \\
\hline ADP & adenosine diphosphate \\
\hline AP & affinity purification \\
\hline APS & ammonium persulfate \\
\hline ATP & adenosine triphosphate \\
\hline $\mathrm{b}$ & base \\
\hline $\mathrm{BD}$ & bromodomain \\
\hline BLAST & Basic Local Alignment Search Tool \\
\hline BRCA1 & breast cancer type 1 susceptibility protein \\
\hline C- & carboxy \\
\hline $\mathrm{Da}$ & Dalton \\
\hline DAPI & 4',6-diamidino-2-phenylindole \\
\hline DCL1 & dicer-like protein 1 \\
\hline $\mathrm{ddH} 2 \mathrm{O}$ & double distilled water \\
\hline DOWN & downstream \\
\hline DNA & deoxyribonucleic acid \\
\hline dNTP & deoxynucleotides \\
\hline ECL & enhanced chemiluminescence \\
\hline EDTA & ethylenediaminetetraacetic acid \\
\hline FITC & fluorescein isothiocyanate \\
\hline FZZ & 3x FLAG-ZZ epitope tag \\
\hline $\mathrm{g}$ & grams \\
\hline $\mathrm{H}$ & hours \\
\hline $\mathrm{H} 2 \mathrm{~A}$ & histone $2 \mathrm{~A}$ \\
\hline $\mathrm{H} 2 \mathrm{Az}$ & histone $2 \mathrm{~A}$ variant $\mathrm{z}$ \\
\hline $\mathrm{H} 2 \mathrm{~B}$ & histone $2 \mathrm{~B}$ \\
\hline $\mathrm{H} 3$ & histone 3 \\
\hline
\end{tabular}




$\begin{array}{ll}\text { H4 } & \text { histone } 4 \\ \text { HAT } & \text { histone acetyltransferase } \\ \text { HMT } & \text { histone methyltransferase } \\ \text { HPLC } & \text { high pressure liquid chromatography } \\ \text { HRP } & \text { horseradish peroxidase } \\ \text { Ibd1 } & \text { Interactive Bromodomain Protein 1 } \\ \text { Ibd1-FZZ } & \text { refers to the FZZ strain } \\ \text { Ibd1-FZZ } & \text { refers to the tagged Ibd1 } \\ \text { IES } & \text { internal eliminated sequence } \\ \text { IF } & \text { indirect immunofluorescence } \\ \text { IgG } & \text { immunoglobulin G } \\ \text { INI1 } & \text { integrase interactor 1 } \\ \text { IP } & \text { immunoprecipitation } \\ \text { ISWI } & \text { Imitation SWI } \\ \text { ISWI1-FZZ } & \text { refers to the FZZ strains } \\ \text { Iswi1-FZZ } & \text { refers to the tagged Iswi1 } \\ \text { Iswi1 } & \text { Imitation SWI 1 protein } \\ \text { Iswi 2 } & \text { Imitation SWI 2 protein } \\ \text { k } & \text { kilo } \\ \text { KO } & \text { knock-out } \\ \text { L } & \text { litres } \\ \text { LC } & \text { liquid chromatography } \\ \text { Log } & \text { logarithmic } \\ \text { LTQ } & \text { Linear Trap Quadrupole } \\ \text { m } & \text { milli } \\ \text { M } & \text { molar } \\ \text { MAC } & \text { macronucleus } \\ \text { MIC } & \text { micronucleus } \\ \text { MS } & \text { mass spectrometry } \\ \text { MS-AP } & \text { mass spectrometry- affinity purification } \\ \text { mt } & \text { mating type } \\ & \end{array}$




$\begin{array}{ll}\text { N } & \text { normal } \\ \text { N- } & \text { amino } \\ \text { n } & \text { haploid number } \\ \text { ncRNA } & \text { non-coding RNA } \\ \text { NURF } & \text { nucleosome remodeling factor } \\ \text { Ori } & \text { origin of replication } \\ \text { PCR } & \text { polymerase chain reaction } \\ \text { p } & \text { pico } \\ \text { pH } & \text { potential hydrogen } \\ \text { pm-r } & \text { paramomycin resistant } \\ \text { PTM } & \text { posttranslational modification } \\ \text { rDNA } & \text { ribosomal deoxyribonucleic acid } \\ \text { Rho } & \text { rhodamine } \\ \text { RNA } & \text { ribonucleic acid } \\ \text { RP-HPLC } & \text { reversed-phase high-pressure liquid chromatography } \\ \text { rpm } & \text { rotations per minute } \\ \text { RSC } & \text { remodel the structure of chromatin } \\ \text { SAGA } & \text { multi-subunit histone modifying complex } \\ \text { SAINT } & \text { significance analysis of interactome } \\ \text { scnRNA } & \text { scan RNA } \\ \text { SDS-PAGE } & \text { sodium dodecyl sulfate polyacrylamide gel electrophoresis } \\ \text { SNF5-FZZ } & \text { refers to the FZZ strains } \\ \text { Snf5-FZZ } & \text { refers to the tagged protein } \\ \text { SPP } & \text { sequestering protease peptone } \\ \text { SWI/SNF } & \text { SWItching/Sucrose Non-Fermenting } \\ \text { SWR } & \text { histone variant exchange complex } \\ \text { TCA } & \text { trichloroacetic acid } \\ \text { TEV } & \text { tobacco edge virus } \\ \text { TGD wiki } & \text { Tetrahymena genome database wiki } \\ \text { TF } & \text { transcription factor } \\ \text { Tt } & \text { Tetrahymena thermophila } \\ & \end{array}$




$\begin{array}{ll}\text { U } & \text { unit } \\ \text { UP } & \text { upstream } \\ \text { UTR } & \text { untranslated region } \\ \text { UV } & \text { ultraviolet } \\ \text { V } & \text { volts } \\ \text { Veg } & \text { vegetative growth } \\ \text { WB } & \text { Western Blot } \\ \text { WCE } & \text { whole cell extract } \\ \text { WT } & \text { wild type } \\ \text { w/v } & \text { weight/ volume }\end{array}$




\section{INTRODUCTION}

\subsection{Objective and rationale}

In multi-cellular eukaryotes, the precise function of ATP-dependent chromatin remodeling complexes such as the SWI/SNF (SWItch/Sucrose Non Fermentable) and the ISWI (Imitation SWI) complexes, remains unclear. In humans, alteration or loss of the SWI/SNF complex function through mutation has been shown to be associated with cancer and there is evidence that relates SWI/SNF and ISWI complexes to tumor suppression (Pal, Vishwanath, Tempst, Sif, \& Erdjument-bromage, 2004)' (Wong et al., 2000)' (Bochar et al., 2000) (Badenhorst, Voas, Rebay, $\& \mathrm{Wu}, 2002)$. In addition, SWI/SNF miss regulation critically affects cellular differentiation and proliferation (Reisman, Glaros, \& Thompson, 2009)' (Roberts \& Orkin, 2004). In Drosophila, the ISWI ( also called NURF complex), is required for proper binding of heat-shock transcription factors to their target genes (Badenhorst et al., 2002).

The objective of this research is characterize the composition as well as molecular function and mechanism of the SWI/SNF and ISWI complexes in the unique chromatin environment of a protist model, the Alveolate Tetrahymena thermophila.

This research will further identify molecular functions of SWI/SNF and ISW1 and possibly molecular mechanisms underlying ATP-dependent chromatin remodeling which is a vital process in eukaryotic cells. By analogy, it will also aide in our current understanding of how $S W I / S N F$ miss-regulation or altered function is involved in disease. In addition, T.thermophila is the most experimentally amenable member of the Alveolates and thus can help us to understand the basic biology of the parasitic and disease-causing Alveolate Plasmodium species. Tetrahymena thermophila is a proven model in establishing knowledge of relevance as it relates to the improvement of human health. The fundamental knowledge generated from this research will contribute to a greater understanding of human diseases, which include both chronic; such as cancer, and infectious; such as malaria. 


\subsection{Tetrahymena thermophila as a model organism}

Tetrahymena thermophila is the model system for this research. Tetrahymena thermophila is part of the superphylum of Alveolates within protists. Protists are eukaryotic unicellular animals and plants. Alveolates has three primary phyla (Figure 1): Ciliates, Dinoflagellates, and the parasitic phylum Apicomplexa. The Apicomplexa phylum contains the parasite Plasmodium falciparum, which is responsible for malaria. The ciliate phylum contains parasites such as Ichthyophthirius multifiliis that affects aquaculture (Sigh, Lindenstrøm, \& Buchmann, 2004). The ciliate T.thermophila is the most experimentally amenable member of the Alveolates and of the diverse ciliate species that include free-living organisms. Studies in T.thermophila have led directly to two Nobel Prizes: description of catalytic self-splicing RNA in the group I intron of the rDNA gene (Cech, 1990), and cloning of telomeres and identification of telomerase (Blackburn, Greider, \& Szostak, 2006). Studies in Tetrahymena also led to the identification of the first histone acetyltransferase (HAT) which established the link between histone acetylation and transcription (Brownell et al., 1996). Currently Tetrahymena is a leading model for studies of the links between RNAi and chromain formation (Mochizuki, 2012).

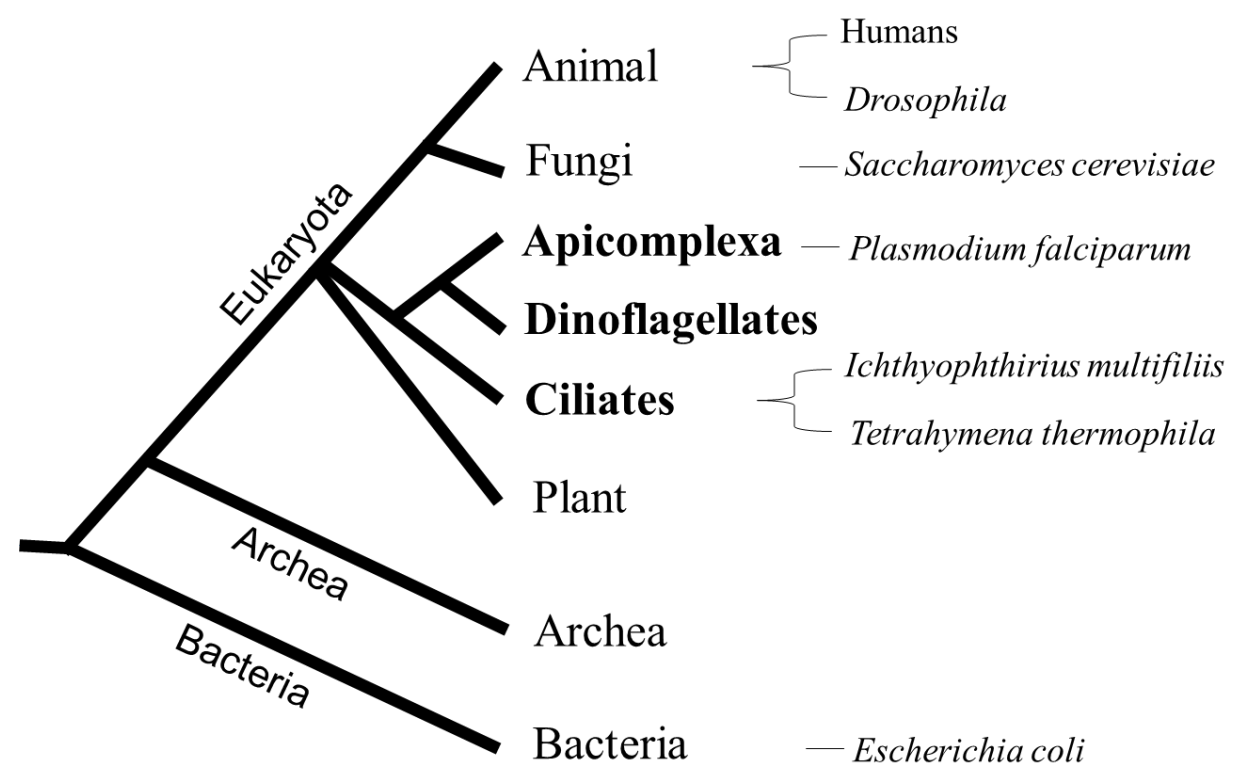

Figure 1. Phylogenetic tree showing bacteria, archea and eukaryota.

The protists branch shows the 3 phyla in bold (ciliate, dinoflagellates and apicomplexa). The phylum ciliate shows T.thermophila and the parasite I.multifiliis and plasmodium. The phylum apicomplexa shows P.falciparum.

Adaptation from (Nash, Nisbet, Barbrook, \& Howe, 2008)(Roy \& Morse, 2013) 


\subsubsection{Nuclear dimorphism}

The utility of T.thermophila as a model system for nuclear structure/function and chromatin studies lies in its nuclear dimorphism, with two distinct nuclei in its single eukaryotic cell. The first of these two nuclei has an amitotic and polyploid $(\mathrm{n}=250)$ macronucleus (MAC). Amitosis involves nuclear division without nuclear envelope degradation, chromosome condensation, establishment of clear mitotic spindles and equal chromosome segregation (Wolfe, 1967). Therefore the segregation of alleles to daughter cells during cell division is random (Merriam \& Bruns, 1988). The second is a mitotic and diploid ( $\mathrm{n}=5)$ micronucleus (MIC) (K. Collins, 2012). Gene expression from the MAC is responsible for vegetative growth and mating while the MIC is transcriptionally silent during vegetative growth (K. Collins, 2012) but is transcribed early in conjugation in meiosis (Martindale, Allis, \& Bruns, 1985). Much of the utility of T.thermophila to basic biomedical research has its origins in investigations of the fundamental differences between the two nuclei (Cech, 1990)(Brownell et al., 1996)(Mochizuki, Fine, Fujisawa, \& Gorovsky, 2002) (Blackburn et al., 2006).

\subsubsection{Tetrahymena life cycles}

\subsubsection{Vegetative growth}

Under laboratory conditions the optimal vegetative growth of T.thermophila is induced at $30^{\circ} \mathrm{C}$ and slow shaking (100rpm). During vegetative growth T.thermophila cells reproduce by binary fission (Orias, Cervantes, \& Hamilton, 2011). The MIC undergoes meiosis and the MAC undergoes amitosis (K. Collins, 2012). Although during conjugation MAC develops from the diploid MIC, for any gene that is heterozygous in the MIC the new MAC will initially contain equal amount of both alleles but the following a number of amitotic divisions will lead to unequal ratios of the alleles in daughter cells. Gene expression from the MAC provides the cell's phenotype and its amitosis during vegetative growth can produce different phenotypes among the progeny. At some point the cells can lose all copies of one of the alleles for a particular gene as long as the lost allele is non-essential for viability. This phenotypic appearance of a homozygous 
trait in the MAC from an initially heterozygous situation is called phenotypic assortment (Merriam \& Bruns, 1988).

As mentioned above, Tetrahymena thermophila vegetative growth is based on asexual reproduction by binary fission (Orias et al., 2011). The MAC undergoes amitosis, therefore chromosomes, and as a result alleles, are not equally segregated to each daughter cell leading to phenotypic assortment. Tetrahymena MAC amitotic division naturally leads to phenotypic assortment. Phenotypic assortment can be accelerated by inserting selectable markers into T.thermophila's MAC genome, such as the neo cassette, using genetic transformation methods. The neo cassette contains a point mutation in the 17S rRNA gene that confers resistance to the antibiotic paromomycin (Yu, Hasson, \& Blackburn, 1988). Therefore, acceleration of phenotypic assortment is possible by gradually increasing the concentration of paromomycin into the growth media (Figure 2). During the binary fissions of phenotypic assortment, the MIC undergoes conventional mitosis and therefore equal numbers of chromosomes are portioned to each daughter cell and therefore MIC chromosomes are not subject to phenotypic assortment phenomenon (Orias et al., 2011). 


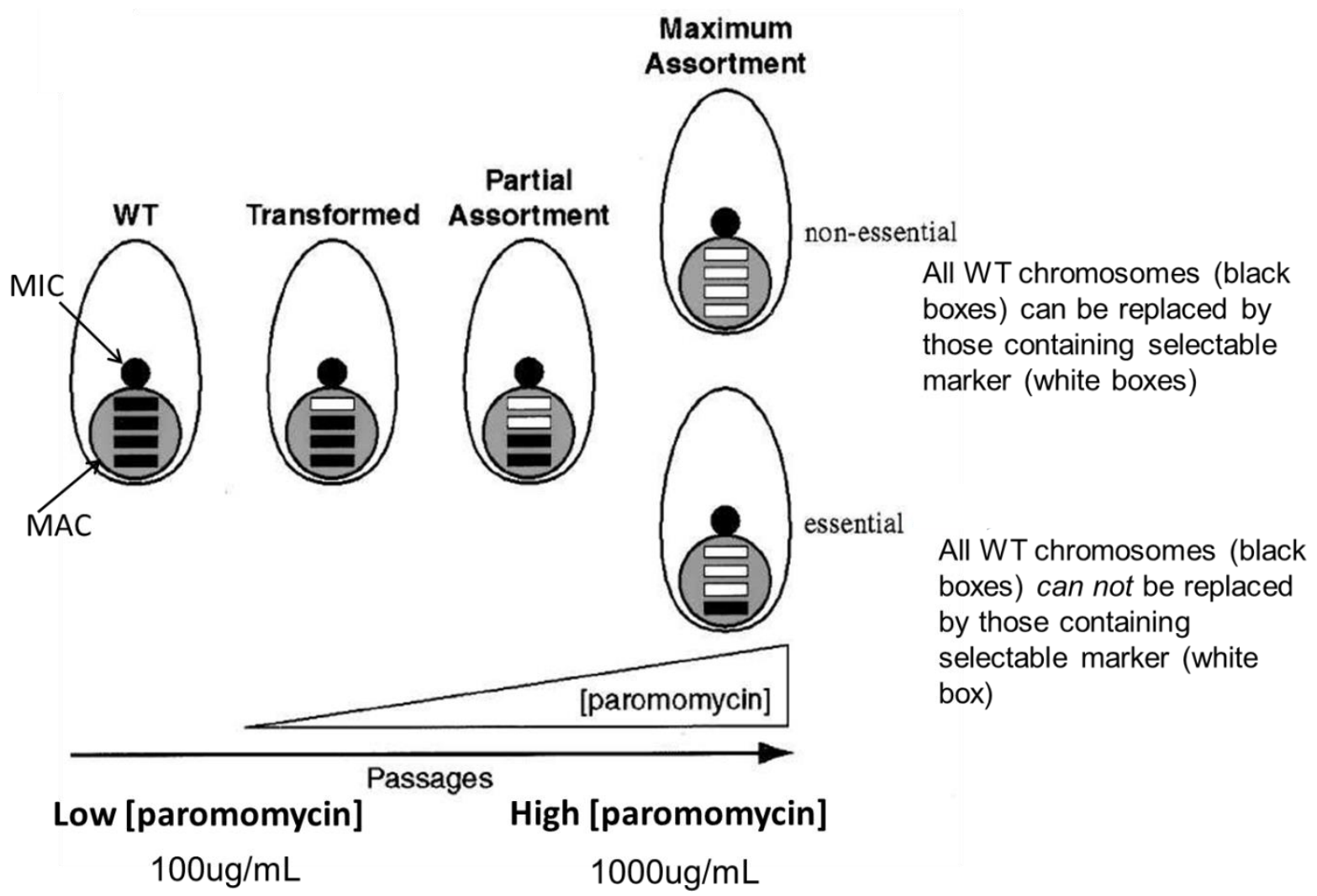

Figure 2: Acceleration of phenotypic assortment of alleles in T.thermophila. (Adapted from Miller and Collins, 2000)

In this figure the two cases of maximum assortment are differentiated by whether or not the transgenic MAC chromosome (white box) has some kind of deleterious effect (for example, if the selectable marker is being used to disrupt a gene you get the full replacement if the gene is non-essential for growth - partial replacement if essential for growth

\subsubsection{Conjugation}

Under laboratory conditions mating of T.thermophila is induced by starvation. During starvation there is expression of two important genes (MTA and $M T B$ ) that encode for mating type-specific segments and a transmembrane protein. These proteins are essential for starved cells of different mating types to carry out with attachment and conjugation (Cervantes et al., 2013). The first step is cell pairing (Figure 3 a). Meiosis initiates and the MIC becomes transcriptionally active (Martindale, Allis, \& Bruns, 1982) for the only time in the cell's life cycle. As part of meiosis, the MIC elongates forming the crescent (prophase I) (Figure $3 \mathrm{~b}$ ). The end result of meiosis is four haploid gametic nuclei (Figure $3 \mathrm{c}$ ), one of which will duplicate mitotically to form two active and identical gametic nuclei (Figure $3 \mathrm{~d}$ ). The next step involves 
reciprocal exchange of one of the gametic nuclei of each cell then fusion of two different gametic nuclei to form diploid zygotic nuclei (Figure $3 \mathrm{e}$ ). At this point the zygotic nucleus is present and the degradation of the 3 inactive gametic nuclei occurs (Figure $3 \mathrm{f}$ ). A double mitotic duplication of the zygotic nuclei occurs forming four identical nuclei occurs and degradation of parental MAC (OLD MAC) starts (Figure $3 \mathrm{~g}$ ). Of the four new post-zygotic nuclei, two will develop to form two new MACs (anlagen), one will be degraded and one will mitotically duplicate to form two new MIC (Malone, Anderson, Motl, Rexer, \& Chalker, 2005). (Figure 3 h). MAC development occurs in both the MACs in the cell and involves programmed DNA rearrangements which include DNA fragmentation and DNA deletion losing 10-15\% from the original zygotic (MIC) genome (K. Collins, 2012). After a cell division with no associated nuclear division, the result of conjugation is four daughter cells with two newly formed nuclei that will have different genomes and different chromatin structures (K. Collins, 2012). (Figure 3 i). In conclusion, Tetrahymena thermophila mating, or conjugation, involves MAC development which includes large scale genome rearrangements that are regulated in large part at the level of chromatin structure (Smothers, Mizzen, Tubbert, Cook, \& Allis, 1997). Interestingly, these DNA rearrangements are controlled at some level by the cell's RNAi machinery (Mochizuki et al., 2002).

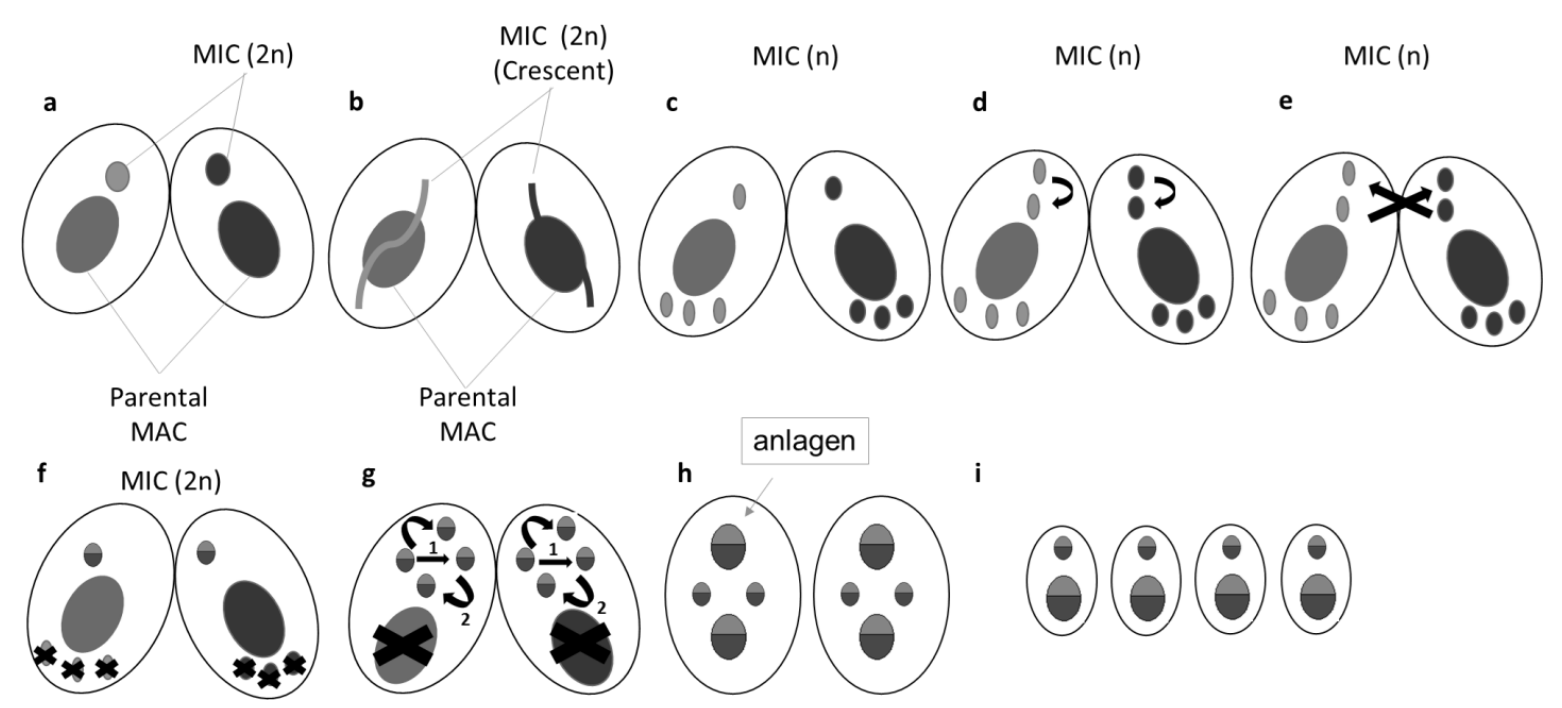

Figure 3: Mating and nuclear development.

a. Two starved cells of two different mating types attached; b. meiosis begins following formation of the crescent (prophase I); c. the result of meiosis is four gametic nuclei; d. one of which will duplicate continue to form two identical haploid gametic nuclei in each of the two cells; reciprocal exchange and fusion of the two active gametic nuclei; $f$. formation of the zygotic nucleus and degradation of the 3 inactive gametic nuclei; g. double duplication of the post- 
zygotic nuclei and degradation of OLD MAC starts; h. swelling of two zygotic nuclei to form anlagen which will become the new MAC; i. the result of conjugation is four daughter cells.

\subsection{DNA rearrangements in Tetrahymena thermophila}

Post-transcriptional gene silencing (PTGS) is an epigenetic mechanism that, as its name implies, is related to gene silencing (Mochizuki \& Gorovsky, 2004b). PAZ and Piwi containing domain proteins (PPD), such as the argonautes AGO1 and AGO2, are involved in PTGS through the formation of complexes with small functional RNAs (sRNA) that after transcription were initially long and were cut by a Dicer protein (DCL1 in Tetrahymena (Malone et al., 2005)). It is important to note that in Tetrahymena these sRNA are called scnRNA. At this point the protein or complex(es) that regulates the expression of the scnRNA precursor or long RNA is unknown; however, it is known they originate from the meiotic MIC and that RNAPII transcribes them (Mochizuki \& Gorovsky, 2004a). Dicer proteins were first described in the botanical model Arabidopsis thaliana as the catalytic subunit of the RNA-induced silencing complex (RISC). RISC directs gene silencing by RNA interference (RNAi) (Slotkin \& Martienssen, 2007). AGO1 and AGO2 bind many classes of sRNA, such as micro RNAs (miRNA), small interfering RNAs (siRNAs) and Piwi-interacting RNAs (piRNAs) (Slotkin \& Martienssen, 2007). Another example of PPD proteins are the Piwi-related proteins, which are nucleic acid-binding proteins that bind and cleave RNA, similar to the above described argonautes. One of Piwi-related proteins function (Twip1 in T.thermophila (Mochizuki et al., 2002)) is gene silencing (Slotkin \& Martienssen, 2007) via heterochromatin formation. In Tetrahymena, the heterochromatin is eliminated during IES deletion as part of the large scale DNA rearrangements that occur during MAC development in conjugation (Mochizuki et al., 2002).

During heterochromatin formation and subsequent elimination, the ultimate goal of the RISC-like complex Dicer/sRNA/PPD complex (in T.thermophila: DCL1/scnRNA/Twi1p) is to recruit histone lysine methyltransferases (HMT; example in T.thermophila: Ezl1p) so this HMT is able to methylate the target histone indicated by the sRNA. During T.thermophila's conjugation, RNAi-mediated heterochromatin formation and elimination starts with the transcription from the meiotic MIC of relatively long unprocessed RNA (Chalker \& Yao, 2001). These unprocessed RNA are cut in the cytoplasm by DCL1 into scnRNA (Malone et al., 2005). 
These DCL1/scnRNA will bind with argonaute/PIWI homologues termed Twi1p (visualized first in cytoplasm). These complexes (DCL1/scnRNA/Twi1p) join HMT (Ezl1p) forming DCL1/scnRNA/Twilp/Ezl1p complexes. At this point DCL1 leaves the complexes. The final complexes are small-RNA-guided complexes (scnRNA/Twilp/Ezllp) (Mochizuki et al., 2002)(Y. Liu et al., 2007).

First, scnRNA/Twilp/Ezl1p complexes go to the parental MAC (old MAC) where scnRNAs homologous to parental MAC DNA sequences will be degraded (Mochizuki et al., 2002). Second, the remaining scnRNA/Twilp/Ezl1p complexes contain the scnRNAs that are homologous to MIC sequences. These complexes will go to the anlagen (developing MAC) and guide heterochromatin formation and subsequent DNA deletion (Mochizuki et al., 2002)(Y. Liu et al., 2007). After this, the chromodomain-1 of the programmed DNA deletion 1 protein (Pdd1p-CD1) recruits proteins involved in DNA elimination (Mochizuki et al., 2002)(Y. Liu et al., 2007).

The suggested scan RNA model for T.thermophila implies that scnRNA/Twi1p/Ezl1p will guide Ezl1p to tri-methylate the lysine 27 of H3 (H3K27me3) in the extremes of IES and perhaps the same complex will tri-methylate the lysine 9 (H3K9me3) of the interior sequences of IES (Y. Liu et al., 2007). After this, Pdd1p-CD1 will first recognize the H3K27Me2, then Pdd1p-CD1 and Pdd3p-CD will attach to H3K9Me3 marks (Taverna, Coyne, \& Allis, 2002). Pdd1p will assist with recruiting other proteins such as Lialp which is essential to complete the removal of IES (Rexer \& Chalker, 2007). Failure or lack of any of these proteins or events during conjugation will affect heterochromatin formation, DNA elimination and ultimately lead to inviable progeny (Taverna et al., 2002)(Y. Liu et al., 2007).

\subsection{Histones and Chromatin}

Eukaryotic cells package their genomic DNA in the cell nucleus. Within the nucleus, chromatin enables eukaryotic cells to compactly store a large amount of DNA. The basic unit of chromatin is the nucleosome, which is made of 147 base pairs of DNA tightly wrapped $~ 1.7$ times around a histone octamer. The canonical histone octamer is comprised of eight canonical 
histones; two H2A and H2B dimers, and one H3 and H4 tetramer (Luger, Mader, Richmond, Sargent, \& Richmond, 1997) (Figure 4). Each individual histone protein has a globular domain and $\mathrm{N}$ - and C- termini that are flexible and exposed from the nucleosome core (Luger et al., 1997). The canonical or replicative histones, which are only expressed during S-phase, are deposited into chromatin during DNA replication (Kamakaka \& Hiller, 2005). Despite their function in efficient packaging of DNA, cells must have access to DNA within chromatin to express genes or be replicated. In order to allow gene expression or DNA replication or DNA repair, the chromatin-protected region of DNA must be unraveled in a coordinated way and become available to transcription or replication factors (Aalfs \& Kingston, 2000). The noncanonical or replacement variants, which are expressed at any time during the cell cycle, are deposited into chromatin to alter nucleosome stability (Biterge \& Schneider, 2014).

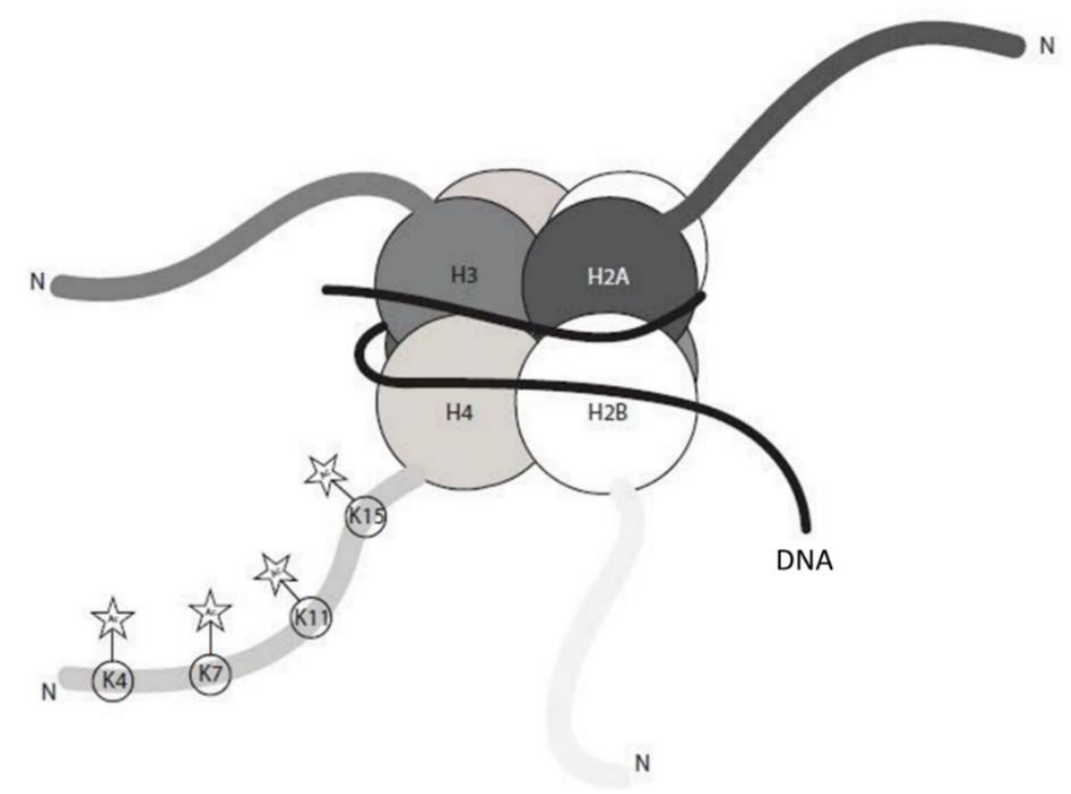

Figure 4: Nucleosome.

This representation of a nucleosome depicts the DNA wrapping the T.thermophila histone octamer. It also shows some post-translational modifications in the $\mathrm{N}$-terminal domain of Tetrahymena H4. Stars represent acetylation of lysines.

\subsection{Epigenetics}

The study of epigenetics focuses on the environmental factors that impact phenotypic trait variation. The mechanisms responsible for making these changes work by turning genes on or 
off, and by altering DNA sequences; affecting gene expression (Korochkin, 2006). Some examples of epigenetic changes are DNA methylation, as well as histone methylation, acetylation and phosphorylation, histone variant exchange, and the sliding or removing of histones within a nucleosome. Studying these epigenetic changes are important as they may be heritable, and many of these changes lead to cancer and other diseases in humans (Holliday, 2006).

\subsection{Chromatin remodeling}

Chromatin remodeling is the term used to describe the coordinated process where DNA is unwound from the histone core and exposed to transcription and replication factors (Aalfs \& Kingston, 2000). Two general types of chromatin remodeling has been described (Tang, Nogales, \& Ciferri, 2010):

\subsubsection{Post-translational modification (PTM) of histone proteins}

Post-translational modifications of histones are covalent modifications that are made by histone modifying enzymes (Berger, 2007). These post-translational modifications (PTM) are made to specific amino acid residues in a histone $\mathrm{N}$-terminal tail, globular region, or $\mathrm{C}$-terminus. Histone PTMs include 1] acetylation of lysine residues by histone acetyl transferases (HAT) such as Gcn5, and de-acetylation by histone de-acetyl transferases (HDAC). 2] Methylation of lysine or arginine residues by histone methyl transferases (HMT) such as Atxr3/Set1 and demethylation by histone de-methyl transferases (HDMT). 3] Ubiquitination of lysine by ubiquitin ligases. Ubiquitination is reversible by deubiquitylating enzymes 4] Phosphorylation and dephosphorylation of serine, threonine or tyrosine by kinases and phosphatases and 5] ribosylation of arginine by arginine adenosine-5'-diphosphoribosylation (ADP-ribosylation)(Strahl \& Allis, 2000)(Fischle, Wang, \& Allis, 2003). In some cases the PTMs can function by themselves or ciseffect. For example acetylation can negate the positive charge of the lysine side chain and result in looser attachment of the histone to negatively charged DNA. In other cases, histone modifying enzymes add PTMs to histone that are "read" by other proteins or trans-effect. For example, the human Brg1 and Saccharomyces cerevisiae (yeast) Sth1 both can recognize, or 
"read" acetylated lysines via their bromodomain (BD). Another example of readers are Drosophila HP1 or Tetrahymena Pdd1p which both read methylated lysines via their chromodomains (CD) (Strahl \& Allis, 2000)(Schwope \& Chalker, 2014).

It is important to note that histones in Tetrahymena thermophila are highly similar but not identical in their primary amino acid sequence to human histones. Based on the analysis presented in Figure 5 (EMBOSS Needle - EMBL-EBI), it can be observed that in humans the positions of some histone residues are different than the histone residues present in Tetrahymena. However, the most classic residues related to transcription in humans such as H4K5ac, H4K8ac, H4K12ac and H4K16ac (Turner, Birley, \& Lavender, 1992) are analogues to Tetrahymena H4K4ac, H4K7ac, H4K11ac and H4K15ac (See Figure 5 for pair alignments). 


\begin{tabular}{|c|c|}
\hline & Tt_H2A \\
\hline & Hs_H $2 \mathrm{~A}$ \\
\hline $\mathrm{H} 2 \mathrm{~A}$ & $\mathrm{Tt} \_\mathrm{H} 2 \mathrm{~A}$ \\
\hline & $\mathrm{HS} \_\mathrm{H} 2 \mathrm{~A}$ \\
\hline & Tt_H $2 \mathrm{~A}$ \\
\hline & $\mathrm{HS} \_\mathrm{H} 2 \mathrm{~A}$ \\
\hline & Tt_H2B \\
\hline & HS_H2B \\
\hline $\mathrm{H} 2 \mathrm{~B}$ & Tt_H2B \\
\hline & Hs_H2B \\
\hline & Tt_H2B \\
\hline & Hs_H2B \\
\hline & Tt_H3 \\
\hline & Hs_H3 \\
\hline H3 & Tt_H3 \\
\hline & Hs_H3 \\
\hline & Tt_H3 \\
\hline & Hs_H3 \\
\hline & Tt_H4 \\
\hline & Hs_H4 \\
\hline H4 & Tt_H4 \\
\hline & Hs_H4 \\
\hline & Tt_H4 \\
\hline & Hs_H4 \\
\hline
\end{tabular}

$\begin{array}{llll}1 & 5 & 8 & 12\end{array}$

MSTTGK-GGKAKGKTASSKQVSRSARAGLQFPVGRISRFLKNGRYSERIG

50 TGAPVYLAAVLEYLAAEVLELAGNAAKDNKKTRIVPRHILLAIRNDEELN 99 .|||||||||||||.||:||||||||:||||||| :|||: .||||||||||

46 AGAPVYLAAVLEYLTAEILELAGNAARDNKKTRI I PRHLQLAIRNDEELN

100 KLMANTTIADGGVLPNINPMLLPSKTKKSTEPEH----- 133

||$: \ldots||||||||||. \ldots:|||\quad| .|| \ldots \mid$

96 KLLGKVTIAQGGVLPNIQAVLLP----KKTESHHKAKGK 130

1 MAPKKAPAAAAEKKVKKAPTTE------KKNKKKRSETFAIYIFKVLKQV

$.|:.| .:|.| .|| \ldots|\ldots|: \quad|| .|:| ..|::::|::||||||$

1 -MPEPAKSAPAPKKGSKKAVTKAQKKDSKKRKRSRKESYSVYVYKVLKQV

45 HPDVGISKKAMNIMNSFINDSFERIALESSKLVRFNKRRTLSSREVQTAV 94

||$||||. .||||||||:.|| .||||||:.|:| \ldots:||||:.:|||:||| \mid$

50 HPDTGISSKAMGIMNSFVNDI FERIAGEASRLAHYNKRSTITSREIQTAV

95 KLLLPGELARHAISEGTKAVTKFSSSTN 122

$:||||||||:||:|||||||||::| \mid$.

100 RLLLPGELAKHAVSEGTKAVTKYTSSK- 126

\section{$9 \quad 27 \quad 36$}

1 MARTKQTARKSTGAKAPRKQLASKAARKSAPATGGIKKPHRFRPGTVALR 50

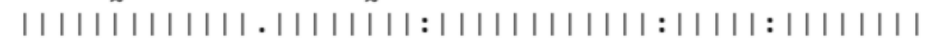

1 MARTKQTARKSTGGKAPRKQLATKAARKSAPATGGVKKPHRYRPGTVALR 50

51 EIRKYQKSTDLLIRKLPFQRLVRDIAHEFKAELRFQSSAVLALQEAAEAY 100

| | | : | | | | | : | | | | | | | | | | | | | : | | . : | । . : | | | | | | | | : | | | | | . | | .
EIRRYQKSTELLIRKLPFQRLVRE IAQDFKTDLRFQSSAVMALQEACEAT 100

51 EIRRYQKSTELLIRKLPFQRLVRE IAQDFKTDLRFQSSAVMALQEACEAT 100

101 LVGLFEDTNLCAIHARRVTIMTKDMQLARRIRGERF 136

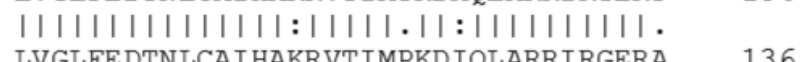

\section{Figure 5: Human and Tetrahymena histone alignments}

Tt: Tetrahymena thermophila. Hs: Human. The numbers represent the position the protein residues. The markup line between residues represent that the residues pair. Note than: in Hs_H2A lysines 9 and 13 are in position 8 and 12 respectively in Tt_H2A. Human H4 has lysines 5, 8, 12 and 16 whereas Tetrahymena has the analogues lysines 4, 7, 11 and 15. (Data retrieved from ciliate.org, NCBI and analyzed with EMBOSS Needle - EMBL-EBI) 


\subsubsection{SAGA complex}

A complex that plays a role in transcription is the HAT/transcriptional co-activator complex Spt-Ada-Gcn5-Acetyl-transferase (SAGA). This complex is primarily involved in transcription related processes, such as regulating its activation, elongation, and mRNA export (Baker \& Grant, 2007). Additionally, SAGA acetylates numerous lysine residues on the Nterminal tails of H2B and $\mathrm{H} 3$ histones. It has been proposed that Gcn5, Ada2 and Ada3 compose the catalytic core of SAGA which allows for the acetylation of histones. Defects in SAGA subunits compromise its ability to acetylate histones and regulate the activation of transcription. Misregulation of this complex in humans may lead to the development of diseases (Baker \& Grant, 2007). Abnormal functioning of the SAGA complex in humans could largely affect gene expression and development (Baker \& Grant, 2007).

\subsubsection{ATP dependent chromatin remodeling complexes}

There are two kinds of ATP dependent chromatin remodeling complexes. The first class of ATP dependent chromatin remodeling complexes recognize histones, often due to a specific pattern of histone PTM, and through ATP hydrolysis these complexes will eject or slide histones modulating DNA access (Owen-hughes, 2003)(Vignali, Hassan, Neely, \& Workman, 2000). Examples of this class are SWI/SNF and ISWI complexes. The second type of ATP dependent chromatin remodeling complexes, such as SWR, are involved in the exchange of core histones for histone variants within the nucleosome (Krogan et al., 2003). These two examples of chromatin remodeling are performed by a variety of large multi-subunit protein complexes who share the feature of having a central ATPase subunit belonging to the SNF2 superfamily of helicase-like (named after the first member of the class, yeast Swi2/Snf2 gene) (Eisen, Sweder, \& Hanawalt, 1995) (Figure 6). 
1. Post-translational modifications

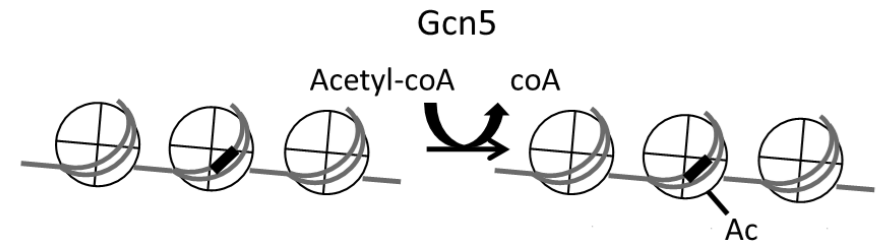

2. ATP dependent chromatin remodeling complexes

a. Histone ejectors or sliders

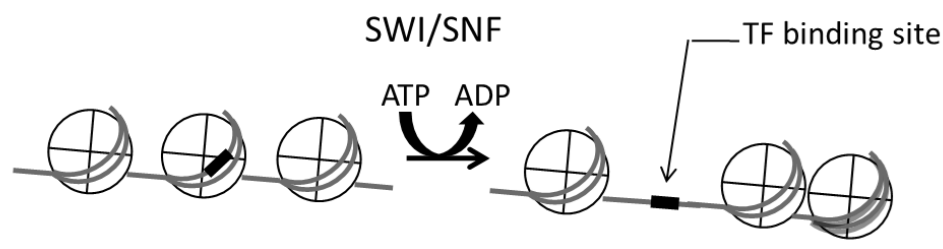

b. Histone variant exchanger

SWR-C

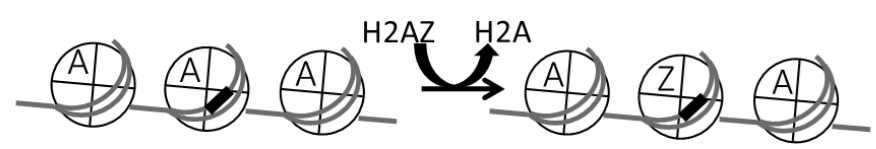

Figure 6: Mechanisms of chromatin remodeling

1; shows the histone acetyltransferase (HAT) Gen5. 2a; shows the ATP dependent chromatin remodeling complex SWI/SNF sliding a histone and exposing a transcription factor (TF) binding sites. $2 \mathrm{~b}$; shows the SWR complex replacing a H2A histones for its variant H2AZ to rapidly expose TF binding sites.

\subsubsection{SWR complex}

A known chromatin remodeling complex in eukaryotes such as humans and yeast is the SWR complex (SWR-C) (Figure 6). This complex is an ATP-dependent complex and works by exchanging variant histone dimers of $\mathrm{H} 2 \mathrm{~A} . \mathrm{Z} / \mathrm{H} 2 \mathrm{~B}$ and $\mathrm{H} 2 \mathrm{~A} / \mathrm{H} 2 \mathrm{~B}$ dimers within nucleosomes (Nguyen et al., 2013). The histone variant H2A.Z has a role in transcription, assists Exo1 nuclease throughout DNA mismatch repair, and enhances double strand break repair in DNA (Van, Williams, Kunkel, \& Peterson, 2015). The loss of this histone variant increases the prevalence of mutations in lagging strand synthesis. In Tetrahymena H2A.Z is called Hv1. Hv1 localizes to the MAC throughout T.thermophila life cycle and to the MIC only during early stages of conjugation when MIC is transcriptionally active. Hv1 is correlated with transcription (Allis et al., 1986)(Stargell et al., 1993) and it was found that loss of Hv1 is lethal (X. Liu, Li, \& GorovskyMA, 1996). Inactivation of this complex increases the number of spontaneous 
mutations in yeast and a defect in chromatin remodeling performed by the SWR-C reduces overall genome stability. The catalytic subunit of SWR-C is Swr1 (Van et al., 2015).

\subsubsection{SWI/SNF and ISWI complexes}

The SWI/SNF complex was first discovered in yeast in two separate genetic screens. The first was aimed at identifying mutants defective in yeast mating type switching (SWI) (Breeden \& Nasmyth, 1987) and the second study aimed to find mutants defective in fermentation of sucrose (SNF) (Neigeborn \& Carlson, 1984). The SWI/SNF protein complex is an ATP dependant chromatin remodeling complex which slides and ejects nucleosomes (Figure 6) (Tang et al., 2010). The main purpose of this remodeling is to unravel DNA from histones and expose the DNA to replication or transcription factors (Tang et al., 2010).

In yeast there are two different SWI/SNF-like complexes. The first is SWI/SNF and its catalytic subunit is Swi2/Snf2. This complex has a global role in transcription (Winston \& Carlson, 1992). The second yeast SWI/SNF complex is named RSC (Remodels Structure of Chromatin) and its catalytic subunit is Sth1. Swi2/Snf2 and Sth1 are highly homologous with a basic local alignment search technique for protein sequences (BLASTP) E-value of 0. The closer the E-value is to zero means that the homology across the entire length of the protein is more significant. RSC is essential for mitotic growth and it has the capacity of disturbing nucleosome structure to expose genes to transcription factors. (Cairns et al., 1996). In addition, yeast has two ISWI complexes. Each of these complexes has its own ATP-ase subunit, Iswi1 and Iswi2 respectively, which work independently and cooperatively regulating developmental responses to starvation. Isw2 in association with DNA-binding proteins controls meiosis (Kent, Karabetsou, Politis, \& Mellor, 2001).

In humans cells there exist two analogous SWI/SNF complexes, one containing Brg1 (Tang et al., 2010) and the other Brahma (Muchardt, Reyes, Bourachot, Leguoy, \& Yaniv, 1996) as catalytic subunits. Brg1 and Brahma are highly homologous (but not identical) with a BLASTP E-value of 0 . In addition, there are two ISWI complexes that have as their catalytic subunits two ATPase subunits, Snf2H and Snf2L. The ISWI complex functions are related to 
chromatin assembly, nucleosome spacing, replication, and transcriptional repression and activation (Erdel, Schubert, Marth, Längst, \& Rippe, 2010).

Tetrahymena thermophila on the other hand encodes a single Brg1/Snf2 yeast homologue. This protein may interact with other proteins that have homology to know members of the SWI/SNF complex in yeast suggesting the presence on a putative SWI/SNF in Tetrahymena. The mentioned proteins are describe in Table 1, adapted from Fillingham et al. (2006). This protein was cloned and given the name Brg1 (TTHERM_01245640) by Fillingham et al. (2006). In this paper it was also found that Brg1 localizes to MAC therefore correlates with transcription and expression of Brg1 is essential for growth. TTHERM_followed by a number represents the gene model identifier code for T.thermophila MAC genes. My searches of the sequenced MAC genome for additional Brg1-related genes were unsuccessful suggesting that a single Snf2 related protein exists in Tetrahymena. In addition, my searching of the T.thermophila sequenced MAC genome suggests it encodes two ISWI related proteins, Iswi1 (TTHERM_00388250) and Iswi2 (TTHERM_00137610). This suggests that the protist contains a single SWI/SNF with Brg1 as the catalytic subunit and two ISWI complexes with catalytic subunits Isw1 and Isw2.

The primary structure of each of catalytic subunits (Swi2/Snf2, Sth1, Brg1 and Brahma) of the SWI/SNF complex in yeast and humans contains both an ATPase domain and a bromodomain (Tang et al., 2010). The bromodomain physically interacts with acetylated histones (Reisman et al., 2009) (Figure 7). Interestingly, despite high similarity to Brg1 through most of its sequence including the ATPase domain, T.thermophila Brg1 appears to have lost its bromodomain (Fillingham et al., 2006). 


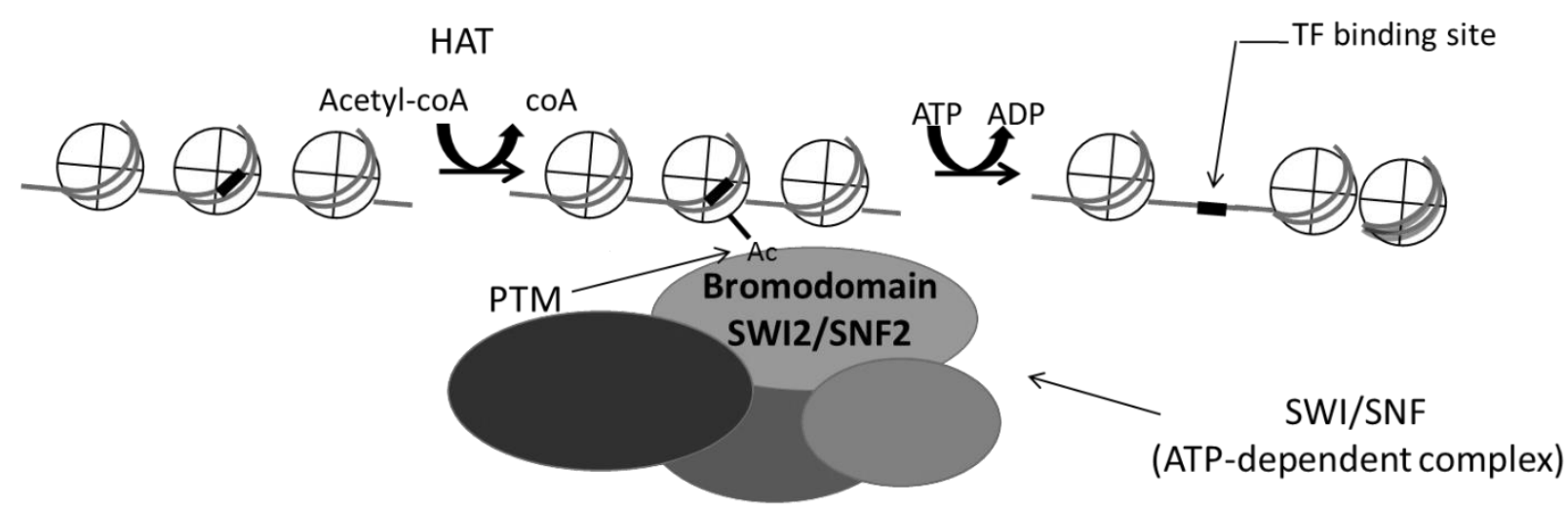

Figure 7: Bromodomains physically interacts with acetylated histones.

This figure describes that the mechanisms of chromatin remodeling are sometimes inter-related. A HAT covalently attached an acetyl group to a lysine located in the N-terminal domain of a histone. This PTM is recognized by a reader, the bromodomain of SWI2/SNF2. SWI2/SNF2 is the catalytic subunit of the SWI/SNF complex in yeast. Through ATP hydrolysis mediated by SWI2/SNF2 the SWI/SNF complex slides the histone octamer exposing the transcription factor (TF) binding site.

\subsection{SWI/SNF complex in human health}

In humans, mutations of genes encoding several members of the SWI/SNF complex (hSNF5 and BRG1) have been associated with several kinds of cancer (Roberts \& Orkin, 2004). In particular, truncating mutations of hSNF5/INI1 is related to extremely aggressive malignant rhabdoid tumors in early childhood since data suggests that hSNF5/INI1 works as a tumor suppressor. (Versteege et al., 1998). The tumor suppressor BRCA1 protein and BRG1 co-purify in the same complex of proteins and a dominat-negative mutation in $h B R G 1$ will initiate the development of cancer (Bochar et al., 2000). In addition, in multiple tumor cell lines, BRG1 is lost (Reisman et al., 2009), (Wong et al., 2000).

\subsection{Starting point to identification of SWI/SNF in Tetrahymena}

In 2006, molecular genetic analysis of Terahymena's Brgl (TtBrg1) suggest that it is a yeast $\mathrm{Brg} 1 / \mathrm{Snf} 2$ homologue; however, differently to the yeast Snf2, TtBrg1 does not have a bromodomain. In this work an antibody against Brg1/Snf2 was generated. A comparative genomic analysis of the recently sequenced Tetrahymena MAC genome predicted the existence 
of four additional members all of which are part of SWI/SNF in yeast and humans (Table 1, adapted from (Fillingham et al., 2006)). In this paper it was also found that Brg1 localizes to MAC therefore correlates with transcription and expression of Brg1 is essential for growth. The Tetrahymena Snf5 (TtSnf5) was chosen for epitope tagging since it is a well-known and conserved protein of the SWI/SNF complex in yeast and humans. Between TtSnf5 and ySnf5 there is a low BLASTP E-value of $2 \mathrm{E}^{-14}$ and with respect to hSnf5 the value is $4 \mathrm{E}^{-26}$. This suggests that between TtSnf5 and ySnf5, and TtSnf5 and hSnf5 there is a section that is highly homologous.

Table 1: Predicted subunit of the SWI/SNF complex in Tetrahymena

\begin{tabular}{|l|l|}
\hline \multicolumn{1}{|c|}{ Yeast Protein } & \multicolumn{1}{c|}{ Tetrahymena potential homologue } \\
\hline Brg1 & TTHERM_01245640 (without bromodomain) \\
\hline Swi1 & TTHERM_00243900 \\
\hline Swi3 & TTHERM_00584840 \\
\hline Snf5 & TTHERM_00304150 \\
\hline Snf12 & TTHERM_00925560 \\
\hline
\end{tabular}

\subsection{Summary}

To identify the members of the Tetrahymena SWI/SNF complex, we engineered a Tetrahymena strain which expresses epitope tagged (FZZ) Snf5 (ciliate.org TTHERM_00304150). My four independent Snf5-FZZ affinity purification experiments followed by mass spectrometry have identified the members of the first putative protist SWI/SNF complex. One protein that co-purified with Snf5 is a small bromodomain containing protein (ciliate.org TTHERM_00729230). A bromodomain is a protein domain that has affinity for acetylated lysine residues (Tamkun et al., 1992)(Cairns et al., 1996). I tagged the bromodomain containing protein (BD-FZZ) and through its affinity purification (three replicates) followed by mass spectrometry (MS) confirmed a reciprocal interaction with Snf5 as well as of most of Snf5-interacting proteins, confirming my identification of Tetrahymena SWI/SNF complex. Additionally, I found that the bromodomain containing protein is versatile in that it interacts with a set of proteins that likely originate from several additional chromatin remodeling 
complexes, specifically Tetrahymena SAGA, SWR and a HMT. I have named this Snf5copurifying bromodomain-containing protein Interactive ㅁromodomain protein1 (Ibd1). In order to determine whether the bromodomain of Ibd1 is functional we expressed Ibd1 as a recombinant protein and found it specifically interacted with acetyl-lysine containing histone peptides. Interestingly, our data suggest that Ibd1 may recognize two or more PTM at one time rather than individual PTM. Finally, my indirect immunofluorescence (IF) demonstrates that Ibd1 localizes to the MAC during growth and sexual development consistent with a role in transcription.

The initial rationale for functional analysis of Iswi1 (TTHERM_00388250) was based on its publically available gene expression profile that suggested a role in DNA rearrangements of Tetrahymena. In order to begin to get an idea of whether this was the case, I therefore engineered Tetrahymena to express epitope tagged Iswi1 (Iswi1-FZZ). My Western blot analysis shows that as expected Iswi1 is expressed when DNA rearrangements occur, but also during vegetative growth and starvation. My indirect IF data shows that Iswil localizes to the transcriptionally silent MIC in growth and sexual development consistent with a role in mitosis/meiosis or maintenance of silent chromatin. MS data from two AP experiments of Iswi1-FZZ shows that it interacts with several proteins the significance of which at this point is unknown. 


\section{MATERIALS AND METHODS}

\subsection{Strain generation - Construction of gene specific 3xFLAG-TEV-ZZ (FZZ) targeting vectors and transgenic Tetrahymena}

The predicted coding region of genes encoding proteins epitope tagged in this study were assessed by BLASTP to ensure clear, conserved SWI/SNF and ISWI orthologues with homology across the length of the protein particularly the C-terminus. Any BLASTP E-value that was lower than $1 \mathrm{E}^{-3}$ was accepted as sufficient molecular identity to proceed with tagging. It is important to know that in T.thermophila, chromosomal integration of transgenes predominantly occurs by homologous recombination (Cassidy-Hanley et al., 1997). The approach used in this study was to introduce a DNA sequence encoding the epitope tag itself as well as 3`sequences to ensure proper transcription of the tagged gene linked to a selectable marker.

The vector pBKS-FZZ (Figure 8) was used to engineer the tagging cassette as it possesses DNA sequence encoding the FZZ epitope tag. This vector contains an ampicillin-resistance cassette (for initial selection and subsequent propagation in bacteria), two unique restriction enzyme recognition polylinker sites (to sub-clone in the desired upstream and downstream homologous sequences to guide proper integration of the epitope tag). The tagging cassette contains:

1. DNA sequence encoding an epitope tag FZZ. The epitope tag FZZ contains: A] a three times repeated FLAG sequence (3xFLAG). FLAG is a polypeptide with the following sequence DYKDDDDK. (Knappik \& Pluckthun, 1994). The 3xFLAG is recognized by the monoclonal and commercially available M2 antibody and the anti-FLAG M2 affinity gel agarose beads. B] TEV (Tobacco Etch Virus protease cleavage site) sequence. $\mathrm{C}$ ] ZZ sequence (domain of protein $\mathrm{A}$, recognized by $\mathrm{IgG}$ sepharose beads and some secondary IgG antibodies).

2. At the 3' end of the epitope FZZ there is a RPL29 3'untranslated region (RPL29 3' UTR) which contains the in frame TGA stop codon and a transcriptional terminator signal (polyA signal). This RPL29 3' UTR is not technically part of the tag but is 
required for the proper transcription of the tagged gene since when the cassette is integrated, the normal 3'UTR of the tagged gene is essentially unlinked from the gene so this replaces it (Kataoka, Schoeberl, \& Mochizuki, 2010).

3. A neo2 chimeric gene that when expressed in Tetrahymena confers resistant to paromomycin to successful T.thermophila transformants. The neo2 chimeric gene includes the neomycin resistance gene as the open reading frame (ORF). The ORF is expressed under the control of the Tetrahymena H41 promoter and includes a TGA stop codon followed by a transcriptional terminator signal (BTU1 3' UTR) (K. Collins, 2012). H41 is an active histone promoter which directs a high level of the neomycin gene transcription ensuring resistance to paromomycin (K. Collins, 2012).

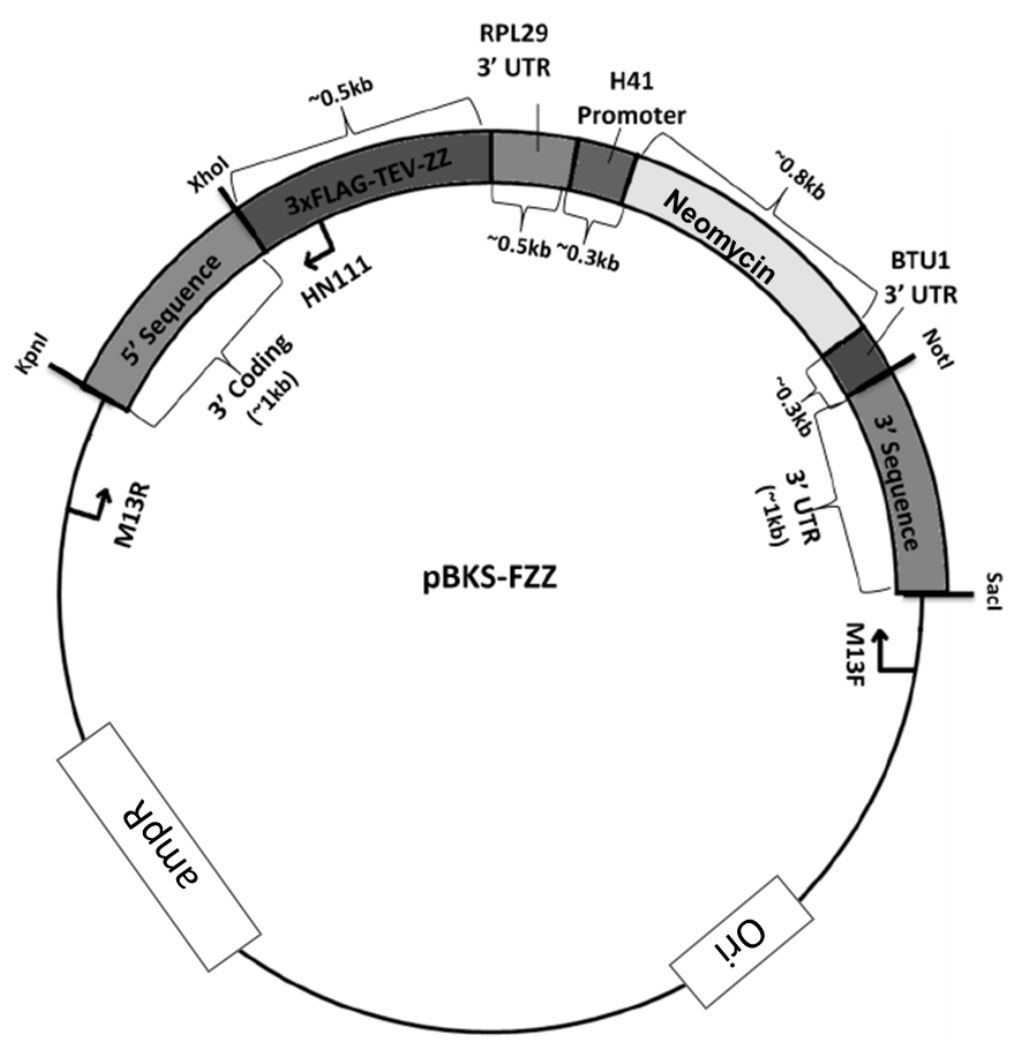

Figure 8: pBKS-FZZ plasmid MAP. (Garg et al., 2013a)

Since the T.thermophila MAC can be genetically manipulated and it is possible to fuse the tagging construct to the DNA sequence encoding the C-terminus of the protein of interest using the homologous recombination machinery of the cell. Tagging constructs were introduced into 
the Tetrahymena MAC using particle bombardment. Particle bombardment is a technique used to transfer genetic material into organisms by shooting coated metal particles at the target cells. This is done using a biolistic device or a gene gun. Ultimately after selection and phenotypic assortment a strain which can express from its own promoter the desired protein fused in-frame to the epitope FZZ is generated. Note the strain can still express the tagged protein before phenotypic assortment which is used to generate a homozygous MAC (Cassidy-Hanley et al., 1997). To accomplish my research goals I used a previously generated SNF5-FZZ strain and I engineered "tagging constructs" and transgenic strains for genes coding for Ibd1 (former BD protein) and Iswi1.

\subsubsection{Tetrahymena thermophila cell strains, culture, cells collection, starvation and mating}

The T.thermophila cell strains used for transformation and as wild type controls were B2086 [Mpr+/Mpr+ (mp-s, mt II)] and CU428 [Mpr/Mpr (mp-s, mt VII)]. The CU428 strain is homozygous for alleles that confer resistance to 6-methylpurine $(6 \mathrm{mp})$ in the diploid MIC; however, in the MAC it carries only the allele that is sensitive to this drug. Thus $6 \mathrm{mp}$ selection can be used to select for exconjugants of a mating involving CU428. Both strains have the rDNA gene that is sensitive to paromomycin. (Gaertig, Gu, Hai, \& Gorovsky, 1994) and therefore can be transformed with neo 2 based plasmids used in this study. These strains were obtained from the Tetrahymena stock center of Cornell University, Ithaca NY.

\subsubsection{Bench culture}

These strains were stored in our lab at room temperature as bench cultures, in axenic and sterile conditions, in reusable $25 \times 150 \mathrm{~mm}$ rimless, glass test tubes. Each tube contain $10 \mathrm{~mL}$ of sterile 1xSPP (sequestrine protease peptone) medium (see Appendix 1) and was covered with reusable $25 \mathrm{~mm}$ two way plastic test tube cap to allow gas exchange. I will refer to the mentioned system as test tube cultures. The maintenance of bench cultures was performed every month and consisted in transferring $1 \mathrm{~mL}$ of the previous culture into a new tube containing $9 \mathrm{~mL}$ of sterile $1 x$ SPP. 


\subsubsection{Tetrahymena thermophila vegetative growth (Overnight culture)}

For small (below 10mL) and starter cultures, Tetrahymena thermophila cells were grown to $\log$ phase $\left(2 \times 10^{5}-3 \times 10^{6}\right.$ cells $\left./ \mathrm{mL}\right)$ in test tube cultures in an incubating shaker (MaxQ 4450 Benchtop, Thermo Scientific) at $30^{\circ} \mathrm{C}$ at $100 \mathrm{rpm}$ for 15 hours. For large cultures, I added the starter culture in sterile flasks containing the required volume of 1xSPP media. The cells were grown to log phase using the previous described conditions in a larger incubator with shaker (MaxQ 5000 Incubated/Refrigerated Floor Shaker - Analog, Thermo Scientific). In order to allow efficient aeration, the media volume to flask size ratio was 1:10.

\subsubsection{Cell collection and washing}

For small cultures, $1 \mathrm{~mL}$ or $10 \mathrm{~mL}$ of overnight culture were transferred into a microcentrifuge tube $(1.5 \mathrm{~mL}$ Boilproof Microtube, Frogga Bio) or a conical tube $(15 \mathrm{~mL}$ conical tubes, PP, Frogga Bio) and to keep the cells alive I slowly spun the micro centrifuge tubes (5424 Eppendorf) or the conical tubes (Allegra X-15R, Beckman Coulter) at room temperature, at 3000 rpm for 4 minutes. To wash the cells, the media was quickly removed by air vacuum aspiration, before cells start swimming, trying not to disturb the cell pellet. The cells were re-suspended and washed with $1 \mathrm{~mL}$ or $5 \mathrm{~mL}$ of $10 \mathrm{mM}$ Tris- $\mathrm{HCl} \mathrm{pH} 7.4$ (starvation buffer) (see Appendix 1) accordingly and the washing and spinning process repeated followed by air vacuum aspiration. This pellet can be stored at $-80^{\circ} \mathrm{C}$ for later use or re-suspended in starvation buffer for immediate use. For large cultures cells of $250 \mathrm{~mL}$, I transferred $250 \mathrm{~mL}$ of overnight cultures into centrifuge bottles (250mL Centrifuge Bottle PPCO, Fisher Scientific) and spun the bottles (RC5C Plus

Centrifuge, Sorvall) at room temperature for 4 minutes at 3000rpm. The media was quickly removed by inversion, before cells start swimming, trying not to disturb the cell pellet. The cells were re-suspended and washed with $100 \mathrm{~mL}$ of $10 \mathrm{mM}$ Tris- $\mathrm{HCl} \mathrm{pH} 7.4$ (see Appendix 1). The washing and spinning process was repeated followed by removal of the buffer by inversion. This pellet can be stored at $-80^{\circ} \mathrm{C}$ for later use or re-suspended in starvation buffer for immediate use. 


\subsubsection{Cells starvation}

The collected and washed cells can be starved in the same amount of $10 \mathrm{mM}$ Tris- $\mathrm{HCl} \mathrm{pH}$ 7.4 (starvation buffer) used for growth. The cells must be starved for 24 hours at $30^{\circ} \mathrm{C}$ without shaking. Cells can be collected at this stage as described above for further analysis, wash is not required since they are in starvation buffer, or they can be used for mating. This pellet can be stored at $-80^{\circ} \mathrm{C}$ for later use or re-suspended in starvation buffer for immediate use.

\subsubsection{Cells mating}

The same amount of starved cells (for 24 hours) from different mating types, for example B2086 and CU428, were mixed and incubated at $30^{\circ} \mathrm{C}$ without shaking. Cells can be collected at this stage as described above for further analysis, wash is not required since they are in starvation buffer. This pellet can be stored at $-80^{\circ} \mathrm{C}$ for later use or re-suspended in starvation buffer for immediate use.

\subsubsection{Genomic DNA isolation}

The used method for T.themophila genomic DNA extraction is described by Gaertig et al., 1994. A volume of $10 \mathrm{~mL}$ of overnight culture of the B2086 strain were grown and collected as described above.

The cell pellet was re-suspended in $500 \mu \mathrm{L}$ of Tetrahymena lysis solution (see Appendix 1) using a micro-pipette and this solution was transferred to a $1.5 \mathrm{~mL}$ micro-centrifuge tube. To remove the proteins and lipids, $600 \mu \mathrm{L}$ of phenol:chloroform $(1: 1)$ was added and rotated for one minute at room temperature (Rotorack 343, Fisher Scientific) until a homogeneous opaque colour was reached. The micro-centrifuge tube was spun at room temperature for 1 minute at 13000rpm (5424 - Eppendorf). The top layer was transferred to a new micro-centrifuge tube

making sure that only the top layer was transferred and the middle layer was not disturbed. This step was repeated one more time. To the new tube containing the top layer from the previous step, the same amount of chloroform was added, it should be approximately $600 \mu \mathrm{L}$, and rotated 
for one minute at room temperature until a homogeneous opaque colour was reached. The micro-centrifuge tube was spun at room temperature for 1 minute at $13000 \mathrm{rpm}$. The top layer was transferred to a new micro-centrifuge tube. This step was repeated one more time. To the new micro-centrifuge tube with approximately $600 \mu \mathrm{L}$ of the sample, $200 \mu \mathrm{L}$ of $5 \mathrm{M} \mathrm{NaCl}$ (see Appendix 1) was added. This has a sample and $5 \mathrm{M} \mathrm{NaCl}$ ratio of 3:1. This solution was mixed by inversion 10 times. To precipitate the DNA, $800 \mu \mathrm{L}$ of isopropanol, corresponding to the same volume as the previous total volume, was added. The sample was rotated for one minute at room temperature. The micro-centrifuge tube was spun at room temperature for 2 minutes at 13000rpm. The supernatant was discarded by inversion. To the same tube and to remove the isopropanol, $200 \mu \mathrm{l}$ of $70 \%$ ethanol was added. This solution was mixed by inversion 10 times followed by spinning at room temperature for 2 minutes at 13000rpm. The supernatant was discarded by inversion. This step was repeated one more time. The sample was dried using a Speed Vac System (Speed Vac System, Labconco) at room temperature for 30 minutes to remove ethanol residues. To re-suspend the DNA pellet, $100 \mu \mathrm{L}$ of sterile $\mathrm{ddH}_{2} \mathrm{O}$ was added and incubated at $37^{\circ} \mathrm{C}$ for 1 hour.

\subsubsection{Sequence data retrieval and primers design}

In order to design the primers, the gene sequences which code for the Snf5-interacting bromodomain-containing protein (TTHERM_00729230) and Iswi1 (TTHERM_00388250) were obtained from the Tetrahymena Genome Database Wiki (TGD Wiki) was used (ciliate.org). The Institute for Genomic Research (TIGR) of Bradly University, Peoria IL, sequenced the Tetrahymena thermophila genome and uploaded it onto a database called TGD Wiki. This database can be updated by each user and contains information on Tetrahymena genes and proteins from sources such as scientific literature and the research community.

To engineer FZZ-tagged strains, primers were designed to amplify two separate sequences for each desired gene of T.thermophila genomic MAC DNA. The first sequence was a $1 \mathrm{~kb}$ genomic MAC region (1kbUP) located directly upstream and in-frame with, but not including, the wild type stop codon. The second sequence was an additional $1 \mathrm{~kb}$ genomic MAC region $(1 \mathrm{kbDOWN})$ located immediately downstream of the wild type stop codon. The function 
of these two sequences is to guide the "tagging construct" to the two complementary MAC gene sequences of interest in order to replace the WT stop codon for the FZZ and the neo2 chimeric gene by same gene replacement. . The first pair of primers, for the $1 \mathrm{kbUP}$ (Appendix 3), have at their 5 end restriction enzyme recognition sites for $K p n I$ and XhoI. The second pair of primers (Appendix 3), for the $1 \mathrm{kbDOWN}$, have at their 5 ends restriction enzyme recognition sites for NotI and SacI. These two PCR generated sequences were inserted after appropriate restriction enzyme digestion into the vector (Figure 8) one at the time (tandem) using molecular cloning methods and transformation into Escherichia coli DH5- $\alpha$.

\subsubsection{PCR on genomic Tetrahymena DNA}

The final volume used for PCR reactions was $50 \mu \mathrm{L} ; \mathrm{H}_{2} \mathrm{O}$ added to complete $50 \mu \mathrm{L}, 1 \mathrm{U}$ of DNA polymerase and its buffer (Phusion High-Fidelity, Thermo Scientific), 0.2mM of dNTPs, $0.5 \mu \mathrm{M}$ of each primer (Appendix 3) and 1pg-10ng of DNA template. The PCR reaction was prepared in a PCR tube. (0.2mL thin-wall flat cap PCR tubes, MaxyClear Axygen). The PCR reactions were performed in a thermocycler (Perkin-Elmer Geneamp 9600) using the program illustrated in Table 2.

Table 2: PCR program to amplify the gene targets.

\begin{tabular}{|c|l|c|c|}
\hline Cycles & \multicolumn{1}{|c|}{ Program } & Temperature $\left({ }^{\mathbf{0}} \mathbf{C}\right)$ & Time (seconds) \\
\hline 1 & Initial denaturation & 95 & 300 \\
\hline 35 & Denaturation & 95 & 10 \\
\cline { 2 - 4 } & Annealing & 50 & 15 \\
\cline { 2 - 4 } & Elongation & 72 & 60 \\
\hline 1 & Final elongation & 72 & 600 \\
\hline
\end{tabular}

To assess if the PCR was successful, electrophoresis was employed. The PCR products were run in a mini 1xTBE $0.8 \%$ agarose gel (see Appendix 1) at $80 \mathrm{~V}$ for 30 minutes (PowerPac Universal, BioRad). The gel was visualized by an imaging system (Gel Doc XR System, BioRad). 


\subsubsection{Escherichia coli growth}

From our long term storage, cells are in glycerol stocks kept at $-80^{\circ} \mathrm{C}$, E.coli cells containing the plasmid pBKS-FZZ with the cloned in $~ 800 \mathrm{bp}$ UP and $\sim 800 \mathrm{bp}$ DOWN sequences for the Asf1 gene were streaked into LB + ampicillin $(100 \mu \mathrm{g} / \mathrm{mL})$ plates and incubated overnight at $37^{\circ} \mathrm{C}$. A single colony was inoculated in a sterile culture tube $(14 \mathrm{~mL}$ round bottom, polypropylene tube, dual-position snap cap, Falcon) containing $3 \mathrm{~mL}$ of $\mathrm{LB}+$ ampicillin and shaken overnight at $225 \mathrm{rpm}$ at $37^{\circ} \mathrm{C}$ (MaxQ 4450 Benchtop, Thermo Scientific). The $3 \mathrm{~mL}$ of culture was used for plasmid isolation.

\subsubsection{Plasmid isolation, pBKS-FZZ plasmid and PCR products restriction digestion, ligation, transformation and plasmid sequencing}

The two PCR generated sequences, corresponding to the $1 \mathrm{kbUP}$ and $1 \mathrm{kbDOWN}$ stream of the stop codon from BD (TTHERM_00729230) and ISWI1 (TTHERM_00388250) genes, were inserted after appropriate restriction enzyme digestion into the vector one at the time using molecular cloning methods and transformation into E.coli (DH5- $\alpha$ ).

\subsubsection{Plasmid isolation}

A $3 \mathrm{~mL}$ culture of E.coli, containing the plasmid pBKS-FZZ, grown overnight was used for plasmid isolation with a miniprep kit (High-Speed Plasmid Mini Kit, Geneaid). To assess if the isolation was successful, the kit eluates were run in a mini 1xTBE $0.8 \%$ agarose gel (see Appendix 1) at $80 \mathrm{~V}$ for 30 minutes (PowerPac Universal, BioRad). The gel was visualized by an imaging system (Gel Doc XR System, BioRad). 
2.1.6.2 pBKS-FZZ plasmids and 1kbUP PCR products restriction digestion followed by fragment recovery

The 1kbUP BD (TTHERM_00729230), 1kbUP ISWI1 (TTHERM_00388250) PCR products and the pBKS-FZZ plasmids were digested with KpnI and XhoI (Life Technologies) following the manufacturer specifications.

The digested PCR products were cleaned (Gel/PCR DNA Fragments Extraction Kit, Geneaid) and the digested plasmid were run in a mini 1xTAE 0.8\% agarose gel (see Appendix 1) at $40 \mathrm{~V}$ for 80 minutes (PowerPac Universal, BioRad). The gel was visualized and cut with a new razor under UV light (Short/long-wave UV lamp, Cole-Parmer). The digested plasmid in the cut gel was extracted (Gel/PCR DNA Fragments Extraction Kit, Geneaid).

\subsubsection{DNA ligation and transformation}

The digested and cleaned 1kbUP BD (TTHERM_00729230) and 1kbUP Iswi1 (TTHERM_00388250) PCR products were separately ligated to the digested and cleaned pBKSFZZ plasmids following the ligase (T4 DNA Ligase, Life Technologies) manufacturer recommendations. Both ligated products were transformed into competent E.coli DH5- $\alpha$ (Subcloning Efficency DH5- $\alpha$ Competent cells, Life Technologies) following the manufacturer instructions and usage of transformation controls for each experiment. After transformation, the E.coli cells were spread into LB + ampicillin $(100 \mu \mathrm{g} / \mathrm{mL})$ plates and incubated overnight at $37^{\circ} \mathrm{C}$. Six single colonies from each experiment were inoculated in six sterile culture tubes ( $14 \mathrm{~mL}$ round bottom, polypropylene tube, dual-position snap cap, Falcon) containing $3.5 \mathrm{~mL}$ of $\mathrm{LB}+$ ampicillin and shaken overnight at $225 \mathrm{rpm}$ at $37^{\circ} \mathrm{C}$ (MaxQ 4450 Benchtop, Thermo Scientific). The $3 \mathrm{~mL}$ of culture was used for plasmid isolation (High-Speed Plasmid Mini Kit, Geneaid) to verify if the colonies contained a plasmid, the rest was stored at $4^{0} \mathrm{C}$ for further inoculation.

To verify if these plasmids were the pBKS-FZZ plasmids and contained the corresponding $1 \mathrm{kbUP}$ PCR product, $2 \mu \mathrm{L}$ of plasmids from each experiment were double digested 
with KpnI and XhoI (Life Technologies) following the manufacturer specifications. The rest was stored at $-20^{\circ} \mathrm{C}$ for sequencing.

To verify if the released product was $100 \%$ identical to the required sequence, the isolated plasmids (undigested), corresponding to the digested plasmid, were sequenced by an external provider (ACGT Corp.), by Sanger using the generic primer HN111 (See Appendix 3). The obtained sequence was aligned (Nucleotide EMBOSS Needle, EMBL-EBI) to the gene sequence obtained from ciliate.org. The previously stored cells at $4^{0} \mathrm{C}$ corresponding to this experiment were growth for plasmid isolation for the subsequent addition of the $1 \mathrm{kbDOWN}$ stream of the stop codon sequence.

In order to insert the 1kbDOWN BD (TTHERM_00729230), 1kbDOWN Iswi1 (TTHERM_00388250), the same steps described above were followed. The restriction enzymes used were NotI and SacI (Life Technologies) and the generic primer used for Sanger sequencing was M13F (See Appendix 3). The final arrangement of sequence on the "tagging construct" within the vector was be KpnI-1kbUP-FZZ-RPL29 3'UTR-NEO2-1kbDOWN-SacI. The cells containing the pBKS-FZZ plasmid with the required tagging construct were grown overnight. A volume of $800 \mu \mathrm{L}$ of the overnight culture was mixed with $800 \mu \mathrm{L}$ of $50 \%$ glycerol (sterile) and stored at $-80^{\circ} \mathrm{C}$.

\subsubsection{Tagging construct recovery}

A volume of $30 \mathrm{~mL}$ of E.coli cells containing the plasmids with the "tagging constructs" for Iswi1 (TTHERM_00388250) and BD (TTHERM_00729230) were grown and the plasmids were isolated using 10 miniprep per gene. The cell input per miniprep was $3 \mathrm{~mL}$. The obtained plasmid were combined obtaining $500 \mu \mathrm{L}$ of miniprep DNA [50 $\mathrm{gg}$ ]. The miniprep DNA was digested as follows; in a $1.5 \mathrm{~mL}$ micro-centrifuge tube $100 \mu \mathrm{L}$ miniprep DNA [10 $\mu \mathrm{g}], 40 \mu \mathrm{L}$ of buffer, $8 \mu \mathrm{L}$ of $\mathrm{KpnI}$ and $8 \mu \mathrm{L}$ of $\mathrm{SacI} \mathrm{H}_{2} \mathrm{O}$ to complete $400 \mu \mathrm{L}$ were added and incubated at $37^{\circ} \mathrm{C}$ for 16 hours. To assess if the double digestion was successful, $2 \mu \mathrm{L}$ of each digested product was run in a mini 1xTBE $0.8 \%$ agarose gel (see Appendix 1) at $80 \mathrm{~V}$ for 50 minutes (PowerPac Universal, BioRad). The gel was visualized by an imaging system (Gel Doc XR System, 
BioRad). The final arrangement of sequence on the "tagging cassette" within the vector were KpnI-1kbUP-FZZ-RPL29 3'UTR-NEO2-1kbDOWN-SacI. Prior to T.thermophila transformation, the plasmid in E.coli with the construct needed to be digested with restriction enzymes (KpnI and SacI) to free the homologous ends. For transformation into T.thermophila, 4$5 \mu \mathrm{g}$ of digested and purified DNA was needed.

The digested genes were cleaned using phenol-chloroform extraction. The digestion $(400 \mu \mathrm{L})$ reaction was re-suspended with the same amount of phenol:chloroform (1:1) and rotated for one minute at room temperature (Rotorack 343, Fisher Scientific) until a homogeneous opaque colour was reached. The micro-centrifuge tube was spun at room temperature for 1 minute at $13000 \mathrm{rpm}$ (5424 - Eppendorf). The top layer was transferred to a new micro-centrifuge tube. To the new tube containing the top layer from the previous step, the same amount of chloroform was added, it should be approximately $400 \mu \mathrm{L}$, and rotated for one minute at room temperature until a homogeneous opaque colour was reached. The microcentrifuge tube was spun at room temperature for 1 minute at $13000 \mathrm{rpm}$. The top layer was transferred to a new micro-centrifuge tube. This step was repeated one more time. To the new micro-centrifuge tube with approximately $400 \mu \mathrm{L}$ of the sample, $40 \mu \mathrm{L}(1: 10)$ of commercially available $3 \mathrm{M}$ NaOAc was added. This solution was mixed by inversion 10 times. To precipitate the DNA, $1 \mathrm{~mL}$ of $100 \%$ cold ethanol, corresponding to 2.5 times the volume than the previous $400 \mu \mathrm{L}$ volume, was added. The sample was inverted 10 times and stored at $-20^{\circ} \mathrm{C}$ overnight. The micro-centrifuge tube was spun at $4^{\circ} \mathrm{C}$ for 15 minutes at $13000 \mathrm{rpm}$ (22R Refrigerated Microcentrifuge, Beckman Coulter). The supernatant was discarded by inversion. To the same tube and to remove the $100 \%$ ethanol, $1.4 \mathrm{~mL}$ of $70 \%$ cold ethanol was added. This solution was mixed by inversion 10 times followed by spinning at $4^{0} \mathrm{C}$ for 15 minutes at $13000 \mathrm{rpm}$ (22R Refrigerated Microcentrifuge, Beckman Coulter). The supernatant was discarded by inversion. The sample was dried using a Speed Vac System (Speed Vac System, Labconco) at room temperature for 30 minutes to remove ethanol residues. To re-suspend the DNA pellet, $12 \mu \mathrm{L}$ of sterile ddH2O was added and incubated at $37^{\circ} \mathrm{C}$ for 1 hour. A volume of $1 \mu \mathrm{L}$ was run in an agarose gel to assess if the extraction was successful. The remaining sample was stored at $-20^{\circ} \mathrm{C}$ to use in T.thermophila transformation. 


\subsection{Transformation of T.thermophila}

Biolistics (Bruns \& Cassidy-Hanley, 1999) was used to transform T.thermophila MAC by gene replacement. A total volume of 50mL of B2086 and CU428 cells (different mating types, for mating experiments) were grown to log phase and starved as described above.

\subsubsection{Biolistics}

The gold particles $(1 \mu \mathrm{m}$ Gold Microcarriers, BioRad) were coated with $5 \mu \mathrm{g}$ of the linearized DNA (digested plasmid, $1 \mu \mathrm{g} / \mu \mathrm{L}$ ). To attach the DNA onto the gold particles, $1 \mathrm{M}$ of calcium chloride and $10 \mathrm{mM}$ of spermidine (Sigma) were pre-chilled and added to $1 \mu \mathrm{m}$ gold particles and DNA in a microcentrifuge tube. This solution was mixed using a vortex for 15 minutes at $4^{0} \mathrm{C}$. The solution was spun for 10 seconds at maximum speed and the supernatant was removed. A volume of $200 \mu \mathrm{L}$ of $70 \%$ ice cold ethanol was added and span for 10 seconds at maximum speed, the supernatant was removed. A volume of $200 \mu \mathrm{L}$ of $100 \%$ ice cold ethanol was added and span for 10 seconds at maximum speed, the supernatant was removed. The DNA coated gold particles were re-suspended with $20 \mu \mathrm{L}$ of $70 \%$ ice cold ethanol. The gene gun (PDS1000/He Biolistic particle delivery system, BioRad) apparatus and parts were washed with $\mathrm{ddH}_{2} \mathrm{O}$ and $70 \%$ ethanol and air dried. The $50 \mathrm{~mL}$ of starved B2086 and CU428 cells were spun at room temperature for 4 minutes at 3000rpm. The starvation buffer was aspirated and resuspended in $1 \mathrm{~mL}$ of fresh starvation buffer. The gene gun was assembled and the cells located into the gene gun as described (Bruns \& Cassidy-Hanley, 1999). The DNA coated gold particles were shot into the cells. The shot cells were transferred to $50 \mathrm{~mL}$ of pre-warmed to $30^{\circ} \mathrm{C}$ SPP media and incubated at $30^{\circ} \mathrm{C}$ at $100 \mathrm{rpm}$ for 3 hours for cell recovery.

\subsubsection{Cell passages and phenotypic assortment}

To the $50 \mathrm{~mL}$ of recovered cells, paromomycin (Paromycin Sulfate, Calbiochem) to a final concentration of $100 \mu \mathrm{g} / \mathrm{mL}$ was added. The $50 \mathrm{~mL}$ of cells containing paromomycin was transferred to 96 well plates (Tissue culture plate 96-well flat bottom suspension cells, Sarstedt), $200 \mu \mathrm{L}$ per well, and incubated at $30^{\circ} \mathrm{C}$ for 3 days. After this period, the cells were observed 
using a microscope to locate growing cells. The growing cells are cells where identical gene replacement by homologous recombination occurred. After this initial selection, paromomycin concentration was gradually increased, to accelerate phenotypic assortment, up to $1 \mathrm{mg} / \mathrm{mL}$. This process is to replace all endogenous copies of the targeted gene with the FZZ-tagged versions. These cells were stored in bench cultures.

\subsubsection{Trichloroacetic acid precipitation and Western blotting (TCA-WB)}

Trichloroacetic acid (TCA) precipitation followed by Western blotting analysis was used to assess if paromomycin resistant strains were expressing the tagged protein.

\subsubsection{TCA precipitation}

The cell extracts were prepared using 10\% TCA precipitation (Bright, Kambesis, Nelson, Jeong, \& Turkewitz, 2010). In a microcentrifuge tube $1 \mathrm{~mL}$ of $\log$ phase, starved or mating cells were collected, as described in cells collection, re-suspended in $90 \mu \mathrm{L}$ of starvation buffer. A volume of $10 \mu \mathrm{L}$ of $100 \%$ TCA was added to obtain a final concentration of $10 \%$ TCA. The lysisprecipitation solution remained in ice for 20 minutes. The micro-centrifuge tube was spun at room temperature for 2 minutes at 13000rpm (5424 - Eppendorf) and the supernatant was discarded. The pellet containing soluble and insoluble proteins was re-suspended in $90 \mu \mathrm{L}$ of SDS loading dye (see Appendix 1). To neutralize the solution $10 \mu \mathrm{L}$ of $1 \mathrm{M}$ Tris (see Appendix 1) was added. The samples were incubated in boiling water for 5 minutes. A volume of $10 \mu \mathrm{L}$ of each sample was loaded into sodium dodecyl sulfate-polyacrylamide gels (SDS gels) and the rest stored at $-20^{\circ} \mathrm{C}$ for further analysis.

\subsubsection{Western blotting}

Western blot is a simple and efficient procedure based on the electrophoretic transfer of proteins from SDS gels to unmodified nitrocellulose and detection by enhanced chemiluminescence (ECL) of specific antibodies (Burnette, 1981). The SDS gels for SDS-PAGE were prepared as follows; the stacking part of the gel was 4\% (see Appendix 1) and the running 
portion was either $10 \%$ or $15 \%$. A volume of $10 \mu \mathrm{L}$ of samples was loaded $\left(2 \times 10^{5}-3 \times 10^{6}\right.$ cells/mL) to be resolved either with PiNK Plus Prestained Protein Ladder (FroggaBio) or BLUeye Prestained Protein Ladder (FroggaBio) (see Appendix 4). The gels were run (MiniPROTEAN Tetra Handcast Systems, BioRad) in 1xSDS-running buffer (see Appendix 1) at a constant voltage of $100 \mathrm{~V}$ for 2.5 hours (PowerPac Universal, BioRad). The separated proteins were transferred (Trans-Blot Cell, BioRad) to a $0.45 \mu \mathrm{m}$ nitrocellulose membrane (BioRad) using transfer buffer (see Appendix 1) at a constant voltage of 80V for 2.5 hours (PowerPac Universal, BioRad). The nitrocellulose membrane was blocked in 3\% skim milk (BioShop) (see Appendix 1) shaking (The belly dancer, Stovall) for one hour at room temperature or overnight at $4^{0} \mathrm{C}$. After blocking, the nitrocellulose membrane was washed 3 times for 5 minutes each wash in 1xTBS (see Appendix 1) shaking at room temperature (The belly dancer, Stovall). The nitrocellulose membrane was incubated with different antibodies as indicated in Table 3 . Following manufacturer specifications, ECL detection (WestarNova 2011, Cyanagen) and visualization were performed (Gel Doc XR System, BioRad).

Table 3: Conditions for incubation of Antibodies

\begin{tabular}{|c|c|c|c|}
\hline Primary antibody & Washes & Secondary antibody & Washes \\
\hline $\begin{array}{l}\text { 1:4000; mouse monoclonal to } \\
\text { FLAG (Sigma-Aldrich): } \\
\text { Primary antibody buffer (see } \\
\text { Appendix 1). } 1 \text { hour incubation } \\
\text { with shaking }\end{array}$ & $\begin{array}{l}3 \text { times for } 5 \\
\text { minutes each } \\
\text { wash in } \\
\text { 1xTBS with } \\
\text { shaking }\end{array}$ & $\begin{array}{l}\text { 1:5000; HRP-conjugated goat } \\
\text { polyclonal to mouse } \\
\text { (Cedarlane):3\% skim milk (see } \\
\text { Appendix 1). } 45 \text { minutes } \\
\text { incubation with shaking }\end{array}$ & $\begin{array}{l}3 \text { times for } 15 \\
\text { minutes each } \\
\text { wash in } \\
\text { 1xTBS with } \\
\text { shaking }\end{array}$ \\
\hline $\begin{array}{l}\text { 1:1000; rabbit polyclonal to } \\
\text { Brg1 (In house): Primary } \\
\text { antibody buffer (see Appendix } \\
\text { 1). } 1 \text { hour incubation with } \\
\text { shaking }\end{array}$ & $\begin{array}{l}3 \text { times for } 5 \\
\text { minutes each } \\
\text { wash in } \\
\text { 1xTBS with } \\
\text { shaking }\end{array}$ & $\begin{array}{l}\text { 1:5000; HRP-conjugated goat } \\
\text { polyclonal to rabbit } \\
\text { (Cedarlane):3\% skim milk (see } \\
\text { Appendix 1). } 45 \text { minutes } \\
\text { incubation with shaking }\end{array}$ & $\begin{array}{l}3 \text { times for } 15 \\
\text { minutes each } \\
\text { wash in } \\
\text { 1xTBS with } \\
\text { shaking }\end{array}$ \\
\hline $\begin{array}{l}\text { 1:2500; rabbit polyclonal to } \\
\text { Pdd1 (abcam): Primary } \\
\text { antibody buffer (see Appendix } \\
\text { 1). } 1 \text { hour incubation with } \\
\text { shaking }\end{array}$ & $\begin{array}{l}3 \text { times for } 5 \\
\text { minutes each } \\
\text { wash in } \\
\text { 1xTBS with } \\
\text { shaking }\end{array}$ & $\begin{array}{l}\text { 1:5000; HRP-conjugated goat } \\
\text { polyclonal to rabbit } \\
\text { (Cedarlane):3\% skim milk (see } \\
\text { Appendix 1). } 45 \text { minutes } \\
\text { incubation with shaking }\end{array}$ & $\begin{array}{l}3 \text { times for } 15 \\
\text { minutes each } \\
\text { wash in } \\
\text { 1xTBS with } \\
\text { shaking }\end{array}$ \\
\hline
\end{tabular}




\subsection{Affinity purification - Western blotting (AP-WB)}

The 3xFLAG sequence is recognized by the monoclonal and commercially available M2 antibody and the anti-FLAG M2 affinity gel agarose beads. The epitope FZZ is normally used for tandem affinity protein purification which involves two sequential steps of affinity purification. First, affinity purification with IgG sepharose beads followed by TVE protease and a second purification using anti-FLAG M2 affinity gel agarose beads. In this research, only one step of affinity purification using ANTI-FLAG M2 affinity gel agarose beads was used.

\subsubsection{Whole cell extraction}

Nuclear proteins isolation requires relatively high salt concentrations. The concentration of $\mathrm{NaCl}$ used was $300 \mathrm{mM}$. This is relatively high, high enough to lyse the nuclei and free genomic DNA which will make the lysed cells extremely viscous. A cell pellet of less than $3 \mathrm{~mL}$ in a $50 \mathrm{~mL}$ centrifuge tube from $500 \mathrm{~mL}(2 \times 250 \mathrm{~mL})$ of overnight culture or mating cells was used as starting material. Frozen pellets were kept cold during the thawing procedure. Cells pellets thaw relatively slowly. The procedure required room temperature water in a big beaker. The tubes containing the samples were swirled for 30 seconds and then placed on ice, this step was repeated until the cells pellet were totally thawed. On ice, an equal volume to the cells pellet of ice cooled 2x lysis buffer (see Appendix 1) containing protease inhibitors for yeast (SigmaAldrich) and $200 \mu 1$ of $100 \mathrm{mM}$ PMSF were added and mixed by gently pipetting up and down. At this point the solution became viscous due to the DNA. The solution was adjusted to a total volume of $15 \mathrm{~mL}$ using $1 \times$ lysis buffer (see Appendix 1) containing protease inhibitors for yeast (Sigma-Aldrich) and $200 \mu \mathrm{l}$ of $100 \mathrm{mM}$ PMSF and mixed by gently pipetting up and down. A volume of $300 \mu \mathrm{l}$ of $10 \% \mathrm{NP} 40$ to $0.2 \%$ final concentration were added. A volume of $5 \mu 1$ of Benzonase (Sigma-Aldrich) to digest all the released genomic DNA was added. Viscosity of the whole cell extraction (WCE) was tested using a cut blue tip and a P1000. There was viscosity present at this point. The tube was gently inverted 10 times and left to rotate (Rotorack 343, Fisher Scientific) at $4^{\circ} \mathrm{C}$ for 1 hour to allow benzonase to digest all the released genomic DNA. Viscosity of the WCE was tested as mentioned above. Viscosity disappeared at this point. To clarify, this step gets rid of insoluble proteins, the samples by centrifugation of the $15 \mathrm{~mL}$ of 
WCE were divided into $101.5 \mathrm{~mL}$ pre-chilled micro centrifuge tubes and spun (22R Refrigerated Microcentrifuge, Beckman Coulter) at $4^{\circ} \mathrm{C}$ at maximum speed for 30 minutes. This step was repeated one more time by transferring supernatant by inversion to fresh pre-chilled micro centrifuge tubes. The clarified WCE (supernatants) were pooled from all tubes into a single $50 \mathrm{~mL}$ pre-chilled centrifuge tube on ice and spun (Allegra X-15R, Beckman Coulter) at $4^{\circ} \mathrm{C}$ at 4750rpm for $6 \mathrm{~min}$ to separate remaining insoluble proteins, carbohydrates and lipids. The supernatant was transferred to a new pre-chilled $50 \mathrm{~mL}$ centrifuge tube using a $25 \mathrm{~mL}$ pipette, on ice, and the pellet was discarded. A volume of 50 $\mu$ l of WCE were saved in a separate pre-chilled microcentrifuge tube at $-80^{\circ} \mathrm{C}$ to be used as input material for Western blotting.

\subsubsection{Immunoprecipitation}

Affinity purification (AP), affinity chromatography or immunoprecipitation (IP) permits the preparation of adsorbents (agarose beads) containing ligands (FLAG antibody) attached by bonds to the tagged proteins and this tagged protein is attached to its complex. The FLAG antibodies bonds are susceptible to specific chemical breakage using ammonium hydroxide $\left(\mathrm{NH}_{4} \mathrm{OH}\right)$, thus providing means of removing the intact protein-ligand complex from the affinity adsorbent (Cuatrecasas, 1970). In other words, affinity chromatography using agarose beads with FLAG antibodies is a method which facilitates the separation of FLAG tagged proteins from whole cell extracts (WCE) and because native condition are used during this separation, other proteins that bind the tagged protein will co-purify as well. It is based on the highly specific interaction between the 3x-FLAG antigen, attached to the protein of interest, and the FLAG antibody, linked to the agarose beads plus native conditions during the proteins extraction. The immunoprecipitation (IP) protocol followed was designed specifically for T.thermophila which works accordingly (Garg et al., 2013b). In addition, from these samples a small part were used for western blotting to A] confirm and assess quality of AP by amount of bait recovered and B] to determine of TtBrg1 co-purifies as was done for Snf5-FZZ (Figure 1). Affinity chromatography was done at different physiological stages such as vegetative growth and conjugation. The rationale for this is that during vegetative growth (MAC is active) the T.thermophila SWI/SNF complex is expected to be interacting with a certain set of proteins. Fillingham et al. (2006) previously showed that TtBrg1 was required for progression through 
conjugation. Therefore, I am interested to determine if the Tetrahymena SWI/SNF complex interacts with additional or different proteins than yeast/humans during growth and conjugation. Futher analysis of any specific newly identified protein(s) will predict functions.

A volume of $100 \mu 1$ of M2 agarose slurry (ANTI-FLAG M2 Affinity Gel, Sigma Aldrich) was used for each sample. This $100 \mu 1$ of M2 agarose slurry corresponds to $50 \mu 1$ of M2 agarose beads. Using a cut tip, the required slurry containing the beads was added to a pre-chilled $15 \mathrm{~mL}$ centrifuge tube and washed with $5 \mathrm{~mL}$ of cold 1 xlysis buffer and slowly inverted 5 times to resuspend beads. The $15 \mathrm{~mL}$ centrifuge tube containing the slurry and cold 1xlysis buffer was spun at $4^{\circ} \mathrm{C}$ at $3000 \mathrm{rpm}$ for 2 minutes and the supernatant was aspirated. The washing of the beads was repeated two more times. After the last aspiration the beads were equilibrated with an equal amount of 1xlysis buffer to the beads alone. Using a cut tip, $100 \mu 1$ per sample of the new slurry containing the equilibrated beads was transferred to pre-chilled $15 \mathrm{~mL}$ centrifuge tubes, one per sample. The pooled supernatants were added to the respective $15 \mathrm{~mL}$ falcon tubes containing the $\mathrm{M} 2$ beads and rotated at $4^{\circ} \mathrm{C}$ for 3 hours. The tubes were spun at $4^{\circ} \mathrm{C}$ at $3000 \mathrm{rpm}$ for 2 minutes. The supernatant was aspirated and discarded. The beads containing the tagged proteins and interacting proteins were washed 1 time with 10mL of IPP300 (see Appendix 1), 2 times with $5 \mathrm{~mL}$ of IPP100 (see Appendix 1) and 2 times with 5mL of IPP100 without detergent (see Appendix 1). Between every wash the samples were rotated at $4^{\circ} \mathrm{C}$ for 5 minutes, spun at $4^{\circ} \mathrm{C}$ at 3000rpm for 2 minutes and the supernatant was aspirated and discarded. For the final wash, $750 \mu$ l of cold $\mathrm{CaCl}_{2}$ /Tris solution (see Appendix 1) was added. Using a cut tip the slurries were transferred to microcentrifuge tubes and mixed by inversion 5 times. The microcentrifuge tubes were spun at room temperature at 5000rpm for $1 \mathrm{~min}$ and the supernatant carefully removed using a micropipette. To separate the beads from the tagged proteins and interacting proteins, $500 \mu 1$ of freshly made $\mathrm{NH}_{4} \mathrm{OH}$ (see Appendix 1) were added, this is a denaturing condition. The microcentrifuge tubes were rotated at room temperature for $20 \mathrm{~min}$ and spun at room temperature at 5000rpm for $2 \mathrm{~min}$. The supernatant contains the proteins were transferred to new pre-chilled microcentrifuge tubes and $50 \mu \mathrm{l}$ (IP) from this tube were transferred to separate pre-chilled microcentrifuge tubes for Western blotting. The remaining $450 \mu \mathrm{l}$ were used for mass spectrometry (MS). Both samples were stored at $-80^{\circ} \mathrm{C}$ until use. 


\subsubsection{Western blotting}

The purpose of this step is to use Western blot analysis to confirm that affinity purification was successful. The used samples are the input from WCE and IP from immunoprecipation. The samples that showed good recovery of bait were used for MS. The employed method and antibodies are described above.

\subsection{Mass spectrometry and SAINTexpress analysis}

Mass spectrometry was used to identify the bait protein and any co-purifying proteins in our AP experiments. The approach for this step is to use the eluates from the affinity purification step and analyze these samples using mass spectrometry (AP-MS) to identify sets of co-purifying proteins for each bait protein. Mass spectrometry is an analytical method that is used to measure and determine the presence and abundance of specific compounds by separation through mass/charge ratio. For example, based on the unique spectra of molecules we can determine compounds such as peptides. Each spectra is based on the individual and specific molecule's mass present within a sample (Fenn et al., 2013). In other words, mass spectrometry will facilitate the determination of the bait-pray interactions or the presence of any polypeptides that are co-purifying with FZZ-tagged proteins (bait).

FZZ-tagged proteins (bait) and any co-purifying proteins (prey) were digested with trypsin and were loaded to a C18 column to be separated by reversed-phase high-pressure liquid chromatography (RP-HPLC). The RP-HPLC column had C18 (hydrophobic) as static phase and $2 \%$ acetonitrile and $0.1 \%$ formic acid as mobile phase. This column was attached to the LTQ Ion Trap Mass Spectrometer to create a continuous system. All these mentioned steps were performed by the Gingras laboratory. The Mass Spectrometer outputs are spectra. The obtained spectra was identified and further filtered by a MASCOT (search engine) score to obtain spectral counts. Spectral count sums (SpecSum) represent the number of proteins in a complex mixture by providing the total number of mass spectra that match peptides to a particular protein. 
The SAINTexpress (Significance Analysis of INTeractome express) algorithm, developed by the Choi, Nesvizhskii and Gingras laboratories, was used to transform the MASCOT SpecSum to SAINTexpress SpecSum. The obtained SAINTexpress SpecSum represents a given bait-prey interaction into the probability the physical interaction is significant (Skarra et al., 2011). SAINTexpress eliminates background data to obtain a reproducible set of proteins that copurify with a given bait. Therefore SAINTexpress filters out false positive from non-specific binding proteins since the algorithm incorporates all available purification data from other tagged proteins as well as more than 30 untagged control purifications or mock IP. In other words, mock IP is important for SAINTexpress because it shows that co-purification of the protein is a direct consequence of the tagged protein and not unspecific binding to the chromatography resin. In conclusion, SAINTexpress converts spectral counts for a specific bait-prey interaction into the probability that it represents a true interaction (100\%) which must pass a set high cut-off value to be included in a SAINTexpress curated data table. After SAINTexpress, the set of proteins that co-purify with the given bait are expected. Meaning, the proteins found are interacting with the FZZ-tagged (bait) proteins. The AP-MS was repeated two or more times for each bait to have a rigorous idea of the set of co-purifying proteins.

Mass spectrometry and SAINTexpress were performed by Dr. Jean Philippe Lambert in the collaborating laboratory of Dr. Ann-Claude Gingras in the Lunenfield-Tanenbaum Research Institute in the Mount Sinai Hospital in Toronto as described in the literature (Teo et al., 2014). The eluates that have the separated FZZ proteins plus any co-purifying proteins were analyzed by mass spectrometry (Garg et al., 2013) as follows. Affinity purified proteins were eluted in $0.5 \mathrm{M} \mathrm{NH}_{4} \mathrm{OH}$ and were taken to dryness in a speed-vac without heat. The proteins were digested with trypsin (Kean, Couzens, \& Gingras, 2012). The resulting peptides were manually bomb loaded onto a capillary column and placed in the LTQ (Linear ion-Trap Quadrupole) mass spectrometer equipped with an HPLC (High Performance Liquid Chromatography) Agilent 1100 capillary (Garg et al., 2013) followed by MASCOT and SAINTexpress as described in the literature (Teo et al., 2014). 


\subsection{Indirect immunofluorescence}

Indirect immunofluorescence (IF) was used to identify where in the cell these FZZ tagged proteins (bait) are localized. I harvested and fixed the cells for IF using the method of Wenkert and Allis (Wenkert \& Allis, 1984). The nuclear counterstaining was with 4,6-diamidino-2phenylindole dihydrochloride (DAPI) for fluorescence microscopy (Garg et al., 2013b).

In a microcentrifuge tube $1 \mathrm{~mL}$ of washed cells in different physiological stages were spun at room temperature at $12000 \mathrm{rpm}$ for 2 minutes. The starvation buffer was removed by aspiration to leave $500 \mu \mathrm{L}$ of it. A volume of $1.7 \mu \mathrm{L}$ of Shandin (see Appendix 1) was added to the samples to fix the cells. The sample was spun at room temperature at 10000rpm for 1 minute and the supernatant was discarded. A volume of $100 \mu \mathrm{L}$ of cold acetone was added, inverted 10 times and placed on ice for 5 minutes. The sample was spun at room temperature at 10000rpm for 1 minute and the supernatant was discarded. A volume of $500 \mu \mathrm{L}$ of $1 \mathrm{xPBS}$ (see Appendix 1) was added, inverted 10 times and spun at room temperature at 10000rpm for 1 minutes and the supernatant was discarded. The primary antibodies were mixed on 1xPBST (see Appendix 1) in a 1:1000 ratio and $100 \mu \mathrm{L}$ was added. The samples were rotated at room temperature for 1 hour. The sample was spun at room temperature at 5000rpm for 2 minutes and the supernatant was discarded. The samples were re-suspended in $500 \mu \mathrm{L}$ of $1 \times \mathrm{xBS}$, spun at room temperature at 5000rpm for 2 minutes and the supernatant was discarded. The fluorescently conjugated

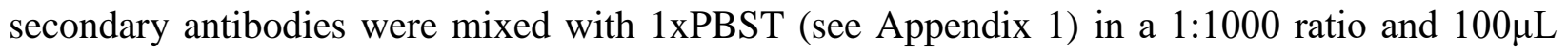
was added. The samples were rotated at room temperature for 1 hour in the dark. The sample was spun at room temperature at 5000rpm for 2 minutes and the supernatant was discarded. The samples were re-suspended in $500 \mu$ Lof $1 \times$ PBS, spun at room temperature at 5000rpm for 2 minutes and the supernatant was discarded. A volume of $100 \mu \mathrm{L}$ of DAPI (4',6-diamidino-2phenylindole) solution was added and incubated in the dark for 5 minutes. A total of $20 \mu \mathrm{L}$ of each sample was mounted on slides with coverslips and epi-fluorescence examined on a compound, upright Leica DM5000B microscope under magnification of 400x. 


\subsection{Comprehensive analysis of SWI/SNF complex}

To characterize the possible function of the SWI/SNF complex during vegetative growth and conjugation, I used the data obtained from the SAINTexpress analysis of the specific newly identified protein(s) and predicted their functions based on functions of orthologous proteins present in other organisms. These proteins were grouped in putative complexes using Venn diagrams.

\subsection{Synthetic gene - cloning and expression of Tetrahymena thermophila Interactive Bromodomain Protein 1 (Ibd1)}

In order to begin to understand the function of Ibd1, it was necessary to determine if its bromodomain recognizes a specific histone post-translational modification. In order to accomplish this, a recombinant protein was engineered.

\subsubsection{DNA optimization}

Tetrahymena uses a non-universal genetic code where UAA and UAG specify glutamine and not stop codons. Therefore in order to express a full length Ibd1 protein in E.coli, we engineered a synthetic gene to use appropriate codons for glutamic acid. In addition, we took the opportunity to optimize codon usage for expression in E.coli BL21. The engineering of the synthetic gene was performed by an external provider (BioBasics Inc.) The gene was provided in a pUC57 plasmid.

\subsubsection{Strain generation}

\subsubsection{Amplification of Synthetic Ibd1}

The Ibd1 synthetic gene was amplified using polymerase chain reaction (PCR). To amplify Ibd1, primers were designed carrying at their 5 end restriction enzyme recognition sites to facilitate its cloning into pET28a. Specifically, the forward primer contained an NdeI site, and 
the reverse primer a BamHI site (Appendix 3). The template was a 1:1000 dilution of the pUC57 plasmid mentioned above. The PCR conditions are outlined in the PCR section.

2.7.2.2 Restriction digestion, ligation into expression vector and transformation into E.coli DH5 $\alpha$

The PCR products and an expression pET28 plasmid were double digested with NdeI and BamHI (Life Technologies) following the manufacturer specifications. To assess if the digestion was successful, $2 \mu \mathrm{L}$ of the digested products were run in a mini 1 xTBE $0.8 \%$ agarose gel (see Appendix 1) at $80 \mathrm{~V}$ for 20 minutes (PowerPac Universal, BioRad). The gel was visualized by an imaging system (Gel Doc XR System, BioRad). The obtained image of the gel (Figure X) shows that the digestion was successful. The PCR products were cleaned, the plasmids gel purified, and both parts ligated as described above. The ligated DNA was transformed into E.coli DH5 $\alpha$ strains and selected for transformants using media containing the antibiotic kanamycin. The transformed cells were streaked onto a plate with media containing kanamycin, allowing for the selection of transformants with kanamycin resistance. The success of the cloning was confirmed by sequencing. Plasmid DNA was purified, analyzed using restriction enzyme digestion and agarose gel electrophoresis, and sequenced by an external provider (M13F and M13R generic primers (See Appendix 3)) to verify that no mutations were introduced by PCR and that the gene was cloned into the correct position of the pET28 vector, in-frame with DNA sequence that when translated will add a N-terminal 6x His-tag to Ibd1. The rest of the plasmids were used for transformation into E.coli BL21 (DE3).

\subsubsection{Transformation into E.coli BL21 (DE3)}

Next the sequence verified plasmid was transformed into E.coli BL21 (DE3) (New England Bio Labs) following the manufacturer specifications. Positive transformants were grown in media containing kanamycin +/- IPTG. Whole cell extracts by sonication, SDS loading dye following by incubation in boiling water were made. The samples were separated by SDSPAGE, and visualized by staining with Fast SeeBand protein staining solution in order to determine if recombinant 6xHIS-Ibd1 protein expressed at the correct size. 


\subsubsection{Inducing Expression of Ibd1 in E. coli BL21 (DE3)}

The addition of IPTG was required to induce transcription and displace the lac repressor from the operator site in the host E.coli BL21 (DE3) cells. The cells were grown overnight at $37^{\circ} \mathrm{C}$. The next day, I re-suspended them in liquid culture and incubated them until the $\mathrm{OD}_{600}$ reached approximately 0.6 . The culture was then induced with IPTG for 4 hours. I took samples at 1 hour time intervals starting from time 0 to 4 hours post induction.

\subsubsection{Purification of Expressed 6XHis-Ibd1}

Extraction of the 6XHis-Ibd1 was done using Ni-NTA Fast Start Kit made (Qiagen). The recombinant protein was purified under native conditions. First, $3 \mathrm{~mL}$ cell pellet from E.coli BL21 (DE3) culture were obtained and suspended it in native Lysis Buffer. The lysate was centrifuged to ensure cellular debris was contained in the pellet. The supernatant was retained, as this contained the soluble portion of the recombinant protein. A volume of $5 \mu \mathrm{L}$ of the supernatant was aliquoted and kept on ice. The nickel containing resin was re-suspended in a Fast Start Column by inversion. The seal was broken and allowed for the storage buffer to drain out before adding the cell lysate supernatant to the column. The flow through fraction was collected and a $5 \mu \mathrm{L}$ aliquot was obtained. The column was washed twice, each time with $4 \mathrm{~mL}$ of native wash buffer. A $5 \mu \mathrm{L}$ aliquot was taken after each wash. With the addition of two $1 \mathrm{~mL}$ aliquots of Native elution buffer, the bound 6XHis-Ibd1 was eluted from the column. The wash and elution buffers used had low concentrations of imidazole added to act as a competitive inhibitor, binding to the nickel and promoting the elution of the $6 \mathrm{XHis}-\mathrm{Ibd} 1$. I collected $5 \mu \mathrm{L}$ of each flow through, wash and elution fraction in separate tubes and mixed them with $5 \mu \mathrm{L}$ of SDS loading dye and boiled them for 5 minutes. I used SDS PAGE to verify the success of each elution. 


\subsubsection{Buffer Exchange by Size Exclusion Chromatography}

Buffer exchange is based on size exclusion chromatography (PD-10 Desalting Columns, GE Healthcare). The objective of this step is to obtain the 6XHis-Ibd1 in a buffer free from imidazole, appropriate for downstream applications. Elutions 1 and 2 from the Ni-NTA purification were passed through the column. The protein of interest, 6XHis-Ibd1, was large enough to enter the pores of the resin and was therefore eluted from the column in the new buffer. This elution was obtained in different fractions. Each of the 8 samples were run in an SDS-PAGE, followed by staining of the gel by Fast SeeBand Protein stain solution (FroggaBio).

\subsection{Histone Peptide Array}

A commercial human histone peptide array (MODified Histone Peptide Array, Active Motif Catalog number 13005) and its reagents (MODified Protein Domain Binding Kit, Active Motif Catalog number 13007) was used to screen Ibd1 for binding interactions with 384 unique posttranslational modification combinations. The array contained 384 sites, each with a different combination of PTM. The mechanism of the array was comparable to a Western blot. The array was blocked with 5\% milk for 1 hour, washed with 1xTBS and then incubated with my protein of interest, 6XHis-Ibd1, for 1 hour. The array was washed with 1xTBS and incubated first with the primary antibody; anti-6XHis, and then with the secondary antibody; anti-rabbit HRP. After washing again with $1 \mathrm{X}$ TBS, ECL was used for detection. The array was visualized by an

imaging system (Gel Doc XR System, BioRad). The obtained image was processed in the software provided with the kit (Array Analyze Software, Active Motif). The output of the software was used to analyze each of the sites with binding interactions. 


\section{CHAPTER 1 - MOLECULAR CLONING OF TAGGING CONSTRUCTS AND GENERATION OF TAGGED LINES RESULTS}

\subsection{Genomic DNA isolation}

I purified whole cell genomic DNA from WT Tetrahymena in order to use as template for PCR amplification of homologous sequence that were cloned in pBSK-FZZ. Volumes of 2, 5 and $10 \mu \mathrm{L}$ were run in an agarose gel to assess if the extraction was successful. The remaining sample was stored at $-20^{\circ} \mathrm{C}$ for use in PCR (Polymerase Chain Reaction). To assess if the extraction was successful, the DNA was run by electrophoresis. The obtained image of the gel (Figure 9) shows that both extractions were successful and contain DNA.

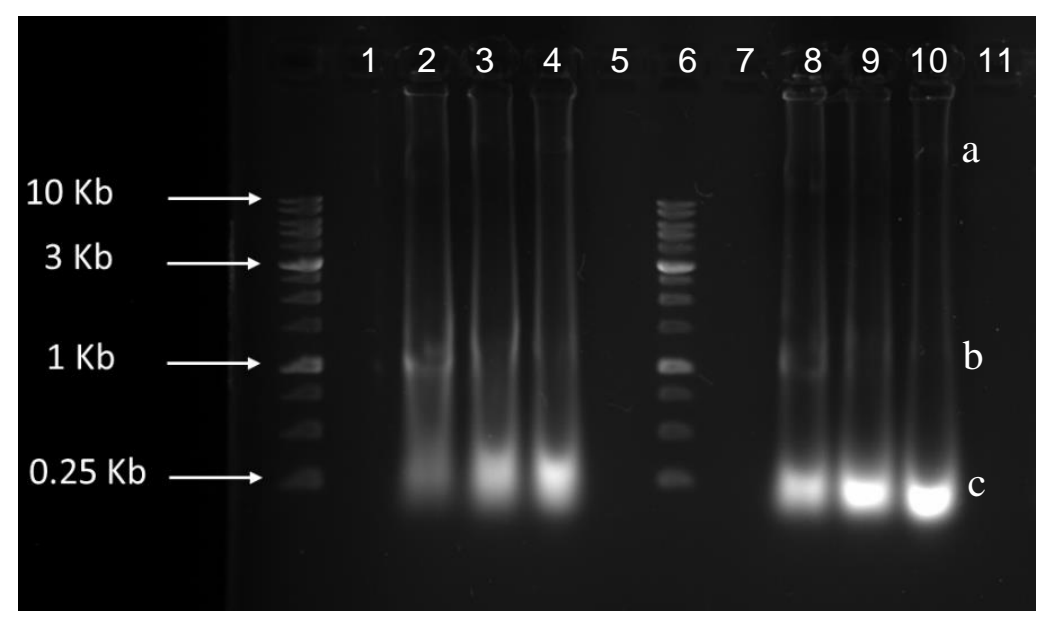

Figure 9: Two DNA isolations from whole cell genomic DNA.

First DNA Isolation: lane $1,4 \mu \mathrm{L}$ ladder; lane 2 , empty; lane $3,4 \mu \mathrm{L}$ loaded $(2 \mu \mathrm{L}$ blue loading dye $+2 \mu \mathrm{L}$ DNA); lane $4,7 \mu \mathrm{L}$ loaded $(2 \mu \mathrm{L}$ blue loading dye $+5 \mu \mathrm{L}$ DNA $)$; lane $5,7 \mu \mathrm{L}$ loaded $(2 \mu \mathrm{L}$ blue loading dye $+10 \kappa \mathrm{DNA})$; lane 6 , empty. Second DNA Isolation: lane $7,4 \mu \mathrm{L}$ ladder; lane 8 , empty; lane $9,4 \mu \mathrm{L}$ loaded $(2 \mu \mathrm{L}$ blue loading dye $+2 \mu \mathrm{L}$ DNA); lane 10, $7 \mu \mathrm{L}$ loaded $(2 \mu \mathrm{L}$ blue loading dye $+5 \mu \mathrm{L}$ DNA $)$; lane $11,7 \mu \mathrm{L}$ loaded $(2 \mu \mathrm{L}$ blue loading dye $+10 \mu \mathrm{L}$ DNA); lane 12 , empty. a. DNA; b and c. RNA. 


\subsection{PCR on genomic Tetrahymena DNA}

Next I amplified UP and DOWN sequence for Ibd1 and ISW1 genes. The obtained image of the gel (Figure 10) shows that the four reactions; 1 for UP Ibd1, 1 for DOWN Ibd1, 1 for UP ISWI1 and 1 for DOWN ISW1 were successful and contain the expected PCR product of approximately $1 \mathrm{~kb}$. The remaining sample was separated from PCR Master Mix (Gel/PCR DNA Fragments Extraction Kit, Geneaid), the PCR products recovery was assessed by electrophoresis. The cleaned and recovered products were stored at $-20^{\circ} \mathrm{C}$ to be used for enzyme digestion.

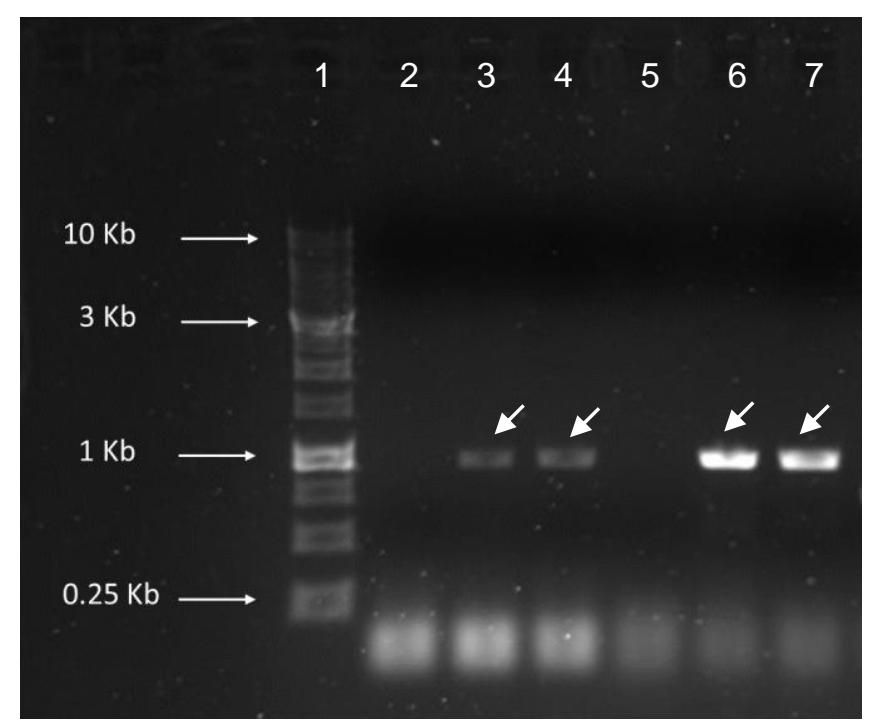

Figure 10: PCR on genomic Tetrahymena DNA for BD (TTHERM_00729230) and Iswi1 (TTHERM_00388250).

BD (TTHERM_00729230) PCR: lane 1, 4 $\mu \mathrm{L}$ ladder; lane 2, negative control (water as template); lane 3; 1kbUP; lane 4, 1kbDOWN. Iswi1 (TTHERM_00388250) PCR: lane 5, negative control (water as template); lane 6,1 kbUP; lane 7, $1 \mathrm{kbDOWN}$. $5 \mu \mathrm{L}$ of every mixed sample was loaded $(2 \mu \mathrm{L}$ blue loading dye $+5 \mu \mathrm{L}$ PCR products). The arrows show the PCR products.

\subsection{Plasmid isolation}

PCR amplified UP and DOWN sequence for Asf1 were cloned into pBSK-FZZ prior to this thesis work. The obtained image of the gel (Figure 11) shows that the 12 plasmid isolation of pBSK-FZZ were successful since the band corresponding to the plasmids are running at the expected size $\sim 9 \mathrm{~kb}$. 


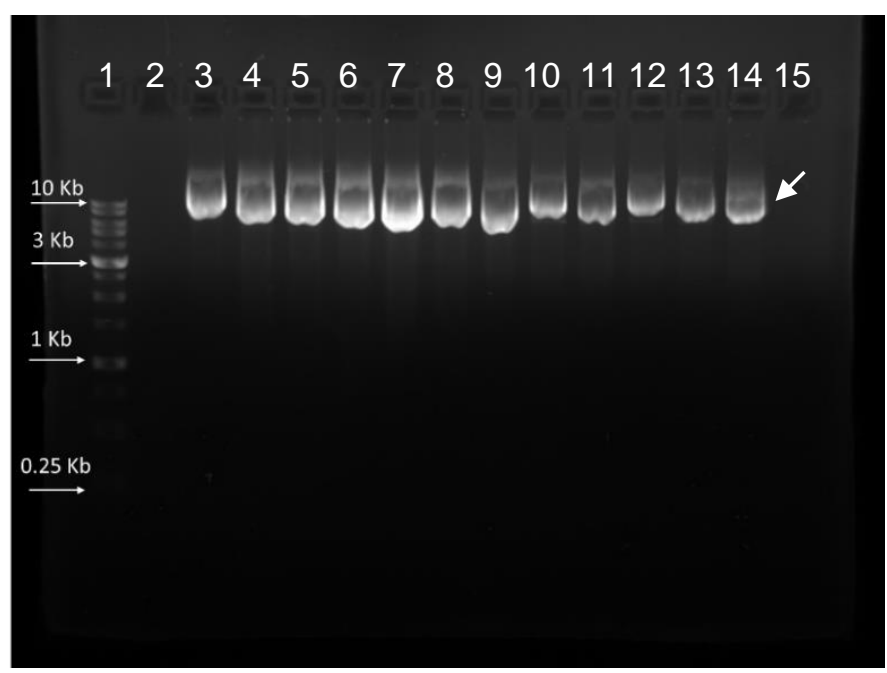

\section{Figure 11: Plasmid pBSK-FZZ isolation.}

Lane 1, $4 \mu \mathrm{L}$ ladder; lane 2, empty; lane 3 - 14, plasmids; lane 15, empty. The arrow shows the 12 plasmid isolation of pBSK-FZZ. The bands correspond to the plasmids and are running at the expected size $\sim 9 \mathrm{~kb}$.

\section{4 pBKS-FZZ plasmids and 1kbUP PCR products restriction digestion followed by fragment recovery}

Two plasmids containing the cloned in UP and DOWN ( 800bp) sequences for Asf1, UP BD PCR product and UP ISWI1 PCR product were double digested with KpnI and XhoI. To assess if the digestion was successful, $2 \mu \mathrm{L}$ of the digested products were assessed by electrophoresis The obtained image of the gel (Figure 12) shows that the digestion was successful since we were able to observe that the two plasmids containing the cloned in Asf1 gene released the $\sim 800 \mathrm{bp}$ corresponding to the UP region. 


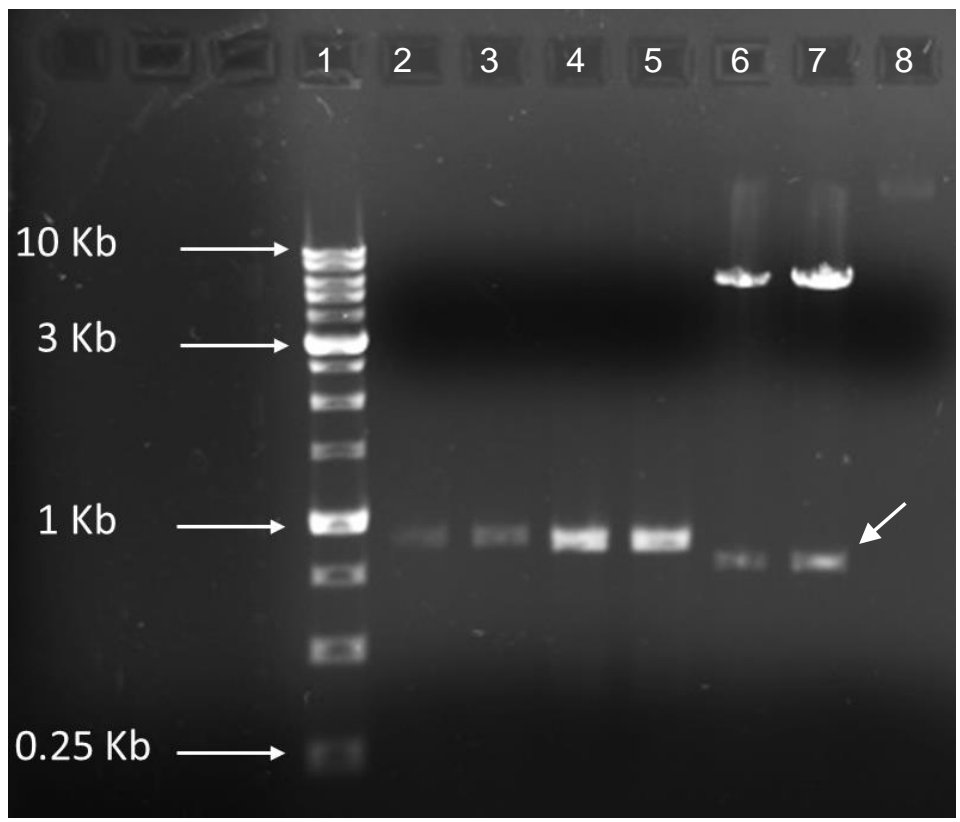

Figure 12: Restriction digestion with $K p n I$ and $X h o I$. Lane 1, 4 $\mu$ L ladder. UP BD (TTHERM_00729230) PCR products digestion ( 1kb): lane 2, undigested UP PCR product; lane 3; digested UP PCR product. UP ISWI1 (TTHERM_00388250) PCR products digestion ( 1 kb): lane 4, undigested UP PCR product; lane 5; digested UP PCR product. There is no difference between the undigested and the digested PCR product because the eliminated flanking DNA sequence at both ends is of approximately 20 base pairs in total. pBKS-FZZ plasmids (with Asf1 cloned in) digestion: lane 6 and 7, two digested plasmids and the released insert (showed with arrow) contained in the plasmid ( 800bp); lane 8, undigested plasmid (digestion control).

\subsection{DNA ligation and transformation}

Prior to ligation and to verify recovery, $2 \mu \mathrm{L}$ of both digested and cleaned PCR products and plasmid (vector) were assessed by electrophoresis. The obtained image of the gel (Figure 13) shows that the fragment recovery was successful since presents the PCR and digested products. 


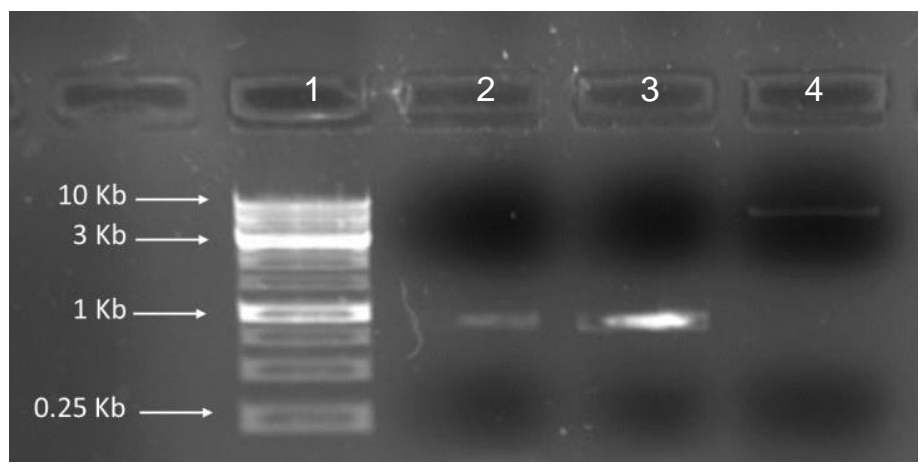

Figure 13: Fragments recovery.

Lane 1, 4 $\mu$ L ladder; Lane 2, UP BD (TTHERM_00729230) PCR products digested and cleaned; lane 3, UP ISWI1 (TTHERM_00388250) PCR products digested and cleaned; lane 4, pBKSFZZ plasmids digested and cleaned.

I ligated the UP BD to the vector (experiment 1) and the UP ISWI1 to the vector (experiment 2) using T4 ligase. I followed the ligation with transformation of competent E.coli (DH5 $)$. From the transformants colonies I selected six colonies from each experiment (1 and 2), growth them in liquid media and isolate the plasmids. From the plasmid isolation, $2 \mu \mathrm{L}$ of each of the twelve plasmid isolations, six corresponding to experiment 1 and six to experiment 2 , were assessed by electrophoresis. The obtained image of the gel (Figure 14) shows that the selected colonies contain a plasmid.

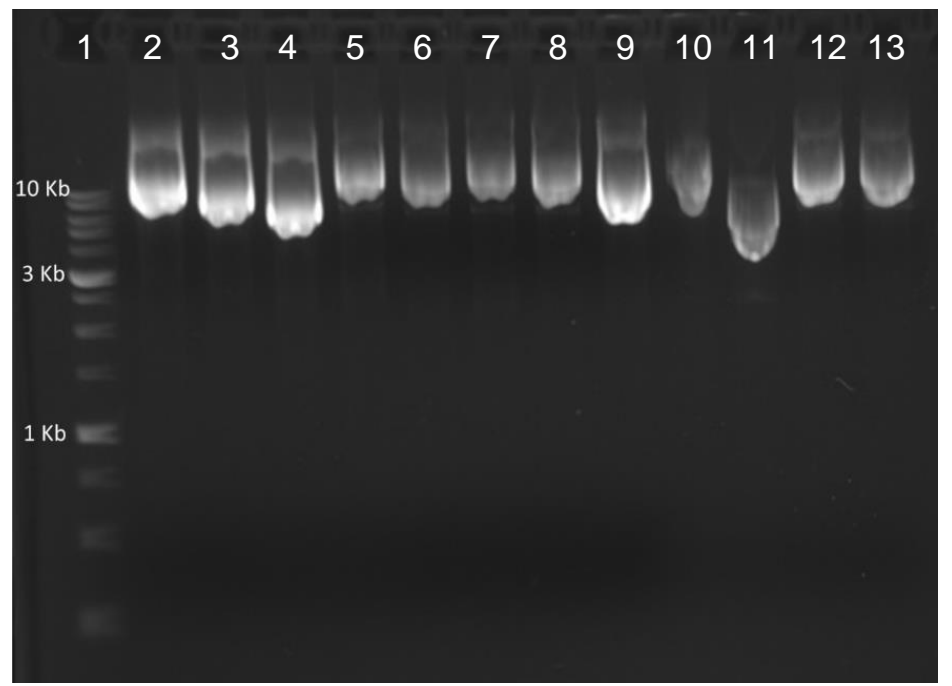

Figure 14: pBKS-FZZ Plasmids after 1kb UP transformation.

Lane 1, 4 $\mu$ L ladder; Lane 2-7, digested UP BD (TTHERM_00729230) PCR products ligated to digested pBKS-FZZ linear plasmids; lane 8-13, digested UP ISWI1 (TTHERM_00388250) PCR products ligated to digested pBKS-FZZ linear plasmids. 
In order to assess if the transformed plasmids contain the approximately $1 \mathrm{~kb}$ in size corresponding to the UP BD and UP ISWI1, I performed double digestion with KpnI and XhoI. To assess if the released portions correspond to the expected size of approximately $1 \mathrm{~kb}$, the digestion products were run in an electrophoresis gel. The obtained image of the gel (Figure 15) shows that the plasmids contain a product of approximately $1 \mathrm{~kb}$.

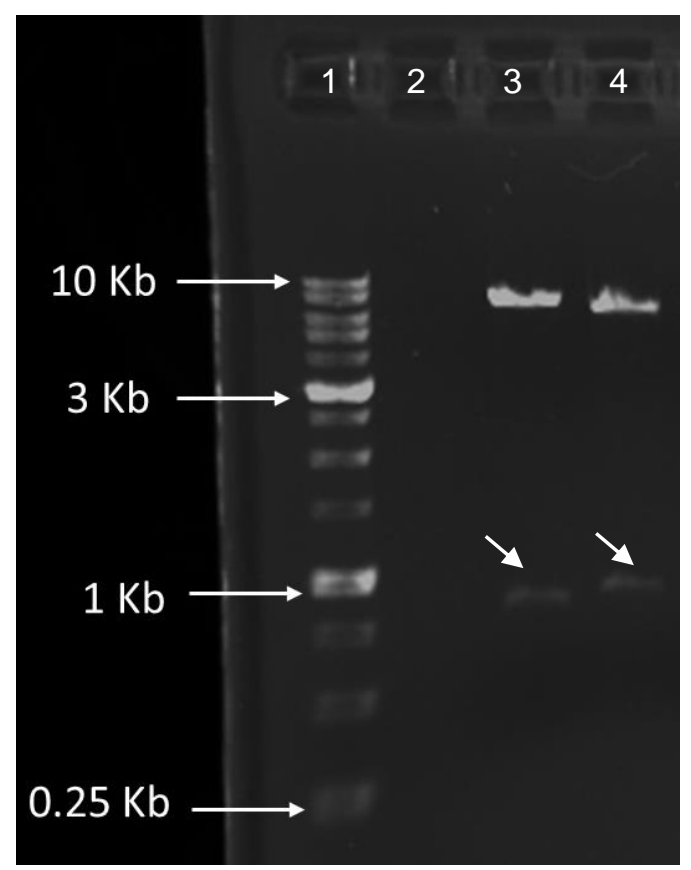

\section{Figure 15: pBKS-FZZ Plasmids digestion.}

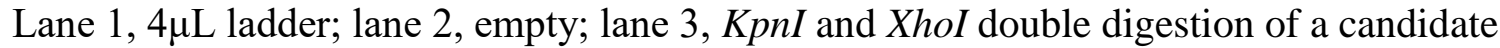
plasmid that may contain the 1kbUP ISWI1 (TTHERM_00388250); lane 4, KpnI and XhoI double digestion of a candidate plasmid that may contain the 1kbUP BD (TTHERM_00729230). The arrows show the approximately $1 \mathrm{~kb}$ sequences.

After Sanger sequencing, the obtained sequence was aligned (Nucleotide EMBOSS Needle, EMBL-EBI) to the gene sequence obtained from ciliate.org, both sequences corresponding to the 1kbUP stream of the stop codon from Iswi1 (TTHERM_00388250) and BD (TTHERM_00729230) were $100 \%$ identical. The previously stored cells at $4{ }^{0} \mathrm{C}$ corresponding to this experiment were grown for plasmid isolation for the subsequent cloning of the $1 \mathrm{kbDOWN}$ stream sequence. The addition of this sequences was successful (data not shown). 


\subsection{Tagging construct recovery}

In order to transform Tetrahymena by biolistics, it is necessary to release the entire tagging cassette from the plasmid backbone to free the ends of the homologous sequence. Double digestion was with KpnI and SacI. Per gene, I used minipreps to extract plasmids from a total of $30 \mathrm{~mL}$ of grown cells obtaining 50ug of miniprep DNA. I double digested the miniprep DNA in 5 reactions per gene. To assess the digestion, I used $2 \mu \mathrm{L}$ of each digestion for electrophoresis. The obtained image of the gel (Figure 16) shows that the digested products contain a product of approximately $3 \mathrm{~kb}$ corresponding to the tagging construct (TC) and .a higher band of approximately $6 \mathrm{~kb}$ corresponding to the plasmid backbone (PB).

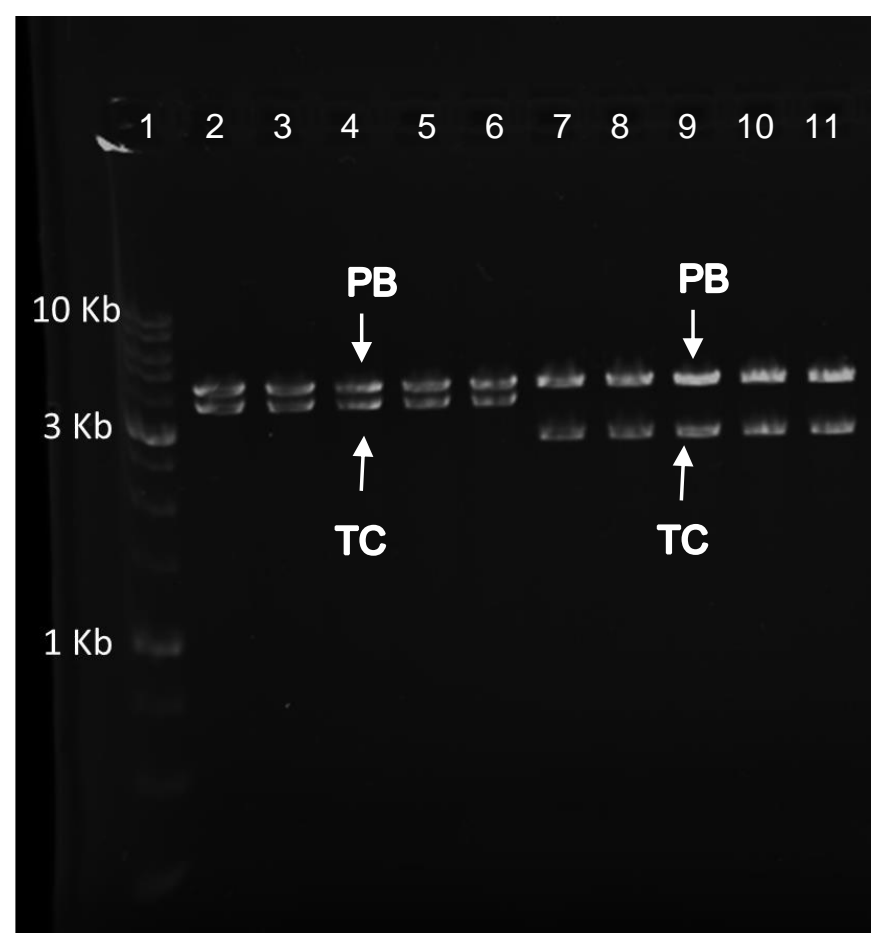

Figure 16: Double digestion to obtain the tagging constructs.

Lane 1, 4 $\mu$ L ladder; lane 2-6, BD (TTHERM_00729230) double digested with KpnI and SacI; lane 7-11, ISW1 (TTHERM_00388250) double digested with KpnI and SacI. The $\sim 3 \mathrm{~kb}$ bands correspond to the tagging constructs (TC) and the higher band of $\sim 6 \mathrm{~kb}$ correspond to the plasmid backbone (PB).

Each of the 5 digestions per gene were cleaned using phenol-chloroform extraction and recovered by ethanol precipitation. To assess if the extraction was successful the DNA was run 
by electrophoresis. The obtained image of the gel (Figure 17) shows that both extractions were successful and contain the tagging construct and plasmid backbone.

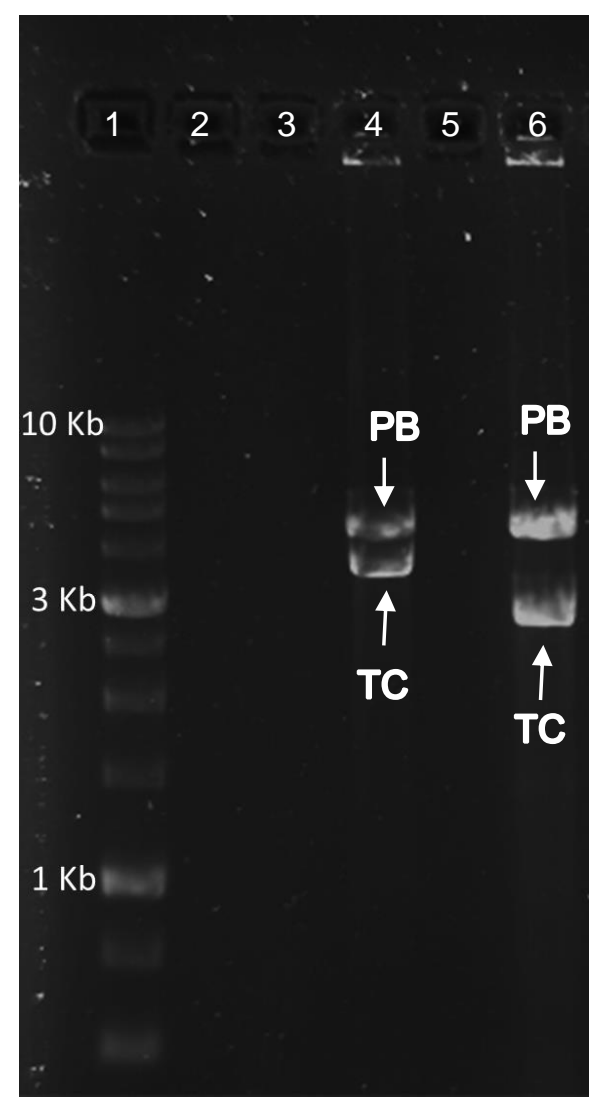

\section{Figure 17: Tagging construct clean up}

Lane 1, 4 $\mu$ L ladder; lane 2-3, empty; lane 4 BD (TTHERM_00729230) double digested with KpnI and SacI and cleaned with phenol:chloroform; lane 5, empty; lane 6, ISWI1 (TTHERM_00388250) double digested with KpnI and SacI and cleaned with phenol:chloroform. The $\sim 3 \mathrm{~kb}$ bands correspond to the tagging constructs (TC) and the higher band of $\sim 6 \mathrm{~kb}$ correspond to the plasmid backbone (PB). 


\section{CHAPTER 2 - Identification of Tetrahymena SWI/SNF and identification and analysis of Ibd1}

In multi-cellular eukaryotes, SWI/SNF complex is known to function as an ATP dependent chromatin remodeler (Vignali et al., 2000). The identity of individual SWI/SNF proteins are well known in fungi (Peterson, Dingwall, \& Scott, 1994)' (Logie \& Peterson, 1999), humans (Muchardt et al., 1996) and Drosophila (Dingwall et al., 1995). In addition to physical interactions within the complex, interactions exists with other proteins that presumable recruit SWI/SNF to specific locations. In humans, $h B R G$ is involved in many protein-protein interactions such as in $\beta$-catenin (Barker et al., 2001), the Fanconi anemia protein A (FANCA) (Otsuki et al., 2001), and Hpl $\alpha$ (Nielsen et al., 2002). Although the putative catalytic subunit Brg1 has been clones and functionally analyze (Fillingham et al., 2006), the SWI/SNF complex remains uncharacterized at the experimental level in T.thermophila.

\subsection{Assessment to determine if Snf5-FZZ pm-r transformants are expressing the tagged protein (Snf5-FZZ) at the expected size}

I started my work with a potential SNF5-FZZ that had been generated previously but not confirmed by western blotting. To confirm the identity of the strains, I used TCA precipitation followed by Western blotting analysis to assess if the provided SNF5-FZZ strains were expressing the tagged protein. When I blotted with the M2 antibody, Figure 18 shows that only the SNF5-FZZ and not the untagged WT strain is expressing a protein of about $60 \mathrm{kDa}$ which corresponds to $18 \mathrm{kDa}$ from the FZZ and $42 \mathrm{kDa}$ size predicted for the Snf5 protein. For the Brg1 antibody, Figure 18 shows that both, a WT strain and the SNF5-FZZ strain, are expressing Brg1 (loading control). This confirms that I have the correct strain.

\subsection{Expression analysis of Snf5}

In order to identify whether Snf5 is member of a Tetrahymena SWI/SNF complex, I affinity purified the FZZ-tagged protein and any co-purifying proteins by performing a one-step 
affinity chromatography on whole cell extracts made from cells in vegetative growth expressing SNF5-FZZ. My Western blotting using M2 antibody for the input and affinity purified material in Figure 18 shows that only the SNF5-FZZ strain is expressing a protein of about 60kDa corresponding to SNF5-FZZ. If Snf5 is a member of Tetrahymena SWI/SNF, then as predicted, it should co-purify with Brg1, the Tetrahymena Snf2 homolog and likely ATP-dependent engine of the complex. Since I had an antibody that recognizes Brg1 (Fillingham et al., 2006), I used it in western blotting. Anti-Brg1 for the input and affinity purified material shows that both the WT and the SNF5-FZZ show a similar layout to the TCA extraction (Figure 18), as expected. However, some degradation of Brg1 was observed (Figure 18). Specifically, after the TCA extraction, Brg1 presented 1 band (Figure 18), and after the whole cell extraction with the gentler AP-MS lysis buffer, Brg1 presented 2-3 bands (Figure 18). If a PTM had been present on Brg1, two bands would have been visible after the TCA extraction. However, this statement is not all inclusive since all Brg1 could be modified with this hypothetical PTM but this is unlikely to happen. Brg1 with a PTM such as phosphorylation or ubiquitination would appear heavier than the WT size and degradation would make it smaller than WT size. Because the additional bands seen are smaller than the WT size I think it is more likely that the multiple bands recognized by anti-Brg1 seen in Fig 17 in the AP experiment represent degradation of Brg1 and not the presence of a PTM.

When blotting with the M2 antibody, Figure 18 shows that only the SNF5-FZZ strain is expressing a protein of about 60kDa corresponding to SNF5-FZZ. WT represents the mock IP. When I blotted with the anti- Brg1 antibody Figure 18 shows that only the SNF5-FZZ is copurifying with Brg1. This is a predicted result based on the fact that Snf5 and Brg1 interact in other systems. Thus as I predicted I have likely purified a Tetrahymena specific SWI/SNF complex. From this result, I expect to see Brg1 identified in the SAINTexpress analysis of the affinity purified material (see next section). 


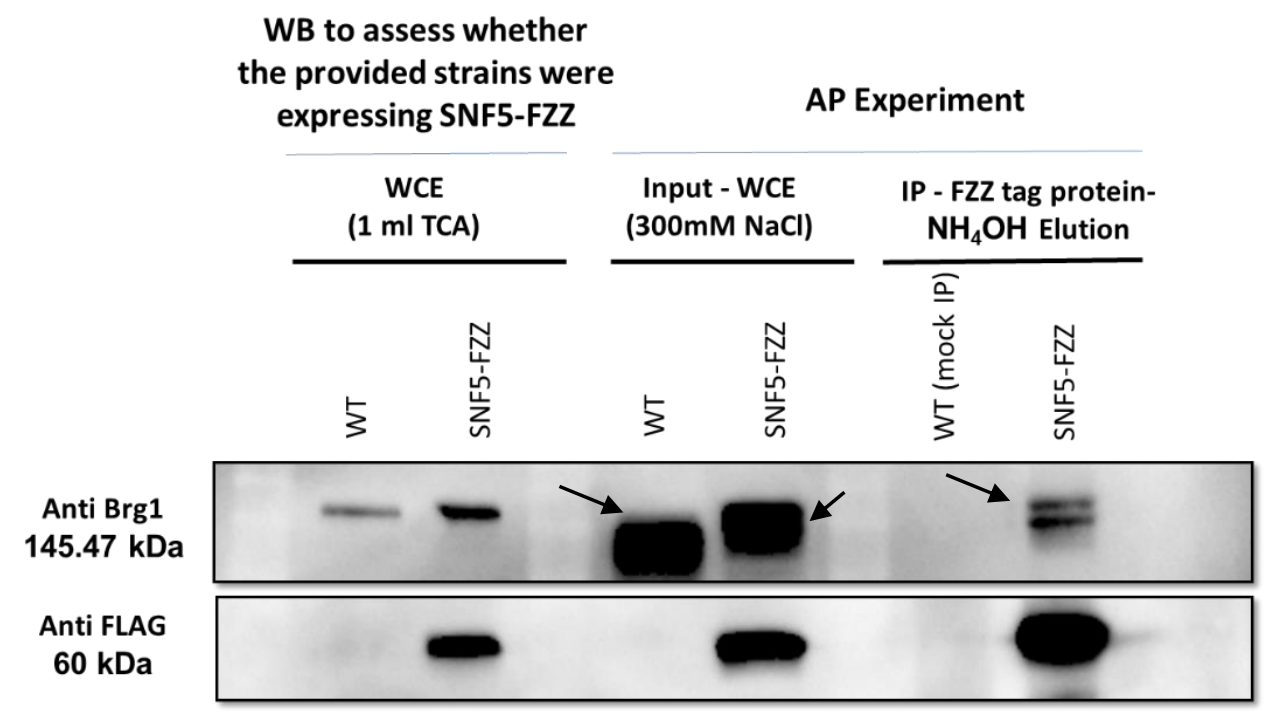

Figure 18. Expression Analysis / Affinity Purification of SNF5-FZZ

Snf5-FZZ is recognized by anti FLAG. The arrows point to where degradation of Brg1 was observed.

\subsection{MS and SAINTexpress analysis of affinity purified Snf5-FZZ during vegetative growth}

With affinity purified SNf5-FZZ we next used LCMS to identify any co-purifying proteins. The approach for this step is to use the affinity purified Snf5-FZZ eluates from the affinity purification step and analyze them using LCMS (AP-MS) to identify protein-protein interaction profiles. The SAINTexpress (Significance Analysis of INTeractome express) algorithm, developed by the Choi, Nesvizhskii and Gingras laboratories in Toronto, Ontario, was used to transform spectral counts obtained for a given bait-prey interaction into the probability of whether the physical interaction is significant or not (Skarra et al., 2011). Therefore only true interactions that pass a set cut-off value were included in a SAINTexpress curated data table (Table 4). At this final step I found the SNF5-FZZ co-purifying proteins, or, the proteins that are interacting with SNF5-FZZ. In order to eliminate background data and obtain a reproducible set of proteins that co-purify with a given bait, these analyses were performed in conjunction with a mock IP from untagged WT cells and database mock IP. Mock IP are very important for SAINTexpress to work properly because with respect to background proteins there are some of these proteins that show up all the time in every mock IP and there are some background proteins that show up only a percentage of the times. Therefore having multiple mock IPs give 
SAINTexpress better confidence when predicting whether a given bait-prey interaction is significant. Similarly, multiple repeats of the given AP-MS is important. AP-MS was repeated four times for each bait to have a rigorous idea of the set of co-purifying proteins. These proteins can be visualized in Table 4 .

The SAINTexpress analysis of the MS data for the four replicates of SNF5-FZZ (Table 4) reveals five of the predicted SWI/SNF members by Fillingham et al, 2006 (Fillingham et al., 2006); Brg1 (also as expected from my western blotting of Figure 18), Swi1, Swi3, and Snf12 in addition to the bait protein Snf5 (Table 1) as well as 8 potentially novel members of Tetrahymena SWI/SNF; one protein that contains a PHD domain (TTHERM_00241840), tetrin, four Tet-specific proteins, one ciliate-specific and interestingly one that contains a BD. PHD domain containing proteins are not present in yeast or human SWI/SNF complexes. One function of PHD domains is to mediate specific interactions with methylated lysine on histone proteins to positively regulate transcription (Shi et al., 2006). In this specific case the found PHD domain is conserved with that of yeast JHD2 which is a HDMT (Table 4). Four Tet-specific proteins with no predicted functions were found and they may be acting as species-specific members of the SWI/SNF complex in Tetrahymena thermophila. A ciliate-specific protein (TTHERM_00092790) was found with no predicted function and it may be a conserved member of SWI/SNF complex in ciliates. Interestingly, tetrin (TTHERM_00006320) is an insoluble cytoskeletal protein unique to cilia (Williams \& Hontst, 1995) and was present in our soluble extraction. Another interesting result is that a bromodomain-containing protein (TTHERM_00729230) co-purifies with Snf5-FZZ. Recall that an earlier functional analysis of Tetrahymena Brg1 (Fillingham et al. 2006) (Fillingham et al., 2006) revealed that unlike yeast and human version it does not possess a C-terminal bromodomain. The presence of the BD containing protein is consistent with a SWI/SNF complex in Tetrahymena interacting with acetylated histones similar to SWI/SNF in yeast and human cells. Next I decided to perform a reciprocal purification with a member of Table 4 with the intent to confirm the identity of members of the SWI/SNF complex in Tetrahymena

Of particular interest was the BD-containing protein, TTHERM_00729230. As previously mentioned, bromodomains mediate specific physical interaction with acetyl-lysine on 
histones (Dhalluin et al., 1999). In most eukaryotes the catalytic subunit of SWI/SNF itself possesses a bromodomain (Tang et al., 2010). The catalytic subunit in Tetrahymena BRG1/SNF2 is unusual in that it does not possess a bromodomain (Fillingham et al., 2006).

Table 4: SAINTexpress-curated MS data for 4 replicates of SNF5-FZZ affinity purification during vegetative growth.

MS data for SNF5-FZZ reveals predicted and novel members of the putative Tetrahymena SWI/SNF complex. BOLD: Predicted in Fillingham et al., 2006 (Fillingham et al., 2006). Italic: Novel. Only true interactions that pass a set high cut-off value were included in this table. SpecSum represents the abundance of each protein.

\begin{tabular}{|l|l|l|l|}
\hline \multicolumn{1}{|c|}{ Protein } & \multicolumn{1}{c|}{ Gene Name } & \multicolumn{1}{c|}{ SpecSum } & \multicolumn{1}{c|}{ Yeast } \\
\hline Swi3 & TTHERM_00584840 & $\mathbf{3 3 8}$ & Swi3 \\
\hline Tet-specific & TTHERM_00346460 & 284 & --- \\
\hline Brg1 & TTHERM_01245640 & $\mathbf{2 5 6}$ & Snf2/Sth1 \\
\hline Swi1 & TTHERM_00243900 & $\mathbf{1 5 4}$ & Swi1 \\
\hline Snf5 (BAIT) & TTHERM_00304150 & $\mathbf{1 4 3}$ & Snf5 \\
\hline Tet-specific & TTHERM_00129650 & 138 & --- \\
\hline PHD domain & TTHERM_00241840 & 120 & JHD2 \\
\hline Snf12 & TTHERM_00925560 & $\mathbf{7 8}$ & Snf12 \\
\hline tetrin & TTHERM_00006320 & 76 & --- \\
\hline ciliate-specific & TTHERM_00092790 & 70 & --- \\
\hline Tet-specific & TTHERM_00657560 & 56 & --- \\
\hline Tet-specific & TTHERM_00637690 & 43 & --- \\
\hline BD protein & TTHERM_00729230 & 40 & --- \\
\hline
\end{tabular}

\subsection{Molecular cloning and generation of FZZ tagged TTHERM_00729230 (Ibd1)}

I therefore generated transformants of Tetrahymena expressing FZZ-tagged BD-containing protein (TTHERM_00729230) from its WT chromosomal locus. The Tetrahymena BD protein was chosen for epitope tagging since it is conserved with the Sth1 bromodomain in yeast with a low BLASTP E-value of $3 \mathrm{E}^{-5}$. This E-value suggests homology between the Sth1 bromodomain and the bromodomain of the BD protein. I used the molecular cloning and transformation methods described in chapter 1 to FZZ tag the BD protein (TTHERM_00729230) in order to 
confirm reciprocal AP-MS interactions with SNF5. The rationale behind reciprocal purifications is to validate the identity of the SWI/SNF complex through co-purification via an additional subunit than Snf5, and possibly identify new co-purifying proteins. A Snf5-FZZ co-purifying protein, the bromodomain protein, was selected for FZZ tagging because of our interest in the lost bromodomain of Brg1 as mentioned above. Using the described molecular cloning methods I obtained 5 paromomycin resistant (pm-r) transformants each of which should express FZZ tagged TTHERM_00729230

\subsection{Assessment to determine if pm-r transformants are expressing the tagged protein (BD-FZZ) at the expected size}

I used TCA precipitation to generate a whole cell extract which I separated on SDSPAGE and used Western blotting analysis to assess whether the 5 transformed pm-r cells are expressing BD-FZZ. Of the 5 pm-r transformants, three were independent transformants in B2086 strain; BD-FZZ B1, BD-FZZ B2, and BD-FZZ B3, and two from CU428 cells; BD-FZZ $\mathrm{C} 1$ and BD-FZZ C2. Western blotting of whole cell extracts using the M2 antibody shows that

only the SNF5-FZZ strain is expressing a protein of about $50 \mathrm{kDa}$ which corresponds to $18 \mathrm{kDa}$ from the FZZ and $32 \mathrm{kDa}$ from the BD protein (Figure 19). Western blotting using the Brg1 antibody shows that both the WT strain and the BD-FZZ strains are expressing Brg1 (Figure 19, loading control). Therefore, I successfully engineered two new BD-FZZ strains with two different mating types.

\subsection{Analysis of Ibd1 interactions during vegetative growth}

In order to determine the expression pattern of BD-FZZ in the Tetrahymena life cycle I affinity purified Snf5-FZZ along with an y co-purifying proteins by performing one-step affinity chromatography on whole cell extracts made from cells in vegetative growth expressing BD-FZZ (I used BD-FZZ B3 from (Figure 19).

Western blotting using the Brg1 antibody (Figure 19) shows that both the WT and the BD-FZZ have Brg1; however, like my earlier experiments, degradation of this protein is present 
(showed with arrows in Figure 19). Western blotting using the M2 antibody shows that only the BD-FZZ strain is expressing a protein of about $50 \mathrm{kDa}$ corresponding to BD-FZZ, as expected (Figure 19). WT represents the mock IP from untagged cells. Western blotting using the Brg1 antibody shows that the BD-FZZ but not the mock IP from untagged cells is co-purifying with Brg1, consistent with the BD-containing protein being a member of Tetrahymena SWI/SNF as expected from my Snf5 analysis (Table 1). Degradation of Brg1 is present on the western blot (showed with arrows in Figure 19) as before in Figure 18. From this data I can assume that the BD protein and Brg1 are bona fide members of the SWI/SNF complex and I expect to see Brg1 in SAINTexpress analysis of the affinity purified material (see next section).

\begin{tabular}{c} 
WB to assess whether pm-r cells are \\
expressing BD-FZZ \\
\hline $\begin{array}{c}\text { WCE } \\
(1 \mathrm{ml} \mathrm{TCA})\end{array}$
\end{tabular}

\begin{tabular}{|c|c|}
\hline AP & eriment \\
\hline $\begin{array}{c}\text { Input - WCE } \\
(300 \mathrm{mM} \mathrm{NaCl})\end{array}$ & $\begin{array}{l}\text { IP - FZZ tag protein- } \\
\mathrm{NH}_{4} \mathrm{OH} \text { Elution }\end{array}$ \\
\hline
\end{tabular}

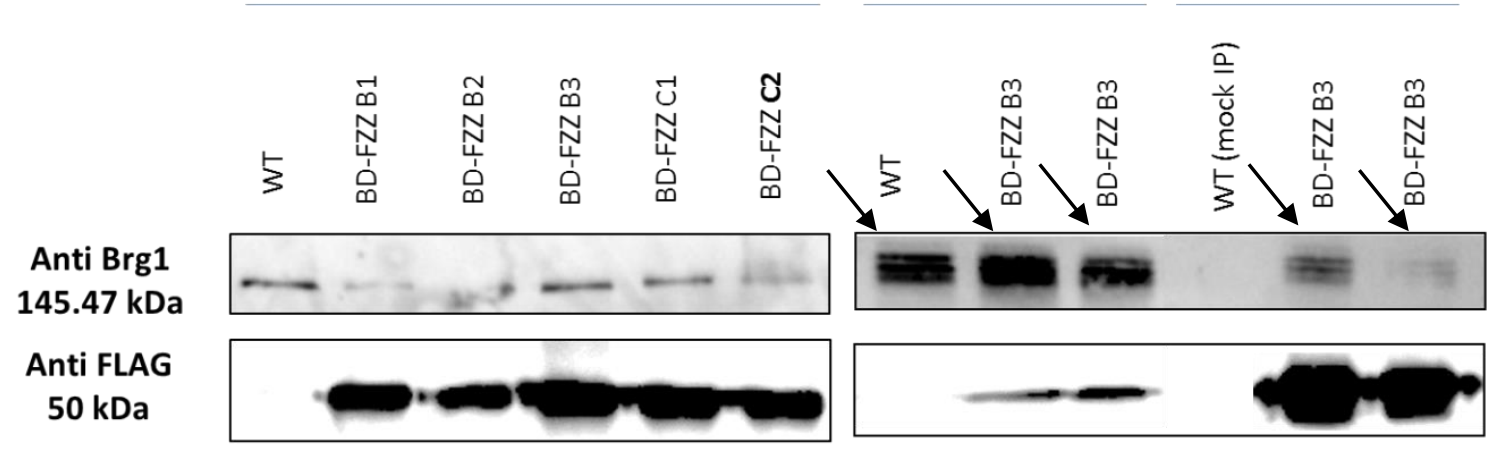

Figure 19: Expression analysis of BD-FZZ during vegetative growth

The BD containing protein is recognized by anti FLAG and co-purifies with Brg1. The arrows point to where degradation of Brg1 was observed. WT represents the mock IP from untagged cells.

\subsection{SAINTexpress analysis of MS data from affinity purified BD-FZZ during vegetative growth}

The SAINTexpress analysis of MS data from the affinity purified BD protein is shown in Table 5. This data suggests that BD protein is a bona-fide subunit of the SWI/SNF complex, in addition to other chromatin remodeling complexes, such as, a putative Tetrahymena SWR and SAGA and a HMT (Table 5, and see below). For this reason I renamed the BD protein 
Interactive Bromodomain Protein 1 (Ibd1) since it interacts with multiple transcription associated complexes. From the SAINTexpress curated MS data, I also found different types of actin. Actin related proteins are found in several ATP dependent chromatin remodeling complexes, although their precise function is unclear (Schubert et al., 2013). The presence of actin during this purification process suggests that the co-purifying subunits are related to chromatin remodeling.

Table 5: SAINTexpress-curated data for 3 replicates of BD-FZZ during vegetative growth The SpecSum represents the abundance of each protein. The found proteins were labeled as follows (SWI/SNF-C), (Putative SWR-C), (Putative HMT), (Putative SAGA-C), and (undetermined) based on their predicted function.

\begin{tabular}{|l|l|l|}
\hline \multicolumn{1}{|c|}{ Protein } & \multicolumn{1}{c|}{ Gene Name } & SpecSum \\
\hline BD protein (BAIT) & TTHERM_00729230 & 1348 \\
\hline Swi3 (SWI/SNF-C) & TTHERM_00584840 & 495 \\
\hline RvB2 (Putative SWR-C) & TTHERM_00046920 & 372 \\
\hline RvB1 (Putative SWR-C) & TTHERM_00476820 & 216 \\
\hline Brg1 (SWI/SNF-C) & TTHERM_01245640 & 176 \\
\hline Atxr3/Set1 (Putative HMT) & TTHERM_00486440 & 154 \\
\hline Swr1 (Putative SWR-C) & TTHERM_01546860 & 134 \\
\hline Actin (Putative SWR-C) & TTHERM_00975380 & 114 \\
\hline Snf12 (SWI/SNF-C) & TTHERM_00925560 & 90 \\
\hline Swi1 (SWI/SNF-C) & TTHERM_00243900 & 80 \\
\hline PHD finger (undetermined) & TTHERM_00444700 & 67 \\
\hline Actin (Putative SWR-C) & TTHERM_00317000 & 52 \\
\hline Snf5 (SWI/SNF-C) & TTHERM_00304150 & 46 \\
\hline Arp6 (Putative SWR-C) & TTHERM_01005190 & 45 \\
\hline ciliate-specific (SWI/SNF-C) & TTHERM_00092790 & 44 \\
\hline Gcn5 (Putative SAGA-C) & TTHERM_00248390 & 44 \\
\hline Hypothetical protein (undetermined) & TTHERM_00170260 & 34 \\
\hline Tet-specific (SWI/SNF-C) & TTHERM_00346460 & 33 \\
\hline Swc4 (Putative SWR-C) & TTHERM_00357110 & 33 \\
\hline & & \\
\hline
\end{tabular}




\begin{tabular}{|l|l|l|}
\hline ciliate-specific (undetermined) & TTHERM_00136450 & 29 \\
\hline PHD domain (SWI/SNF-C) & TTHERM_00241840 & 29 \\
\hline Tet-specific (SWI/SNF-C) & TTHERM_00637690 & 27 \\
\hline AT-hook containing (undetermined) & TTHERM_00355040 & 23 \\
\hline Tet-specific (SWI/SNF-C) & TTHERM_00129650 & 22 \\
\hline Ada2 (Putative SAGA-C) & TTHERM_00790730 & 21 \\
\hline Swc2-like (Putative SWR-C) & TTHERM_00388500 & 18 \\
\hline Yaf9 (Putative SWR-C) & TTHERM_00561450 & 13 \\
\hline Hypothetical protein (undetermined) & TTHERM_00046150 & 4 \\
\hline
\end{tabular}

\subsection{The first ciliate SWI/SNF complex}

Through AP-MS of Tetrahymena Snf5, and Snf5-interacting Ibd1, I have identified the first protist SWI/SNF complex, based on the intersection of the 2 sets of co-purifying proteins (Refer to Figure 20 for Venn diagram intersections). The Tetrahymena thermophila SWI/SNF complex contains 11 subunits (Table 6). It has a catalytic subunit (Brg1) and a standalone bromodomain containing protein (Ibd1). This SWI/SNF complex also has well characterized orthologues subunits present in yeast (Swi1, Swi3, Snf5, and Snf12), a ciliate specific protein (TTHERM_00092790), 3 Tetrahymena specific proteins (TTHERM_00346460, TTHERM_00129650 and TTHERM_00637690) and a PHD domain containing protein (TTHERM_00241840). PHD domain containing proteins are not known members of the SWI/SNF complex in other organisms. One function of PHD domains is to mediate specific interactions with methylated lysine on histone proteins to positively regulate transcription (Shi et al., 2006). In this specific case the found PHD domain is conserved with the yeast JHD2 (HDMT). In conclusion, perhaps Tetrahymena SWI/SNF has two proteins that recognize PTM on histones, the PHD domain and Ibd1. These two proteins could work in conjunction to positively regulate transcription at an increased rate. 
Table 6: Tetrahymena thermophila SWI/SNF complex

\begin{tabular}{|l|l|l|}
\hline \multicolumn{1}{|c|}{ Protein } & \multicolumn{1}{c|}{ Gene } & \multicolumn{1}{c|}{ Yeast } \\
\hline Brg1 (catalytic subunit) & TTHERM_01245640 & Snf2/Sth1 \\
\hline Ibd1 (Interactive Bromodomain Protein 1) & TTHERM_00729230 & Sth1 bromodomain \\
\hline Snf5 & TTHERM_00304150 & Snf5 \\
\hline Swi3 & TTHERM_00584840 & Swi3 \\
\hline Swi1 & TTHERM_00243900 & Swi1 \\
\hline Snf12 & TTHERM_00925560 & Snf12 \\
\hline Ciliate-specific & TTHERM_00092790 & --- \\
\hline Tet-specific & TTHERM_00346460 & --- \\
\hline Tet-specific & TTHERM_00129650 & --- \\
\hline Tet-specific & TTHERM_00637690 & --- \\
\hline PHD domain & TTHERM_00241840 & JHD2 \\
\hline
\end{tabular}

\subsection{Putative SWR and SAGA complexes}

In addition to the SWI/SNF complex and from data from Table 5, I am beginning to establish members of Tetrahymena thermophila putative SWR (Table 7) and SAGA (Table 8) complexes.

Table 7. Tetrahymena thermophila putative SWR complex

\begin{tabular}{|l|l|}
\hline \multicolumn{1}{|c|}{ Protein } & \multicolumn{1}{c|}{ Gene } \\
\hline RvB2 & TTHERM_00046920 \\
\hline Ibd1 & TTHERM_00729230 \\
\hline RvB1 & TTHERM_00476820 \\
\hline Swr1 & TTHERM_01546860 \\
\hline Actin & TTHERM_00975380 \\
\hline Actin & TTHERM_00317000 \\
\hline Arp6 & TTHERM_01005190 \\
\hline Swc4 & TTHERM_00357110 \\
\hline Swc2-like & TTHERM_00388500 \\
\hline Yaf9 & TTHERM_00561450 \\
\hline
\end{tabular}


Table 8. Tetrahymena thermophila putative SAGA complex

\begin{tabular}{|l|l|}
\hline \multicolumn{1}{|c|}{ Protein } & \multicolumn{1}{c|}{ Gene } \\
\hline Gcn5 & TTHERM_00248390 \\
\hline Ibd1 & TTHERM_00729230 \\
\hline Ada2 & TTHERM_00790730 \\
\hline
\end{tabular}

\subsection{During vegetative growth Ibd1 interacts with members of chromatin remodeling complexes related to transcription}

From the data obtained from the SAINTexpress curated MS analysis of Ibd1-FZZ AP, individual proteins were identified and assigned to a protein complex (SWR or SAGA) based on orthologue proteins present in other organisms. SWI/SNF proteins identified as explained above in section 4.8. I predicted that $\mathrm{Ibd} 1$ is involved in several independent protein complexes and all these complexes form a mega complex to increase transcription. The Ibd1 interacting proteins were grouped in predicted complexes using Venn diagrams (Figure 20). I grouped the members of each transcription related complex that interacts with Ibd1. The complexes, SWI/SNF, SWR$\mathrm{C}$, and SAGA related subunits and a HMT protein, are present in yeast and may be homologous in Tetrahymena. Ibd1 also interacts with other proteins that were classified in the group "Ibd1 (other interactions)" showed in Figure 20. These proteins are two hypothetical proteins (TTHERM_00046150 and TTHERM_00170260), a PHD Finger (TTHERM_00444700, not the same as SWI/SNF PHD domain; TTHERM_00241840), an AT-hook containing (TTHERM_00355040) and a ciliate-specific (TTHERM_00136450 not the same as SWI/SNF ciliate specific; TTHERM_00092790).

In conclusion, Ibd1 is shared with multiple protein complexes (SAGA, SWR, and SWI/SNF) and possibly a HMT protein all of which share common function in chromatin remodeling and transcription. 


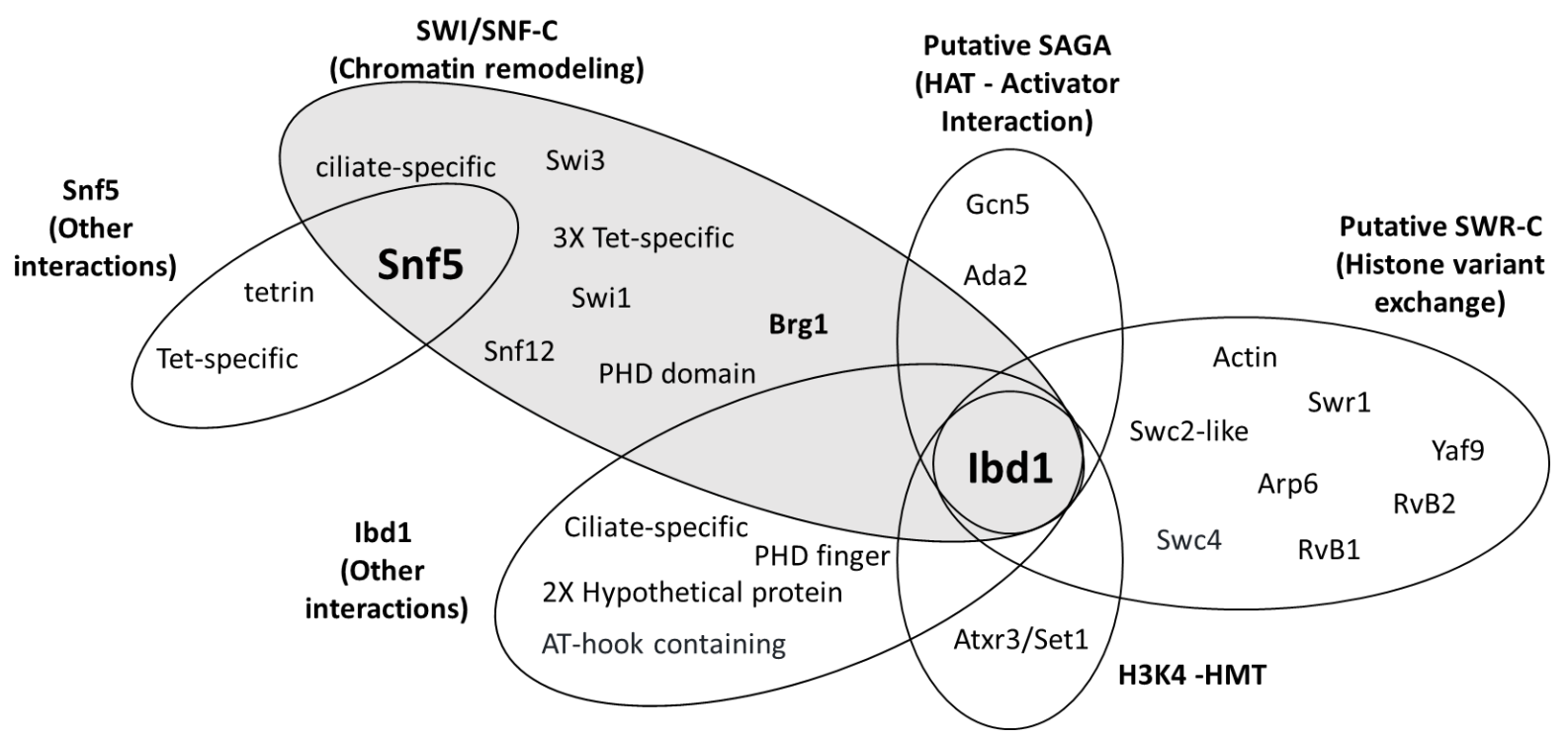

Figure 20: Comprehensive analysis of the Ibd1 interactome during vegetative growth.

The Tetrahymena SWI/SNF-C was found based on the intersection of the two sets of copurifying proteins Snf5 and Ibd1. In addition, Ibd1 interacts with members of other chromatin remodeling complexes, such as, a putative SWR-C and SAGA and a HMT. Ibd1 also interacts with proteins that cannot be grouped in any of the mentioned complexes at this time.

\subsection{Expression analysis of BD-FZZ during T.thermophila's life cycle}

The purpose of this experiment was to corroborate the Ibd1 protein expression profile with the mRNA expression profile publically available from ciliate.org which indicates that at the mRNA level this gene expresses throughout Tetrahymena's life cycle. It also will give us a good reference whether Ibd1 expresses at different hours related to specific key events during conjugation such as vegetative growth, starvation, meiosis $(3 \mathrm{H})$, gametic nuclei fusion $(4.5 \mathrm{H})$, zygotic nuclei duplication $(6 \mathrm{H})$, anlagen development $(8 \mathrm{H})$ and when the daughter cells have formed $(24 \mathrm{H})$.

I used western blotting of TCA-generated whole cell extracts to perform a time course expression analysis of Ibd1. I used Brg1 as a loading control for expression throughout T.thermophila's life cycle, and Pdd1 strictly as a developmental loading control. With these results, it was evident that Ibd1 expresses throughout T.thermophila's life cycle, and it might be stable enough to be present at $24 \mathrm{H}$ after mating when the new MAC is active. 

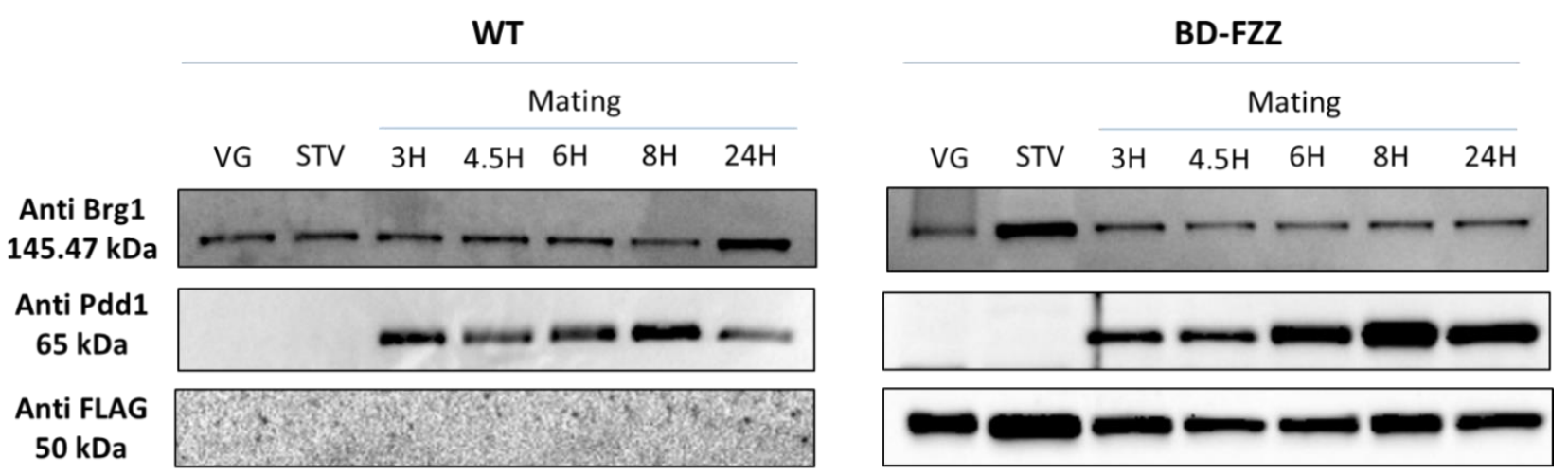

Figure 21: Expression analysis of Ibd1-FZZ during T.thermophila's life cycle Ibd1 (Anti FLAG) expresses throughout T.thermophila's life cycle. Brg1 is a loading control for expression throughout T.thermophila's life cycle and Pdd1 is strictly a developmental loading control.

\subsection{Analysis of Ibd1 interactions during conjugation}

In order to identify the set of proteins that co-purify with Ibd1-FZZ I performed one-step affinity chromatography on whole cell extracts made from cells expressing Ibd1-FZZ (I used Ibd1-FZZ B3 x BD-FZZ C1) and harvested 5 hours after the initiation of conjugation.

Western blotting of affinity purified Ibd1-FZZ using M2 antibody shows that only the BD-FZZ cells mating are expressing a protein of about 50kDa corresponding to BD-FZZ (Figure 22). I used Pdd1p antibody as developmental control and its signal was not present for this blot in the WCE, perhaps because it is not soluble (data not shown). Even though I cannot observe Pdd1p in the WB analysis, I know that the cells are conjugating based on microscope visualization. For the Brg1 antibody Figure 22 shows that both the WT cells mating and the BDFZZ cells mating have degradation of Brg1.

Western blotting of affinity purified material using the M2 antibody shows that only the BD-FZZ cells mating are expressing a protein of about 50kDa corresponding to BD-FZZ (Figure 22). WT represents the mock IP. Western blotting using the Brg1 antibody (Figure 22) shows that BD-FZZ cells mating are not co-purifying with Brg1. This is different than what occurs during vegetative growth (Figure 19). From this result it seems possible that during conjugation 
Ibd1 does not co-purify with Brg1. Perhaps during conjugation Idb1 does not interact with SWI/SNF but remains bound to other complexes such as SWR, SAGA and HMT and possibly new ones. Alternatively, it is possible that during conjugation Brg1 co-purifies with Ibd1 but the amount of it is reduced to a level that is difficult to detect using western blotting. To answer this, next I equilibrated the relative amount of Ibd1 to be loaded in SDS-PAGE from the samples taken during vegetative growth and conjugation (see below). In addition, MS will provide further data (see below).

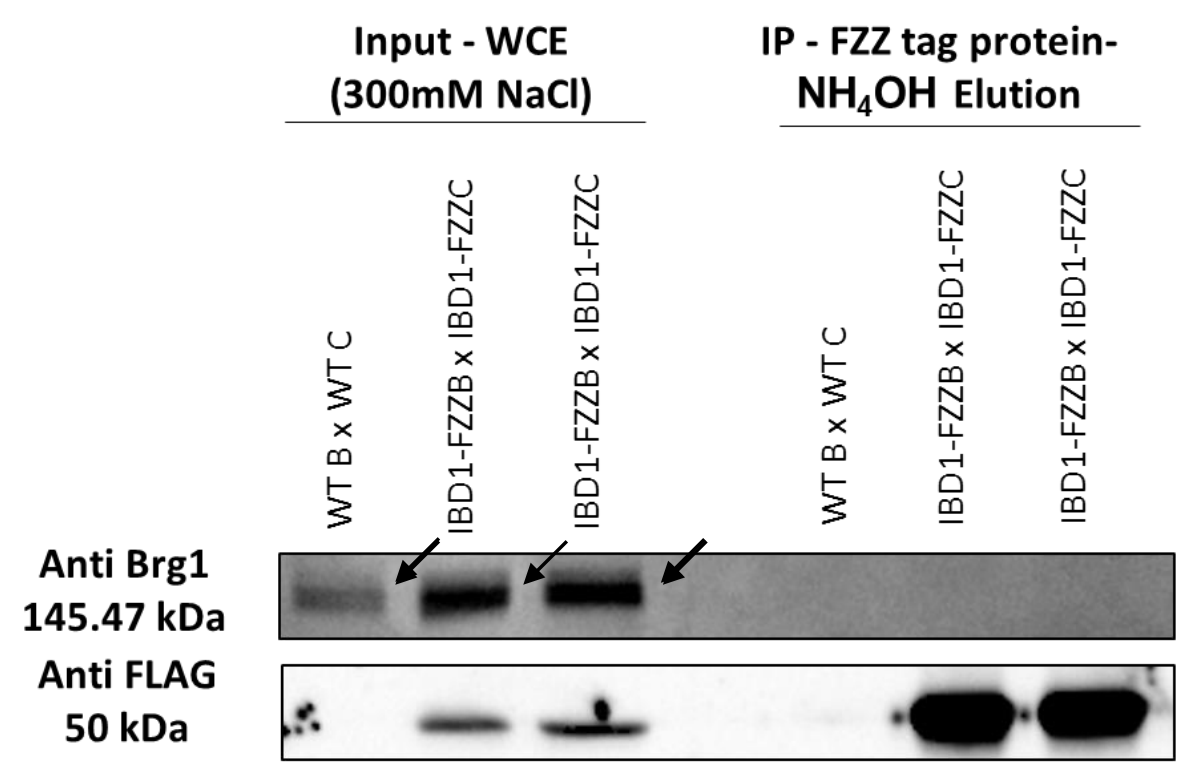

Figure 22: Expression analysis of BD-FZZ 5 hours after conjugation.

In the IP samples taken during conjugation Brg1 cannot be detected. Pdd1p cannot be detected (data not shown). The arrows point to where degradation of Brg1 was observed. Ibd1 is recognized by anti FLAG.

\subsection{SAINTexpress curation of MS data from AP of Ibd1-FZZ during conjugation}

Conversely to WB analysis, SAINTexpress analysis of BD-FZZ cells 5 hours after initiation of mating shows that Ibd1 still interacts with Brg1. Ibd1 interacts with less proteins than during vegetative growth; however, all the interacting proteins are the same than during vegetative growth and there are not new interacting proteins (Table 9). At this stage Ibd1 still interacts with subunits of SWI/SNF, putative SWR-C, HMT and putative SAGA. With this data, 
I can now answer the question, "What is the composition of Ibd1 protein-protein interactions during conjugation?"

Table 9: SAINTexpress-curated data for 2 replicates of BD-FZZ 5 hours after mating.

\begin{tabular}{|l|l|l|}
\hline \multicolumn{1}{|c|}{ Protein } & Gene Name & SpecSum \\
\hline BD protein (BAIT) & TTHERM_00729230 & 738 \\
\hline RvB2 (Putative SWR-C) & TTHERM_00046920 & 263 \\
\hline RvB1 (Putative SWR-C) & TTHERM_00476820 & 170 \\
\hline Swr1 (Putative SWR-C) & TTHERM_01546860 & 137 \\
\hline Actin (Putative SWR-C) & TTHERM_00975380 & 106 \\
\hline Swi3 (SWI/SNF-C) & TTHERM_00584840 & 100 \\
\hline PHD Finger (SWI/SNF-C) & TTHERM_00444700 & 57 \\
\hline Gcn5 (Putative SAGA-C) & TTHERM_00248390 & 41 \\
\hline Arp6 (Putative SWR-C) & TTHERM_01005190 & 41 \\
\hline AT-hook containing (undetermined) & TTHERM_00355040 & 35 \\
\hline Swc4 (Putative SWR-C) & TTHERM_00357110 & 32 \\
\hline ciliate-specific (undetermined) & TTHERM_00136450 & 26 \\
\hline Swc2-like (Putative SWR-C) & TTHERM_00388500 & 22 \\
\hline Atxr3/Set1 (Putative HMT) & TTHERM_00486440 & 22 \\
\hline Ada2 (Putative SAGA-C) & TTHERM_00790730 & 19 \\
\hline Hypothetical protein (undetermined) & TTHERM_00046150 & 16 \\
\hline Snf12 (SWI/SNF-C) & TTHERM_00925560 & 13 \\
\hline Brg1 (SWI/SNF-C) & TTHERM_01245640 & 11 \\
\hline ciliate specific (SWI/SNF-C) & TTHERM_00092790 & 7 \\
\hline Swi1 (SWI/SNF-C) & TTHERM_00243900 & 6 \\
\hline
\end{tabular}




\subsection{Ibd1 modifies its interactions at 5 hours after conjugation and during vegetative growth}

From an inspection of Table 7, Ibd1 still interacts with SWI/SNF, putative SWR-C, HMT and putative SAGA sub-units 5 hours after initiation of conjugation. At this stage Ibd1 interacts with less proteins than during vegetative growth; however, all the interacting proteins are the same than during vegetative growth and there are not new interacting proteins To see if there are any changes in the relative amount of Ibd1 interacting with these respective protein complexes, I set the SpecSum values for the baits (BD-FZZ) in Table 5 and Table 9 to 1 , allowing me to standardize the remaining values in each table in relation to the corresponding bait. I then compared the trends of the two tables in Figure 23 (data analysis in Table 11, Appendix 2) and observed in both that there is a decrease of all SWI/SNF subunits ( $-76.51 \%$ on average); accounting for why Brg1 (-88.58\%) could not be detected in the SDS-PAGE (Figure 24). Another protein that decreases in conjugation in the amount of Ibd1 bound is the HMT ($73.91 \%)$.

Not all proteins decrease: there is an increase of all putative SWR-C (65.67\% on average) and putative SAGA (67.73\% on average) subunits present in this purification. Perhaps Ibd1 has an important role in histone variant exchange and HAT related proteins at 5 hours after initiation of conjugation and not as a member of the SWI/SNF complex or HMT.

Interestingly, this analysis also shows that the proteins that are in the group "Ibd1 (other interactions)" showed in Figure 20 such as hypothetical protein (TTHERM_00046150, not a member of the SWI/SNF complex) drastically increases in percentage bound to Ibd1 (630\%) during conjugation. The protein PHD Finger (TTHERM_00444700, not the same as SWI/SNF PHD domain; TTHERM_00241840), an AT-hook containing (TTHERM_00355040) and a ciliate-specific (TTHERM_00136450 not the same as SWI/SNF ciliate specific; TTHERM_00092790) increase 55.39\%, 177.95\% and 63.76\%, respectively (Figure 23). Perhaps all the proteins that are increasing in amount during conjugation are important for this stage. Calculations are in the Appendix 2. 


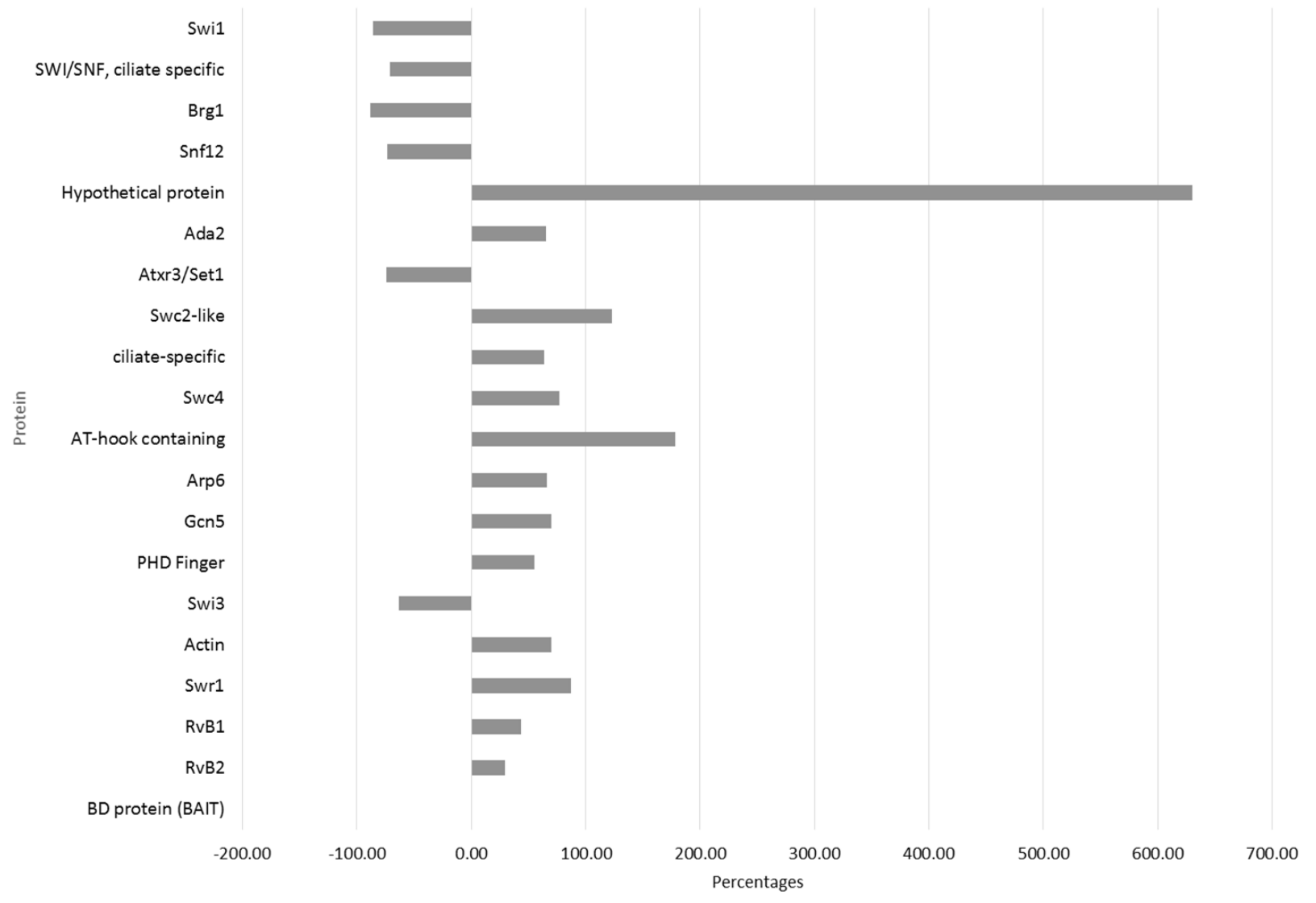

Figure 23: Relative proportion of encountered proteins during vegetative growth and 5 hours after initiation of conjugation

For percentages, if $\%>0$ there is more protein interacting with Ibd1 in conjugation or if $\%<0$ there is less protein interacting with Ibd1 during conjugation compared to during vegetative growth.

\subsection{SDS-PAGE to equilibrate the relative amount of Ibd1}

In Figure 19, we can observe that Ibd1 co-purify with Brg1 during vegetative growth and during conjugation Figure 22 shows that Brg1 does not co-purify with Brg1. I initially hypothesized that at 5 hours after initiation of conjugation there is: 1] a decrease of Brg1 along with a decrease of the SWI/SNF complex subunits; or 2] total absence of the Brg1 subunit alone; or 3] total absence of the SWI/SNF complex. AP-MS showed that there is reduced presence of Brg1 during conjugation compared to vegetative growth, suggesting that during conjugation the SWI/SNF complex decreases in abundance (Figure 23). Thus, probably the decrease of Brg1 influences the sensitivity of WB analysis. We also have to consider that for the previous WB 
shown in Figure 19 and Figure 22 the IP loaded samples were not representing equivalent expression of Ibd1. In order to visualize equivalent expression of Ibd1 with respect to Brg1, it was necessary to increase the amount of IP sample added from conjugation and reduce the amount added from vegetative growth. In conclusion, I found that in IP samples the relative amount of interacting Brg1 to Ibd1 is less, as is shown in Figure 24. This is consistent with the previous result in Figure 23.

\subsection{Pdd1p is insoluble}

As seen in Figure 21, I ran samples belonging to TCA precipitations throughout Tetrahymena thermophila's life cycle. In this WB, Pdd1p can be observed. However, as seen in Figure 22, I ran samples belonging to soluble extracts taken during conjugation. It is evident here that Pdd1p cannot be observed and I hypothesized that Pdd1p is insoluble in our extraction buffer that we use for generation of WCE for AP. I used fresh TCA extracts and compared them to soluble extracts. This can be seen in Figure 24, where anti-Pdd1 recognizes the TCA WCE and not the input WCE. This confirms that Pdd1 is insoluble under our AP conditions.

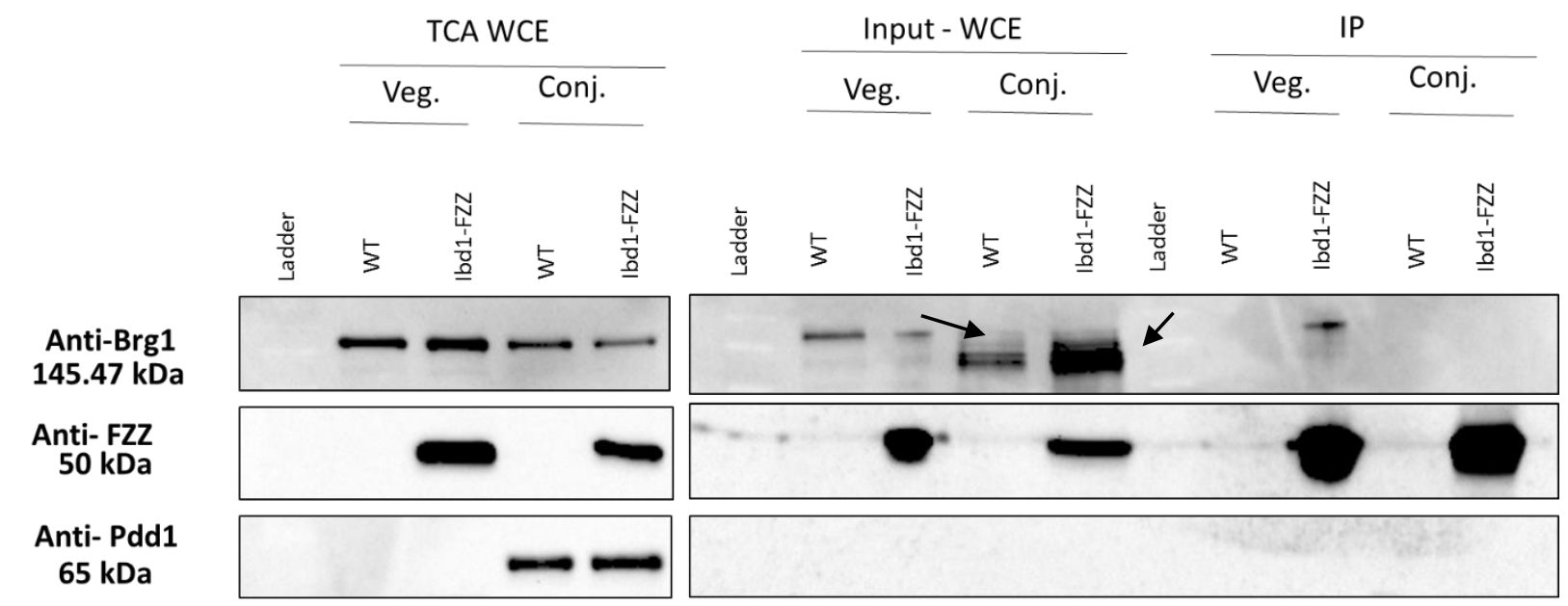

Figure 24: SDS-PAGE to equilibrate the relative amount of Ibd1 and Pdd1p solubility. In IP samples the relative amount of interacting Brg1 to Ibd1 (Anti-FZZ) is less. The arrows point to where degradation of Brg1 was observed. Anti-Pdd1 recognizes the TCA WCE and not the input WCE confirming that Pdd1 is insoluble. 


\subsection{Ibd1 recognizes multiple rather than single PTM}

An important question raised by the previous analyses is whether Ibd1's bromodomain is functional. My next objective was to determine if the bromodomain of Ibd1 was functional. In order to do so, I engineered a synthetic Ibd1 gene to express a recombinant 6XHis-Ibd1 in E.coli BL21 (D3). The purified recombinant protein was used in a commercially available histone peptide array to assess Ibd1's binding interactions with various PTM. The array contained 384 sites with single and combinations of PTM. The principle behind use of this array is described in the materials and methods section.

\subsubsection{Strain generation}

In order to begin to understand the function of Ibd1 and to determine if its bromodomain recognizes a specific histone post-translational modification I first engineered a synthetic gene to express a 6XHis-Ibd1 recombinant protein in E.coli BL21 (D3).

\subsubsection{DNA optimization}

The first steps was DNA optimization, since Tetrahymena uses a non-universal genetic code where UAA and UAG specify glutamine and not stop codons as in E.coli. Therefore in order to express a full length Ibd1 protein in E.coli, I engineered a synthetic gene to use appropriate codons for glutamic acid. In addition, I took the opportunity to optimize codon usage for expression in E.coli BL21. The engineering of the synthetic gene was performed by an external provider (BioBasics Inc.) and provided in a pUC57 plasmid.

\subsubsection{Amplification of synthetic Ibd1}

After DNA optimization of Ibd1, PCR was carried out using the pUC57 plasmid containing the Ibd1 synthetic gene as template and the used primers are described in Appendix 3. To assess success of the PCR, the samples were run in an agarose gel electrophoresis following the methods mentioned above (Figure 25). 


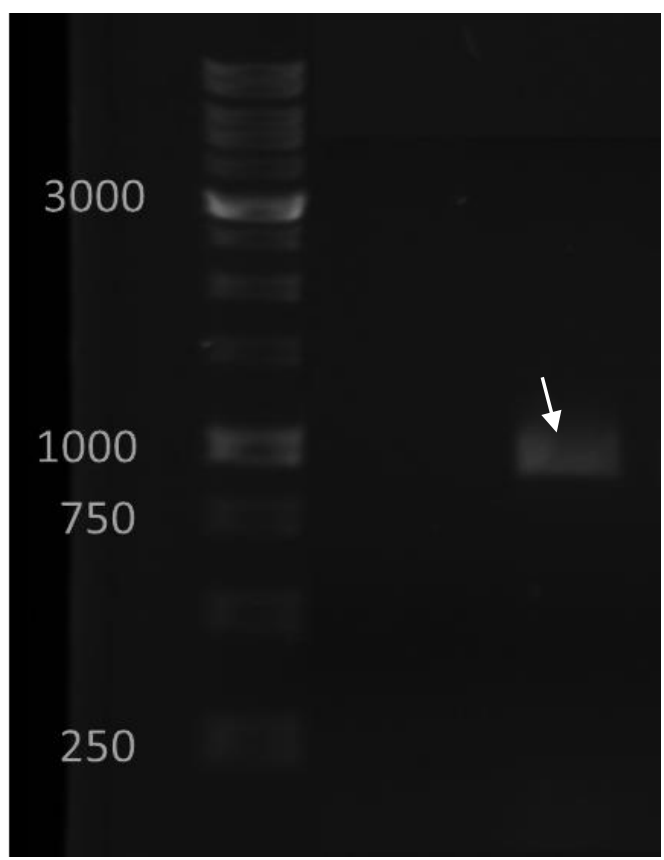

Figure 25: PCR of Synthetic Ibd1.

Arrow shows that the PCR product migrated the expected distance, corresponding to $813 \mathrm{bp}$.

4.17.1.3 Restriction digestion, ligation into expression vector and transformation into E.coli DH5 $\alpha$ followed by transformation into E.coli BL21 (DE3)

The PCR products and a pET28 plasmid (expression plasmid) were double digested with NdeI and BamHI followed by cleaning and gel purification respectively and ligation. The ligated DNA was transformed into E.coli DH5 $\alpha$ strains and the success of the cloning was confirmed by Sanger sequencing. The pET28 plasmid containing the Ibd1 synthetic gene was then used for transformation into E.coli BL21 (DE3).

\subsubsection{Inducing expression of Ibd1 in E. coli BL21 (DE3)}

The transformed E.coli BL21 (DE3) containing were grown in media containing kanamycin +/- IPTG over a period of 4 hours. Whole cell extracts by sonication, SDS loading dye following by incubation in boiling water were made. I took samples at 1 hour time intervals starting from time 0 to 4 hours post induction. I ran the samples against a ladder in an SDSPAGE. I applied Fast SeeBand Protein stain solution to the gel following electrophoresis (Figure 26). One the stained gel (Figure 25) a band can be seen at the expected size for 6xHIS-Ibd1 that 
is not present at Time 0 but is present at the other times indicating that I successfully expressed the protein.

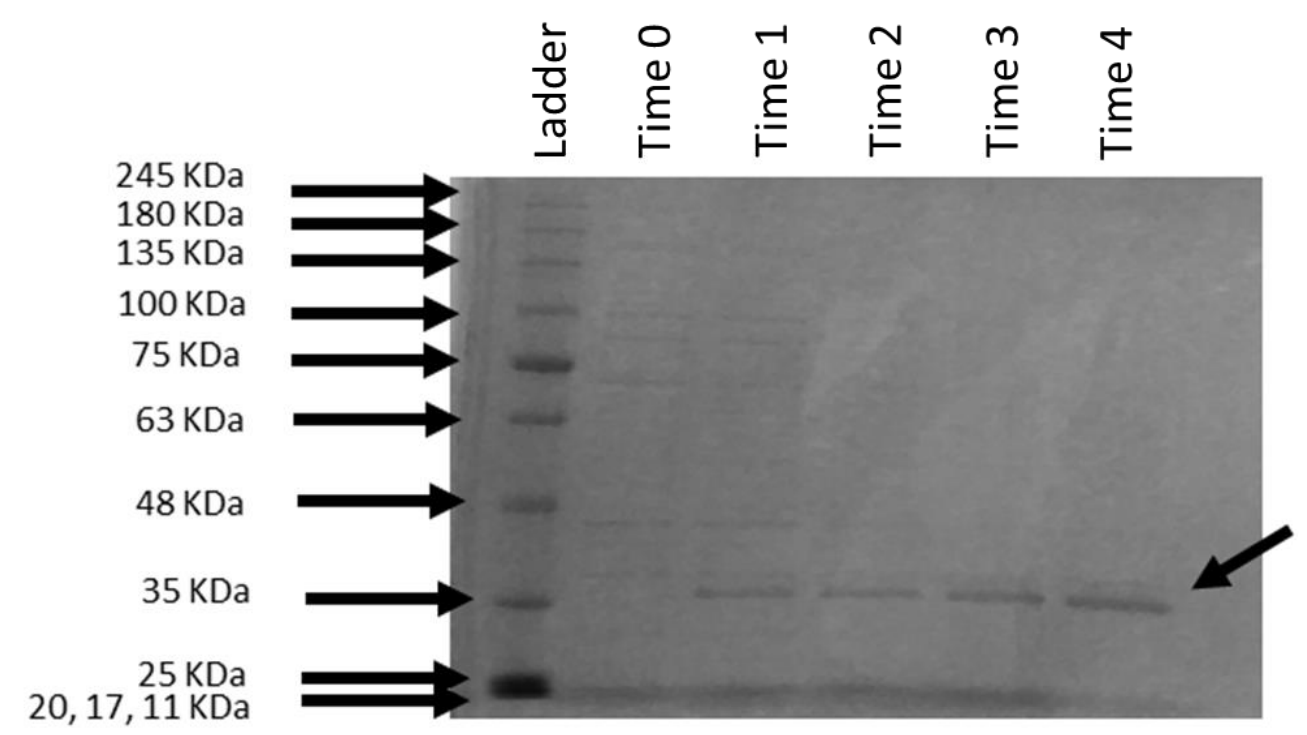

Figure 26: Inducing expression of Ibd1 in E.coli BL21 (DE3).

The arrow shows the Ibd1 predicted molecular weight is $32 \mathrm{kDa}(32 \mathrm{kDa}+1 \mathrm{kDa}$ for 6 XHis-Tag)

\subsubsection{Affinity Purification of 6XHis-Ibd1 from BL21}

Extraction of the 6XHis-Ibd1 was done using Ni-NTA Fast Start Kit (Qiagen). The recombinant protein was purified under native conditions. The input for the kit was $3 \mathrm{~mL}$ of cell pellet from IPTG induced E.coli BL21 (DE3). The extraction kit has many flow through, wash and elution steps which are described in the materials and methods section. I collected $5 \mu \mathrm{L}$ of each flow through, wash and elution fraction in separate tubes. The $5 \mu \mathrm{L}$ aliquots from each fraction were mixed with $5 \mu \mathrm{L}$ of SDS loading dye and stored at $-20^{\circ} \mathrm{C}$ for subsequent analysis with SDS-PAGE. The aliquots from each of the fractions were run in an SDS-PAGE. The gel was then stained with Fast SeeBand Protein stain solution to confirm the presence of 6XHis-Ibd1 in the samples (Figure 27). 


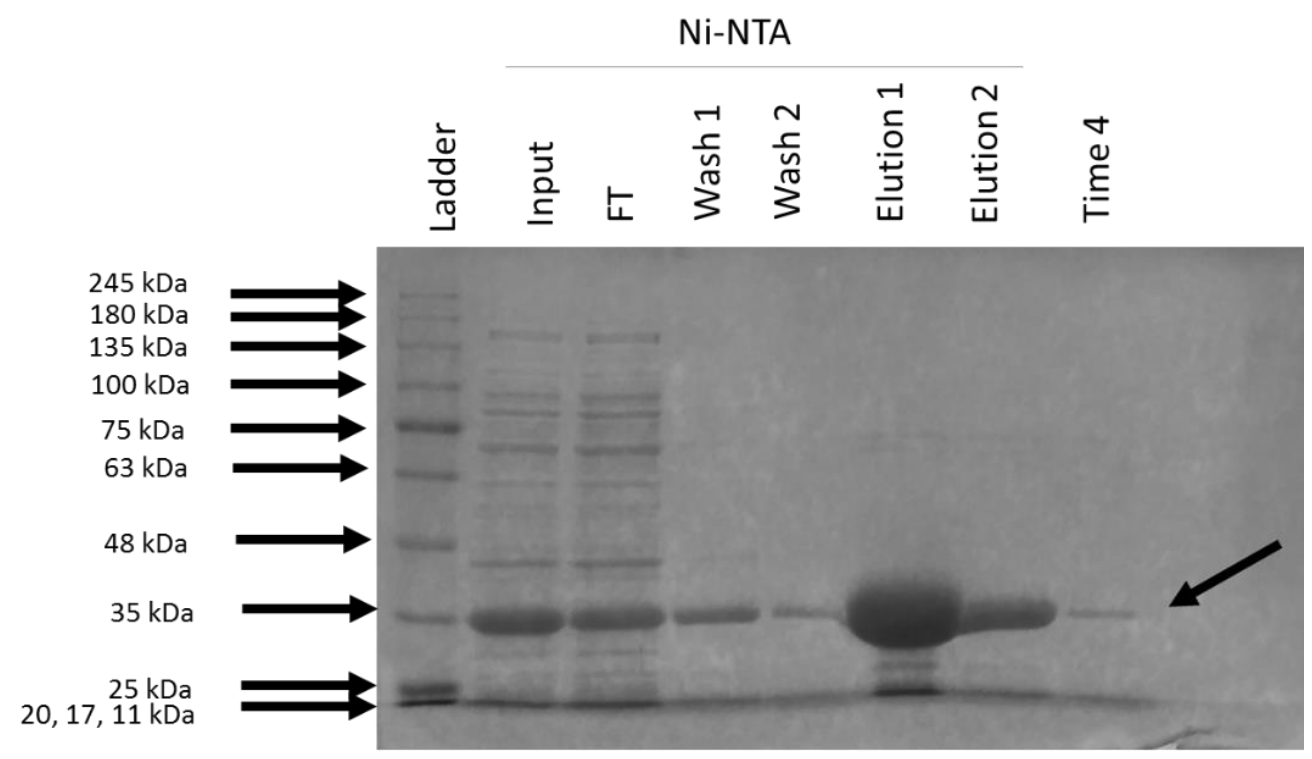

Figure 27: Purification of Expressed 6XHis-Ibd1

The arrow shows the Ibd1 predicted molecular weight is $32 \mathrm{kDa}(32 \mathrm{kDa}+1 \mathrm{kDa}$ for $6 \mathrm{XHis}-\mathrm{Tag})$

4.17.3.1 Buffer exchange by size exclusion chromatography

To obtain the 6XHis-Ibd1 in a buffer free from imidazole appropriate for downstream applications, I used buffer exchange which is based on size exclusion chromatography. I used the elutions 1 and 2 from the Ni-NTA purification and the new elution was obtained in 8 different fractions. Each of the 8 samples were run in an SDS-PAGE, followed by staining of the gel by Fast SeeBand Protein stain solution.

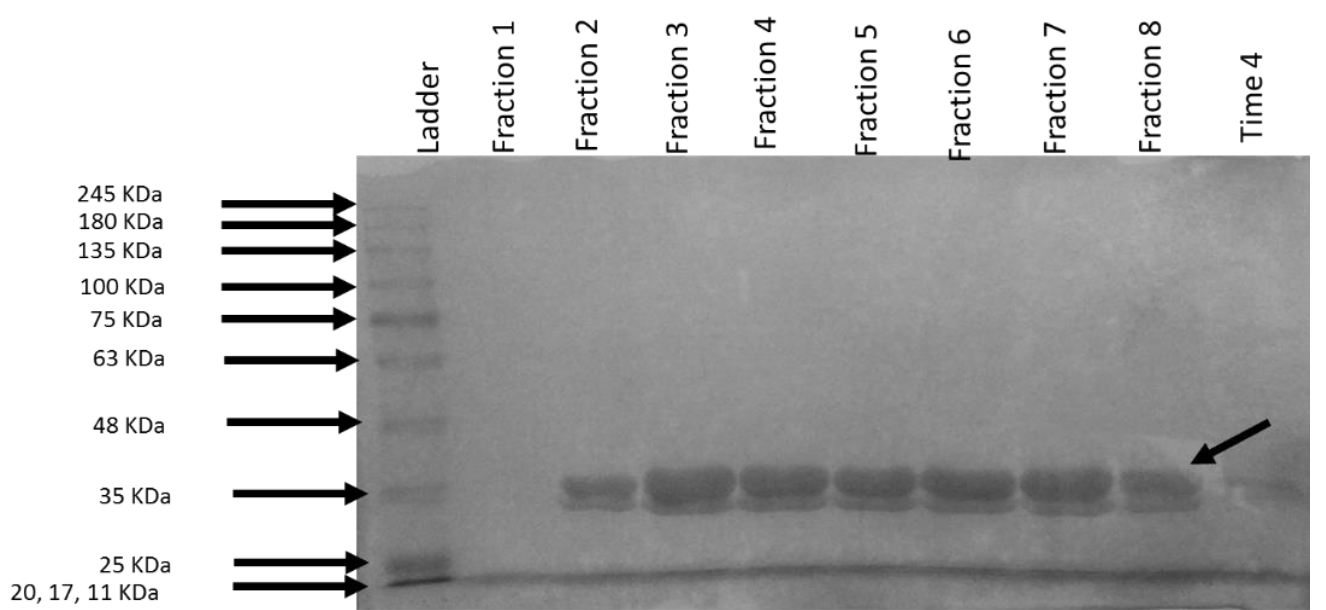

Figure 28: Buffer exchange by size exclusion chromatography

The arrow shows the Ibd1 predicted molecular weight is $\sim 32 \mathrm{kDa}(32 \mathrm{kDa}+1 \mathrm{kDa}$ for 6 XHis-Tag) 


\subsubsection{Functional characterization of Ibd1}

I used 200nM final concentration of the 6XHis-Ibd1 recombinant protein and incubated it with the array in the reaction buffer provided by the kit. This was followed by blocking, anti-His incubation, HRP conjugated secondary antibody, ECL, washes and imaging of the array. I then used the Active Motif software provided with the histone peptide array to obtain a detailed analysis of the array sites that delivered the highest signal intensities. A high intensity indicates that Ibd1 has a high affinity for this (these) PTM. The sites in the array contain 384 multiple peptides representing $\mathrm{N}$-terminal domains of human histones with a single or multiple PTM. The software assigned values based on the signal intensities from the raw data (Figure 31) and depending on the specific analysis these values may vary. There are two different analyses provided by the software. First, the specificity analysis of multiple peptide average gives a

specificity factor. The specificity factor is calculated by taking the average intensity of all spots containing that PTM divided by the average intensity of all spots not containing that PTM. The ten modifications with the highest specificity factors for sites containing both single and multiple peptides are shown in Figure 32. Second, the single peptide reactivity will measure the impact of neighbouring modifications on binding specificity. The peptide reactivity analyses are shown from Figure 33 to Figure 42. For each of the peptides with highest specificity factors from the specificity analysis of multiple peptide average and the specificity analysis of single peptides, an individual analysis of single peptide reactivity was done to compare the specificity of this peptide alone compared to when it shared a site with other modified peptides. I also used the provided positive control to validate the experiment which expected patterns are in Appendix 5.

\subsubsection{Peptide array for the control (His-G9a)}

4.17.5.1 Raw data of the peptide array for the control (His-G9a)

The provided control is a recombinant human His-G9a expressed en E.coli. His represents a histidine tag. G9a is a histone methyltransferase (via its SET domain) and only recognizes mono- and dimethylated H3K9 sites (via its ankyrin repeats)(R. E. Collins et al., 
2008). As a control for this experiment, I incubated a separate array with the provided His-G9a (10nM final concentration). The His-tag antibody was used to detect the His portion of the HisG9a. G9a only recognizes mono- and dimethylated H3K9 sites. The raw data shows the signal intensities of individual interactions between the His-G9a and the sites (circles) containing mono- and dimethylated H3K9. These sites represent N-terminal domains of human histones with a single or multiple PTM. A visual comparison of signal intensities between the obtained peptide array (Figure 29) and the model control showed in Appendix 5 was made and the layout was virtually identical.

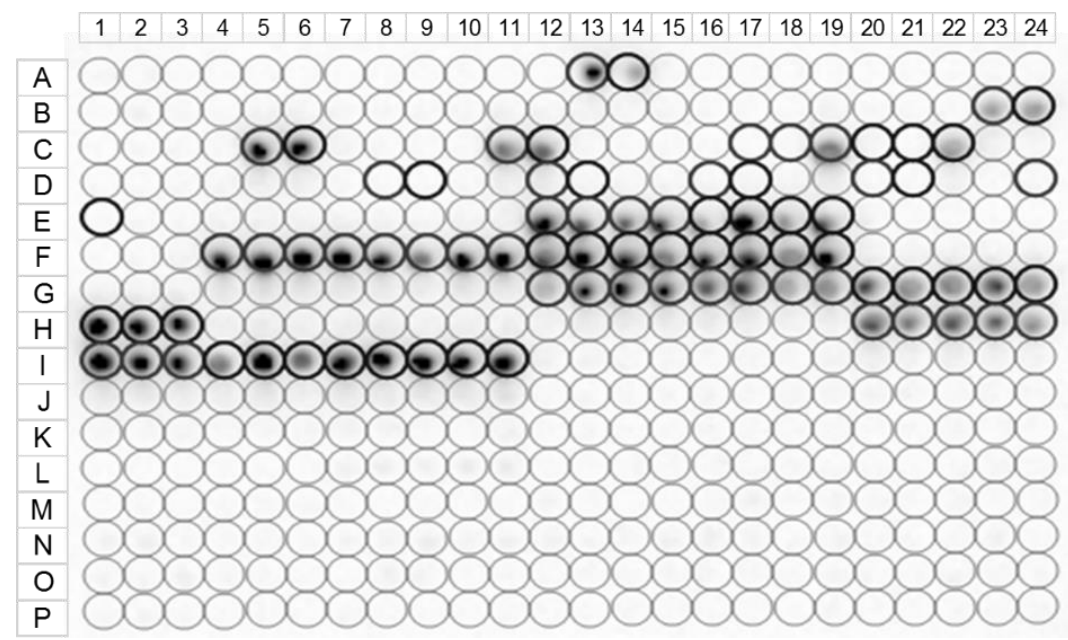

\section{Figure 29. Raw data of the peptide array for the control (His-G9a).}

The light circles represent N-terminal domains of human histones with a single or multiple PTM. The dark circles represent mono- and dimethylated H3K9 spots. The array was incubated with His-G9a and detected with the His-tag antibody. The annotations for each site are shown in Appendix 5.

\subsubsection{Specificity analysis of multiple peptide average for the control (His-G9a)}

After visual inspection of the raw data I used the software provided with the kit. The software assigned values based on the signal intensities from the raw data (Figure 28) and depending on the specific analysis these values may vary. The specificity analysis of multiple peptide average gives a specificity factor. The specificity factor is calculated by taking the average intensity of all spots containing that PTM divided by the average intensity of all spots not containing that PTM. The ten modifications with the highest specificity factors for sites 
containing both single and multiple peptides are shown in Figure 30. These values cannot be compared to the previous analyses outputted values. This analysis presents two high values: 8.2 for $\mathrm{H} 3 \mathrm{~K} 9 \mathrm{me} 2$ and 7.6 for $\mathrm{H} 3 \mathrm{~K} 9 \mathrm{me} 1$. The expected graphical analysis of the control is shown in Appendix 5. Our results match the model graphical analysis for the control thus validating Ibd1 analysis.

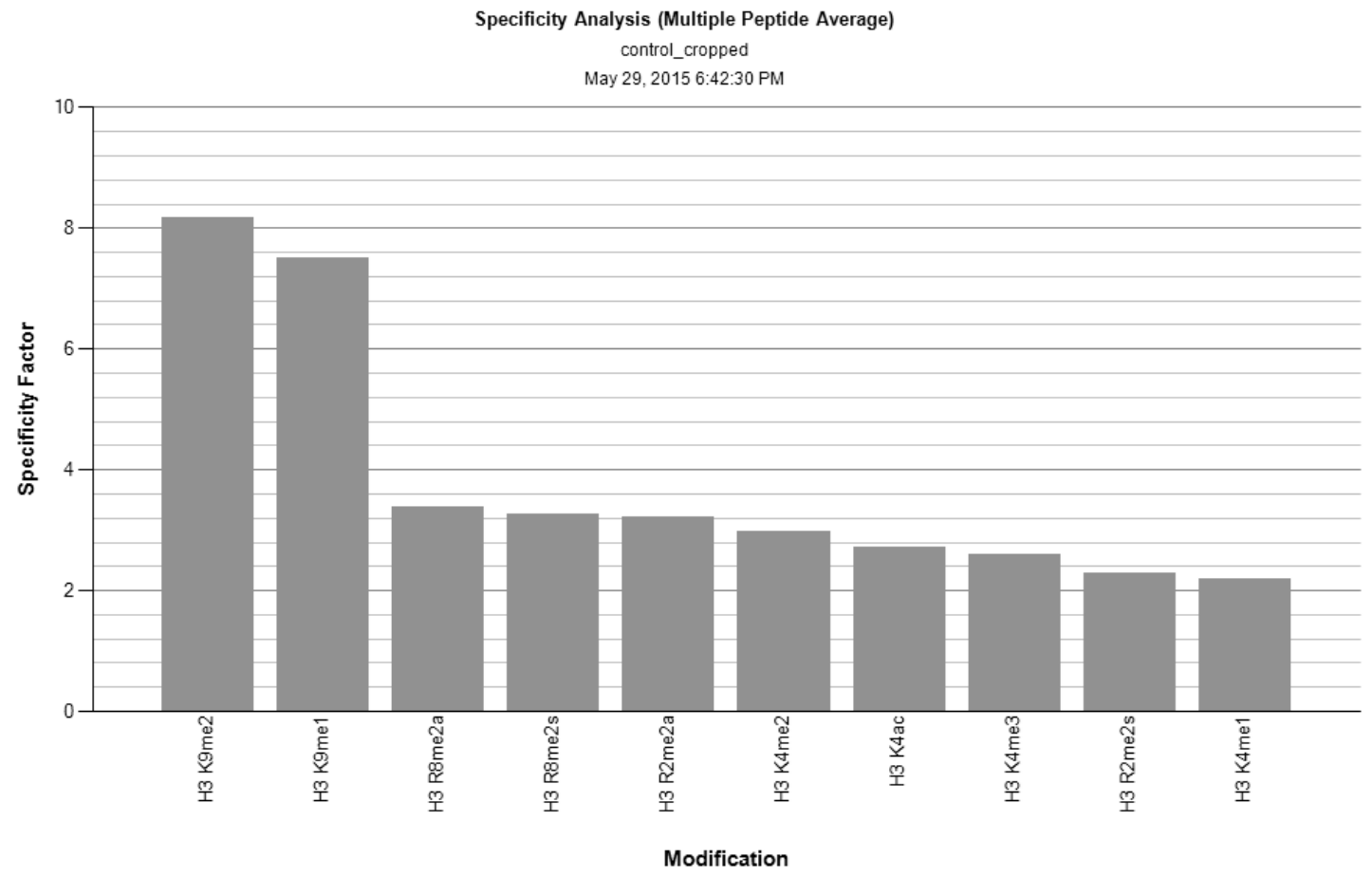

Figure 30: Multiple peptides specificity analysis for the control (His-G9a)

Peptide array used as a control for the array. The array was incubated with His-G9a and detected with the 6xHis-tag antibody.

\subsubsection{Peptide array for Ibd1}

4.17.6.1 Raw data of the peptide array for Ibd1

Now that we know the assay works, I took the recombinant 6XHis-Ibd1 and tried it in the array. The raw data shows the signal intensities of individual interactions between 6XHis-Ibd1 and the multiple peptides in each site (circles). These sites represent $\mathrm{N}$-terminal domains of human histones with a single or multiple PTM. The software assigned values based on the signal 
intensities from the raw data (Figure 31) and depending on the specific analysis these values may vary. Ibd1 did not recognize any methylated peptides like the control protein, rather it recognized almost exclusively acetylated peptides consistent with having a functional BD.

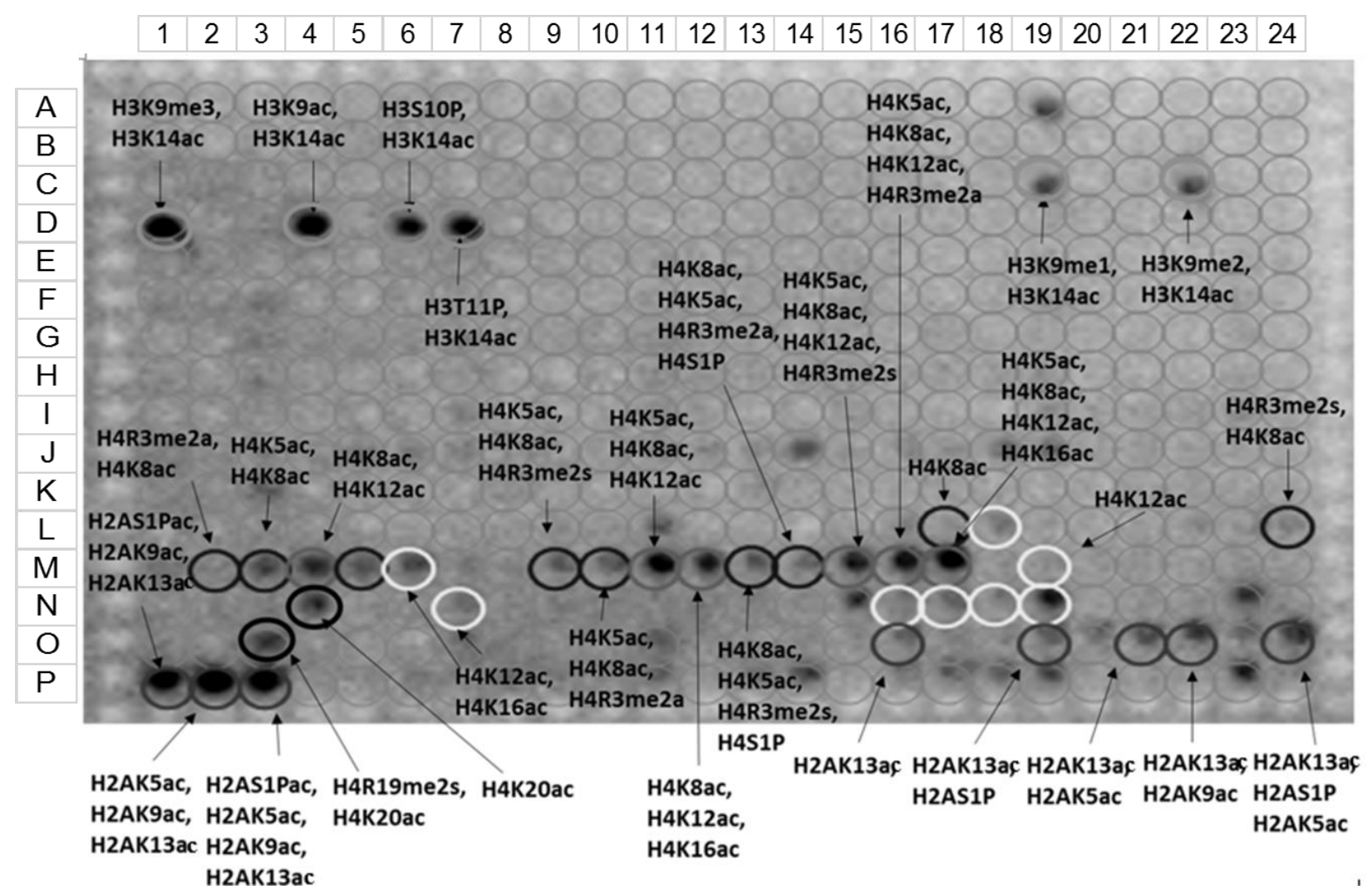

Figure 31: Ibd1 Raw data of the peptide array.

The circles represent N-terminal domains of human histones with a single or multiple PTM. The array was incubated with 6 XHis-Ibd1 and detected with the His-tag antibody. The annotations for each site are shown in Appendix 5.

4.17.6.2 Specificity analysis of multiple peptide average for Ibd1

The specificity analysis of multiple peptide average gives a specificity factor. The specificity factor is calculated by taking the average intensity of all spots containing that PTM divided by the average intensity of all spots not containing that PTM. The ten modifications with the highest specificity factors for sites containing both single and multiple peptides are shown in Figure 32. These values cannot be compared to other analyses outputted values since scales are 
different. This analysis in Figure 32 presents three high values: 8.8 for hH4K8ac, 8.4 for $\mathrm{hH} 2 \mathrm{AK} 13 \mathrm{ac}$ and 8.0 for $\mathrm{hH} 4 \mathrm{~K} 12 \mathrm{ac}$. This data suggests that the best candidate combinations to be recognized by Ibd1 include hH4K8ac and hH4K12ac. I am excluding hH2AK13ac since H2A resembles $\mathrm{H} 4 \mathrm{~N}$-terminus. I will next individually analyse each of these modifications using single peptide reactivity analysis.

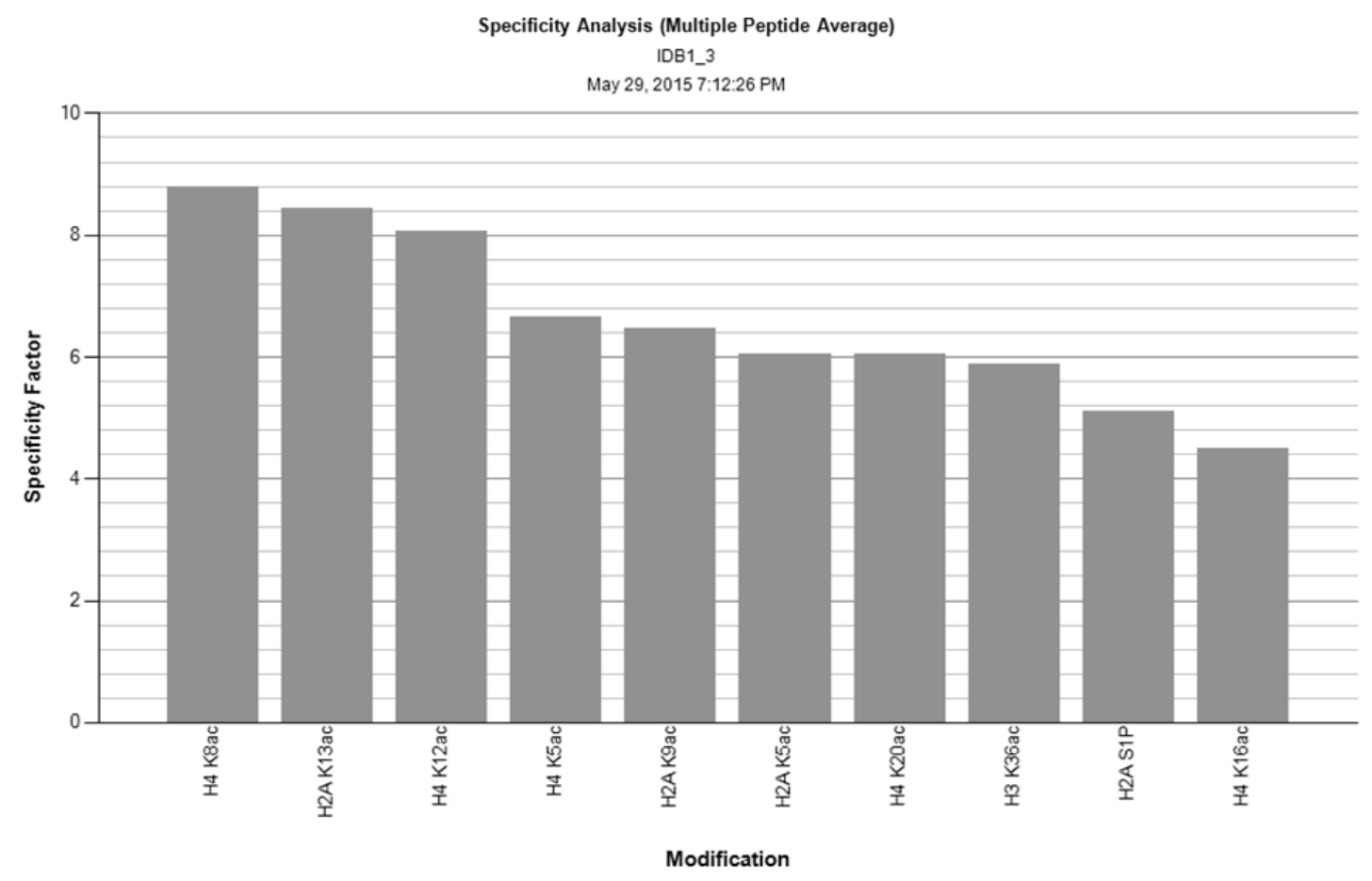

Figure 32: Multiple peptides specificity analysis for Ibd1.

Three high values: 8.8 for hH4K8ac, 8.4 for hH2AK13ac and 8.0 for hH4K12ac. This data suggests that the best candidate combinations to be recognized by Ibd1 include hH4K8ac and hH4K12ac.

4.17.6.3 Single peptide reactivity analysis for Ibd1

I will use the obtained data from the specificity analysis of multiple peptide average to individually analyse each of the outputted PTM using the single peptide reactivity analysis. The single peptide reactivity analysis will measure the impact of neighbouring modifications on binding specificity. This impact can be favorable or not for Ibd1 binding. The peptide reactivity analyses are shown from Figure 33 to Figure 42. The objective is to further understand what marks are being read by the bromodomain of Ibd1. 


\subsection{Single peptide reactivity analysis for hH4K8ac}

The individual analysis of the sites containing only hH4K8ac compared to sites containing this mark as well as additional modified peptides is seen in Figure 33. Ibd1 recognizes the human combination of H4K5ac, H4K8ac, H4K12ac (H4-Triplet) and the addition of human H4K16ac will not improve the recognition (this is depicted in Figure 33-1). (See Figure 5 for alignments). The addition of a human H4R3me2a to the H4-triplet will reduce Ibd1 recognition (Tetrahymena does not have this residue) and absence of human H4K12ac will also reduce recognition (Figure 33-2). These data suggest that the absence of any member of the H4-triplet decreases binding specificity (Figure 33-3).
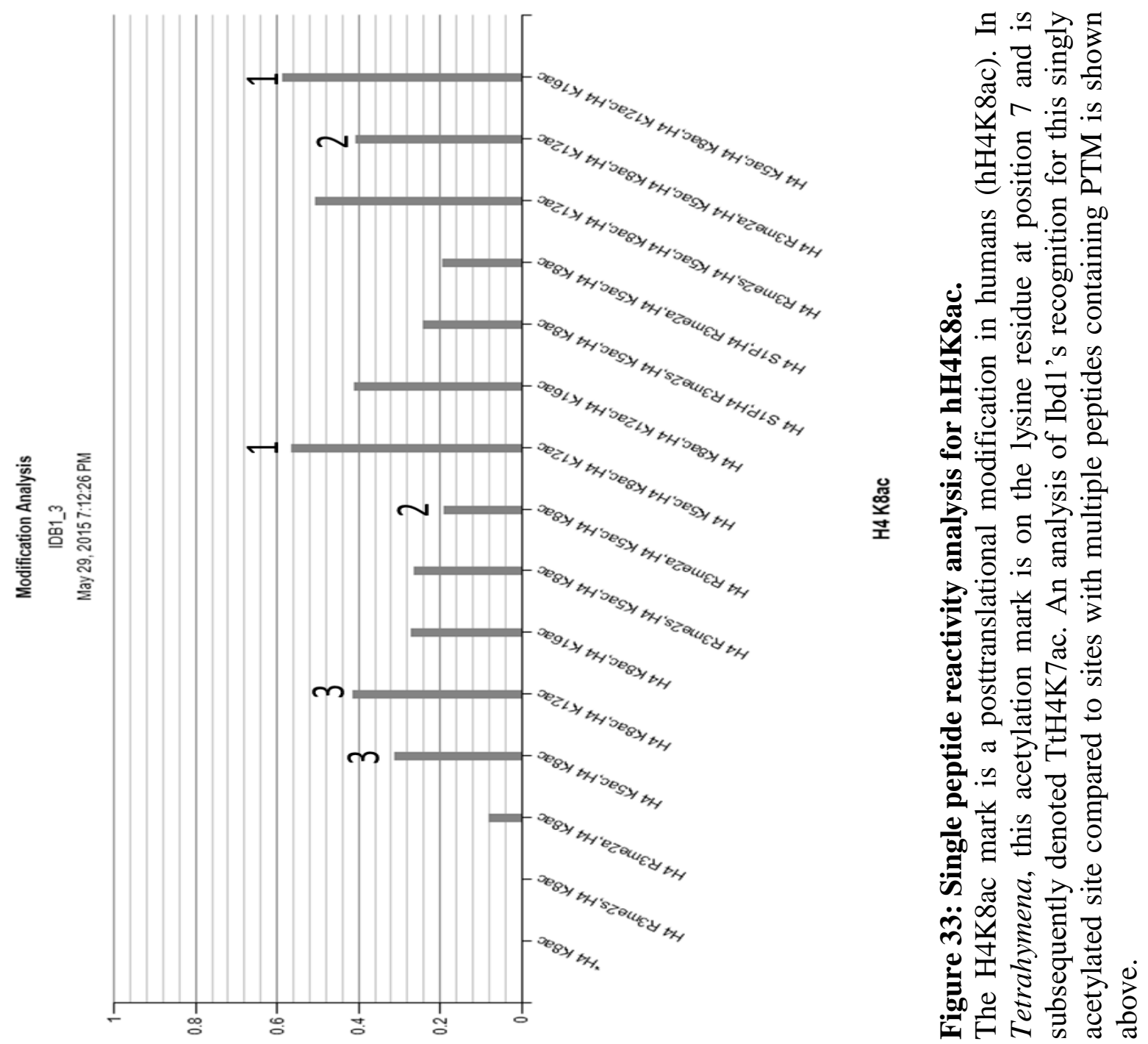


\subsection{Single peptide reactivity analysis for $h H 2 A K 13 a c$}

The individual analysis of the sites containing only hH2AK13ac compared to sites containing this mark as well as additional modified peptides is seen in Figure 34. Ibd1 also recognizes the human combination of $\mathrm{H} 2 \mathrm{AK} 5 \mathrm{ac}, \mathrm{H} 2 \mathrm{AK} 9 \mathrm{ac}, \mathrm{H} 2 \mathrm{AK} 13 \mathrm{ac}$ with the highest specificity in comparison to other sites with various PTM combinations (Figure 34-1). The addition of H2AS1P slightly decreases the binding specificity of the triplet, however its presence slightly increases the specificity when only 1 or 2 of the marks occupy one site (Figure 34-2).
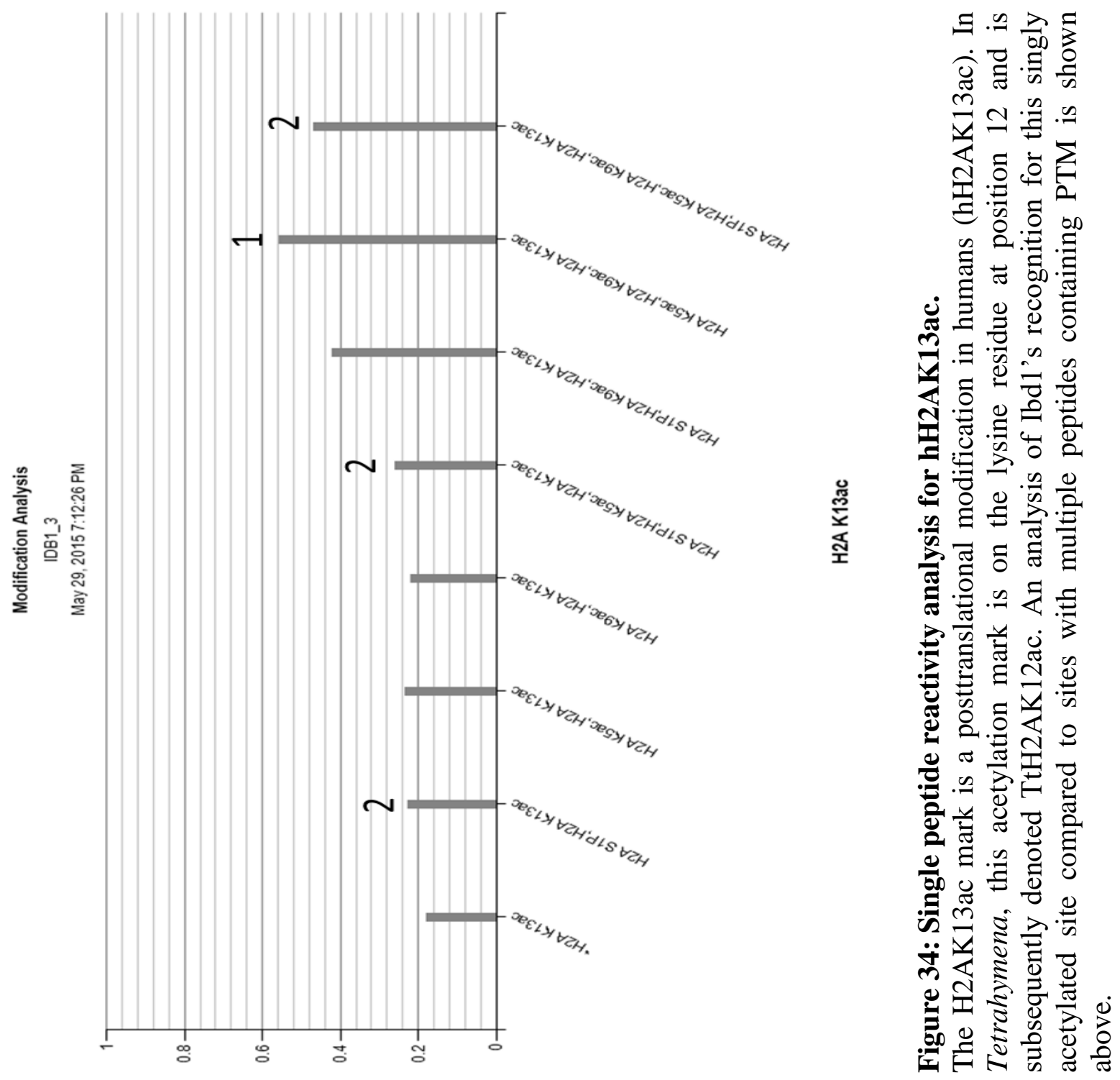


\subsection{Single peptide reactivity analysis for $h H 4 K 12 a c$}

The individual analysis of the sites containing only hH4K12ac compared to sites containing this mark as well as additional modified peptides is seen in Figure 35. Ibd1 has the highest binding specificity with triplet and quadruple acetylations (Figure 35-1). Ibd1 has very little recognition of H4K12ac on its own, however its recognition is greatly increased in the presence of another PTM, such as H4K8ac or H4K16ac (Figure 35-2). Mono-, di-, and trimethylations of H4K20 decrease Ibd1's binding specificity to H4K12ac, H4K16ac (Figure 35-3). The H4R3me2 mark seen in humans does not exist in Tetrahymena.
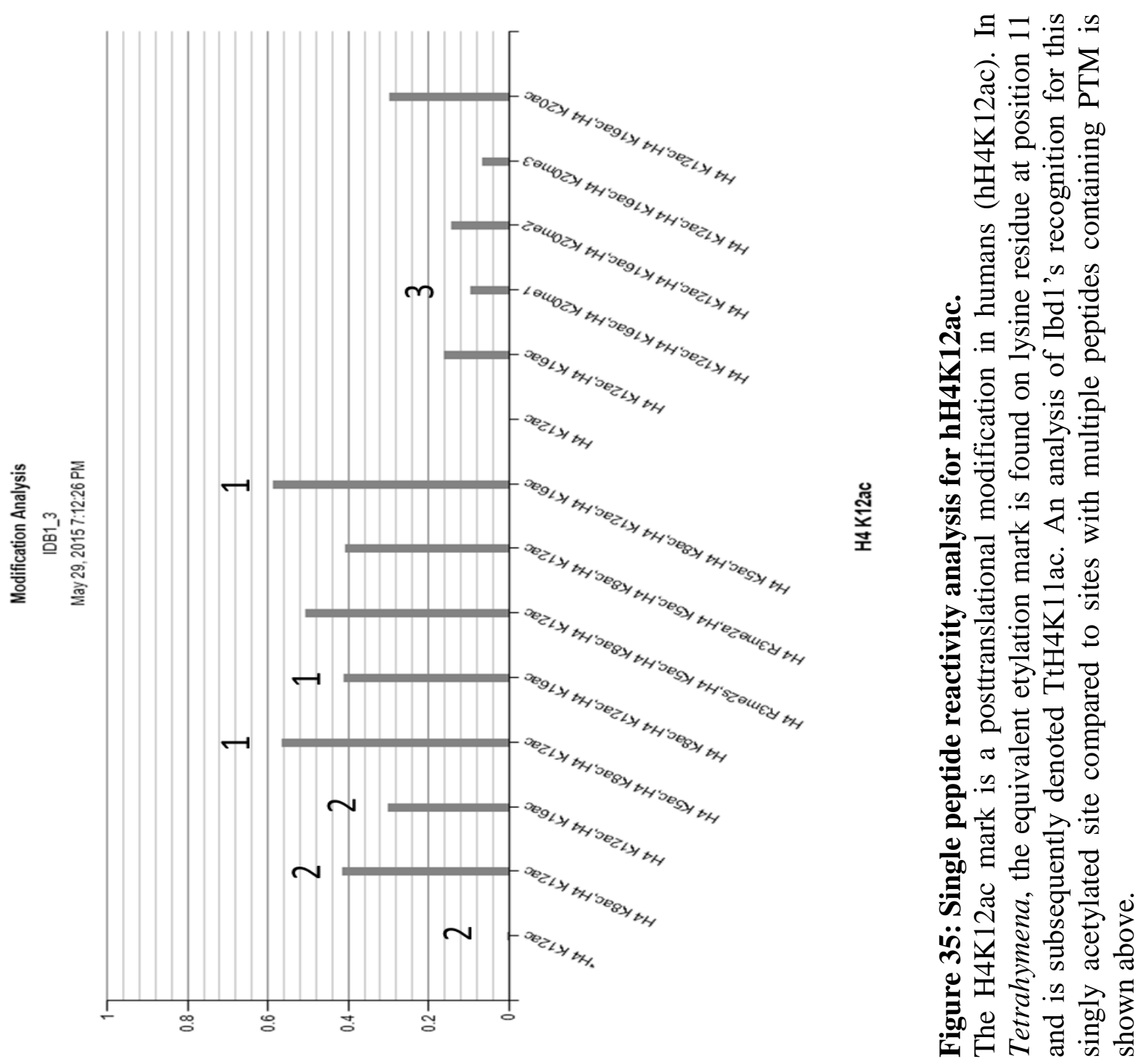


\subsection{Single peptide reactivity analysis for $h H 4 K 5 a c$}

The individual analysis of the sites containing only hH4K5ac compared to sites containing this mark as well as additional modified peptides is seen in Figure 36. Ibd1 recognizes H4K5ac with a low binding specificity (Figure 36-1). Ibd1 has a higher specificity for sites containing H4K5ac coupled to H4K8ac, and even higher for sites coupled to H4K8ac and H4K12ac (Figure 36-2). The addition of H4K16ac to the triplet only slightly increases Ibd1's binding specificity at this site (Figure 36-3).
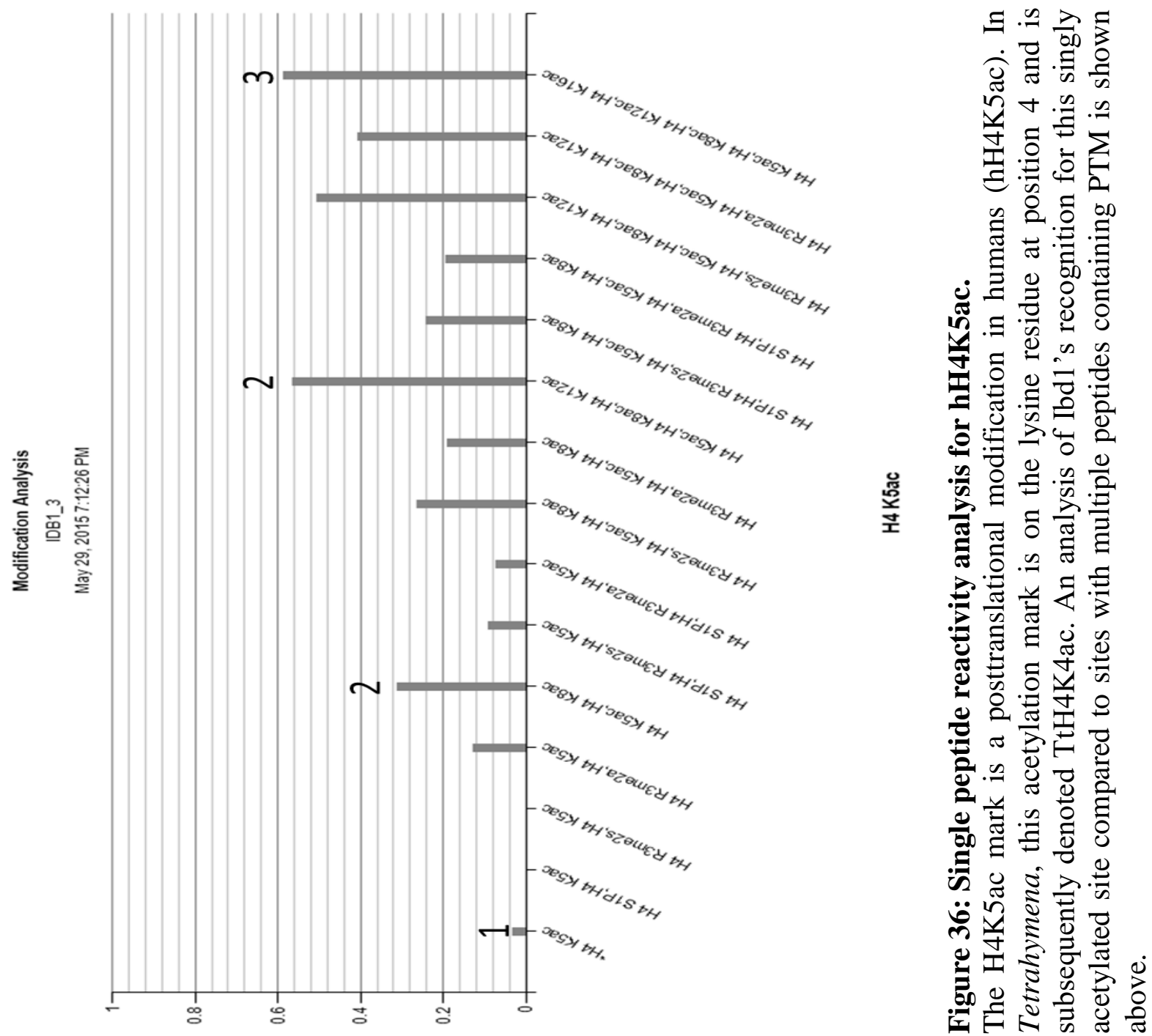


\subsection{Single peptide reactivity analysis for $h H 2 A K 9 a c$}

The individual analysis of the sites containing only hH2AK9ac compared to sites containing this mark as well as additional modified peptides is seen in Figure 37. Ibd1 recognizes the human H2AK9ac with a low binding specificity, increased slightly in combination with one other PTM (Figure 37-1). Ibd1's recognition of H2AK9ac increases greatly when in combination with 2 or 3 additional PTM; including H2AK13ac (Figure 37-2).
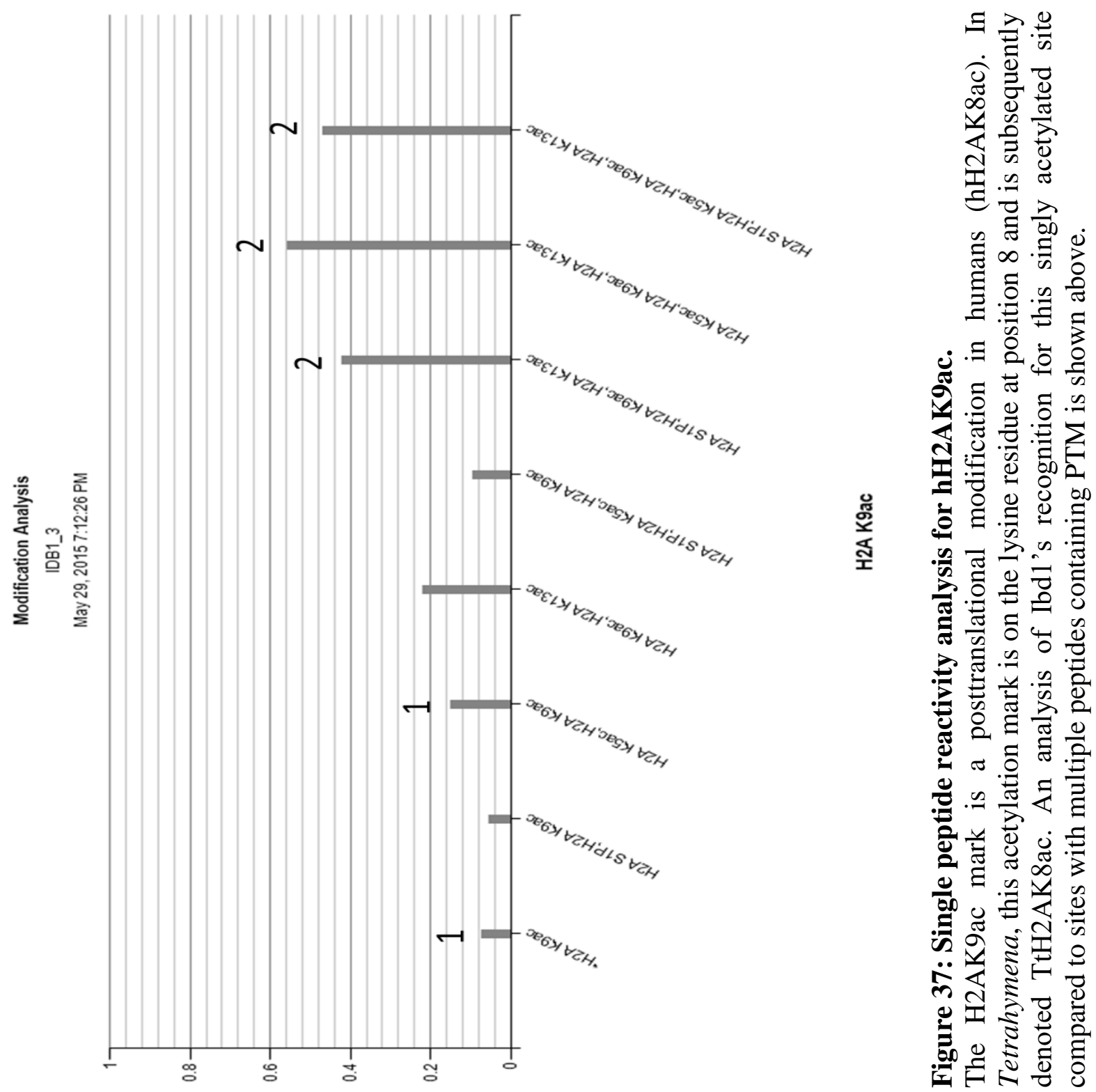


\subsection{Single peptide reactivity analysis for H2AK5ac}

The individual analysis of the sites containing only H2AK5ac compared to sites containing this mark as well as additional modified peptides is seen in Figure 38. Ibd1 has slight binding interaction with $\mathrm{H} 2 \mathrm{AK} 5 \mathrm{ac}$, and the addition of H2AS1P decreases the specificity (Figure 38-1). An additional acetylated peptide increases the binding specificity of Ibd1 for H2K5ac Figure 38-2). The combination of H2AK5ac with H2AK9ac and H2AK13ac greatly increases the binding specificity when they share a site (Figure 38-3).
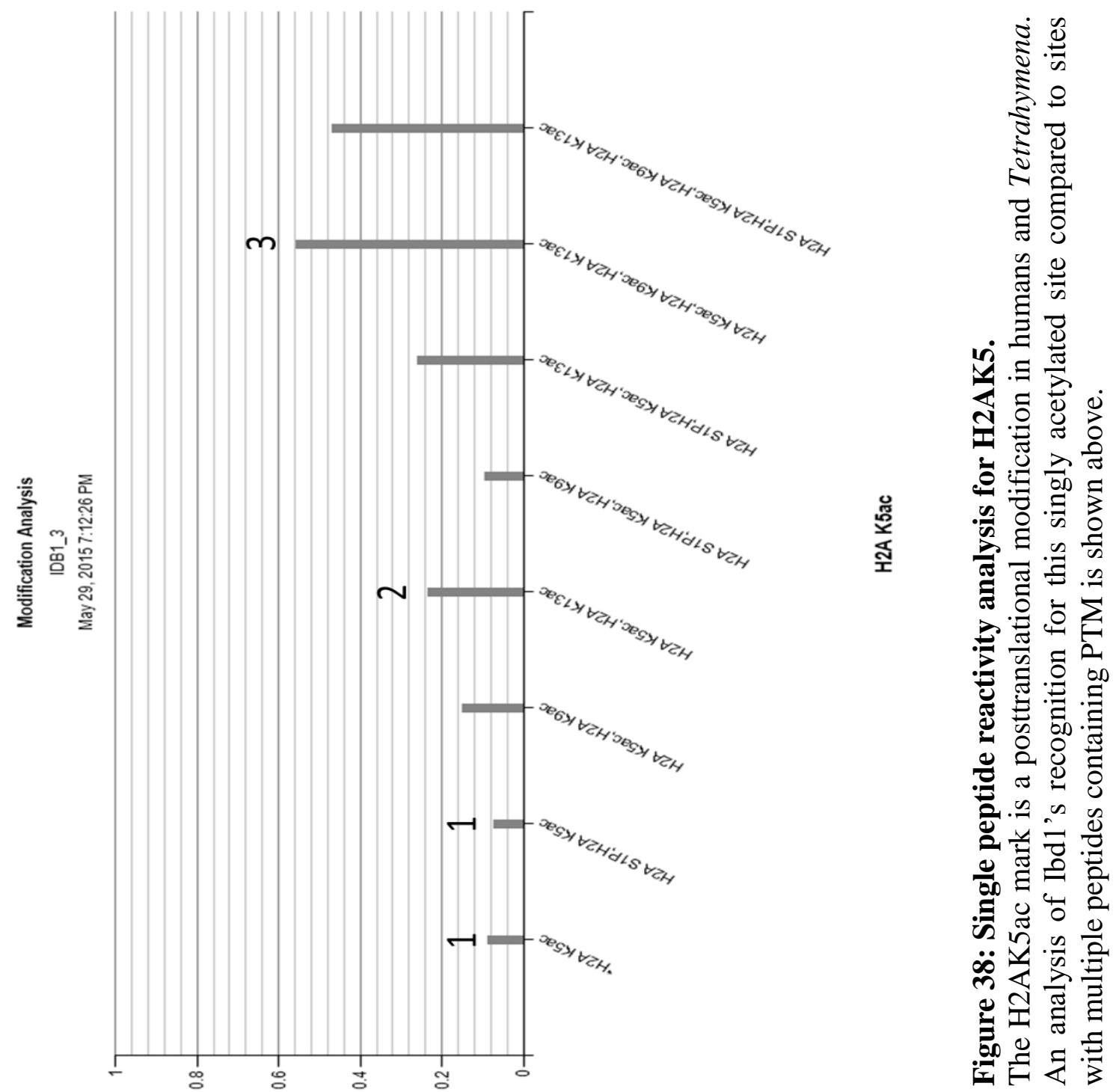


\subsection{Single peptide reactivity analysis for H4K2Oac}

The individual analysis of the sites containing only H4K20ac compared to sites containing this mark as well as additional modified peptides is seen in Figure 39. Ibd1 recognizes the H4K20ac with higher binding specificity than most of the other acetylated PTM (Figure 391). The addition of acetylated peptides to the same site does not significantly affect Ibd1's recognition of H4K20ac (Figure 39-2). Asymmetrical dimethylations decrease Ibd1's recognition for H4K20ac, however symmetrical dimethylations appear to have negligible effects on recognition (Figure 39-3).
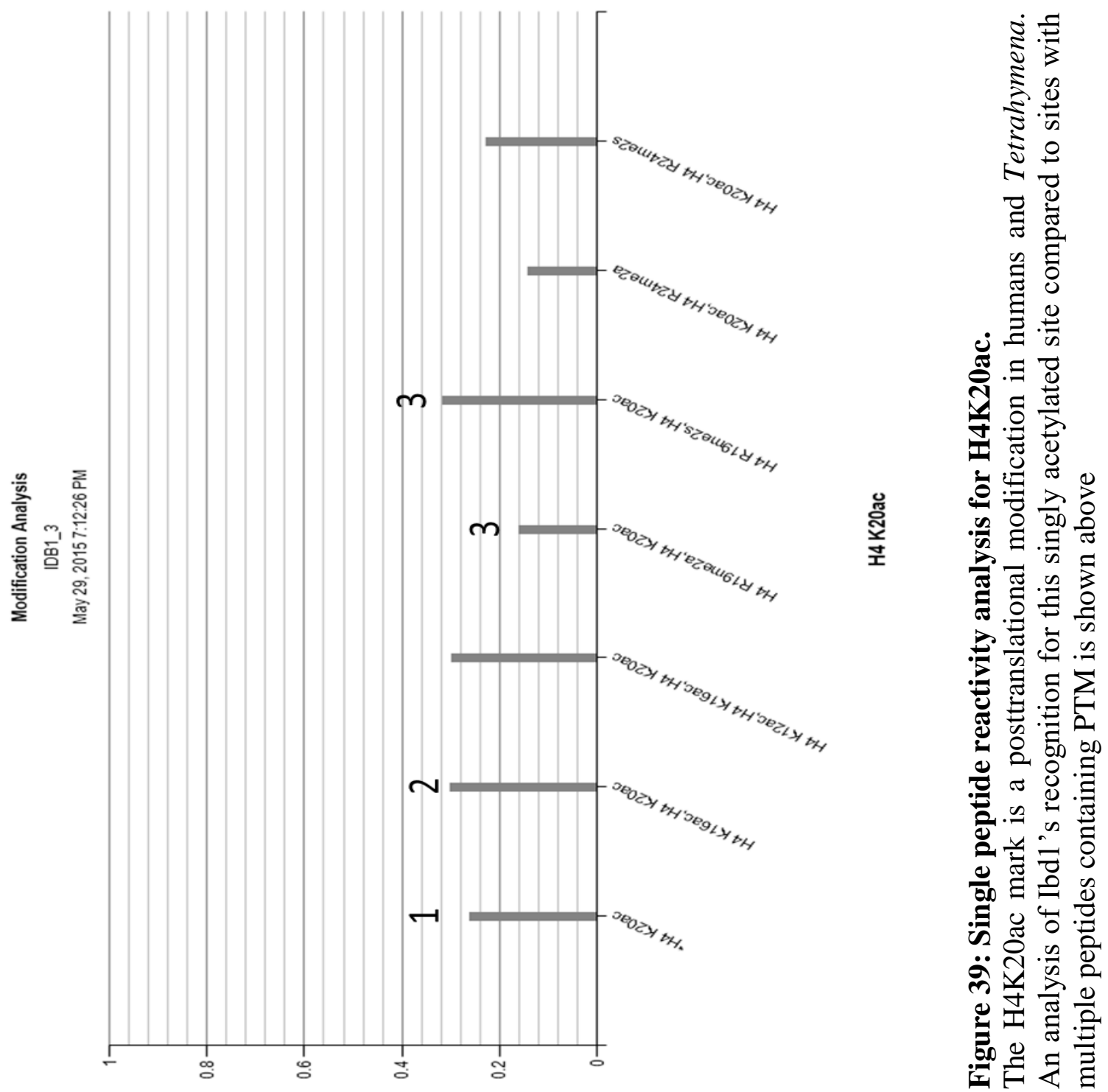


\subsection{Single peptide reactivity analysis for H3K36ac}

The individual analysis of the sites containing only $\mathrm{H} 3 \mathrm{~K} 36 \mathrm{ac}$ compared to sites containing this mark as well as additional modified peptides is seen in Figure 40. Ibd1 recognizes H3K36ac in combination with no other PTM (Figure 40-1).
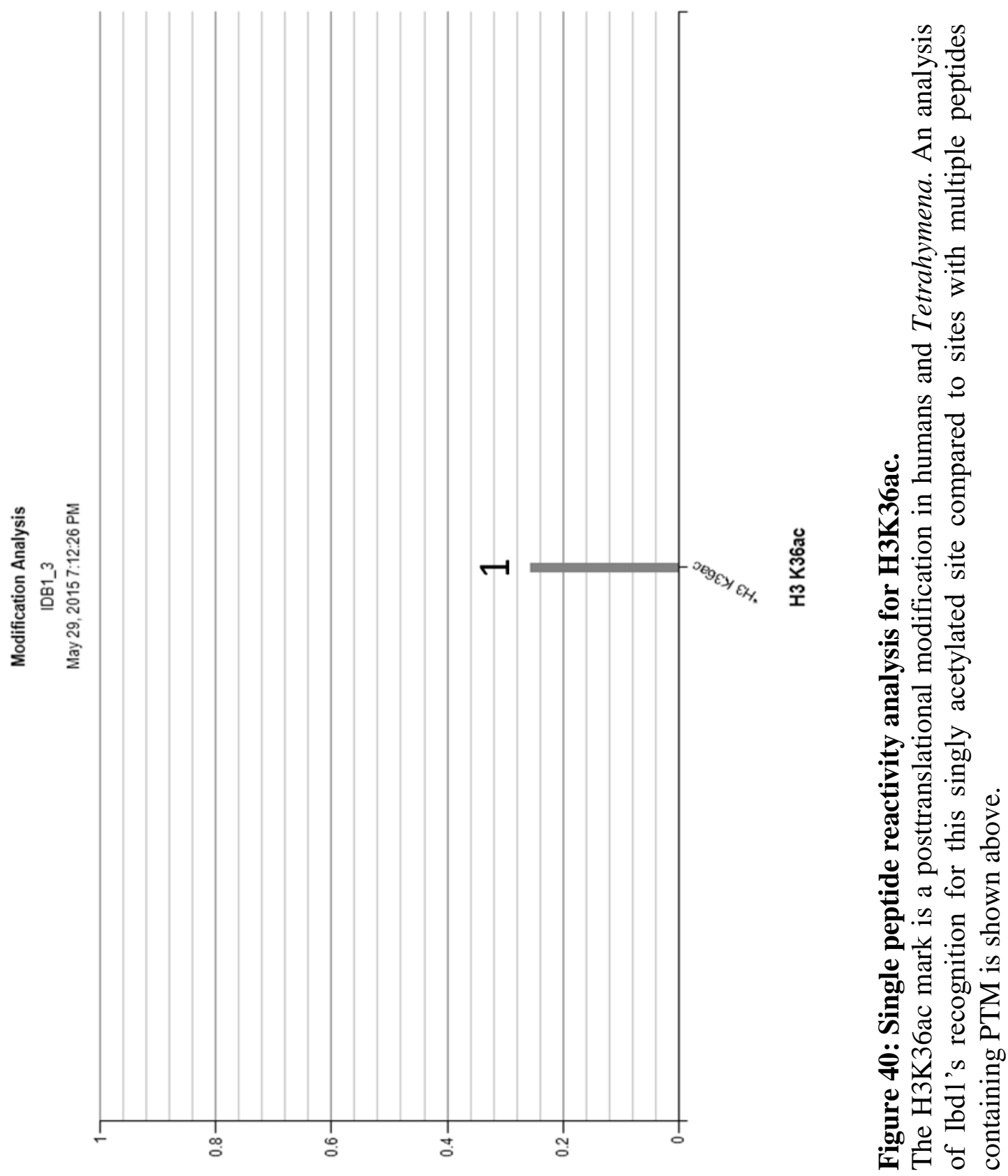


\subsection{Single peptide reactivity analysis for H2AS1P}

The individual analysis of the sites containing only H2AS1P compared to sites containing this mark as well as additional modified peptides is seen in Figure 41. Ibd1 has low recognition for H2AS1P on its own and in combination with one or both of H2AK5ac and H2AK9ac (H2AK8ac in Tetrahymena) (Figure 41-1). When in combination with H2AK13ac (H2AK12ac in Tetrahymena), the recognition increases (Figure 41-2). Ibd1 shows the greatest binding specificity for sites containing H2AS1P and the H2AK5ac, H2AK9ac, H2AK13ac triplet (H2AKac, H2AK8ac, and H2AK12ac in Tetrahymena) (Figure 41-3).
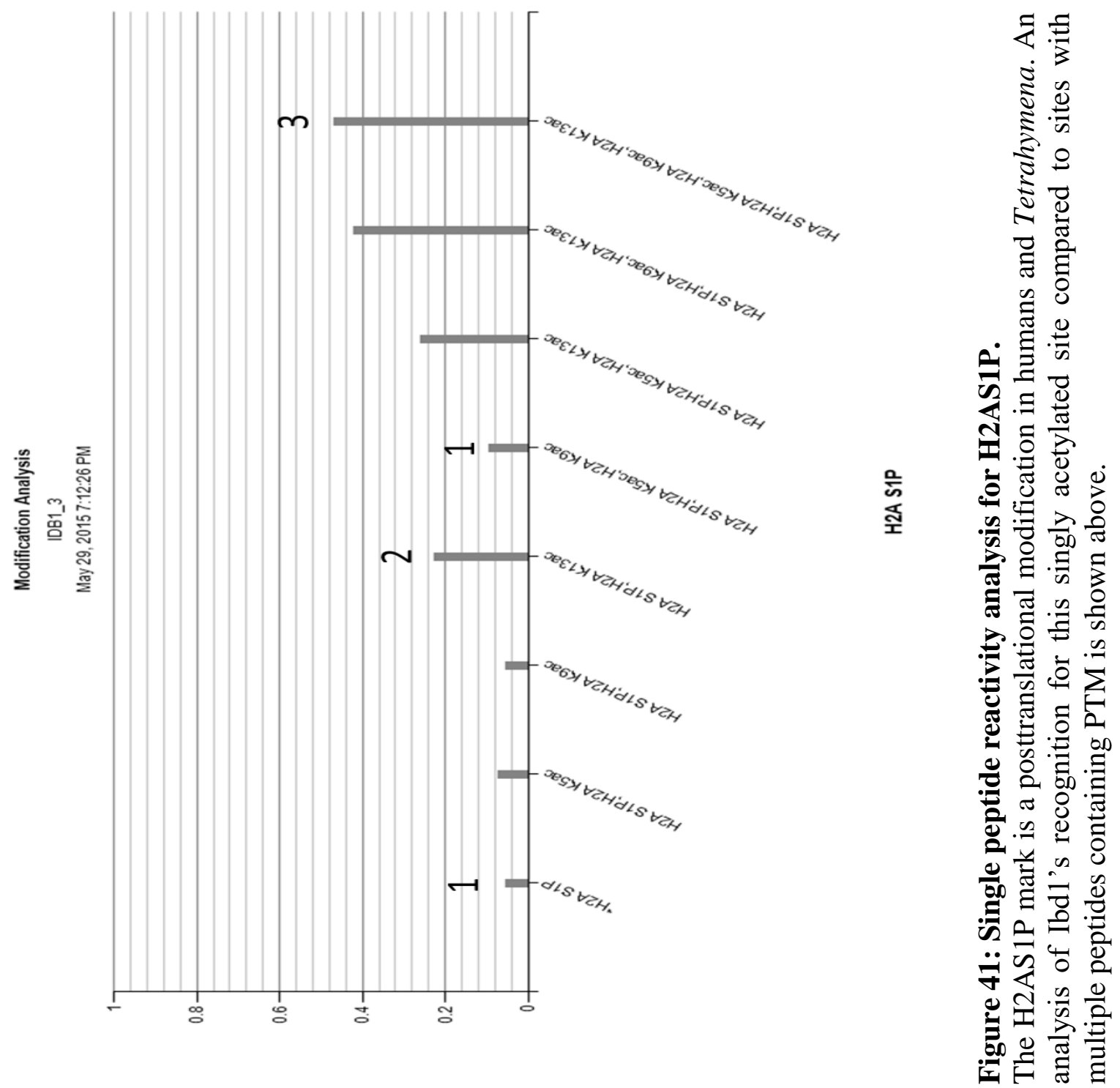


\subsection{Single peptide reactivity analysis for hH4K16ac}

The individual analysis of the sites containing only hH4K16ac compared to sites containing this mark as well as additional modified peptides is seen in Figure 42. Ibd1 does not recognize H4K16ac on its own (Figure 42-1). The addition of 1, 2 and 3 acetylated peptide(s) increasingly affects the binding specificity of Ibd1 for the site (Figure 42-2). Mono-, di- and trimethylations increase Ibd1's recognition of the site compared to H4K16ac on its own. The symmetry of the methylation does not appear to affect recognition (Figure 42-3).
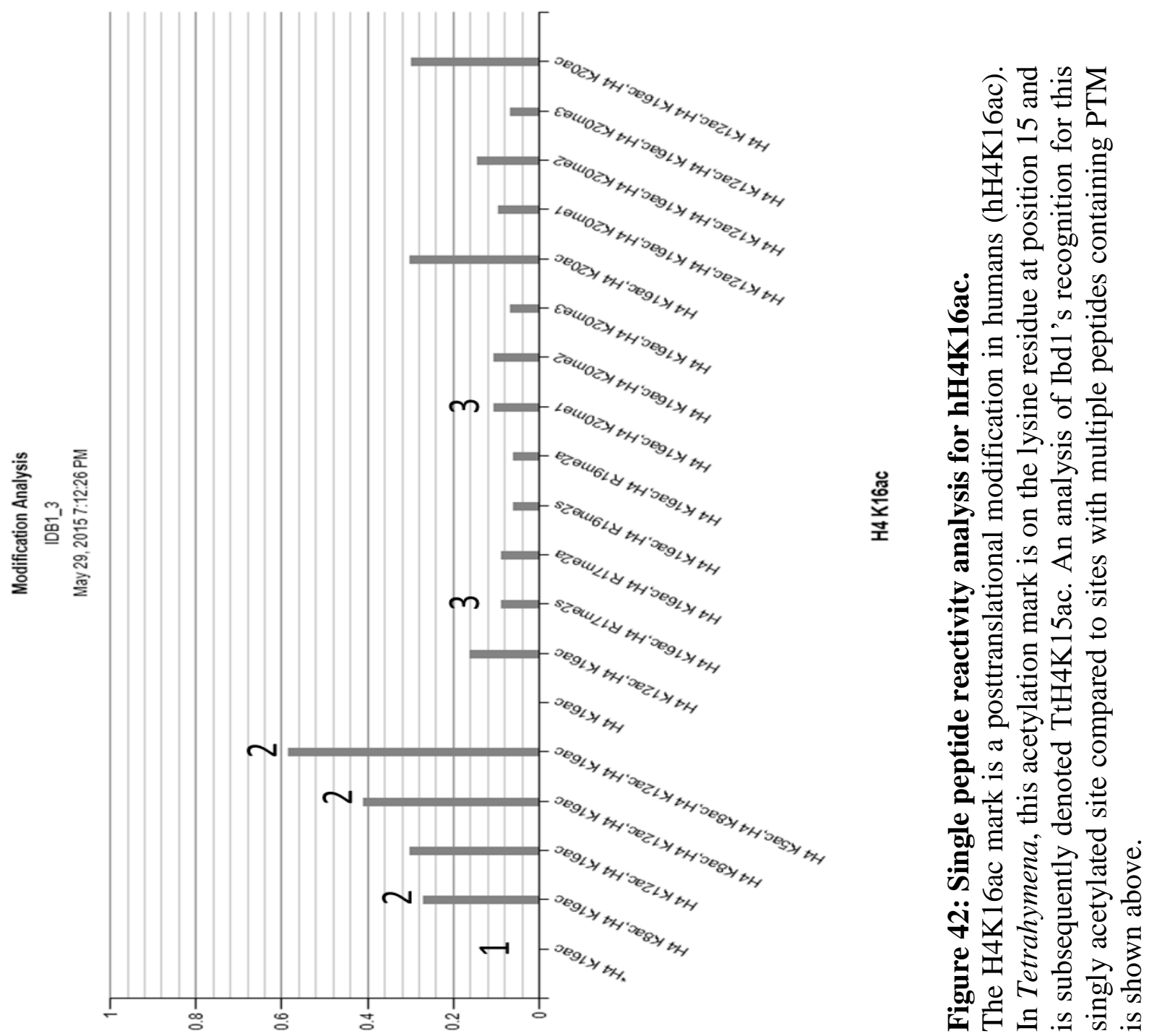
From the single peptide reactivity analysis, it can be seen that the human combination of marks that Ibd1 recognizes with the highest specificity (a value of 0.58 ) is human H4K5ac, H4K8ac, H4K12ac, H4K16ac. For these human PTM the Tetrahymena analogues will be H4K4ac, H4K7ac, H4K11ac, H4K15ac. The next highest value of 0.56 results from the hH4K5ac, hH4K8ac, hH4K12ac combination. For these human PTM the Tetrahymena analogues will be H4K4ac, H4K7ac, H4K11. The third highest value of 0.56 results from the combination of hH2AK5ac, hH2AK9ac, hH2AK13ac. For these human PTM the Tetrahymena analogues will be H2AK5ac, H2AK8ac, H2AK12ac. Based on this analysis, Ibd1's strongest binding interaction is with the PTM H4K20ac, giving a value of 0.26 , and $\mathrm{H} 3 \mathrm{~K} 36$ with a value of 0.25 . There is a clear preference of Ibd1 to multiple PTM.

\subsection{Ibd1 localizes to MAC}

During growth, I expected to find that SWI/SNF proteins would be present in the MAC but not the MIC, which were consistent with a role in transcription. During conjugation, I expected that cells would show localization again to the MAC and not the meiotic MIC similar to what was shown for TtBrg1 (Fillingham et al., 2006). If the targeted proteins show similar localization to TtBrg1, this is good evidence for common function with TtBrg1 and consistent with function in a SWI/SNF complex. Thus, TtBrg1 does not localize to MIC, which is unusual because several transcription proteins localize to meiotic MIC. Perhaps in T.thermophila the SWI/SNF complex does not function in MIC transcription but only in MAC transcription and other functions such as amitosis.

In order to further characterize the function of the TtSWI/SNF complex, I performed IF of Ibd1. Figure 43 shows that Ibd1 localizes to the MAC during growth and sexual development including meiosis, consistent with function in transcription. Fillingham et al. (Fillingham et al., 2006) showed that TtBrg1 localizes to the MAC during growth and development. This is consistent with a role in transcription during growth and development. The targeted protein (Ibd1) shows similar localization to TtBrg1, providing substantial evidence for common function with TtBrg1 and consistent with function in a SWI/SNF complex. 


\subsection{H4K8ac localizes to MAC}

The rationale of doing this experiment is based on the peptide array results that suggest that Ibd1 recognizes modifications where the PTM H4K8ac is present. If the previous statement is correct, H4K8ac should have similar localization to Ibd1. Figure 43 shows that H4K8ac and Ibd1 both only localize to the MAC during growth and sexual development including meiosis, consistent with function in transcription, consistent with a role in transcription. 


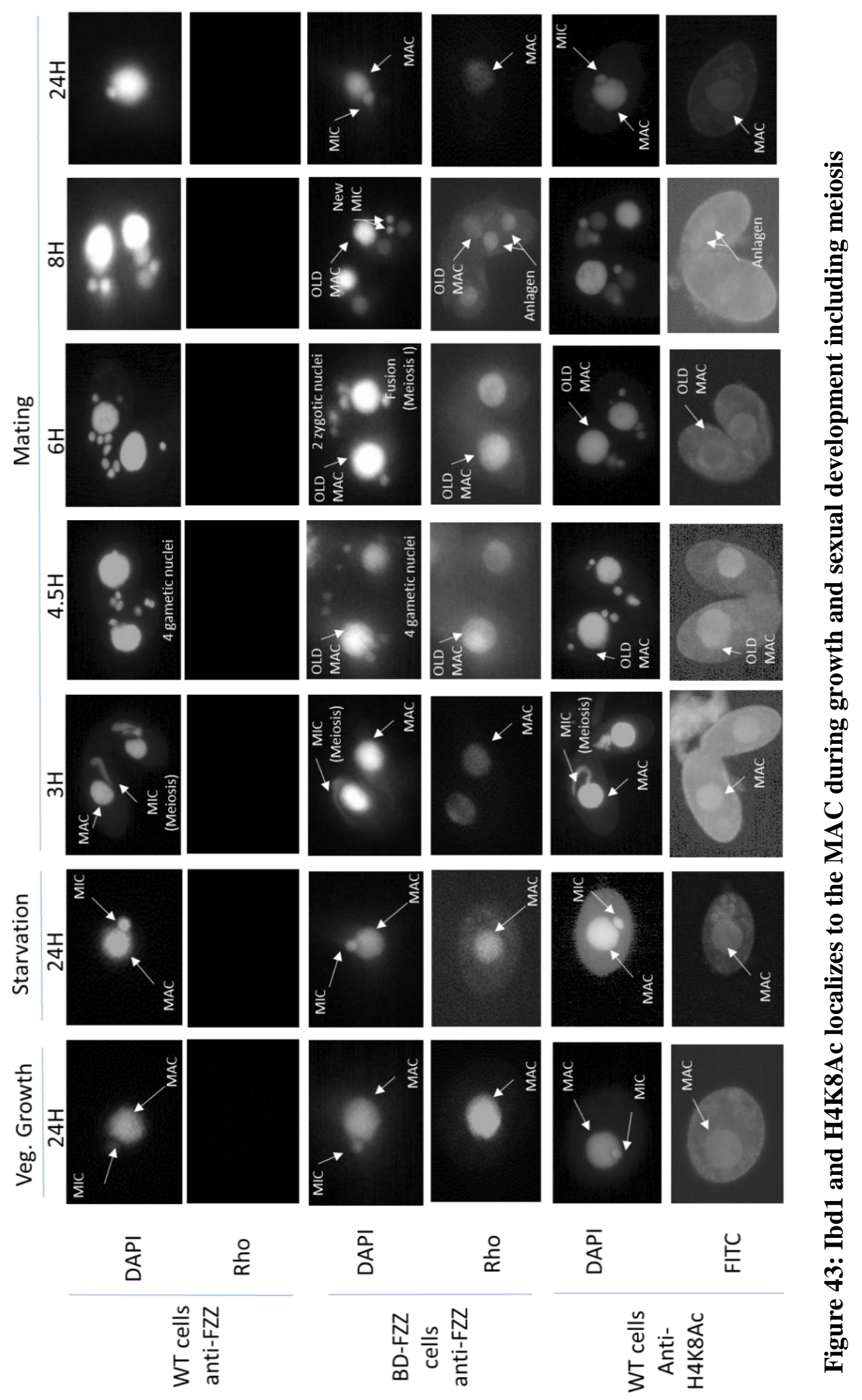




\subsection{Model of Ibd1 localization during T.thermophila's life cycle}

Figure 44 shows that Ibd1 localizes to the MAC during vegetative growth, starvation, meiosis $(3 \mathrm{H})$, gametic nuclei fusion $(4.5 \mathrm{H})$ and zygotic nuclei duplication $(6 \mathrm{H})$. At $8 \mathrm{H}$ during anlagen development, Ibd1 localizes to anlagen (new MAC) and old MAC similar to Brg1 (Fillingham et al., 2006). This is consistent with a role in transcription and new MAC development. At $24 \mathrm{H}$ when the daughter cells have formed, the expressed Ibd 1 from the parental MAC might be stable enough to localize to the MAC of the daughter cells.

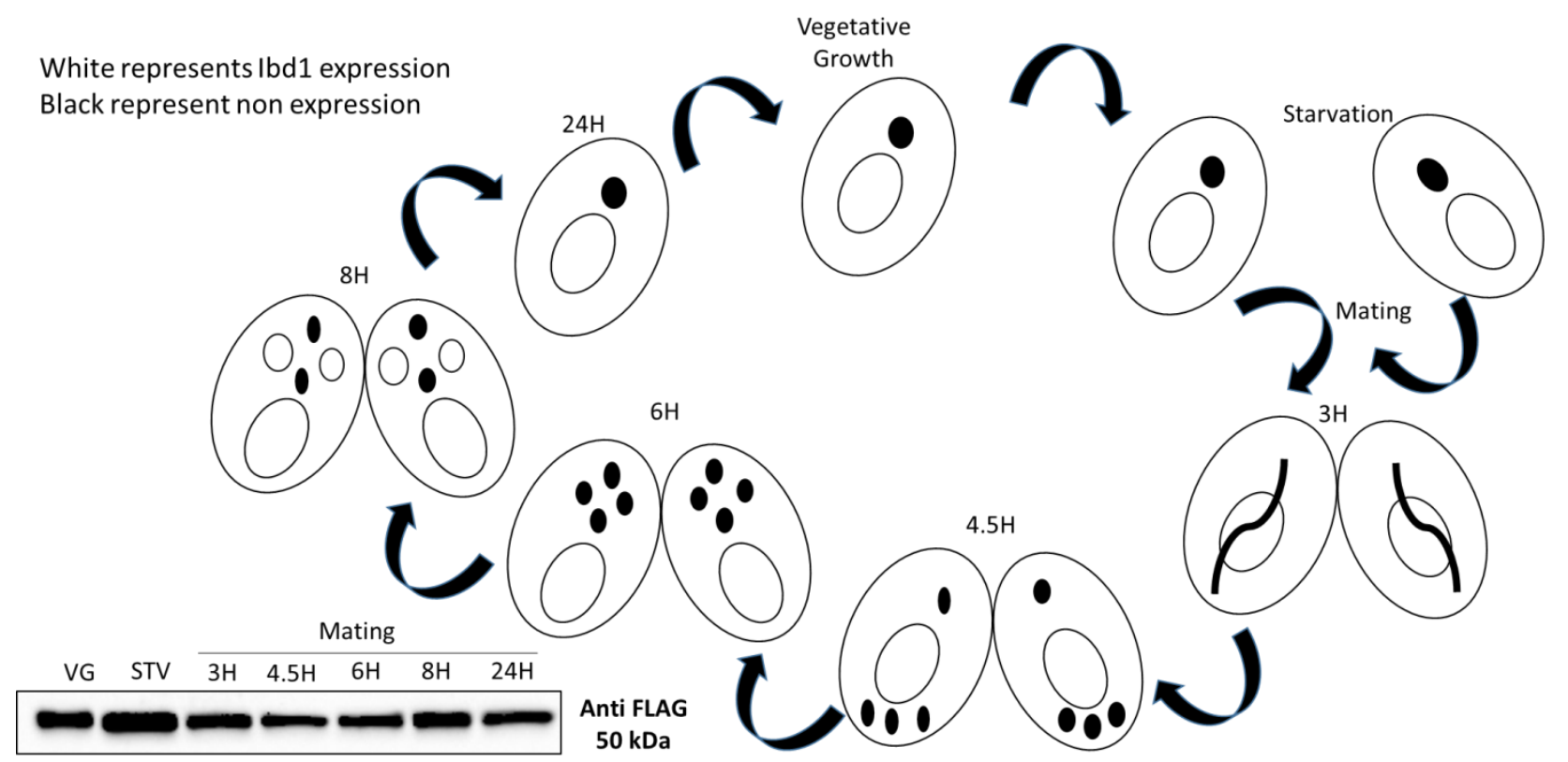

Figure 44: Model of Ibd1 localization during T.thermophila's life cycle. 


\section{CHAPTER 3 - ISWI RESULTS}

An important part of Tetrahymena's life cycle are DNA rearrangements (Madireddi et al., 1996)(Smothers et al., 1997)(Chalker et al., 1999)(Coyne, Nikiforov, Smothers, Allis, \& Yao, 1999)(Mochizuki et al., 2002)(Mochizuki et al., 2002)(Taverna et al., 2002)(Malone et al., 2005) (Y. Liu et al., 2007)(Chalker, 2008)(Schwope \& Chalker, 2014). One initial interest of our lab was to characterize chromatin related proteins involved in DNA rearrangements. I initially hypothesized that Iswil is involved in DNA rearrangements. I based this hypothesis on a publically available gene expression profile of mRNA (www.ciliate.org) for Iswi1 (Figure 45), which indicates that Iswil is highly expressed at 2 and 7 hours during conjugation. This coincides with the time during conjugation where other proteins (Figure 46) involved in DNA rearrangements are being expressed. At 2 hours, Iswil is highly expressed (Figure 45) and the meiotic transcription of long ncRNAs (scnRNA precursors) is occurring (Mochizuki et al., 2002). Therefore Iswil may be the protein that regulates the expression of long ncRNA. To answer this, I tagged the Iswi1 protein (TTHERM_00388250).

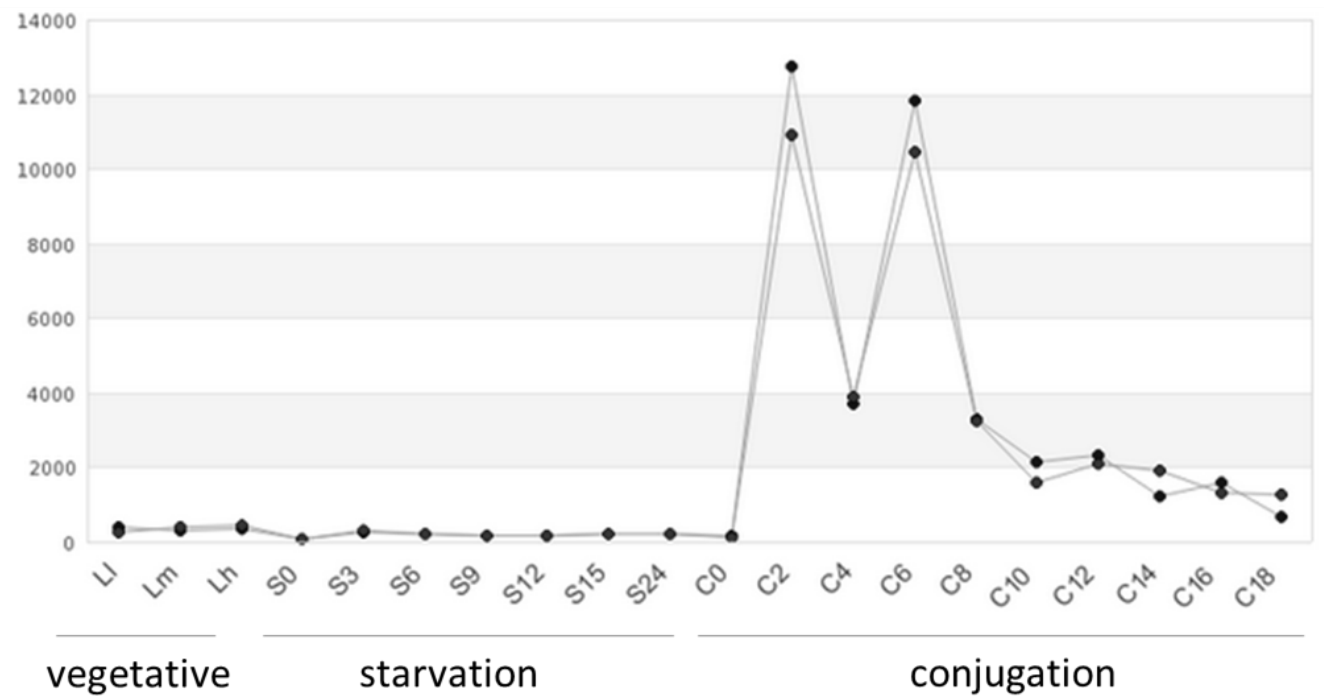

\section{Figure 45: Iswi1 gene expression profile from RNA-seq}

Tetrahymena functional genomics database from ciliate.org 


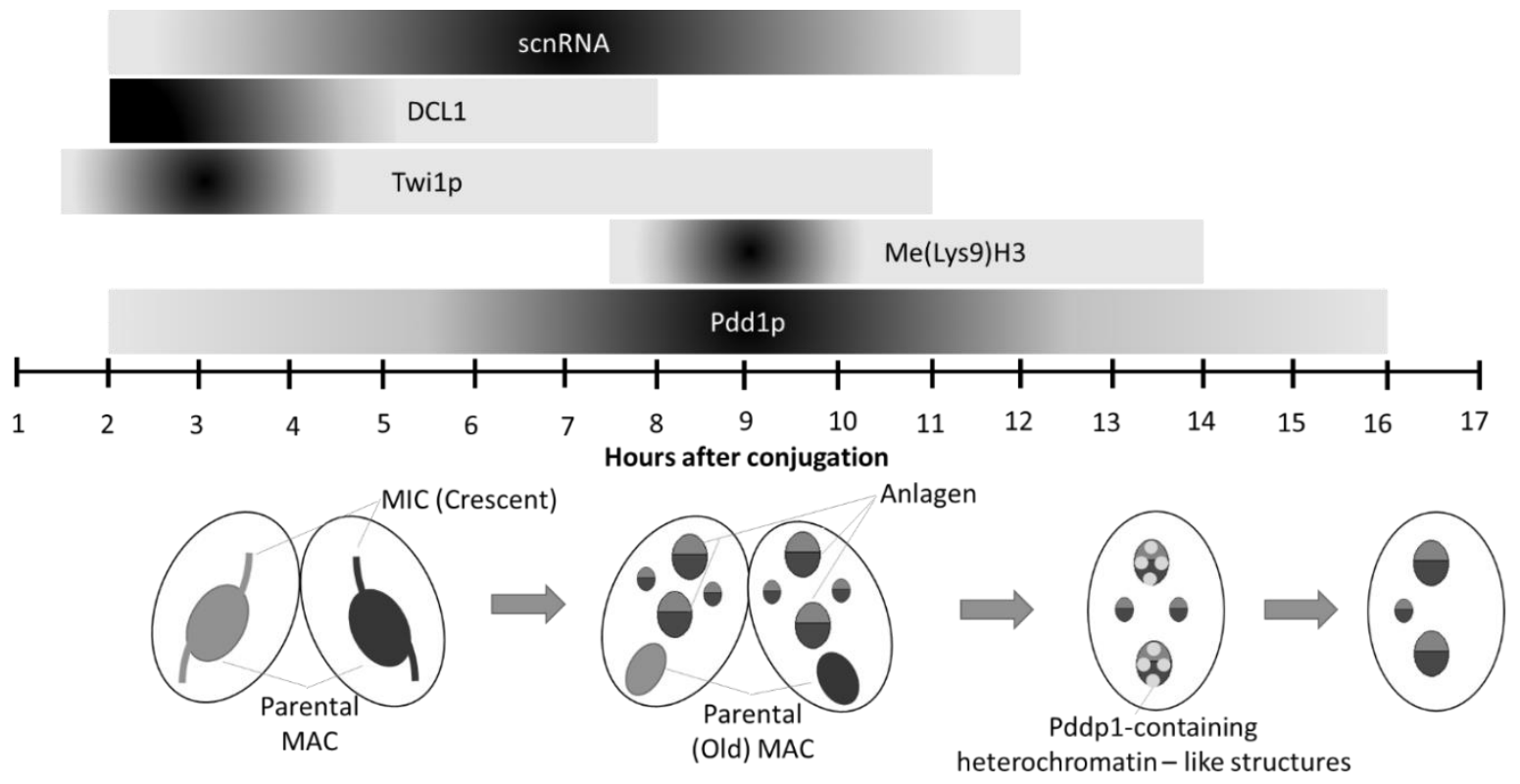

Figure 46: Intermediated molecules involved in T.thermophila's DNA rearrangement.

The darker areas of the bars represent the expression peaks. Ezlp1 tri-methylates H3K9 (Me(Lys9)H3)

\subsection{Characterization of potential ISW1-FZZ transformants during development}

The publicly available gene expression data (Figure 45) suggests Iswil is not expressed during growth. In materials and methods and in chapter 1, I described the steps for cloning of ISW1-FZZ tagging construct and generation of lines expression ISW1-FZZ. I was able to tag one strain from B2086 cells; ISWI1-FZZ B, and three from CU428 cells; ISWI1-FZZ C1, ISWI1FZZ C2 and ISWI1-FZZ C3. Starved cells of different mating types were mixed and collected at 2 and 3 hours (Figure 47), as these are the approximate times that the expression profile indicated high Iswil expression (Figure 45). I used TCA precipitation followed by Western blotting analysis to assess whether the 4 transformed pm-r cells are expressing ISWI1-FZZ during conjugation. Western blotting of the WCEs using the M2 antibody shows that only the ISWI1-FZZ strain is expressing a protein of about $150 \mathrm{kDa}$ which corresponds to $18 \mathrm{kDa}$ from the FZZ and $132 \mathrm{kDa}$ from the Iswil protein. As expected from data shown in Figure 47, ISWI1 expresses during conjugation. 


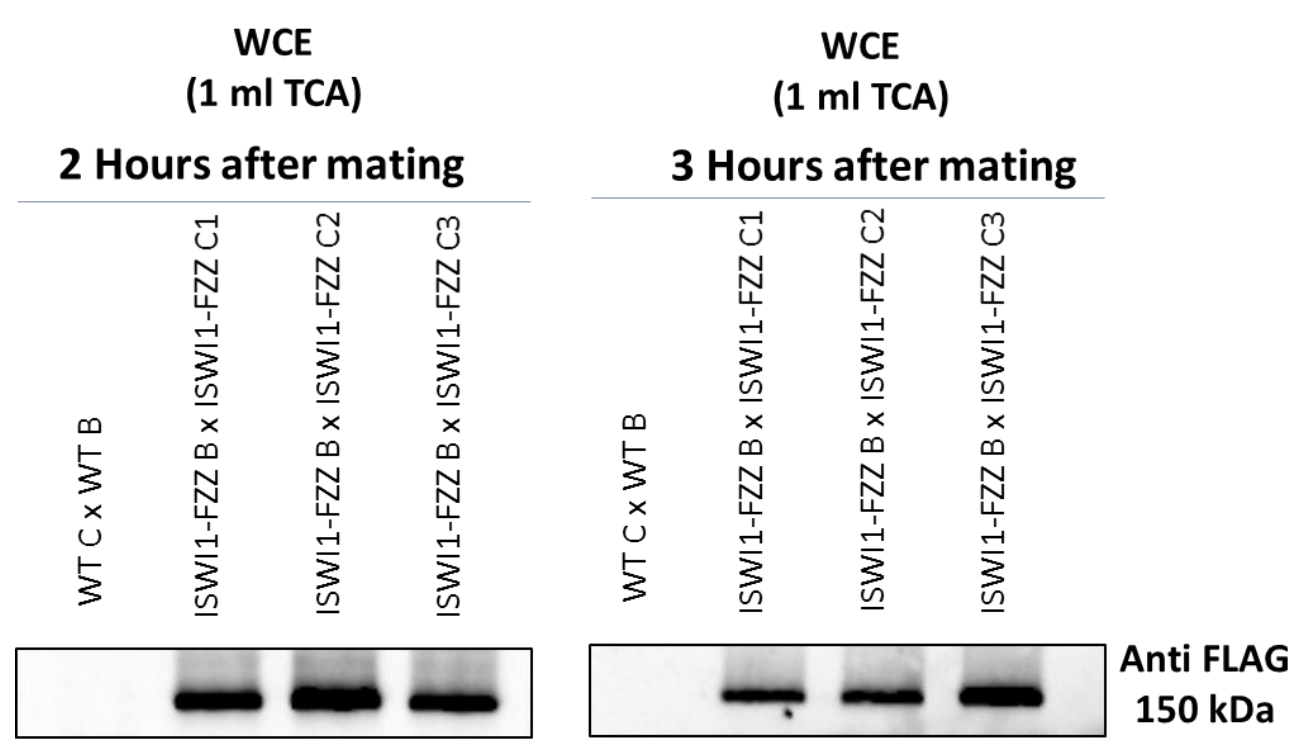

Figure 47: Characterization of potential ISW1-FZZ transformants. Iswi1 (Anti FLAG) expresses during conjugation.

\subsubsection{Expression analysis to assess whether individual strains are expressing ISWI1-FZZ during conjugation}

For the conjugations that gave a positive signal for ISW1-FZZ in Figure 47, it is impossible to tell whether one or both of the strains are actually expressing ISW1-FZZ. Therefore I mixed starved cells of each of the obtained transformants with WT cells of a different mating type and then performed a similar experiment to that described above to assess whether individual strains are expressing ISWI1-FZZ. The western blot of Figure 48 shows that every transformed cell is expressing ISWI1-FZZ. Western blotting using the M2 antibody shows that only the ISWI1-FZZ strain is expressing a protein of about 150kDa which corresponds to the ISWI1-FZZ protein. Western blotting using anti-Brg1 is used as a loading control. 


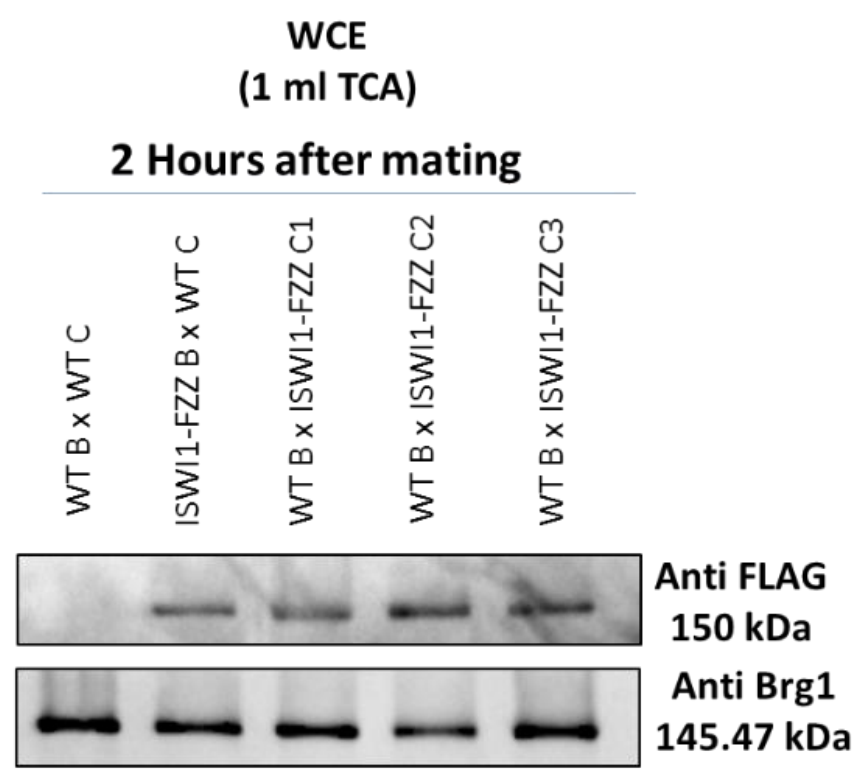

Figure 48: Expression analysis to assess whether individual strains are expressing ISWI1FZZ during conjugation.

Anti-FLAG shows that all transformants are expressing ISWI1-FZZ.

\subsection{Expression analysis of ISW1 during growth}

Although the publically available gene expression data indicates it is not expressed during growth (Figure 47), I next used TCA precipitation followed by Western blotting analysis to assess whether the two of the four transformed pm-r cells are expressing Iswi1 during vegetative growth. Unexpectedly, Iswi1 is also expressed during vegetative growth. Western

blotting using the M2 antibody shows that the ISWI1-FZZ (Figure 49) strain is expressing a protein of about $150 \mathrm{kDa}$ which corresponds to the ISWI1-FZZ protein. To further characterize ISW1 expression at the protein level confirm this, a time course experiment for ISWI1 expression throughout T.thermophila's life cycle was performed. 
WCE

(1 ml TCA)

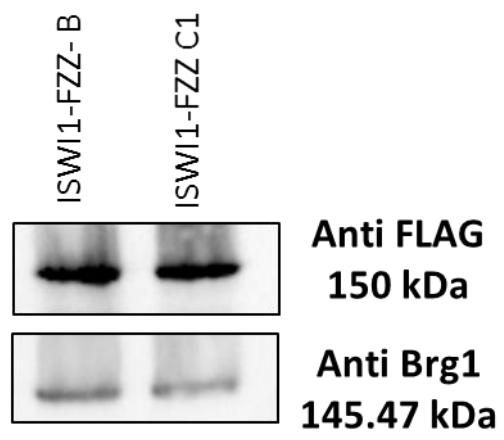

Figure 49: Expression analysis of ISWI1-FZZ during vegetative growth. Iswi1 (Anti FLAG) expresses during vegetative growth.

\subsection{Expression analysis of ISWI1-FZZ during T.thermophila's life cycle}

I next performed a time course expression analysis of Iswi1 (Figure 50). I made TCA WCEs from the indicated times in the Tetrahymena life cycle and analyzed them using SDSPAGE and Western blotting. I used anti-Brg1 antibody as a loading control for expression throughout T.thermophila's life cycle, and monitored Pdd1 expression strictly as a developmental loading control. With these results, it was evident that Iswi1 expresses throughout T.thermophila's life cycle.

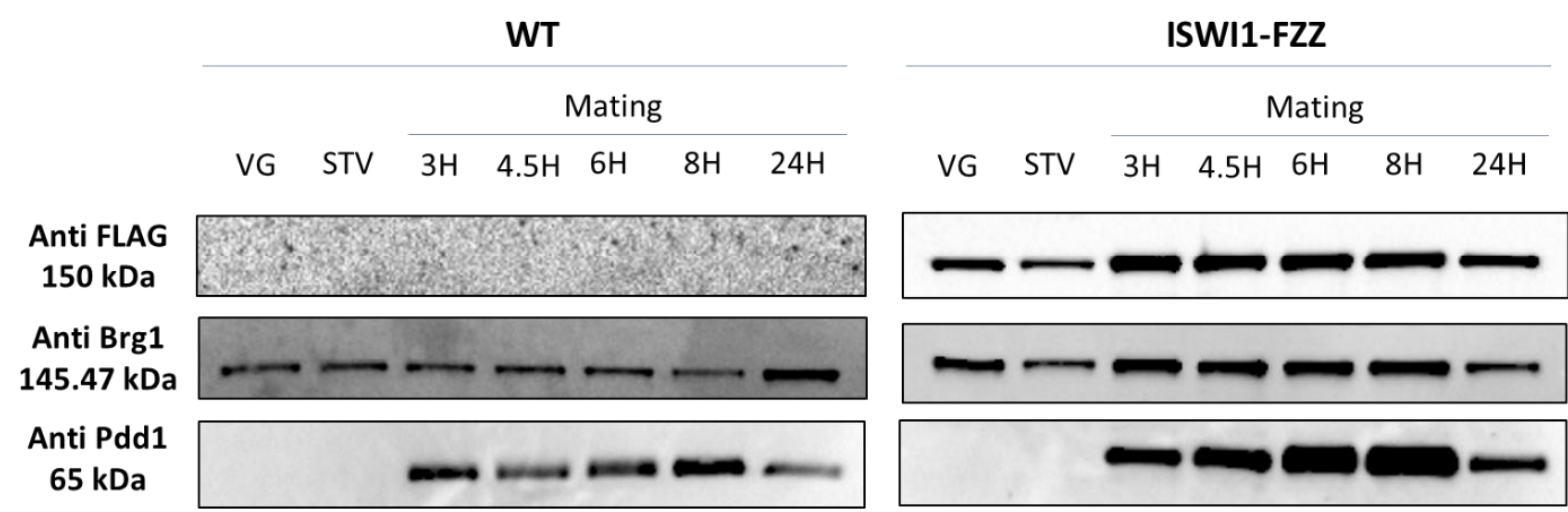

Figure 50: Expression analysis of ISWI1-FZZ during T.thermophila's life cycle Iswi1 (Anti FLAG) expresses throughout T.thermophila's life cycle. Brg1 is a loading control for expression throughout T.thermophila's life cycle and Pdd1 is strictly a developmental loading control. 


\subsection{Analysis of ISWI1-FZZ during conjugation}

In order to analyze the expression analysis of BD-FZZ during conjugation I affinity purified the FZZ-tagged protein and any co-purifying proteins, I performed one-step affinity chromatography on whole cell extracts made from cells in conjugation expressing ISWI1-FZZ. For the Input, the M2 antibody Figure 51 shows that only the ISWI-FZZ strains are expressing a protein of about 150kDa corresponding to ISWI1-FZZ. For the IP, the M2 antibody Figure 51 shows that only the ISWI1-FZZ strain is expressing a protein of about $150 \mathrm{kDa}$ corresponding to ISWI1-FZZ. WT represents the mock IP.

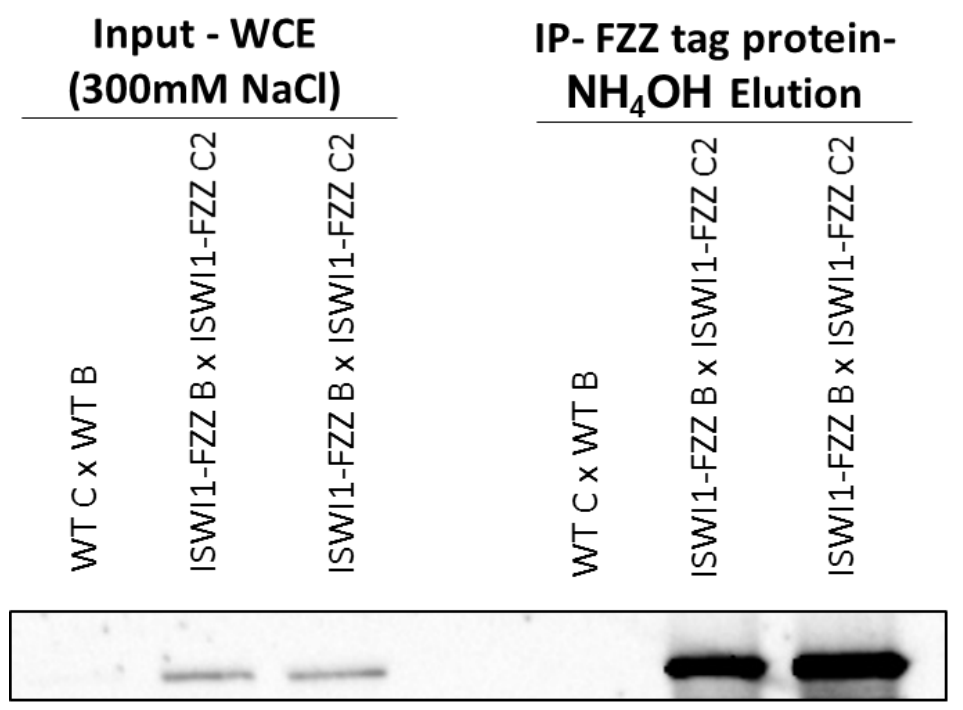

\section{Anti FLAG}

$150 \mathrm{kDa}$

Figure 51: Expression analysis of ISWI1-FZZ during conjugation Iswi1 (Anti FLAG) expresses during conjugation

\subsection{MS and SAINTexpress analysis of ISWI1-FZZ during conjugation}

Through SAINTexpress analysis of the mass spectrometry data from the Iswi1 affinity purification, I have identified several potential interacting proteins at this point it is not known if they are related to DNA rearrangements. 
Table 10: SAINTexpress-curated data for 2 replicates of ISWI1-FZZ during conjugation

\begin{tabular}{|l|l|l|}
\hline \multicolumn{1}{|c|}{ Protein } & Gene Name & SpecSum \\
\hline Iswi1 & TTHERM_00388250 & 57 \\
\hline hypothetical protein & TTHERM_00247110 & 28 \\
\hline hypothetical protein & TTHERM_00582070 & 19 \\
\hline Sm protein (spliceosome) & TTHERM_01403810 & 7 \\
\hline hypothetical protein & TTHERM_00709740 & 6 \\
\hline RPT6 (26S proteasome regulatory subunit T6) & TTHERM_00551090 & 4 \\
\hline
\end{tabular}

The hypothetical protein (TTHERM_00247110) has a similar (Figure 45) gene expression profile to Iswi1 (Figure 52) therefore they may be working together. This makes this protein an excellent candidate for tagging and further analysis. In addition, this protein does not have known domains (NCBI).

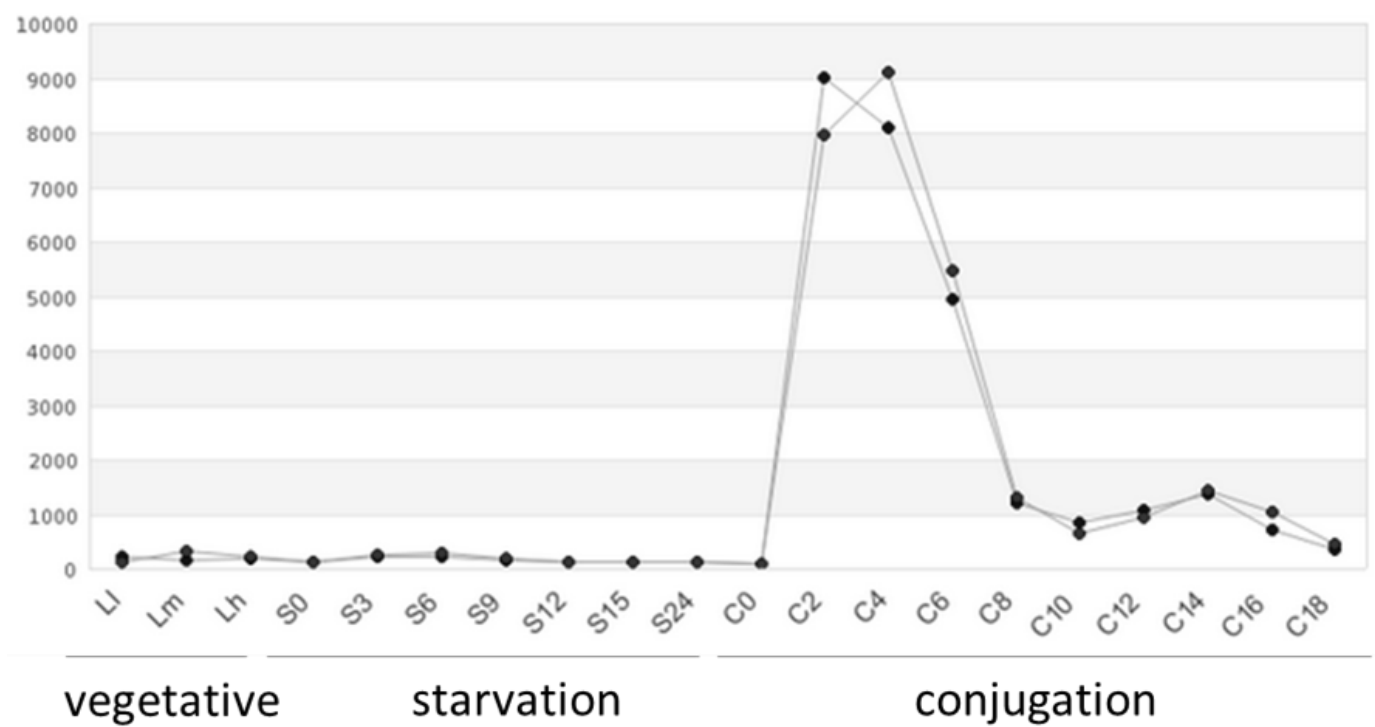

Figure 52: TTHERM_00247110 gene expression profile from RNA-seq

Tetrahymena functional genomics database from ciliate.org 


\subsection{Iswi1 localizes to the MIC during growth and sexual development including meiosis}

In order to further characterize the function of Iswi1, I performed IF. I found that Iswi1 localizes to the MIC during growth and sexual development, including meiosis. Localization of Iswil protein to the MIC is consistent with role in mitosis, or maintenance of silent chromatin (Figure 53). Iswi1 does not localize to where other proteins (Pdd1p, Twi1p, Ezlp1 and DCL1) related to DNA rearrangement localize. Therefore Iswil localization to MIC might mean that it is involved in transcription of MIC long ncRNAs that are the precursors to scnRNA. 


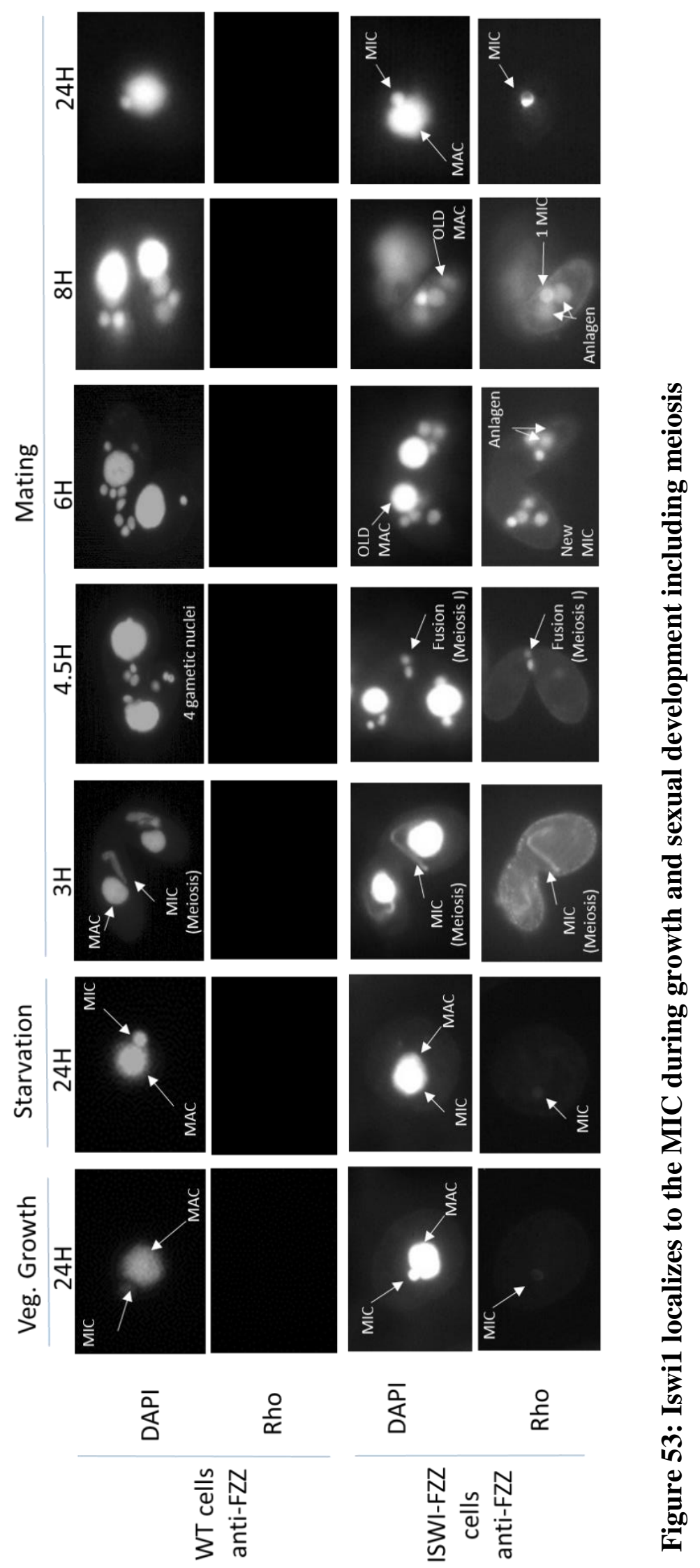




\subsection{Model of ISWI1 localization during T.thermophila's life cycle}

Figure 54 shows that Iswil localizes to the MIC during vegetative growth, starvation, meiosis $(3 \mathrm{H})$, gametic nuclei fusion $(4.5 \mathrm{H})$ and zygotic nuclei duplication $(6 \mathrm{H})$. At $8 \mathrm{H}$ during anlagen development, Iswi1 localizes to anlagen (new MAC) and new MIC. This is consistent with a role in mitosis, maintenance of silent chromatin, perhaps transcription of some specific genes during meiosis, gametic differentiation and fusion, and development of the new MIC and MAC. At $24 \mathrm{H}$ when the daughter cells have formed, the expressed Iswil from the parental MAC might be stable enough to localize to the MIC of the daughter cells.

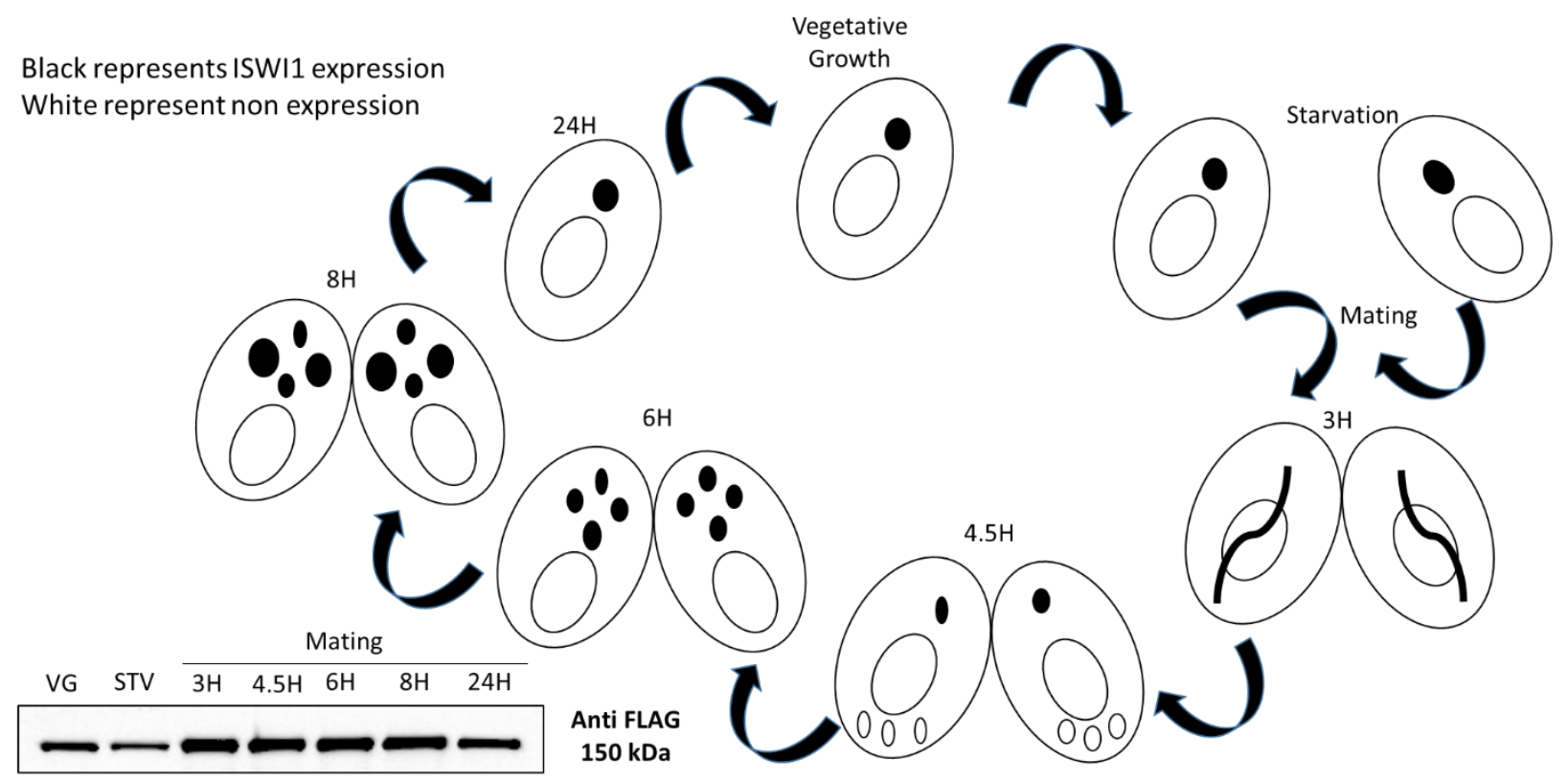

Figure 54: Model of ISWI1 localization during T.thermophila's life cycle 


\section{SUMMARY AND FUTURE DIRECTIONS}

\subsection{SWI/SNF complex}

AP-MS of tagged TtSnf5 revealed members of a putative TtSWI/SNF complex. One of these revealed members was a bromodomain containing protein (Ibd1). This protein was selected and tagged using molecular cloning methods. The purpose of tagging Ibd1 was to confirm reciprocal AP-MS interactions with Snf5. The rationale behind reciprocal purifications is to validate the identity of the SWI/SNF complex and possibly identify new members. Through APMS of Tetrahymena Snf5, and Snf5-interacting Ibd1, I have identified the first protist SWI/SNF complex. In addition, perhaps Tetrahymena SWI/SNF has two proteins that potentially recognize PTM on histones, the PHD domain (TTHERM_00241840) and Ibd1 (TTHERM_00729230). These two proteins could work in conjunction to positively regulate transcription at an increased rate. One possible double PTM recognized by SWI/SNF could be chromatin tri-methylated at H3K4 (Taverna et al., 2007) and multiple acetylations on H4 which is correlated with highly transcribed genes (Turner et al., 1992). A ciliate-specific protein (TTHERM_00092790) was found and for this protein it could be established if the expression of the gene encoding this protein is essential for growth. If the protein is essential it can be used as a target protein for a rational design drug of ciliate specific antibiotic. For example this drug could be used against the parasitic ciliate Ichthyophthirius multifiliis which affects aquiculture (Sigh et al., 2004).

Fillingham et al. (Fillingham et al., 2006) showed that TtBrg1 localizes to the MAC during growth and development. This is consistent with a role in transcription during growth. IF for SWI/SNF showed localization of Ibd1 protein and H4K8ac to MAC consistent with Fillingham's results. To further illustrate the SWI/SNF member's localization, IF of Snf5 can be performed. Our data for Ibd1 localization suggests that Ibd1 localizes to the MAC during vegetative growth, starvation, meiosis $(3 \mathrm{H})$, gametic nuclei fusion $(4.5 \mathrm{H})$ and zygotic nuclei duplication $(6 \mathrm{H})$. At $8 \mathrm{H}$ during anlagen development, Ibd1 localizes to the anlagen (new MAC) and old MAC. At this point the anlagen or new MAC takes over transcription from the old MAC which begins to degrade. Since Brg1 (Fillingham et al (2006) and Ibd1 (this study) follow this pattern we hypothesize that Ibd1 and SWI/SNF have a role in transcription. 


\subsubsection{Ibd1}

AP-MS of Ibd1 during vegetative growth suggests that in addition to being a member of SWI/SNF in Tetrahymena, (Table 5). Ibd1 interacts with multiple proteins (SWR, HMT, SAGA) that have not been yet studied in Tetrahymena. These Ibd1 interacting proteins correspond to ortologues that are part of known transcription-related complexes in yeast and humans. Our data suggests that during conjugation, there is a change in the complexes that Ibd1 is interacting with. However, my analysis shows that 5 hours after the initiation of conjugation, when the zygotic nucleus is being formed by gametic nuclei fusion, Ibd1 still interacts with the same complexes but the composition is different (Table 9). Figure 23 shows that at 5 hours after conjugation there is a decrease in the amount of Ibd1 that is interacting with all SWI/SNF subunits and the HMT (TTHERM_00486440). However, there is an increase relative to SWI/SNF and HMT (TTHERM_00486440) of all putative SWR-C and putative SAGA subunits present in the Ibd1 affinity purification in conjugation. Perhaps Ibd1 has an important role in histone variant exchange and histone acetylation during gametic nuclei fusion. Perhaps Ibd1's function as a member of the SWI/SNF complex or HMT is not required. Interestingly, the hypothetical protein (TTHERM_00046150), along with other proteins present in this purification, such as PHD Finger (TTHERM_00444700), AT-hook containing (TTHERM_00355040) and ciliate-specific (TTHERM_00136450), drastically increase in the percentage interacting with Ibd1 at this stage during conjugation. These last mentioned proteins might be also important for gametic nuclei fusion along with putative SWR-C and putative SAGA.

An interesting question that arose from this research is if during vegetative growth and conjugation all of the Ibd1 related complexes work independently or as a mega complex. This question was based on the fact that Tetrahymena thermophila is a large unicellular organism $(\sim 50 \mu \mathrm{m})$ and having a high rate of transcription is of paramount importance for maintenance. The first model supported by this study is during vegetative growth and suggests that highly transcribed regions have multiple acetylations in $\mathrm{H} 4$. These regions are recognized by Ibd1 to recruit SWI/SNF, SWR, SAGA and HMT all of which work together to form a mega complex. This mega complex will increase the transcription rate on a subset of genes during vegetative 
growth. The second model suggest that during conjugation the complexes work independently. The comparative analysis of both AP-MS data from vegetative growth and 5 hours after mating (Figure 23) shows that the number of subunits of each complex is being drastically modified during conjugation compared to vegetative growth. Perhaps during conjugation these complexes are related to chromatin assembly and not to increased transcription rate. In order to determine if the mentioned complexes work independently or as a mega complex in these two physiological states, AP-MS experiments of SAGA, SWRC, HMT members are needed. Alternatively, a gel filtration of Ibd1 to determine the number of distinct Ibd1-containing protein complexes is needed. For the gel filtration of Ibd1, first I would follow all the mentioned steps to perform an AP in materials and methods. However, instead of using a denaturing elution presented in this work I would use a native elution (3xFLAG peptide) to conserve the complex's composition. The 3xFLAG peptide competes with the FZZ-tagged protein for the M2 matrix. After I have the eluates with the native composition of the complexes I will gel filtrate them to separate them by size and then I will analyse each fraction by MS followed by SAINTexpress as described in materials and methods. With this data I will be able to determine the complexes composition.

Another important question raised by this work is whether Ibd1's bromodomain is functional since Brg1 lacks a BD. Based on this my next objective was to answer if Ibd1 is functional. To determine Ibd1's functionality, I engineered a synthetic Ibd1 gene to express a recombinant 6xHis-Ibd1 in E.coli BL21 (D3). The purified recombinant protein was used in an array containing sites with single PTM and combinations of PTM. The peptide array confirms that Ibd1 is functional in that it recognizes the canonical target of bromodomains and suggests a role in transcription. My data is consistent with Ibd1 recognizing combinations of marks rather than singly acetylated residues. As suggested in the literature (Filippakopoulos et al., 2012) bromodomains may recognize acetylations and phosphorylations, suggesting that some bromodomains recognize combinations of marks rather than singly acetylated residues. The data obtained from the peptide array suggest that Ibd1 better recognizes multiple peptides. The best candidate combinations to be recognized by Ibd1 as suggested by the peptide array are TtH4K4ac, TtH4K7ac, TtH4K11ac, TtH4K15ac and TtH4K4ac, TtH4K7ac, TtH4K11ac. These PTM are related to highly transcribed regions (Turner et al., 1992). To determine the exact combination of PTM recognized by Ibd1, a dissociation constant experiment can be performed 
since it is possible to measure the binding constants $\left(\mathrm{K}_{\mathrm{d}}\right)$ that Ibd1 may have with the different candidate PTM as described in the literature (Taverna et al., 2002). Once the PTM are determined we could use ChIP-Seq to identify the Ibd1 binding sites in the genome during growth. This experiment will be followed by ChIP-Seq with the antibodies against the PTM given by the $K_{d}$ experiment. Thus, with these two results we will determine if Ibd1 and the PTM are found in the same genomic region corresponding to highly transcribed regions.

Some other questions raised by this work are: 1] is the SWI/SNF PHD domain (TTHERM_00241840) functional. In order to answer this question a recombinant protein can be engineered and the peptide array experiment can be used. 2] Tetrahymena thermophila has 12 bromodomain containing proteins including Gen5 and Ibd1. From the 12 bromodomain containing proteins, one protein (TTHERM_00483490) has a very similar structure to Ibd1 and characterizing this protein could be interesting.

\subsection{ISWI complex}

I initially hypothesized that Iswi1 is involved in meiosis/DNA rearrangements. I based this hypothesis on the publically available gene expression profile for Iswil. Iswil is highly expressed at 2 and 7 hours during conjugation, which coincides with the time during conjugation where other proteins involved in DNA rearrangements are being expressed and I thought Iswi1 may have been the protein that regulates the expression of the scnRNA precursor or long RNA. To test this hypothesis, I tagged the Iswi1 protein (TTHERM_00388250). Unexpectedly, Iswi1 also expresses during vegetative growth. I confirmed this through a time course experiment for ISWI1 expression as assessed by western blotting throughout Tetrahymena's life cycle was performed. Through SAINTexpress analysis of AP-MS data of Iswi1-FZZ I have identified several potential interacting proteins, at this point it is not known if they are related to DNA rearrangements. Significantly, the hypothetical protein (TTHERM_00247110) has a similar gene expression profile to Iswi1 making this protein an excellent candidate for tagging and further analysis. 
In order to further characterize the function of Iswi1, I performed IF. I found that Iswi1 localizes to the MIC during growth and sexual development, including meiosis. Localization of Iswi1 protein to the MIC is consistent with role in mitosis, or maintenance of silent chromatin (Figure 53). Iswi1 does not localize to where other proteins (Pdd1p, Twi1p, Ezlp1 and DCL1) related to DNA rearrangement localize. Therefore Iswil localization to MIC might mean that it is involved in transcription of MIC long ncRNAs that are the precursors to scnRNA. Our model for Iswi1 localization suggests that Iswi1 localizes to the MIC during vegetative growth, starvation, meiosis $(3 \mathrm{H})$, gametic nuclei fusion $(4.5 \mathrm{H})$ and zygotic nuclei duplication $(6 \mathrm{H})$. At $8 \mathrm{H}$ during anlagen development,

Some of the questions raised by this work are: 1] what is the function of ISWI1 in Tetrahymena? 2] What is the phenotypic analysis of MIC integrity in ISWI1-KO in growth and meiosis? 3] What is the function of ISWI1 interacting proteins - are they specific for development? 


\section{APPENDICES}

\subsection{Appendix 1 - Recipes}

\begin{tabular}{|c|c|}
\hline Reagent & Composition \\
\hline $0.8 \%$ Agarose Gel $(\mathrm{w} / \mathrm{v})(50 \mathrm{~mL})$ & $\begin{array}{l}0.4 \mathrm{~g} \text { agarose } \\
50 \mathrm{~mL} 1 \mathrm{xTBE} \text { or } 1 \mathrm{xTAE} \\
5 \mu \mathrm{L} \text { RedSafe Nucleic Acid Stain, FroggaBio }\end{array}$ \\
\hline 0.5M Ammonium Hydroxide $\left(\mathrm{NH}_{4} \mathrm{OH}\right)$ & 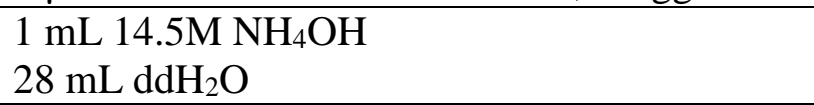 \\
\hline $10 \%$ APS (Ammonium Persulfate) (w/v) & 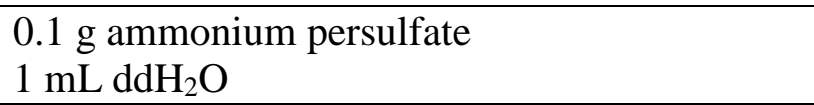 \\
\hline $1 \mathrm{M} \mathrm{CaCl}_{2}(1 \mathrm{~L})$ & $\begin{array}{l}\mathrm{CaCl}_{2}=110.99 \mathrm{~g} / \mathrm{mol} \\
1 \mathrm{~mole} \mathrm{CaCl}_{2}: 1 \mathrm{~L} \mathrm{ddH} \mathrm{dd}_{2} \mathrm{O}\end{array}$ \\
\hline $2 \mathrm{mM} \mathrm{CaCl}_{2} / 20 \mathrm{mM}$ Tris & $\begin{array}{l}100 \mu \mathrm{L} 1 \mathrm{M} \mathrm{CaCl}_{2} \\
1 \mathrm{~mL} 1 \mathrm{M} \text { Tris pH } 8.0 \\
48.9 \mathrm{~mL} \mathrm{ddH_{2 } \mathrm { O }}\end{array}$ \\
\hline $\begin{array}{l}\text { 0.5M EDTA, Iron (III) Sodium Salt } \mathrm{pH} 8.0 \\
(500 \mathrm{~mL})\end{array}$ & $\begin{array}{l}91.78 \mathrm{~g} \mathrm{Na} 2 \text { EDTA }(367.1 \mathrm{~g} / \mathrm{mol}) \\
\text { ddH2O up to } 500 \mathrm{~mL} \text {, adjust pH to } 8.0 \text { using } \\
\mathrm{NaOH}(1 \mathrm{~N})\end{array}$ \\
\hline $\begin{array}{l}\text { 2x Lysis Buffer }(50 \mathrm{~mL}) \\
\text { Note: For } 1 \mathrm{x} \text { Lysis } \mathrm{Buffer} \text {, prepare equal parts } \\
\text { 2x Lysis Buffer and } \mathrm{ddH}_{2} \mathrm{O}\end{array}$ & $\begin{array}{l}2 \mathrm{~mL} 1 \mathrm{M} \text { Tris } \mathrm{pH} 8.0 \\
50 \mu \mathrm{L} 1 \mathrm{M} \mathrm{MgCl}_{2} \\
42 \mathrm{~mL} \mathrm{ddH} \mathrm{O}_{2} \mathrm{O} \\
6 \mathrm{~mL} 5 \mathrm{M} \mathrm{NaCl} \\
500 \mu \mathrm{L} \text { Protease Inhibitor Cocktail, Sigma } \\
200 \mu \mathrm{L} \text { phenylmethylsulfonyl fluoride (PMSF) }\end{array}$ \\
\hline $1 \mathrm{M} \mathrm{MgCl}_{2}(203.3 \mathrm{~g} / \mathrm{mol})(100 \mathrm{~mL})$ & $20.33 \mathrm{~g} \mathrm{MgCl}_{2} ; \mathrm{ddH}_{2} \mathrm{O}$ to $100 \mathrm{~mL}$ \\
\hline $5 \times \mathrm{TBS}$ & $\begin{array}{l}48.44 \mathrm{~g} \mathrm{Tris} \\
584.4 \mathrm{~g} \mathrm{NaCl} \\
3.5 \mathrm{~L} \mathrm{ddH} \mathrm{dd}_{2} \mathrm{O} \\
\text { Adjust pH to } 7.5 \text { using } \mathrm{HCl} \\
\text { Add } \mathrm{ddH}_{2} \mathrm{O} \text { up to } 4 \mathrm{~L}\end{array}$ \\
\hline $3 \%$ Milk (50 mL) & $\begin{array}{l}1.5 \mathrm{~g} \text { dried milk powder } \\
50 \mathrm{~mL} 1 \mathrm{xTBS}\end{array}$ \\
\hline 10x Western Buffer & $\begin{array}{l}121.2 \mathrm{~g} \text { Tris } \\
576 \mathrm{~g} \text { glycine } \\
\text { Add up to } 4 \mathrm{~L} \text { of } \mathrm{ddH}_{2} \mathrm{O}\end{array}$ \\
\hline 1x Running Buffer & $\begin{array}{l}100 \mathrm{~mL} 10 x \text { Western Buffer } \\
900 \mathrm{~mL} \mathrm{ddH} \mathrm{H}_{2} \mathrm{O} \\
10 \mathrm{~mL} 10 \% \mathrm{SDS}\end{array}$ \\
\hline $10 \% \operatorname{SDS}(\mathrm{w} / \mathrm{v})$ & 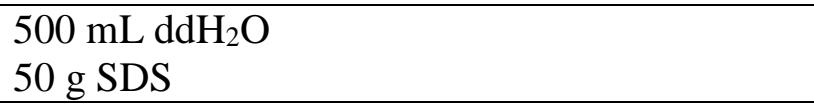 \\
\hline 100mM NaCl Wash Buffer (IPP100) & $\begin{array}{l}500 \mu \mathrm{L} 1 \mathrm{M} \text { Tris } \mathrm{pH} 8.0 \\
1 \mathrm{~mL} 5 \mathrm{M} \mathrm{NaCl} \\
500 \mu \mathrm{L} 10 \% \mathrm{NP}-40\end{array}$ \\
\hline
\end{tabular}




\begin{tabular}{|c|c|}
\hline & $48 \mathrm{~mL} \mathrm{ddH_{2 } \mathrm { O }}$ \\
\hline 300mM NaCl Wash Buffer (IPP300) & 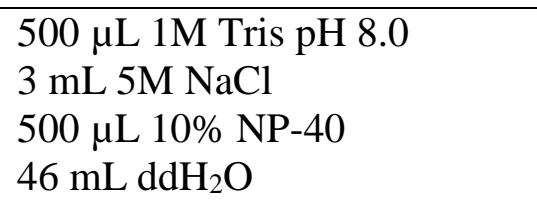 \\
\hline $5 \mathrm{M} \mathrm{NaCl}(500 \mathrm{~mL})$ & $\begin{array}{l}146.1 \mathrm{~g} \mathrm{NaCl} \\
\mathrm{ddH}_{2} \mathrm{O} \text { up to } 500 \mathrm{~mL}\end{array}$ \\
\hline $10 \%$ NP-40 (v/v) & $\begin{array}{l}2.5 \mathrm{~mL} \mathrm{NP}-40 \\
22.5 \mathrm{ddH}_{2} \mathrm{O}\end{array}$ \\
\hline 10xPBS pH 7.3 (1L) & $\begin{array}{l}82 \mathrm{~g} \mathrm{NaCl} \\
2.64 \mathrm{~g} \mathrm{NaH}_{2} \mathrm{PO}_{4} \\
16 \mathrm{~g} \mathrm{Na}_{2} \mathrm{HPO}_{4} \\
\mathrm{ddH}_{2} \mathrm{O} \text { up to } 1 \mathrm{~L}, \mathrm{pH} 7.3\end{array}$ \\
\hline 1xPBST (500 mL) & $\begin{array}{l}500 \mathrm{~mL} 1 \mathrm{xPBS} \\
250 \mu \mathrm{L} \text { Tween } 20\end{array}$ \\
\hline 100mM PMSF (10 mL) & $\begin{array}{l}0.1742 \text { g PMSF } \\
10 \mathrm{~mL} \text { isopropanol }\end{array}$ \\
\hline Ponceau $(0.1 \% \mathrm{w} / \mathrm{v})(1 \mathrm{~L})$ & $\begin{array}{l}1 \mathrm{~g} \text { Ponceau } \mathrm{S} \\
50 \mathrm{~mL} \text { acetic acid } \\
\mathrm{ddH}_{2} \mathrm{O} \text { up to } 1 \mathrm{~L}\end{array}$ \\
\hline 2xSDS Loading Dye & $\begin{array}{l}1.15 \mathrm{~mL} 10 \% \text { SDS } \\
37.5 \mu \mathrm{L} 2 \mathrm{M} \text { Tris, } \mathrm{pH} 6.8 \\
62.5 \mu \mathrm{L} \text { beta-mercaptoethanol } \\
\text { Pinch of bromophenol blue }\end{array}$ \\
\hline SPP Media (4 L) & $\begin{array}{l}40 \mathrm{~g} \text { protease peptone } \\
4 \mathrm{~g} \text { yeast extract } \\
8 \mathrm{~g} \text { glucose } \\
0.12 \mathrm{~g} \text { Sequestrine, Sigma } \\
\mathrm{dd}_{2} \mathrm{O} \text { up to } 4 \mathrm{~L}\end{array}$ \\
\hline Tetrahymena Lysis Solution $(500 \mathrm{~mL})$ & $\begin{array}{l}210 \mathrm{~g} \text { urea } \\
35 \mathrm{~mL} 5 \mathrm{M} \mathrm{NaCl} \\
5 \mathrm{~mL} 1 \mathrm{M} \text { Tris, pH } 7.4 \\
10 \mathrm{~mL} 0.5 \mathrm{M} \text { EDTA } \\
50 \mathrm{~mL} 10 \% \text { SDS } \\
\text { dd }_{2} \mathrm{O} \text { up to } 500 \mathrm{~mL} \\
\end{array}$ \\
\hline 1xTEV Cleavage Buffer & $\begin{array}{l}500 \mu \mathrm{L} 1 \mathrm{M} \mathrm{Tris}, \mathrm{pH} 8.0 \\
1 \mathrm{~mL} 5 \mathrm{M} \mathrm{NaCl} \\
500 \mu \mathrm{L} 10 \% \mathrm{NP}-40 \\
50 \mu \mathrm{L} 0.5 \mathrm{M} \mathrm{EDTA} \\
48 \mathrm{~mL} \mathrm{ddH}_{2} \mathrm{O}\end{array}$ \\
\hline 10mM Tris, $\mathrm{pH} 7.4(1 \mathrm{~L})$ & $\begin{array}{l}1.21 \mathrm{~g} \text { Tris } \\
\mathrm{ddH}_{2} \mathrm{O} \text { up to } 1 \mathrm{~L}, \mathrm{pH} 7.4\end{array}$ \\
\hline LB Liquid Media & $\begin{array}{l}1 \mathrm{~L} \mathrm{ddH} \mathrm{H}_{2} \mathrm{O} \\
25 \mathrm{~g} \mathrm{LB}, \text { Miller }\end{array}$ \\
\hline LB Media for Plates & $\begin{array}{l}1 \mathrm{~L} \mathrm{ddH} 2 \mathrm{O} \\
25 \mathrm{~g} \mathrm{LB}, \text { Miller }\end{array}$ \\
\hline
\end{tabular}




\begin{tabular}{|l|l|}
\hline & $15 \mathrm{~g}$ agar \\
\hline Running Gel (10\%) & $10 \mathrm{~mL} 1.5 \mathrm{M}$ Tris, $\mathrm{pH} 8.8$ \\
& $13 \mathrm{~mL} 30 \%$ Bis/Acrylamide, 37.5:1 \\
& $17 \mathrm{~mL}$ ddH2O \\
& $40 \mu \mathrm{L}$ Temed \\
& $200 \mu \mathrm{L} 10 \%$ APS \\
\hline Stacking Gel (4\%) & $3.8 \mathrm{~mL} 0.5 \mathrm{M}$ Tris, $\mathrm{pH} 6.8$ \\
& $2.0 \mathrm{~mL} 30 \%$ Bis/Acrylamide, 37.5:1 \\
& $9.0 \mathrm{~mL} \mathrm{ddH} \mathrm{d}_{2} \mathrm{O}$ \\
& $15 \mu \mathrm{L}$ Temed \\
& $75 \mu \mathrm{L} 10 \%$ APS \\
\hline Primary Antibody Buffer $(50 \mathrm{~mL})$ & $1.5 \mathrm{~g}$ albumin \\
& $50 \mathrm{~mL} 1$ xTBS \\
& $12.5 \mu \mathrm{L}$ Tween 20 \\
\hline Shandin & $100 \mathrm{~mL}$ ethanol \\
& $200 \mathrm{~mL} \mathrm{HgCl} 2$ \\
\hline
\end{tabular}




\subsection{Appendix 2 - Analysis}

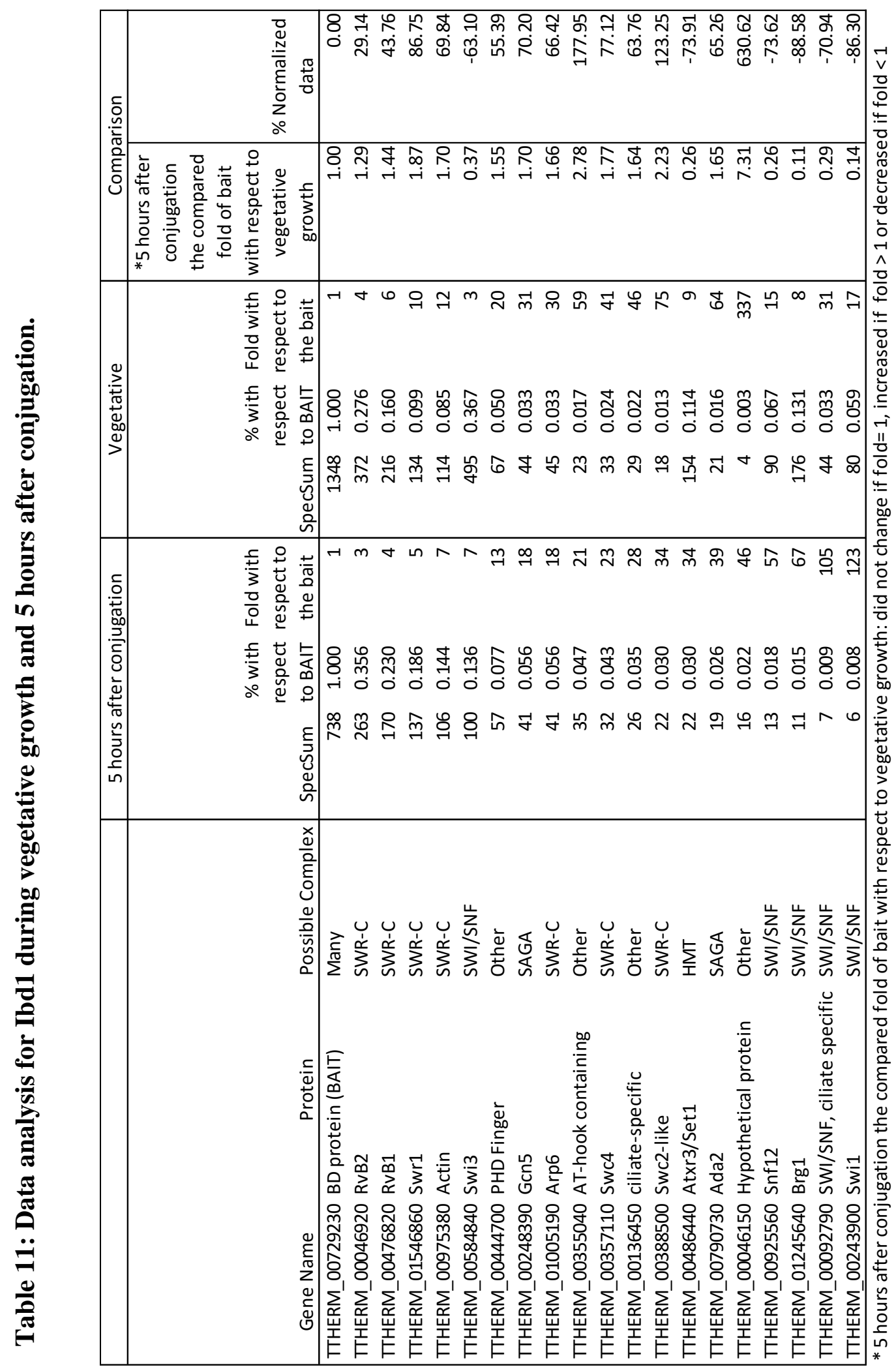




\subsection{Appendix 3 - Primers}

\subsubsection{Sequencing primers}

M13F 5'-tgtaaaacgacggccagt-3'

M13R 5'-caggaaaacagctatgac-3'

HN111 5'-tatcatcatcatctttgtaatcaaatatc-3'

\subsubsection{Bd containing protein (TTHERM_00729230) primers}

Restriction sites are underlined

7.3.2.1 Up stream TGA codon

FWR KpnI 5'-ccccGGTACCaaataatttttgaatgtattttggttttat-3'

RVR XhoI 5'-ccccCTCGAGaatcttcactctctttgtagtagtttcagt-3'

7.3.2.2 Down stream TGA codon

FWR NotI 5'-ccccGCGGCCGCagtagaaagaagaaattttaaagaagcttg-3'

RVR SacI 5'-ccccGAGCTCatatttctgctcataatatacaaactaaag-3'

\subsubsection{ISWI1 (TTHERM_00388250) primers}

\subsubsection{Up stream TGA codon}

FWR KpnI 5'-ccccGGTACCagagctttctggtaaagaggag-3'

RVR XhoI 5'-ccccCTCGAGctttttaactattttatcaattttactaaaat-3' 
7.3.3.2 Down stream TGA codon

FWR NotI 5'-ccccGCGGCCGCtttattatgctctcaaaaaagtttta-3'

RVR SacI 5'-ccccGAGCTCatagttatagctaaatttcttgtttgatta-3'

\subsubsection{Synthetic Ibd1 gene primers}

FWR NdeI 5'-ggggCATATGatgtctggtttcaagtcttcctga-3'

RVR BamHI 5'-ggggGGATCCttaaattttaacgcgcttcgtcg-3' 
7.4 Appendix 4-Protein ladder

PiNK Plus Prestained Protein Ladder

(FroggaBio)

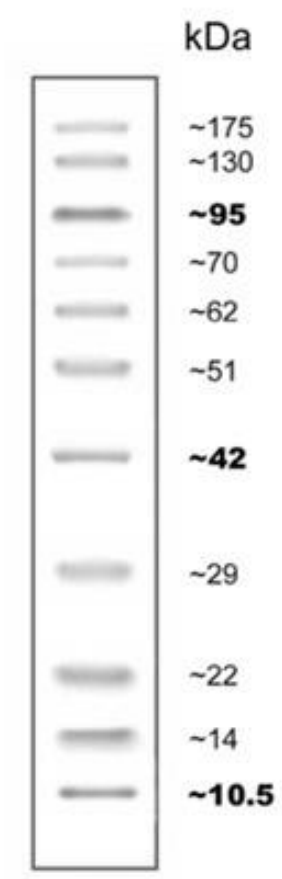

Tris-Glycine $15 \%$
BLUeye Prestained Protein Ladder

(FroggaBio)

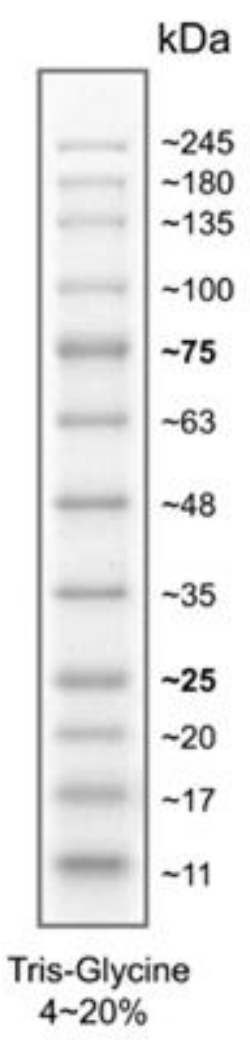




\subsection{Appendix 5 - Peptide Array}

\section{Example analysis of positive control G9a protein}

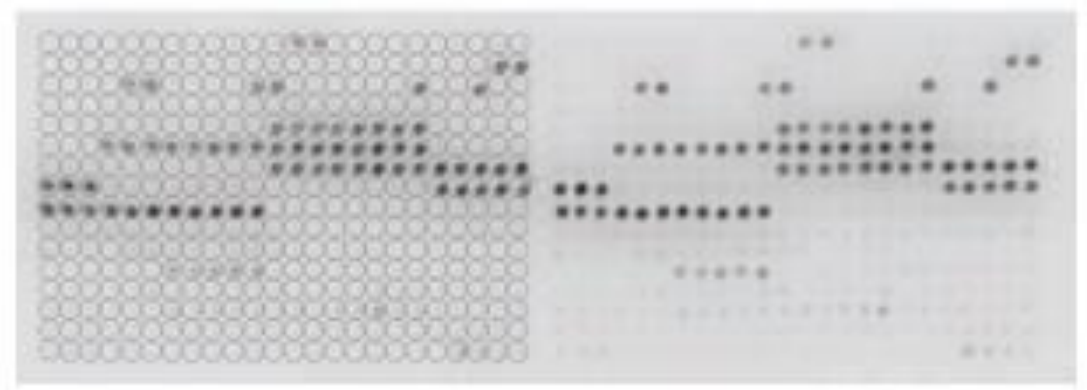

Image of ECL detection of positive control Recombinant His-G9a protein.

The positive control His-G9a protein was incubated with the MODified Histone Peptide array in combination with a c-Myc antibody (Active Motif Catalog No. 39502) for control peptide P21 according to the MODified Histone Protein Domain Binding manual at a concentration of $10 \mathrm{nM}$ for 2 hours. The anti-His6-tag antibody and $\mathrm{c}-\mathrm{Myc}$ antibody were each used at a 16,000 dilution, followed by incubation with the anti-mouse HRP-conjugated antibody at a 1:2,500 dilution and ECL detection. The chemiluminescent signal from the array was captured with a CCD camera. The image shows the grid overlay from the Array Analyze software for spot identification on the left hand side of the array image.

\section{G9a Specificity Analysis (Multiple Peptide Average)}

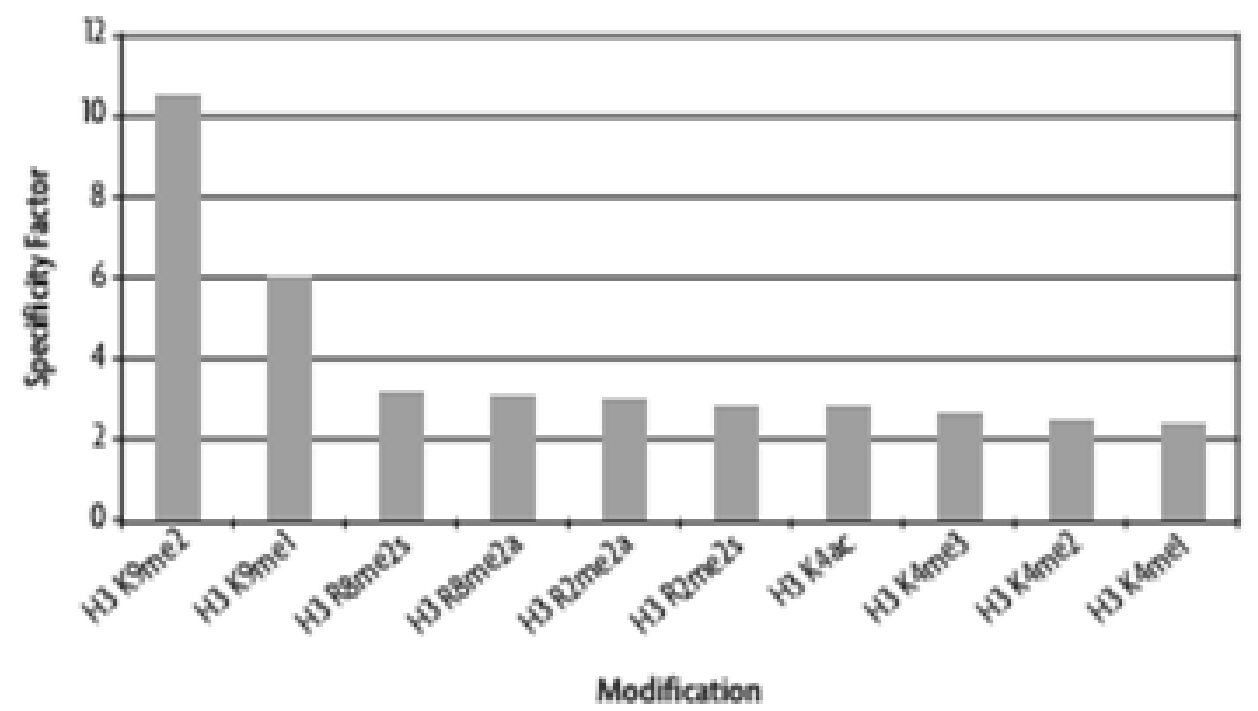

Graphical analysis of the positive control G9a protein.

Active Motif's Array Analyze Software was used to analyze spot intensity from the chemiluminescent image. The results were graphed as a specificity factoc, which is the ratio of the average intensity of all spots containing the modification divided by the average intensity of all spots not containing the modifcation. The graph shows the top ten most reactive histone modifications based on the specificity factor values. G9a is a known histone methyltransferse associated with the specific mono- and di-methylation of lysine $9 \mathrm{cn}$ Hstone H3. The data shown here confrm the association of G9a with its preferred histone modifications. 
Annotations for each site

\begin{tabular}{l|l|l|l|l|l|l|l|l|l|l|l|l|l|l|l|l|l|l|l|l|l|l|l|l|l|}
0 & 0 & 0 & 0 & 0 & 0 & 0 & 0 & 0 & 0 & 0 & 0 & 0 & 0 & 0 & 0 & 0 & 0 & 0 & 0 & 0 & 0 & 0 & 0 & 0 & 0
\end{tabular} O A1 A 2 A3 A 4 A 5 A 6 A 7 A 8 A 9 A 10A 11A 12A 13A 14A 15A 16A 17A 18A 19A 20A 21A 22A 23 A 240 - B1 B 2 B 3 B 4 B 5 B 6 B 7 B 8 B 9 B10 B11 B12 B13 B14 B15 B16 B17 B18 B19 B20 B21 B22 B23 B24 O

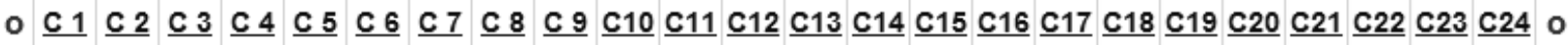

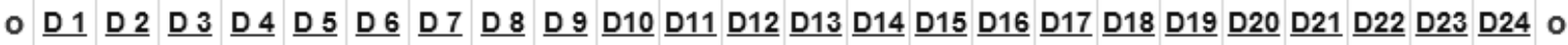
- E1 E 2 E3 E 4 E 5 E6 E 7 E 8 E9 E10 E11 E12 E13 E14 E15 E16 E17 E18 E19 E20 E21 E22 E23 E24 O - F1 F 2 F 3 F 4 F 5 F 6 F 7 F 8 F 9 F 10F 11F 12F 13F 14F 15F 16F 17F 18F 19F 20F 21F 22F 23F 240 - G1 G 2 G 3 G 4 G5 G6 G 7 G 8 G 9 G10 G11 G12 G13 G14 G15 G16 G17 G18 G19 G20 G21 G22 G23 G24 O ○ $\underline{H 1} \underline{H 2} \underline{H 3} \underline{H} 4 \underline{H 5} \underline{H 6} \underline{H 7} \underline{H} 8 \underline{H ~} \underline{H 10} \underline{H 11} \underline{H 12} \underline{H 13} \underline{H 14} \underline{H 15} \underline{H 16} \underline{H 17} \underline{H 18}$ H19 H20 H21 H22 H23 H24 O

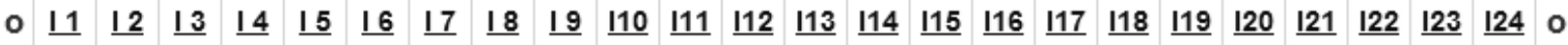
$\circ \underline{\mathrm{J} 1} \underline{\mathrm{J} 2} \underline{\mathrm{J} 3} \underline{\mathrm{J} 4} \underline{\mathrm{J} 5} \underline{\mathrm{J} 6} \underline{\mathrm{J} 7} \underline{\mathrm{J} 8} \mathbf{\mathrm { J } 9} \underline{\mathrm{J} 10} \underline{\mathrm{J} 11} \underline{\mathrm{J} 12} \underline{\mathrm{J} 13} \underline{\mathrm{J} 14} \underline{\mathrm{J} 15} \underline{\mathrm{J} 16} \underline{\mathrm{J} 17} \underline{\mathrm{J} 18} \underline{\mathrm{J} 19} \mathrm{J20} \underline{\mathrm{J} 21} \underline{\mathrm{J} 22} \underline{\mathrm{J} 23} \underline{\mathrm{J} 24} \mathrm{\circ}$ - $\underline{\text { K1 }} \underline{\mathrm{K} 2} \underline{\mathrm{K} 3} \underline{\mathrm{K} 4} \underline{\mathrm{K} 5} \underline{\mathrm{K} 6} \underline{\mathrm{K} 7} \underline{\mathrm{K} 8} \underline{\mathrm{K} 9} \underline{\mathrm{K} 10} \underline{\mathrm{K} 11} \underline{\mathrm{K} 12} \underline{\mathrm{K} 13} \underline{\mathrm{K} 14} \underline{\mathrm{K} 15} \underline{\mathrm{K} 16} \underline{\mathrm{K} 17} \underline{\mathrm{K} 18} \underline{\mathrm{K} 19} \underline{\mathrm{K} 20} \underline{\mathrm{K} 21} \underline{\mathrm{K} 22} \underline{\mathrm{K} 23} \underline{\mathrm{K} 24} \mathrm{O}$

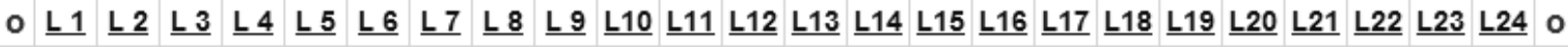
- M 1 M 2 M 3 M 4 M 5 M 6 M 7 M 8 M 9 M10M11M12 M13 M14 M15 M16M17 M18 M19M20M21 M22 M23 M24 O

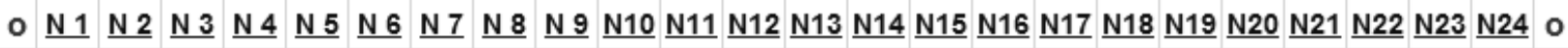

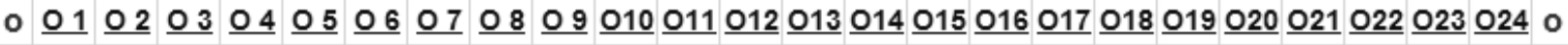

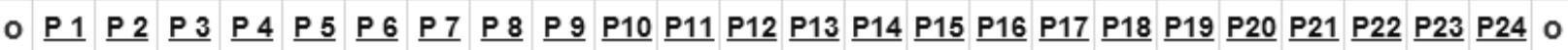
\begin{tabular}{l|l|l|l|l|l|l|l|l|l|l|l|l|l|l|l|l|l|l|l|l|l|l|l|l|l|}
0 & 0 & 0 & 0 & 0 & 0 & 0 & 0 & 0 & 0 & 0 & 0 & 0 & 0 & 0 & 0 & 0 & 0 & 0 & 0 & 0 & 0 & 0 & 0 & 0 & 0
\end{tabular}

Active Motif MODified ${ }^{\mathrm{TM}}$ Histone Peptide Array* Catalog Nos. 13001 \& 13005 ${ }^{*}$ CelluSpots ${ }^{\mathrm{TM}}$ arrays are manufactured under license by INTAVIS Bioanalytical Instruments AG

\begin{tabular}{|c|c|c|c|c|c|c|c|}
\hline Location & Peptide sequence & Name & Modl & Mod2 & Mod 3 & Mod 4 & N-terminus \\
\hline A 1 & A R T K Q T A R K S T G G K A P R K Q & H3 1-19 & unmod & & & & free \\
\hline A 2 & A Rme2s T K Q T A R K S T G G K A P R K Q & H3 1-19 & $\mathrm{R} 2 \mathrm{me} 2 \mathrm{~s}$ & & & & free \\
\hline A 3 & A Rme2a T K Q T A R K S T G G K A P R K Q & H3 1-19 & R2me2a & & & & free \\
\hline A 4 & A Cit TKQTAR KSTGGKAPRKQ & H3 1-19 & R2Citr & & & & free \\
\hline A 5 & A R pT K Q T A R K S T G G K A P R K Q & H3 1-19 & T3P & & & & free \\
\hline A 6 & A R T Kme1 Q T A R K S T G G K A P R K Q & H3 1-19 & K4me1 & & & & free \\
\hline A 7 & A R T Kme2 Q T A R K S T G G K A P R K Q & H3 1-19 & K4me2 & & & & free \\
\hline A 8 & A R T Kme3 Q T A R K S T G G K A P R K Q & H3 1-19 & K4me3 & & & & free \\
\hline A 9 & A R T Kac Q T A R K S T G G K A P R K Q & H3 1-19 & K4ac & & & & free \\
\hline A10 & A R T K Q T A Rme2s K S T G G K A P R K Q & H3 1-19 & R8me2s & & & & free \\
\hline A11 & A R T K Q T A Rme2a K S T G G K A P R K Q & H3 1-19 & R8me2a & & & & free \\
\hline A12 & A R T K Q T A Cit K S T G G K A P R K Q & H3 1-19 & R8Citr & & & & free \\
\hline A13 & A R T K Q T A R Kme1 S T G G K A P R K Q & H3 1-19 & K9me1 & & & & free \\
\hline A14 & A R T K Q T A R Kme2 S T G G K A P R K Q & H3 1-19 & K9me2 & & & & free \\
\hline A15 & A R T K Q T A R Kme3 S T G G K A P R K Q & H3 1-19 & K9me3 & & & & free \\
\hline A16 & A R T K Q T A R Kac S T G G K A P R K Q & H3 1-19 & K9ac & & & & free \\
\hline A17 & A R T K Q T A R K pS T G G K A P R K Q & H3 1-19 & S10P & & & & free \\
\hline A18 & A R T K Q T A R K S pT G G K A P R K Q & H3 1-19 & T11P & & & & free \\
\hline A19 & A R T K Q T AR KS T G G Kac A P R K Q & H3 1-19 & K14ac & & & & free \\
\hline
\end{tabular}




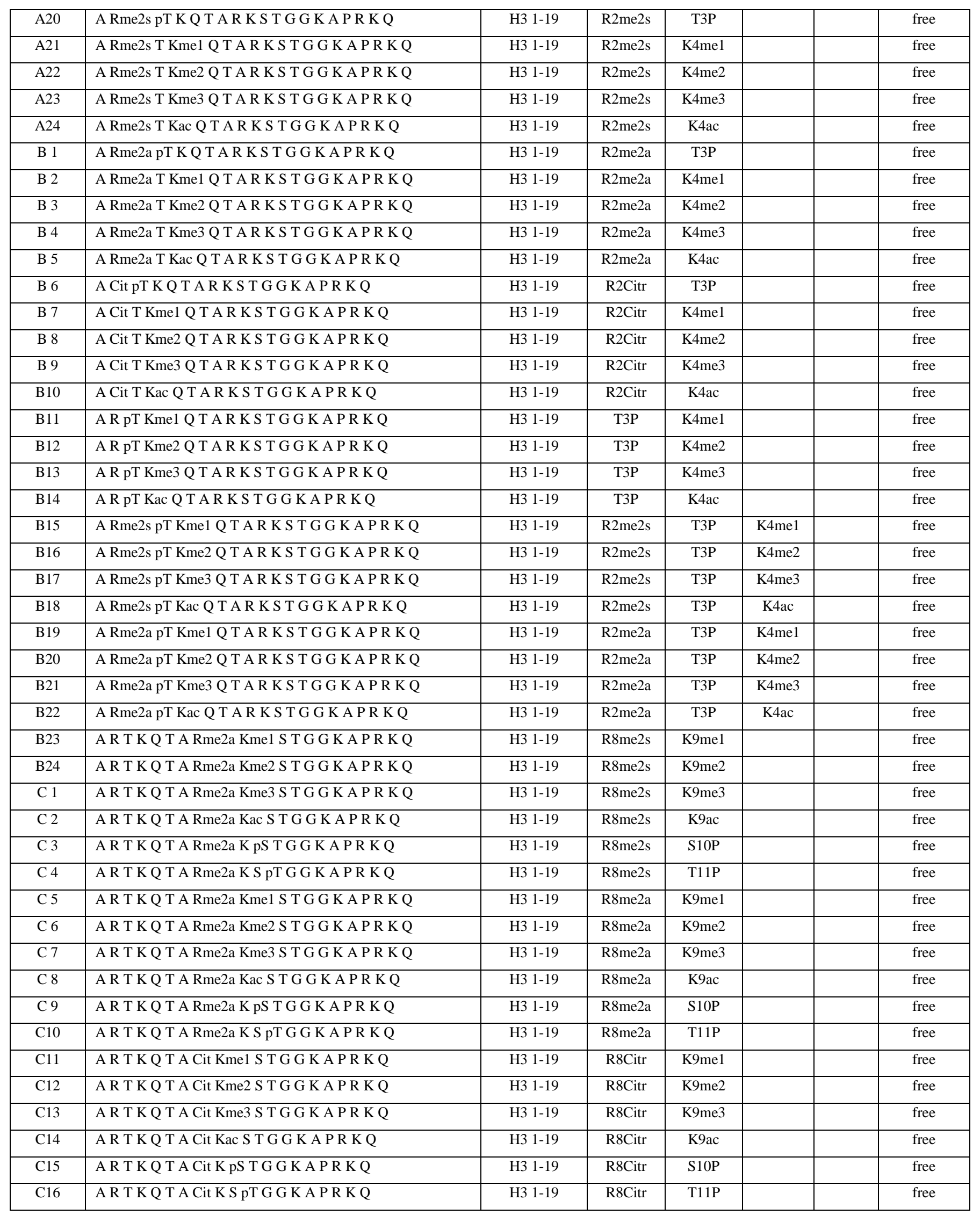




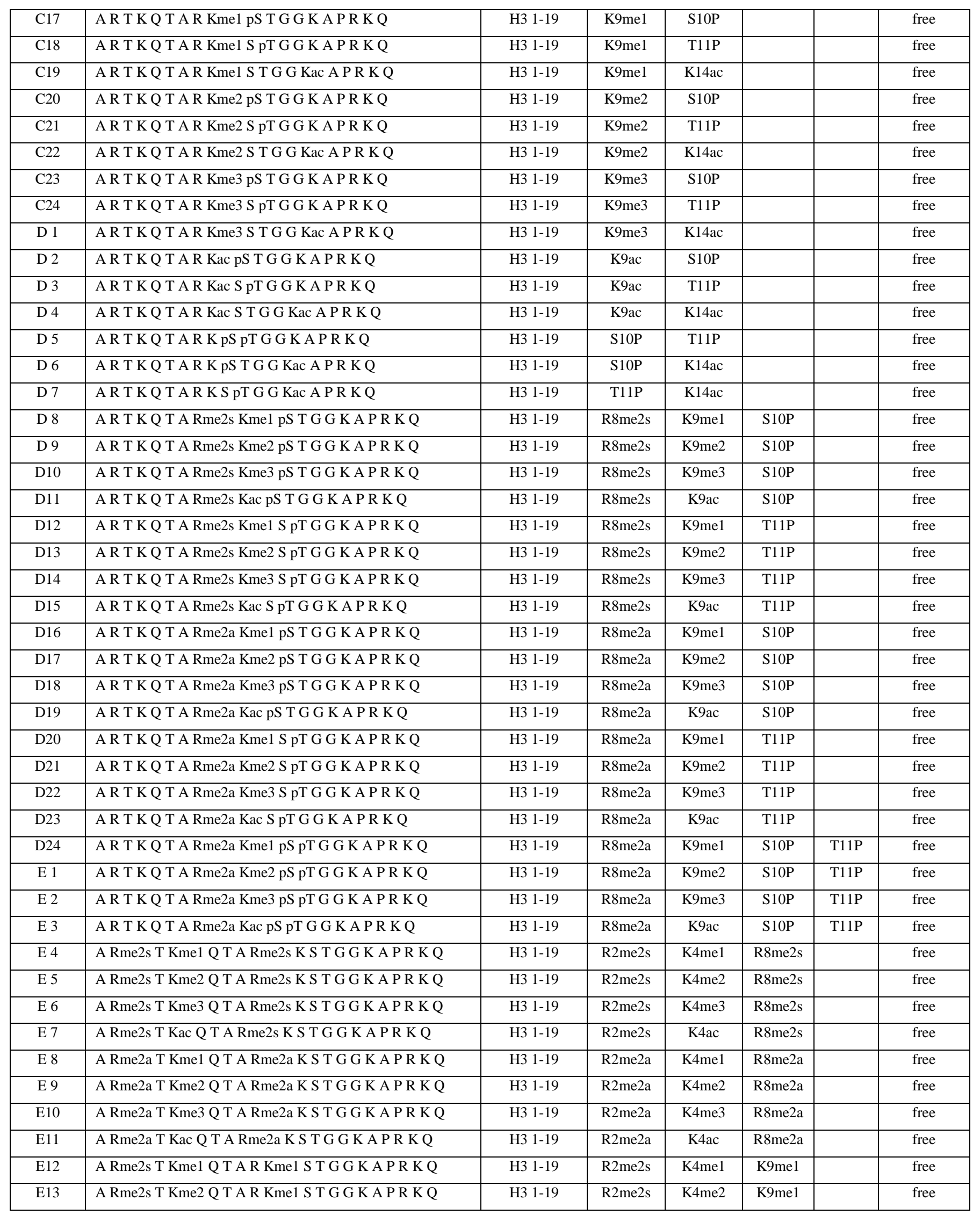




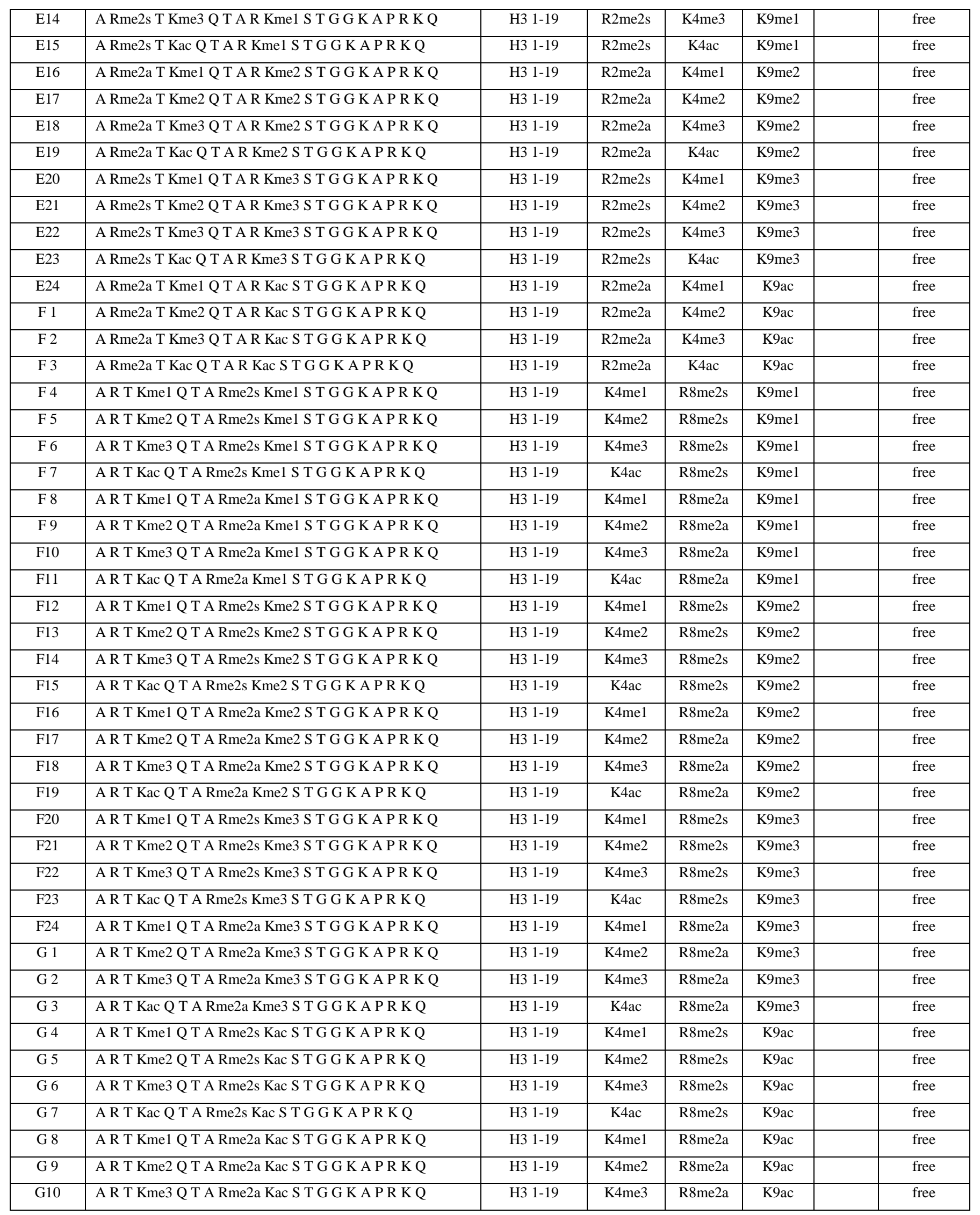




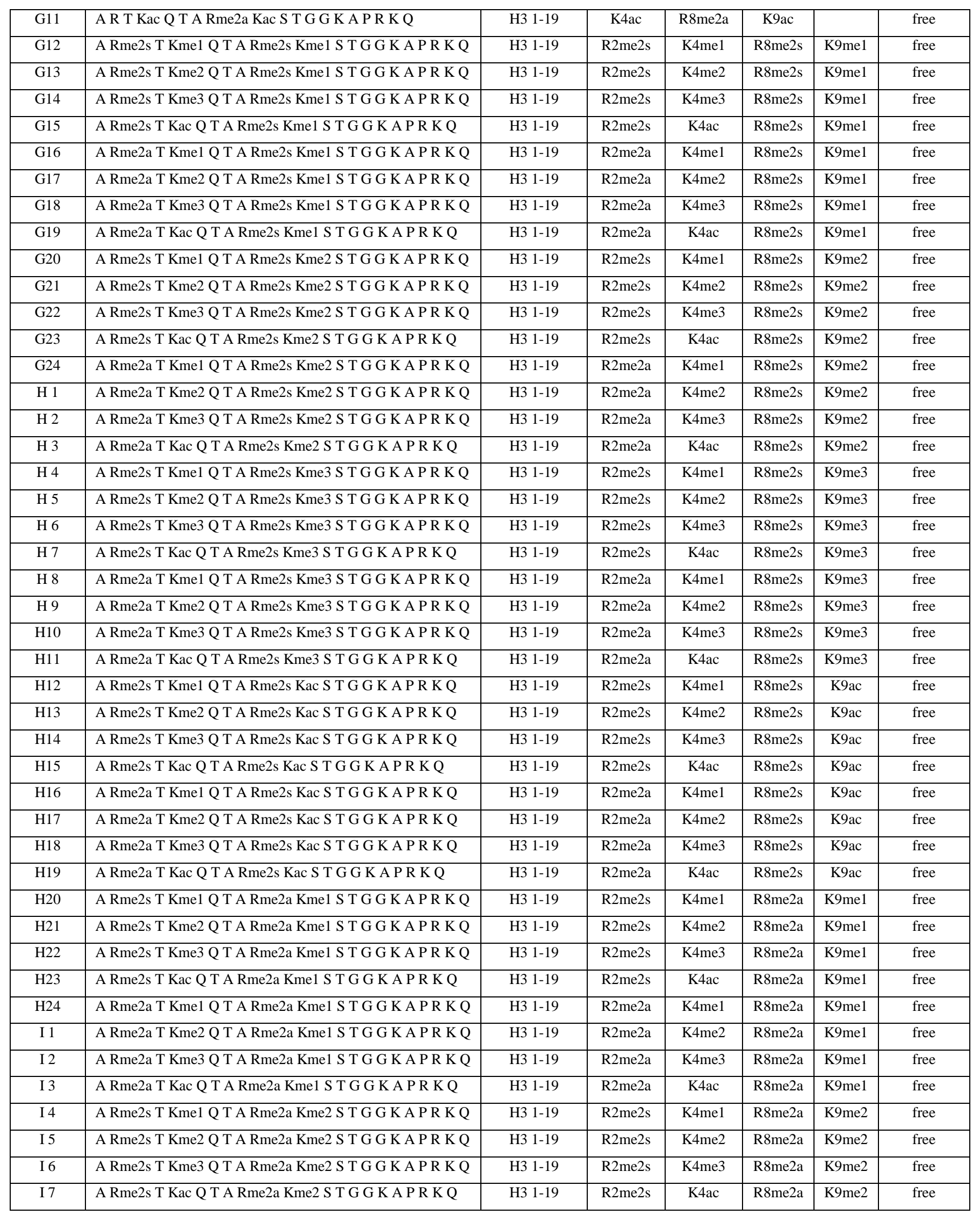




\begin{tabular}{|c|c|c|c|c|c|c|c|}
\hline I 8 & A Rme2a T Kme1 Q T A Rme2a Kme2 S T G G K A P R K Q & H3 1-19 & R2me2a & K4me1 & R8me2a & K9me2 & free \\
\hline I 9 & A Rme2a T Kme2 Q T A Rme2a Kme2 S T G G K A P R K Q & H3 1-19 & $\mathrm{R} 2 \mathrm{me} 2 \mathrm{a}$ & K4me2 & R8me2a & K9me2 & free \\
\hline $\mathrm{I} 10$ & A Rme2a T Kme3 Q T A Rme2a Kme2 S T G G K A P R K Q & H3 1-19 & R2me2a & K4me3 & R8me2a & K9me2 & free \\
\hline I11 & A Rme2a T Kac Q T A Rme2a Kme2 S T G G K A P R K Q & H3 1-19 & R2me2a & K4ac & R8me2a & K9me2 & free \\
\hline $\mathrm{I} 12$ & A Rme2s T Kme1 Q T A Rme2a Kme3 S T G G K A P R K Q & H3 1-19 & $\mathrm{R} 2 \mathrm{me} 2 \mathrm{~s}$ & K4me1 & R8me2a & K9me3 & free \\
\hline I13 & A Rme2s T Kme2 Q T A Rme2a Kme3 S T G G K A P R K Q & H3 1-19 & $\mathrm{R} 2 \mathrm{me} 2 \mathrm{~s}$ & K4me2 & R8me2a & K9me3 & free \\
\hline $\mathrm{I} 14$ & A Rme2s T Kme3 Q T A Rme2a Kme3 S T G G K A P R K Q & H3 1-19 & R2me2s & K4me3 & R8me2a & K9me3 & free \\
\hline I15 & A Rme2s T Kac Q T A Rme2a Kme3 S T G G K A P R K Q & H3 1-19 & R2me2s & K4ac & R8me2a & K9me3 & free \\
\hline I16 & A Rme2a T Kme1 Q T A Rme2a Kme3 S T G G K A P R K Q & H3 1-19 & R2me2a & K4me1 & R8me2a & K9me3 & free \\
\hline $\mathrm{I} 17$ & A Rme2a T Kme2 Q T A Rme2a Kme3 S T G G K A P R K Q & H3 1-19 & R2me2a & K4me2 & R8me2a & K9me3 & free \\
\hline I18 & A Rme2a T Kme3 Q T A Rme2a Kme3 S T G G K A P R K Q & H3 1-19 & $\mathrm{R} 2 \mathrm{me} 2 \mathrm{a}$ & K4me3 & R8me2a & K9me3 & free \\
\hline $\mathrm{I} 19$ & A Rme2a T Kac Q T A Rme2a Kme3 S T G G K A P R K Q & H3 1-19 & R2me2a & K4ac & R8me2a & K9me3 & free \\
\hline $\mathrm{I} 20$ & A Rme2s T Kme1 Q T A Rme2a Kac S T G G K A P R K Q & H3 1-19 & $\mathrm{R} 2 \mathrm{me} 2 \mathrm{~s}$ & K4me1 & R8me2a & K9ac & free \\
\hline $\mathrm{I} 21$ & A Rme2s T Kme2 Q T A Rme2a Kac S T G G K A P R K Q & H3 1-19 & R2me2s & K4me2 & R8me2a & K9ac & free \\
\hline $\mathrm{I} 22$ & A Rme2s T Kme3 Q T A Rme2a Kac S T G G K A P R K Q & H3 1-19 & $\mathrm{R} 2 \mathrm{me} 2 \mathrm{~s}$ & K4me3 & R8me2a & K9ac & free \\
\hline $\mathrm{I} 23$ & A Rme2s T Kac Q T A Rme2a Kac S T G G K A P R K Q & H3 1-19 & R2me2s & K4ac & R8me2a & K9ac & free \\
\hline $\mathrm{I} 24$ & A Rme2a T Kme1 Q T A Rme2a Kac S T G G K A P R K Q & H3 1-19 & $\mathrm{R} 2 \mathrm{me} 2 \mathrm{a}$ & K4me1 & R8me2a & K9ac & free \\
\hline $\mathrm{J} 1$ & A Rme2a T Kme2 Q T A Rme2a Kac S T G G K A P R K Q & H3 1-19 & R2me2a & K4me2 & R8me2a & K9ac & free \\
\hline $\mathrm{J} 2$ & A Rme2a T Kme3 Q T A Rme2a Kac S T G G K A P R K Q & H3 1-19 & $\mathrm{R} 2 \mathrm{me} 2 \mathrm{a}$ & K4me3 & R8me2a & K9ac & free \\
\hline J 3 & A Rme2a T Kac Q T A Rme2a Kac S T G G K A P R K Q & H3 1-19 & R2me2a & K4ac & R8me2a & K9ac & free \\
\hline $\mathrm{J} 4$ & A R K S T G G K A P R K Q L A T K A A R & H3 7-26 & unmod & & & & acetylated \\
\hline J 5 & A R K S T G G Kac A P R K Q L A T K A A R & H3 7-26 & K14ac & & & & acetylated \\
\hline J 6 & A R K pS T G G Kac A P R K Q L A T K A A R & H3 7-26 & K14ac & S10P & & & acetylated \\
\hline J 7 & A R K S pT G G Kac A P R K Q L A T K A A R & H3 7-26 & K14ac & T11P & & & acetylated \\
\hline $\mathrm{J} 8$ & A R K S T G G K A P Rme2s K Q L A T K A A R & H3 7-26 & R17me2s & & & & acetylated \\
\hline J 9 & A R K S T G G K A P Rme2a K Q L A T K A A R & H3 7-26 & $\mathrm{R} 17 \mathrm{me} 2 \mathrm{a}$ & & & & acetylated \\
\hline $\mathrm{J} 10$ & A R K S T G G K A P Cit K Q L A T K A A R & H3 7-26 & R17Citr & & & & acetylated \\
\hline $\mathrm{J} 11$ & A R K S T G G K A P R Kac Q L A T K A A R & H3 7-26 & K18ac & & & & acetylated \\
\hline $\mathrm{J} 12$ & A R K S T G G Kac A P Rme2s K Q L A T K A A R & H3 7-26 & K14ac & R17me2s & & & acetylated \\
\hline J13 & A R K S T G G Kac A P Rme2a K Q L A T K A A R & H3 7-26 & K14ac & R17me2a & & & acetylated \\
\hline $\mathrm{J} 14$ & A R K S T G G Kac A P R Kac Q L A T K A A R & H3 7-26 & K14ac & K18ac & & & acetylated \\
\hline $\mathrm{J} 15$ & A R K S T G G K A P Rme2s Kac Q L A T K A A R & H3 7-26 & R17me2s & K18ac & & & acetylated \\
\hline J16 & A R K S T G G K A P Rme2a Kac Q L A T K A A R & H3 7-26 & R17me2a & K18ac & & & acetylated \\
\hline J17 & A R K S T G G K A P Cit Kac Q L A T K A A R & H3 7-26 & R17Citr & K18ac & & & acetylated \\
\hline J18 & A R K S T G G Kac A P Rme2s Kac Q L A T K A A R & H3 7-26 & K14ac & R17me2s & K18ac & & acetylated \\
\hline J19 & A R K S T G G Kac A P Rme2a Kac Q L A T K A A R & H3 7-26 & K14ac & R17me2a & K18ac & & acetylated \\
\hline $\mathrm{J} 20$ & P R K Q L A T K A A R K S A P A T G G & H3 16-35 & unmod & & & & acetylated \\
\hline $\mathbf{J} 21$ & P R K Q L A T K A A Rme2s K S A P A T G G & H3 16-35 & R26me2s & & & & acetylated \\
\hline $\mathrm{J} 22$ & P R K Q L A T K A A Rme2a K S A P A T G G & H3 16-35 & R26me2a & & & & acetylated \\
\hline $\mathrm{J} 23$ & P R K Q L A T K A A Cit K S A P A T G G & H3 16-35 & R26Citr & & & & acetylated \\
\hline $\mathrm{J} 24$ & P R K Q L A T K A A R Kme1 S A P A T G G & H3 16-35 & K27me1 & & & & acetylated \\
\hline K 1 & P R K Q L A T K A A R Kme2 S A P A T G G & H3 16-35 & K27me2 & & & & acetylated \\
\hline K 2 & P R K Q L A T K A A R Kme3 S A P A T G G & H3 16-35 & K27me3 & & & & acetylated \\
\hline K 3 & P R K Q L A T K A A R Kac S A P A T G G & H3 16-35 & K27ac & & & & acetylated \\
\hline $\mathrm{K} 4$ & P R K Q L A T K A A R K pS A P A T G G & H3 16-35 & $\mathrm{S} 28 \mathrm{P}$ & & & & $\overline{\text { acetylated }}$ \\
\hline
\end{tabular}




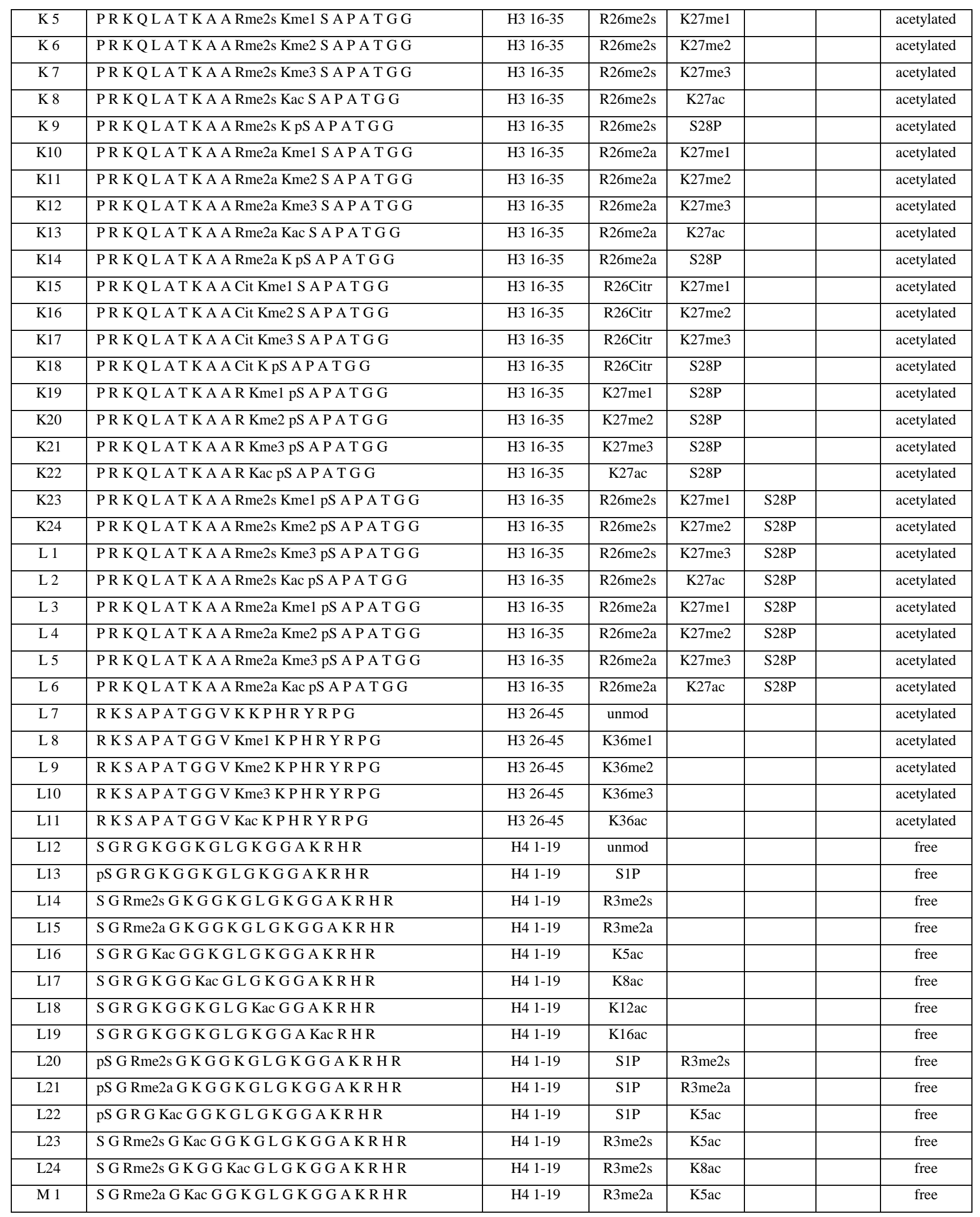




\begin{tabular}{|c|c|c|c|c|c|c|c|}
\hline M 2 & S G Rme2a G K G G Kac G L G K G G A K R H R & H4 1-19 & R3me2a & K8ac & & & free \\
\hline M 3 & S G R G Kac G G Kac G L G K G G A K R H R & H4 1-19 & K5ac & K8ac & & & free \\
\hline M4 & S G R G K G G Kac G L G Kac G G A K R H R & H4 1-19 & K8ac & K12ac & & & free \\
\hline M 5 & S G R G K G G Kac G L G K G G A Kac R H R & H4 1-19 & K8ac & K16ac & & & free \\
\hline M 6 & S G R G K G G K G L G Kac G G A Kac R H R & H4 1-19 & K12ac & K16ac & & & free \\
\hline M7 & pS G Rme2s G Kac G G K G L G K G G A K R H R & H4 1-19 & S1P & R3me2s & K5ac & & free \\
\hline M 8 & pS G Rme2a G Kac G G K G L G K G G A K R H R & H4 1-19 & S1P & R3me2a & K5ac & & free \\
\hline M9 & S G Rme2s G Kac G G Kac G L G K G G A K R H R & H4 1-19 & $\mathrm{R} 3 \mathrm{me} 2 \mathrm{~s}$ & K5ac & K8ac & & free \\
\hline M10 & S G Rme2a G Kac G G Kac G L G K G G A K R H R & H4 1-19 & R3me2a & K5ac & K8ac & & free \\
\hline M11 & S G R G Kac G G Kac G L G Kac G G A K R H R & H4 1-19 & K5ac & K8ac & $\mathrm{K} 12 \mathrm{ac}$ & & free \\
\hline M12 & S G R G K G G Kac G L G Kac G G A Kac R H R & H4 1-19 & K8ac & K12ac & K16ac & & free \\
\hline M13 & pS G Rme2s G Kac G G Kac G L G K G G A K R H R & H4 1-19 & S1P & R3me2s & K5ac & K8ac & free \\
\hline M14 & pS G Rme2a G Kac G G Kac G L G K G G A K R H R & H4 1-19 & S1P & R3me2a & K5ac & K8ac & free \\
\hline M15 & S G Rme2s G Kac G G Kac G L G Kac G G A K R H R & H4 1-19 & R3me2s & K5ac & K8ac & $\mathrm{K} 12 \mathrm{ac}$ & free \\
\hline M16 & S G Rme2a G Kac G G Kac G L G Kac G G A K R H R & H4 1-19 & R3me2a & K5ac & K8ac & K12ac & free \\
\hline M17 & S G R G Kac G G Kac G L G Kac G G A Kac R H R & H4 1-19 & K5ac & K8ac & $\mathrm{K} 12 \mathrm{ac}$ & K16ac & free \\
\hline M18 & G K G G A K R H R K V L R D N I Q G I T & H4 11-30 & unmod & & & & acetylated \\
\hline M19 & G Kac G G A K R H R K V L R D N I Q G I T & H4 11-30 & $\mathrm{K} 12 \mathrm{ac}$ & & & & acetylated \\
\hline M20 & G K G G A Kac R H R K V L R D N I Q G I T & H4 11-30 & K16ac & & & & acetylated \\
\hline M21 & G K G G A K Rme2s H R K V L R D N I Q G I T & H4 11-30 & R17me2s & & & & acetylated \\
\hline M22 & G K G G A K Rme2a H R K V L R D N I Q G I T & H4 11-30 & R17me2a & & & & acetylated \\
\hline M23 & G K G G A K R H Rme2s K V L R D N I Q G I T & H4 11-30 & R19me2s & & & & acetylated \\
\hline M24 & G K G G A K R H Rme2a K V L R D N I Q G I T & H4 11-30 & R19me2a & & & & acetylated \\
\hline $\mathrm{N} 1$ & G K G G A K R H R Kme1 V L R D N I Q G I T & H4 11-30 & K20me1 & & & & acetylated \\
\hline $\mathrm{N} 2$ & G K G G A K R H R Kme2 V L R D N I Q G I T & H4 11-30 & K20me2 & & & & acetylated \\
\hline N3 & G K G G A K R H R Kme3 V L R D N I Q G I T & H4 11-30 & K20me3 & & & & acetylated \\
\hline $\mathrm{N} 4$ & G K G G A K R H R Kac V L R D N I Q G I T & H4 11-30 & K20ac & & & & acetylated \\
\hline N 5 & G K G G A K R H R K V L Rme2a D N I Q G I T & H4 11-30 & R24me2a & & & & acetylated \\
\hline N 6 & G K G G A K R H R K V L Rme2s D N I Q G I T & H4 11-30 & R24me2s & & & & acetylated \\
\hline N 7 & G Kac G G A Kac R H R K V L R D N I Q G I T & H4 11-30 & K12ac & K16ac & & & acetylated \\
\hline N 8 & G K G G A Kac Rme2s H R K V L R D N I Q G I T & H4 11-30 & K16ac & R17me2s & & & acetylated \\
\hline N9 & G K G G A Kac Rme2a H R K V L R D N I Q G I T & H4 11-30 & K16ac & R17me2a & & & acetylated \\
\hline N10 & G K G G A Kac R H Rme2s K V L R D N I Q G I T & H4 11-30 & K16ac & R19me2s & & & acetylated \\
\hline N11 & G K G G A Kac R H Rme2a K V L R D N I Q G I T & H4 11-30 & K16ac & R19me2a & & & acetylated \\
\hline N12 & G K G G A Kac R H R Kme1 V L R D N I Q G I T & H4 11-30 & K16ac & K20me1 & & & acetylated \\
\hline N13 & G K G G A Kac R H R Kme2 V L R D N I Q G I T & H4 11-30 & K16ac & K20me2 & & & acetylated \\
\hline N14 & G K G G A Kac R H R Kme3 V L R D N I Q G I T & $\mathrm{H} 4$ 11-30 & K16ac & K20me3 & & & acetylated \\
\hline N15 & G K G G A Kac R H R Kac V L R D N I Q G I T & H4 11-30 & K16ac & K20ac & & & acetylated \\
\hline N16 & G Kac G G A Kac R H R Kme1 V L R D N I Q G I T & $\mathrm{H} 4$ 11-30 & $\mathrm{K} 12 \mathrm{ac}$ & K16ac & K20me1 & & acetylated \\
\hline N17 & G Kac G G A Kac R H R Kme2 V L R D N I Q G I T & H4 11-30 & K12ac & K16ac & K20me2 & & acetylated \\
\hline N18 & G Kac G G A Kac R H R Kme3 V L R D N I Q G I T & H4 11-30 & $\mathrm{K} 12 \mathrm{ac}$ & K16ac & K20me3 & & acetylated \\
\hline N19 & G Kac G G A Kac R H R Kac V L R D N I Q G I T & H4 11-30 & K12ac & K16ac & K20ac & & acetylated \\
\hline $\mathrm{N} 20$ & G K G G A K R H Rme2a Kme1 V L R D N I Q G I T & H4 11-30 & R19me2a & K20me1 & & & acetylated \\
\hline $\mathrm{N} 21$ & G K G G A K R H Rme2a Kme2 V L R D N I Q G I T & H4 11-30 & R19me2a & K20me2 & & & acetylated \\
\hline $\mathrm{N} 22$ & G K G G A K R H Rme2a Kme3 V L R D N I Q G I T & H4 11-30 & R19me2a & K20me3 & & & acetylated \\
\hline
\end{tabular}




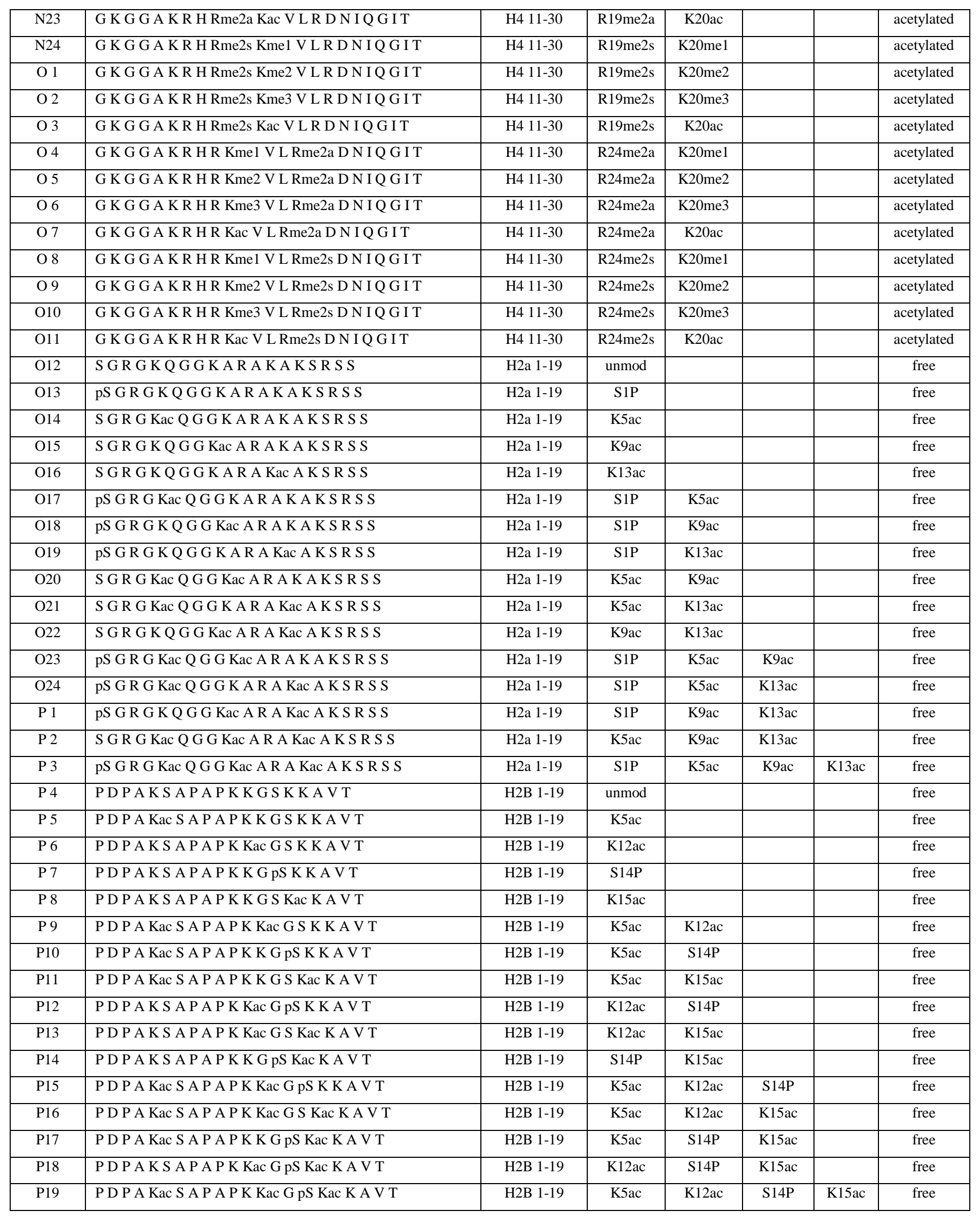




\begin{tabular}{|c|l|c|c|c|c|c|}
\hline P20 & Bio A A N W S H P Q F E K A A & $\begin{array}{c}\text { Biotin, } \\
\text { control } \\
\text { peptide }\end{array}$ & & biotinylated \\
\hline P21 & E Q K L I S E E D L A & c-myc tag & & & & \\
\hline P22 & HAc & neg. contol & & & & free \\
\hline P23 & K Kme1 Kme2 Kme3 Kac R Rme2s R Rme2a R Cit K Kme1 & background & & & acetylated \\
& Kac Kme3 R K & 01 & & acetylated \\
\hline P24 & R Rme2s K Kme1 Kac R Rme2a Kme2 K Kme3 R Kme1 & background \\
& Rme2s K Kac R K & & & & acetylated \\
\hline
\end{tabular}




\section{REFERENCES}

Aalfs, J. D., \& Kingston, R. E. (2000). Review: What does "chromatin remodeling" mean? Trends in Biochemical Sciences, 25, 548-555.

Allis, C. D., Richman, R., Gorovsky, M. a, Ziegler, Y. S., Touchstone, B., Bradley, W. a, \& Cook, R. G. (1986). hv1 is an evolutionarily conserved H2A variant that is preferentially associated with active genes. The Journal of Biological Chemistry, 261(4), 1941-1948.

Badenhorst, P., Voas, M., Rebay, I., \& Wu, C. (2002). Biological functions of the ISWI chromatin remodeling complex NURF. Genes and Development, 16, 3186-3198.

Baker, S. P., \& Grant, P. a. (2007). The SAGA continues: expanding the cellular role of a transcriptional co-activator complex. Oncogene, 26, 5329-5340.

Barker, N., Hurlstone, A., Musisi, H., Miles, A., Bienz, M., \& Clevers, H. (2001). The chromatin remodelling factor Brg-1 interacts with beta-catenin to promote target gene activation. The EMBO Journal, 20, 4935-4943.

Berger, S. L. (2007). The complex language of chromatin regulation during transcription. Nature, 447, 407-412.

Biterge, B., \& Schneider, R. (2014). Histone variants: Key players of chromatin. Cell and Tissue Research, 356, 457-466.

Blackburn, E. H., Greider, C. W., \& Szostak, J. W. (2006). Telomeres and telomerase: the path from maize, Tetrahymena and yeast to human cancer and aging. Nature Medicine, 12, $1133-1138$.

Bochar, D. a, Wang, L., Beniya, H., Kinev, A., Xue, Y., Lane, W. S., ... Shiekhattar, R. (2000). BRCA1 is associated with a human SWI/SNF-related complex: linking chromatin remodeling to breast cancer. Cell, 102, 257-265.

Breeden, L., \& Nasmyth, K. (1987). Cell cycle control of the yeast HO gene: cis- and transacting regulators. Cell, 48, 389-97.

Bright, L. J., Kambesis, N., Nelson, S. B., Jeong, B., \& Turkewitz, A. P. (2010). Comprehensive analysis reveals dynamic and evolutionary plasticity of Rab GTPases and membrane traffic in Tetrahymena thermophila. PLoS Genetics, 6, 1-18.

Brownell, J. E., Zhou, J., Ranalli, T., Kobayashi, R., Edmondson, D. G., Roth, S. Y., \& Allis, C. D. (1996). Tetrahymena histone acetyltransferase: a homologue to yeast Gcn5p linking histone acetylation to gene activation. Cell, 84, 843-851. 
Bruns, P. J., \& Cassidy-Hanley, D. (1999). Biolistic transformation of macro- and micronuclei. Methods in Molecular Biology, 62, 501-512.

Burnette, N. W. (1981). "Western Blotting": Electrophoretic Transfer of Proteins from Sodium Dodecyl Sulfate-Polyacrylamide Gels to Unmodified Nitrocellulose and Radiographic Detection with Antibody and Radioiodinated Protein A. Analytical Biochemistry, 112, 195203.

Cairns, B. R., Lorch, Y., Li, Y., Zhang, M., Lacomis, L., Erdjument-Bromage, H., ... Kornberg, R. D. (1996). RSC, an essential, abundant chromatin-remodeling complex. Cell, 87, 12491260.

Cassidy-Hanley, D., Bowen, J., Lee, J. H., Cole, E., Verplank, L. A., Gaertip, J., ... Bruns, P. J. (1997). Germline and Somatic Transformation of Mating Tetrahymena thermophila by Particle Bombardment. Genetics Society of America, 146, 135-147.

Cech, T. R. (1990). Self-splicing and enzymatic activity of an intervening sequence RNA from Tetrahymena. Bioscience Reports, 24, 362-385.

Cervantes, M. D., Hamilton, E. P., Xiong, J., Lawson, M. J., Yuan, D., Hadjithomas, M., ... Orias, E. (2013). Selecting One of Several Mating Types through Gene Segment Joining and Deletion in Tetrahymena thermophila. PLoS Biology, 11(3), e1001518.

Chalker, D. L. (2008). Dynamic nuclear reorganization during genome remodeling of Tetrahymena. Biochimica et Biophysica Acta, 1783(11), 2130-6.

Chalker, D. L., Terza, A. La, Wilson, A., Kroenke, C. D., Yao, M., \& Terza, A. L. A. (1999). Flanking Regulatory Sequences of the Tetrahymena R Deletion Element Determine the Boundaries of DNA Rearrangement Flanking Regulatory Sequences of the Tetrahymena R Deletion Element Determine the Boundaries of DNA Rearrangement. Molecular and Cellular Biology, 19(8), 5631-5641.

Chalker, D. L., \& Yao, M. C. (2001). Nongenic, bidirectional transcription precedes and may promote developmental DNA deletion in Tetrahymena thermophila. Genes and Development, 15, 1287-1298.

Collins, K. (2012). Methods in Cell Biology Volume 109 - Tetrahymena thermophila. In Academic Press (p. 13).

Collins, R. E., Northrop, J. P., Horton, J. R., Lee, D. Y., Zhang, X., Stallcup, M. R., \& Cheng, X. (2008). The ankyrin repeats of G9a and GLP histone methyltransferases are mono- and dimethyllysine binding modules. Nature Structural \& Molecular Biology, 15, 245-250.

Coyne, R. S., Nikiforov, M. a, Smothers, J. F., Allis, C. D., \& Yao, M. C. (1999). Parental expression of the chromodomain protein Pdd1p is required for completion of programmed DNA elimination and nuclear differentiation. Molecular Cell, 4(5), 865-872. 
Cuatrecasas, P. (1970). Protein Purification by Affinity Chromatography. Journal of Biological Sciences, 245, 3050-3065.

Dingwall, a K., Beek, S. J., McCallum, C. M., Tamkun, J. W., Kalpana, G. V, Goff, S. P., \& Scott, M. P. (1995). The Drosophila snr1 and brm proteins are related to yeast SWI/SNF proteins and are components of a large protein complex. Molecular Biology of the Cell, 6, 777-791.

Eisen, J. a, Sweder, K. S., \& Hanawalt, P. C. (1995). Evolution of the SNF2 family of proteins: subfamilies with distinct sequences and functions. Nucleic Acids Research, 23, 2715-2723.

Erdel, F., Schubert, T., Marth, C., Längst, G., \& Rippe, K. (2010). Human ISWI chromatinremodeling complexes sample nucleosomes via transient binding reactions and become immobilized at active sites. Proceedings of the National Academy of Sciences of the United States of America, 107, 19873-19878.

Fenn, J. B., Mann, M., Meng, C. K., Wong, S. F., Craig, M., Meng, C. K. A. I., ... Whitehouse, C. M. (2013). Electrospray Ionization of Large for Mass Spectrometry Biomolecules. American Association for the Advancement of Science, 246(4926), 64-71.

Filippakopoulos, P., Picaud, S., Mangos, M., Keates, T., Lambert, J. P., Barsyte-Lovejoy, D., ... Knapp, S. (2012). Histone recognition and large-scale structural analysis of the human bromodomain family. Cell, 149, 214-231.

Fillingham, J. S., Garg, J., Tsao, N., Vythilingum, N., Nishikawa, T., \& Pearlman, R. E. (2006). Molecular genetic analysis of an SNF2/brahma-related gene in Tetrahymena thermophila suggests roles in growth and nuclear development. Eukaryotic Cell, 5, 1347-1359.

Fischle, W., Wang, Y., \& Allis, C. D. (2003). Histone and chromatin cross-talk. Current Opinion in Cell Biology, 15, 172-183.

Gaertig, J., Gu, L., Hai, B., \& Gorovsky, M. a. (1994). High frequency vector-mediated transformation and gene replacement in Tetrahymena. Nucleic Acids Research, 22, 53915398.

Garg, J., Lambert, J.-P., Karsou, A., Marquez, S., Nabeel-Shah, S., Bertucci, V., ... Fillingham, J. S. (2013a). Conserved Asf1-importin $\beta$ physical interaction in growth and sexual development in the ciliate Tetrahymena thermophila. Journal of Proteomics, 94, 311-326.

Garg, J., Lambert, J.-P., Karsou, A., Marquez, S., Nabeel-Shah, S., Bertucci, V., ... Fillingham, J. S. (2013b). Conserved Asf1-importin $\beta$ physical interaction in growth and sexual development in the ciliate Tetrahymena thermophila. Journal of Proteomics, 94C, 311-326.

Holliday, R. (2006). Epigenetics: A historical overview. Epigenetics, 1, 76-80. 
Kamakaka, R., \& Hiller, N. (2005). Histone variants: deviants? Genes and Development, 19, 295-310.

Kataoka, K., Schoeberl, U. E., \& Mochizuki, K. (2010). Modules for C-terminal epitope tagging of Tetrahymena genes. Journal of Microbiological Methods, 82, 342-346.

Kent, N. a., Karabetsou, N., Politis, P. K., \& Mellor, J. (2001). In vivo chromatin remodeling by yeast ISWI homologs Isw1p and Isw2p. Genes and Development, 15, 619-626.

Knappik, A., \& Pluckthun, A. (1994). An Improved Affinity Tag Based on the FLAG Peptide for the Detection and Purification of Recombinant Antibody Fragments. BioTechniques, 17, 754-761.

Korochkin, L. I. (2006). What is epigenetics. Genetika, 42, 1156-1164.

Krogan, N. J., Keogh, M.-C., Datta, N., Sawa, C., Ryan, O. W., Ding, H., ... Greenblatt, J. F. (2003). A Snf2 family ATPase complex required for recruitment of the histone H2A variant Htz1. Molecular Cell, 12, 1565-1576.

Liu, X., Li, B., \& GorovskyMA. (1996). Essential and nonessential histone H2A variants in Tetrahymena thermophila. Molecular and Cellular Biology, 16, 4305-4311.

Liu, Y., Taverna, S. D., Muratore, T. L., Shabanowitz, J., Hunt, D. F., \& Allis, C. D. (2007). RNAi-dependent H3K27 methylation is required for heterochromatin formation and DNA elimination in Tetrahymena. Genes \& Development, 21(12), 1530-45. doi:10.1101/gad.1544207

Logie, C., \& Peterson, C. L. (1999). Purification and Biochemical Properties of Yeast SWI/SNF Complex. Methods in Enzymology, 304, 726-741.

Luger, K., Mader, A. W., Richmond, R. K., Sargent, D. F., \& Richmond, T. J. (1997). Crystal structure of the nucleosome resolution core particle at 2.8 A. Nature, 389, 251-260.

Madireddi, M. T., Coyne, R. S., Smothers, J. F., Mickey, K. M., Yao, M. C., \& Allis, C. D. (1996). Pdd1p, a novel chromodomain-containing protein, links heterochromatin assembly and DNA elimination in Tetrahymena. Cell, 87, 75-84.

Malone, C. D., Anderson, A. M., Motl, J. A., Rexer, C. H., \& Chalker, D. L. (2005). Germ Line Transcripts Are Processed by a Dicer-Like Protein That Is Essential for Developmentally Programmed Genome Rearrangements of Tetrahymena thermophila. Molecular and Cellular Biology, 25, 9151-9164.

Martindale, D. W., Allis, C. D., \& Bruns, P. J. (1982). Conjugation in Tetrahymena thermophila. A Temporal Analysis of Cytological Stages. Experimental Cell Research, 140, 227-236. 
Martindale, D. W., Allis, C. D., \& Bruns, P. J. (1985). RNA and protein Synthesis During Meiotic Prophase in Tetrahymena thermophila. Protozoology, 32, 345-350.

Merriam, E. V, \& Bruns, P. J. (1988). Phenotypic assortment in Tetrahymena thermophila: assortment kinetics of antibiotic-resistance markers, tsA, death, and the highly amplified rDNA locus. Genetics, 120, 389-395.

Mochizuki, K. (2012). Developmentally programmed, RNA-directed genome rearrangement in Tetrahymena. Development Growth and Differentiation, 54, 108-119.

Mochizuki, K., Fine, N. a, Fujisawa, T., \& Gorovsky, M. a. (2002). Analysis of a piwi-related gene implicates small RNAs in genome rearrangement in Tetrahymena. Cell, 110, 689-699.

Mochizuki, K., \& Gorovsky, M. a. (2004a). RNA polymerase II localizes in Tetrahymena thermophila meiotic micronuclei when micronuclear transcription associated with genome rearrangement occurs. Eukaryotic Cell, 3, 1233-1240.

Mochizuki, K., \& Gorovsky, M. a. (2004b). Small RNAs in genome rearrangement in Tetrahymena. Current Opinion in Genetics and Development, 14, 181-187.

Muchardt, C., Reyes, J. C., Bourachot, B., Leguoy, E., \& Yaniv, M. (1996). The hbrm and BRG1 proteins, components of the human SNF/SWI complex, are phosphorylated and excluded from the condensed chromosomes during mitosis. The EMBO Journal, 15, 3394-3402.

Nash, E. a., Nisbet, R. E. R., Barbrook, A. C., \& Howe, C. J. (2008). Dinoflagellates: a mitochondrial genome all at sea. Trends in Genetics, 24, 328-335.

Neigeborn, L., \& Carlson, M. (1984). Genes Affecting The Regulation Of SUC2 Gene Expression By Glucose Repression In Saccharomyces cerevisiae. Genetics Society of America, 108, 845-858.

Nguyen, V. Q., Ranjan, A., Stengel, F., Wei, D., Aebersold, R., Wu, C., \& Leschziner, A. E. (2013). Molecular architecture of the ATP-dependent chromatin-remodeling complex SWR1. Cell, 154, 1220-1231.

Nielsen, A. L., Sanchez, C., Ichinose, H., Cerviño, M., Lerouge, T., Chambon, P., \& Losson, R. (2002). Selective interaction between the chromatin-remodeling factor BRG1 and the heterochromatin-associated protein HP1alpha. The EMBO Journal, 21, 5797-5806.

Orias, E., Cervantes, M. D., \& Hamilton, E. P. (2011). Tetrahymena thermophila, a unicellular eukaryote with separate germline and somatic genomes. Research in Microbiology, 162, 578-586.

Otsuki, T., Furukawa, Y., Ikeda, K., Endo, H., Yamashita, T., Shinohara, A., ... Liu, J. M. (2001). Fanconi anemia protein, FANCA, associates with BRG1, a component of the human SWI/SNF complex. Human Molecular Genetics, 10, 2651-2660. 
Owen-hughes, T. (2003). Pathways for remodelling chromatin. Colworth Medal Lecture, 31, 893-905.

Pal, S., Vishwanath, S. N., Tempst, P., Sif, S., \& Erdjument-bromage, H. (2004). Human SWI/SNF-Associated PRMT5 Methylates Histone H3 Arginine 8 and Negatively Regulates Expression of ST7 and NM23 Tumor Suppressor Genes. Molecular and Cellular Biology, 24, 9630-9645.

Peterson, C. L., Dingwall, A., \& Scott, M. P. (1994). Five SWI/SNF gene products are components of a large multisubunit complex required for transcriptional enhancement. Proceedings of the National Academy of Sciences of the United States of America, 91, 2905-2908.

Reisman, D., Glaros, S., \& Thompson, E. a. (2009). Review: The SWI/SNF complex and cancer. Oncogene, 28, 1653-1668.

Rexer, C. H., \& Chalker, D. L. (2007). Lialp, a novel protein required during nuclear differentiation for genome-wide DNA rearrangements in Tetrahymena thermophila. Eukaryotic Cell, 6, 1320-1329.

Roberts, C. W. M., \& Orkin, S. H. (2004). Review: The SWI/SNF complex--chromatin and cancer. Nature Reviews. Cancer, 4, 133-142.

Roy, S., \& Morse, D. (2013). Transcription and Maturation of mRNA in Dinoflagellates. Microorganisms, 1, 71-99.

Schwope, R. M., \& Chalker, D. L. (2014). Mutations in Pdd1 reveal distinct requirements for its chromodomain and chromoshadow domain in directing histone methylation and heterochromatin elimination. Eukaryotic Cell, 13, 190-201.

Sigh, J., Lindenstrøm, T., \& Buchmann, K. (2004). Expression of pro-inflammatory cytokines in rainbow trout (Oncorhynchus mykiss) during an infection with Ichthyophthirius multifiliis. Fish \& Shellfish Immunology, 17, 75-86.

Skarra, D. V, Goudreault, M., Choi, H., Mullin, M., Nesvizhskii, A. I., Gingras, A.-C., \& Honkanen, R. E. (2011). Label-free quantitative proteomics and SAINT analysis enable interactome mapping for the human Ser/Thr protein phosphatase 5. Proteomics, 11, 15081516.

Slotkin, R. K., \& Martienssen, R. (2007). Transposable elements and the epigenetic regulation of the genome. Nature Reviews. Genetics, 8(4), 272-285.

Smothers, J. F., Mizzen, C. A., Tubbert, M. M., Cook, R. G., \& Allis, C. D. (1997). Pdd1p associates with germline-restricted chromatin and a second novel anlagen-enriched protein in developmentally programmed DNA elimination structures. Development (Cambridge, England), 124, 4537-4545. 
Stargell, L. a., Bowen, J., Dadd, C. a., Dedon, P. C., Davis, M., Cook, R. G., .. Gorovsky, M. a. (1993). Temporal and spatial association of his tone H2A variant hv1 with transcriptionally competent chromatin during nuclear development in Tetrahymena thermophila. Genes and Development, 7, 2641-2651.

Strahl, B. D., \& Allis, C. D. (2000). The language of covalent histone modifications. Nature, 403, 41-45.

Tamkun, J. W., Deuring, R., Scott, M. P., Kissinger, M., Pattatucci, a M., Kaufman, T. C., \& Kennison, J. a. (1992). brahma: a regulator of Drosophila homeotic genes structurally related to the yeast transcriptional activator SNF2/SWI2. Cell, 68, 561-572.

Tang, L., Nogales, E., \& Ciferri, C. (2010). Review: Structure and function of SWI/SNF chromatin remodeling complexes and mechanistic implications for transcription. Progress in Biophysics and Molecular Biology, 102, 122-128.

Taverna, S. D., Coyne, R. S., \& Allis, C. D. (2002). Methylation of Histone H3 at Lysine 9 Targets Programmed DNA Elimination in Tetrahymena. Cell, 110, 701-711.

Taverna, S. D., Ueberheide, B. M., Liu, Y., Tackett, A. J., Diaz, R. L., Shabanowitz, J., ... Allis, C. D. (2007). Long-distance combinatorial linkage between methylation and acetylation on histone H3 N termini. Proceedings of the National Academy of Sciences of the United States of America, 104, 2086-2091.

Teo, G., Liu, G., Zhang, J., Nesvizhskii, A. I., Gingras, A. C., \& Choi, H. (2014). SAINTexpress: Improvements and additional features in Significance Analysis of INTeractome software. Journal of Proteomics, 100, 37-43.

Turner, B. M., Birley, a J., \& Lavender, J. (1992). Histone H4 isoforms acetylated at specific lysine residues define individual chromosomes and chromatin domains in Drosophila polytene nuclei. Cell, 69, 375-384.

Van, C., Williams, J. S., Kunkel, T. a., \& Peterson, C. L. (2015). Deposition of histone H2A.Z by the SWR-C remodeling enzyme prevents genome instability. DNA Repair, 25, 9-14.

Versteege, I., Sevenet, N., Lange, J., Rousseau-Merck, M.-F., Ambros, P., Handgretinger, R., ... Delattre, O. (1998). Truncating mutations of hSNF5/INI1 in aggressive paediatric cancer. Nature, 394, 203-206.

Vignali, M., Hassan, A. H., Neely, K. E., \& Workman, J. L. (2000). ATP-Dependent ChromatinRemodeling Complexes. Molecular and Cellular Biology, 20, 1899-1910.

Wenkert, D., \& Allis, C. D. (1984). Timing of the appearance of macronuclear-specific histone variant hv1 and gene expression in developing new macronuclei of Tetrahymena thermophila. The Journal of Cell Biology, 98, 2107-2117. 
Williams, N. E., \& Hontst, J. E. (1995). Isolation and Fractionation of the Tetrahymena Cytoskeleton and Oral Apparatus. Methods in Cell Biology, 47, 301-306.

Winston, F., \& Carlson, M. (1992). Yeast SNF/SWI transcriptional activators and the SPT/SIN chromatin connection. Trends in Genetics, 8, 387-391.

Wolfe, J. (1967). Structural Aspects of Amitosis: A Light and Electron Microscope Study of the Isolated Macronuclei of Parameium aurelia and Tetrahymena pyriformis. Chromosoma, 23, $59-79$.

Wong, A. K. C., Shanahan, F., Chen, Y., Lian, L., Ha, P., Hendricks, K., ... Lees, E. (2000). BRG1, a Component of the SWI-SNF Complex, Is Mutated in Multiple Human Tumor Cell Lines. Cancer Reseach, 60, 6171-6177.

Yu, G. L., Hasson, M., \& Blackburn, E. H. (1988). Circular ribosomal DNA plasmids transform Tetrahymena thermophila by homologous recombination with endogenous macronuclear ribosomal DNA. Proceedings of the National Academy of Sciences of the United States of America, 85(14), 5151-5155. 